\title{
SECOND-GENERATION EVALUATION of a CORRECTIONAL COMMUNITY \\ SUBSTANCE ABUSE PROGRAM
}

by

\section{CHANTAL MARTEEN LANGEVIN, B.A., M.A.}

\author{
A dissertation submitted to \\ the Faculty of Graduate Studies and Research \\ in partial fulfillment of \\ the requirements for the degree of \\ Doctor of Philosophy \\ Department of Psychology
Carleton University
Ottawa, Ontario \\ November 2004 \\ (C) copyright \\ 2004, Chantal Marteen Langevin
}




$\begin{array}{ll}\begin{array}{l}\text { Library and } \\ \text { Archives Canada }\end{array} & \begin{array}{l}\text { Bibliothèque et } \\ \text { Archives Canada }\end{array} \\ \begin{array}{l}\text { Published Heritage } \\ \text { Branch }\end{array} & \begin{array}{l}\text { Direction du } \\ \text { Patrimoine de l'édition }\end{array} \\ \begin{array}{l}\text { 395 Wellington Street } \\ \text { Ottawa ON K1A ON4 }\end{array} & \begin{array}{l}\text { 395, rue Wellington } \\ \text { Ottawa ON K1A ON4 } \\ \text { Canada }\end{array}\end{array}$

Your file Votre référence

ISBN: 0-494-00801-6

Ourfile Notre référence

ISBN: 0-494-00801-6

NOTICE:

The author has granted a nonexclusive license allowing Library and Archives Canada to reproduce, publish, archive, preserve, conserve, communicate to the public by telecommunication or on the Internet, loan, distribute and sell theses worldwide, for commercial or noncommercial purposes, in microform, paper, electronic and/or any other formats.

The author retains copyright ownership and moral rights in this thesis. Neither the thesis nor substantial extracts from it may be printed or otherwise reproduced without the author's permission.
AVIS:

L'auteur a accordé une licence non exclusive permettant à la Bibliothèque et Archives Canada de reproduire, publier, archiver, sauvegarder, conserver, transmettre au public par télécommunication ou par l'Internet, prêter, distribuer et vendre des thèses partout dans le monde, à des fins commerciales ou autres, sur support microforme, papier, électronique et/ou autres formats.

L'auteur conserve la propriété du droit d'auteur et des droits moraux qui protège cette thèse. $\mathrm{Ni}$ la thèse ni des extraits substantiels de celle-ci ne doivent être imprimés ou autrement reproduits sans son autorisation.
In compliance with the Canadian

Privacy Act some supporting forms may have been removed from this thesis.

While these forms may be included in the document page count, their removal does not represent any loss of content from the thesis.
Conformément à la loi canadienne sur la protection de la vie privée, quelques formulaires secondaires ont été enlevés de cette thèse.

Bien que ces formulaires aient inclus dans la pagination, il n'y aura aucun contenu manquant.

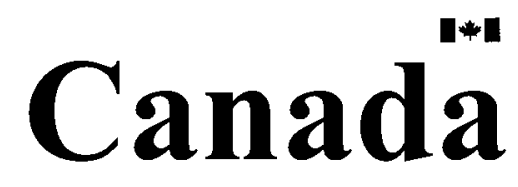




\begin{abstract}
First-generation evaluations of correctional substance abuse programs focus on the ultimate outcomes of recidivism and relapse. Second-generation evaluations have moved to include more aspects of program theory and as a result, assess the attainment of short- and long-term outcomes. The framework for this evaluation integrated the entire program theory of a correctional community-based substance abuse treatment program for male offenders. The main purpose of the evaluation was to expand second-generation work by studying the impact of specific program activities as well as the overall program. Two other objectives of the evaluation were to examine differences between program completers and noncompleters with respect to personal gain and consider program implementation by assessing the program's adherence to the principles of effective correctional treatment.
\end{abstract}

The current evaluation used a quasi-experimental pretest-posttest design. Data were collected from offenders and program staff with several instruments including offender self-reports and staff assessments. The short- and long-term outcomes of interest were knowledge of substance abuse issues, knowledge and ability with prosocial and relapse prevention skills, motivation, and confidence.

Results indicated that the counselling activity produced increases in motivation to participate in treatment and motivation to change substance-abusing behaviour. As hypothesized, the drug and alcohol education activity increased knowledge of substance abuse issues. The cognitive-behavioural program component increased knowledge and ability with prosocial and relapse prevention skills. Findings showed that program completers had a greater increase in knowledge and ability with prosocial and relapse 
prevention skills than noncompleters. Following program completion, offenders also had a greater increase in confidence that they would not use substances. Results demonstrated that the community substance abuse program is an appropriate intervention that adheres to most of the principles of effective correctional treatment. Additional analyses suggested that the best individual predictors of program completion were being low risk, using alcohol rather than drugs, and being 35 years old or older. Recommendations for the program, funding agencies, and program evaluators are derived from these results. 


\section{Acknowledgements}

The successful completion of this dissertation was possible thanks to the contribution of a number of individuals. I am indebted to my supervisor, Dr. Shelley Borys. Shelley, without you, my desire to conduct an evaluation for my dissertation would have been impossible. Your evaluation mentoring both inside and outside academia has been invaluable. I also want to acknowledge my co-supervisor, Dr. Robert Hoge. Thanks for agreeing to take on this position and guiding me through the process.

I want to extend a huge thank you to my defense committee - Dr. Frank Porporino, Dr. Diana Majury, Dr. Adelle Forth, and Dr. Tina Daniels. I couldn't have asked for a better committee! My defense was an amazing experience. Also, I would like to recognize two of my committee members who were major influences in my undergraduate years. Diana and Adelle, the time each of you invested with me to foster my skill and knowledge was exceptional.

I am also grateful to the Executive Director of $\mathrm{MDH}$, Louis Bérubé and his staff. Merci beaucoup, Louis, for your enduring commitment to this project. To the MDH staff and students as well as Lucie Carriere at OCDC, a sincere thank you for your dedication and patience with data collection. Your efforts resulted in data of outstanding quality.

I have been a student at Carleton for a long time, and some wonderful women in the Department of Psychology have continually guided me. I want to thank Etelle Bourassa, Jacquie Capstick, Kim Cook, Lynn Giff, and Natalie Pressburger for always being friendly faces and ready to assist. 
Two other ladies must be mentioned for their constant encouragement, compassion, and camaraderie - my "bestest" girlfriends, Tanya Rugge and Jennifer van de Ven. University wouldn't have been the same without you two! And Jennifer, thank you for your statistical guidance over the years - my ability is truly a reflection of your instruction. I would also like to extend my gratitude to Dr. Jackie Rogers for keeping my body limber enough so I could continue typing and reading.

As for my family, they deserve medals for picking up all my slack! $\mathrm{Da}$, you set a great example of academic achievement and continue to inspire me. Mum, thanks to you, I think I have forgotten how to cook and grocery shop! And Ché, you made me want to be a better writer - although, my writing will never be as articulate as your prose. I also want to thank Barbara and Arnold for always understanding why their son's wife couldn't visit.

And above all, I want to acknowledge my husband. David, you have been relentless with your support. I thank you for making endless trips to the library to photocopy articles for me, always keeping my computer current, and reminding me to make back-ups. I thank you for taking care of me and selflessly putting yourself second. I thank you for always seeing my ability and potential, especially when I didn't. Over the years your mantras have been many, but the one from the Matrix was particularly motivating - "Don't think you will, know you will". You are an incredible human being!

The completion of this dissertation has been quite a journey that has taught me much both academically and personally. And, I think my greatest lesson has been best expressed by Leonard Cohen who wrote, "There is a crack in everything. That's how the light gets in". 


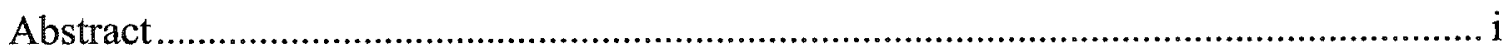

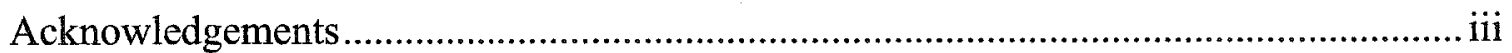

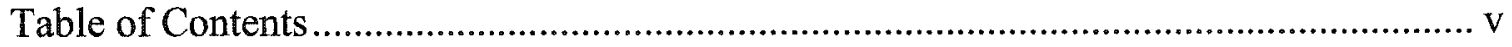

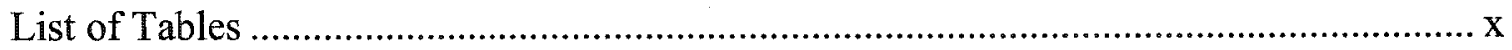

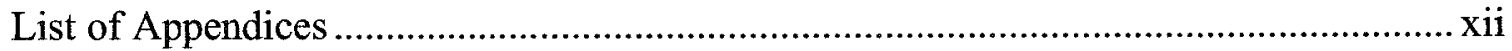

Chapter 1: Establishing the Need for Second-Generation Evaluation of Correctional Community Substance Abuse Programs ................................................................ 1

Prevalence of Substance Abuse ........................................................................... 1

Substance Abuse and Criminal Behaviour ..................................................... 2

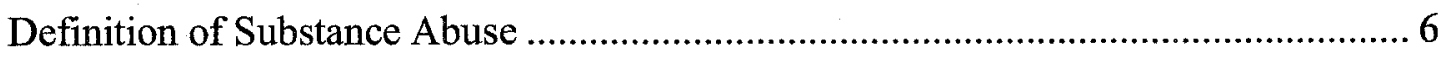

Substance Abuse Treatment within the Criminal Justice System........................... 7

Effectiveness of Correctional Substance Abuse Treatment................................... 9

Effective Correctional Treatment ................................................................. 10

The Generations of Evaluation on Correctional Substance Abuse Programs............ 11

Purpose of the Current Evaluation and Organization of the Literature Review ......... 16

Chapter 2: Multimodal Substance Abuse Treatment ................................................. 18

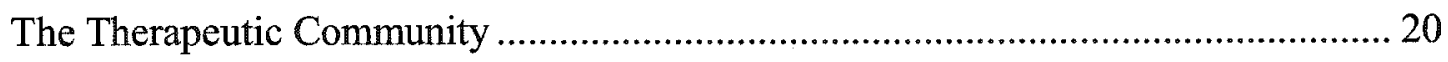

MDH Substance Abuse Treatment Program..................................................... 25

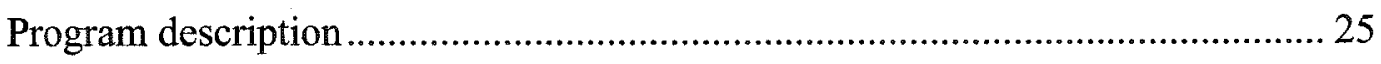

Program design and program theory ........................................................ 27

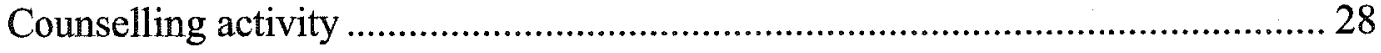

Drug and alcohol education activity .......................................................... 32

Cognitive-behavioural intervention activity ................................................. 36

Differences and similarities between MDH and the TC................................. 43 
Chapter 3: The Principles of Effective Correctional Treatment ................................. 45

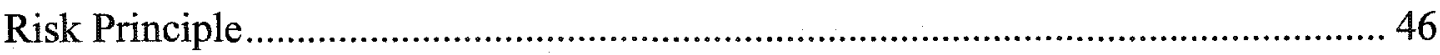

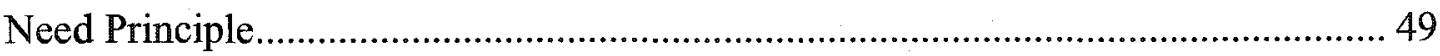

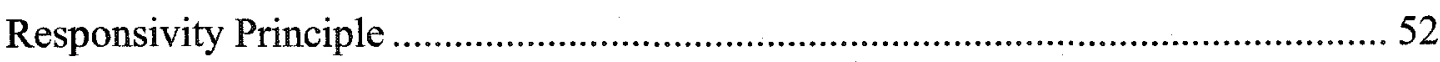

Expansion of the Principles of Effective Correctional Treatment ........................... 53

Multimodal programming ....................................................................... 55

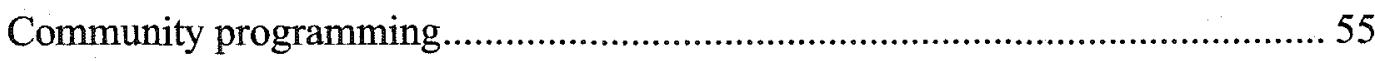

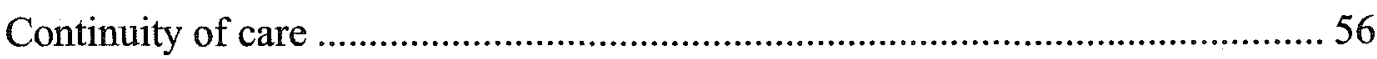

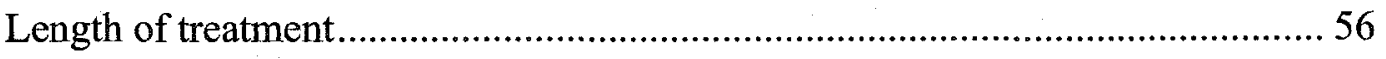

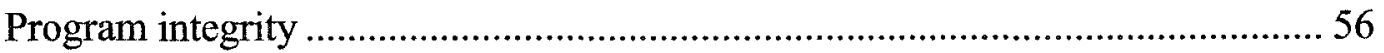

Chapter 4: First- and Second-Generation Substance Abuse Program Evaluations .......... 59

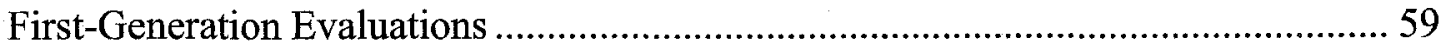

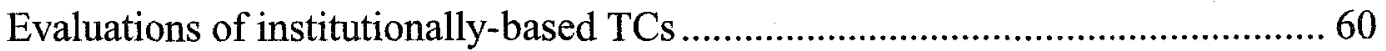

Evaluations of institutionally-based TCs with community aftercare...................6 60

Evaluations of community-based TCs .......................................................6 68

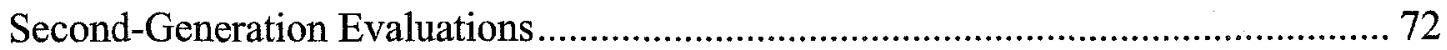

Chapter 5: Summary of Literature Limitations, Hypotheses, and Evaluation Issues ....... 83

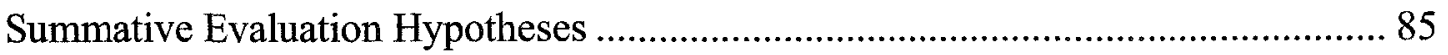

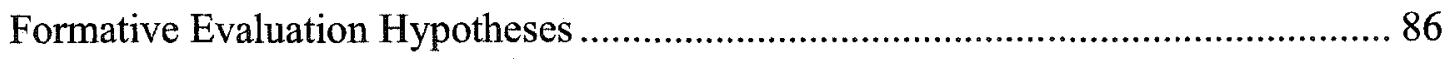

Formative Issues Related to Program Implementation ....................................... 87

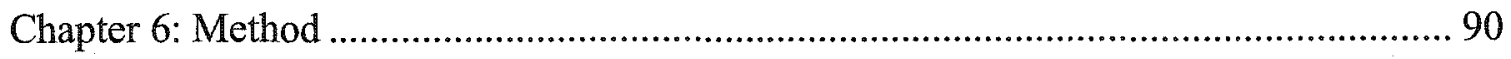

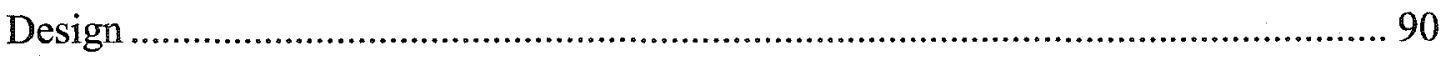

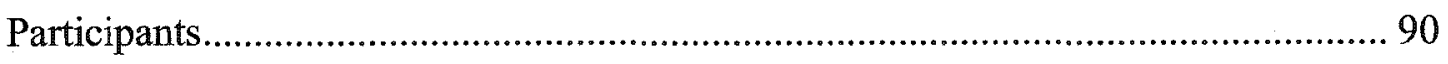

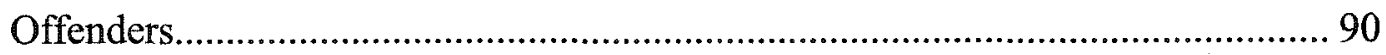

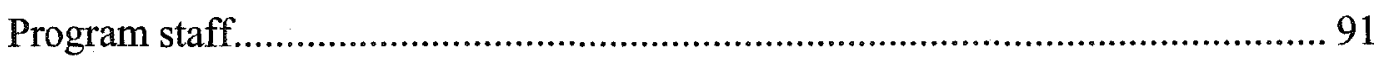

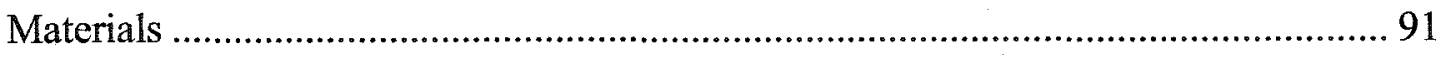

Drug-Taking Confidence Questionnaire .................................................... 91

Readiness to Change Questionnaire - Treatment Version ................................ 93 
Problem Solving Questionnaire ........................................................................ 95

Assertiveness Questionnaire ........................................................................... 96

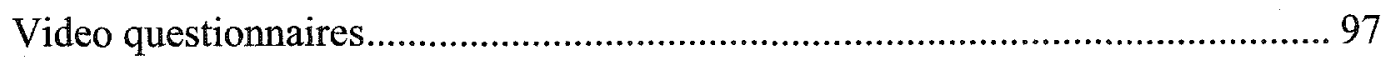

Pre- and postprogram client surveys............................................................... 97

Balanced Inventory of Desirable Responding ................................................. 100

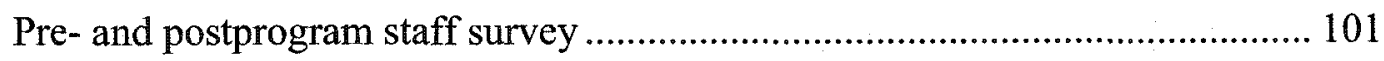

Key informant interviews ........................................................................... 102

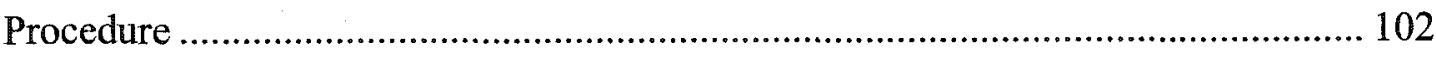

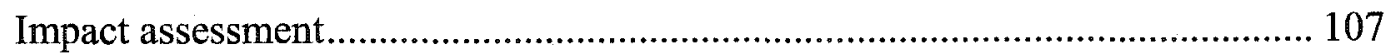

Client and program staff surveys ..................................................................... 110

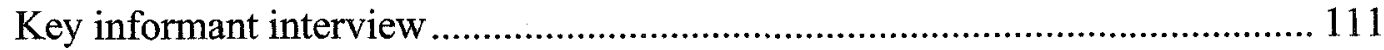

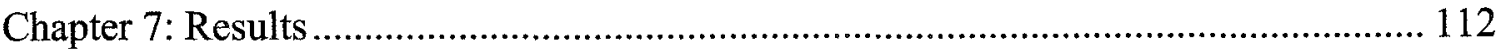

Examination of Results ..................................................................................... 112

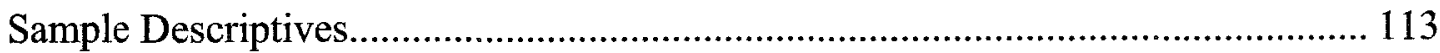

Substance Abuse Descriptives ............................................................................. 118

Changes to the Original Program............................................................................ 121

Missing Data on Noncompleters........................................................................... 123

Internal Consistency and Split-Half Estimates of the Client Survey, Program Staff Survey, and Pre- and Postvideo Questionnaires ...................................................... 126

Hypothesis 1 - Program Completion Will Increase Knowledge of Prosocial Skills 127

Offender self-report .................................................................................. 127

Program staff assessment ............................................................................. 133

Hypothesis 2 - Program Completion Will Increase Knowledge of Relapse Prevention

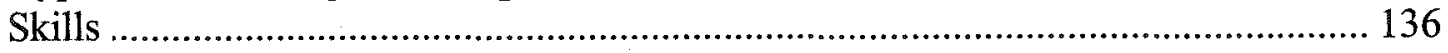

Offender self-report ……………………………………........................ 137

Program staff assessment .............................................................................. 139

Hypothesis 3 - Program Completion Will Increase Ability With Prosocial Skills .. 139

Offender self-report .......................................................................................... 139

Program staff assessment................................................................................ 140 
Hypothesis 4 - Program Completion Will Increase Ability with Relapse Prevention

Skills

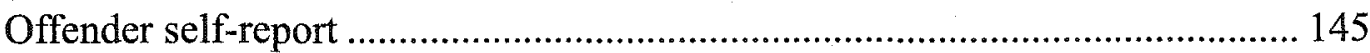

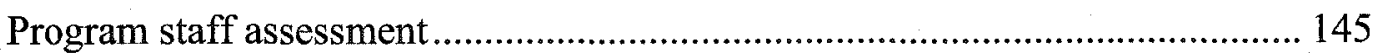

Hypothesis 5 - Program Completion Will Increase Confidence to Not Use Substances

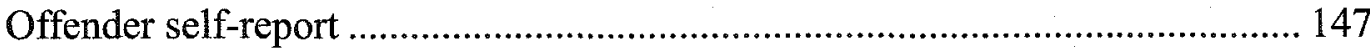

Hypothesis 6 - Knowledge of Substance Abuse Issues Will Increase Following the Drug and Alcohol Education Component of the Program.................................... 153

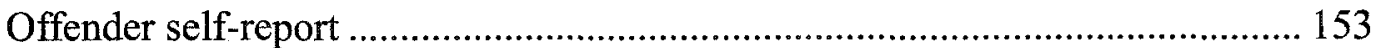

Hypothesis 7 - Motivation to Participate in Treatment Will Increase and Motivation Will Move Across the Stages Following the Initial Counselling Component.......... 154

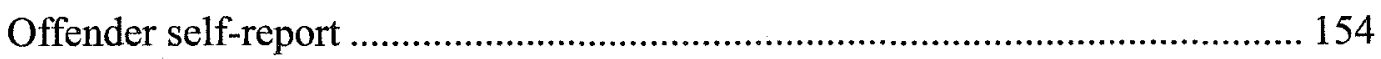

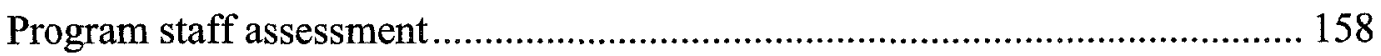

Hypothesis 8 - Knowledge of Prosocial Skills Will Increase Following the Cognitive-

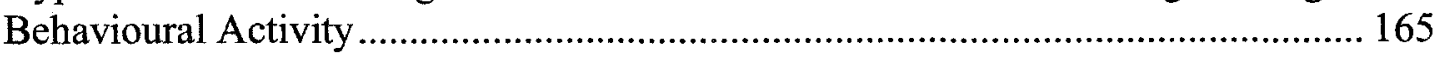

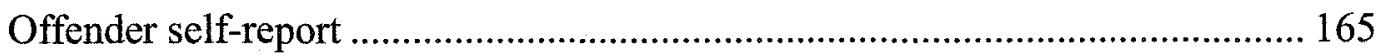

Hypothesis 9 - Knowledge of Relapse Prevention Skills Will Increase Following the

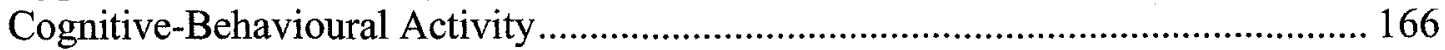

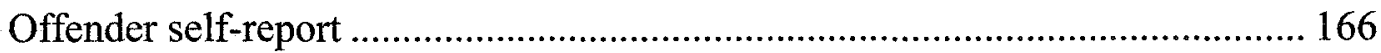

Hypothesis 10 - Ability with Prosocial Skills Will Increase Following the Cognitive-

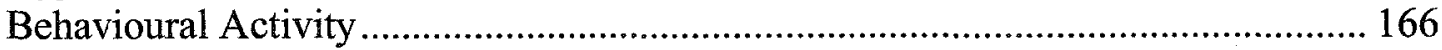

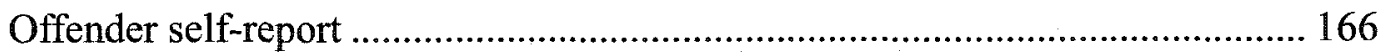

Hypothesis 11 - Ability with Relapse Prevention Skills Will Increase Following the

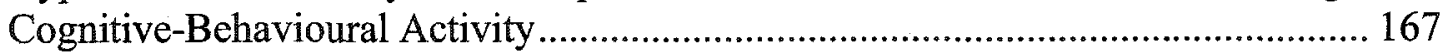

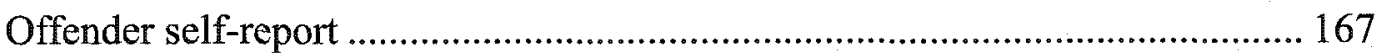

Hypothesis 12 - Self-Improvement Following Cognitive-Behavioural Activity ..... 167

Offender self-report ............................................................................ 167

Hypothesis 13 - Quality of Program Implementation ....................................... 171

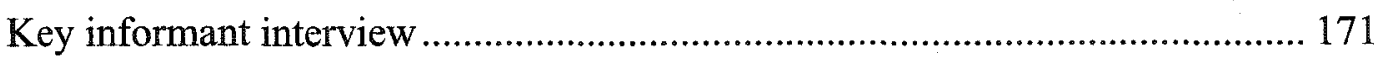

Additional Analyses - Prediction of Program Completion ................................ 172 


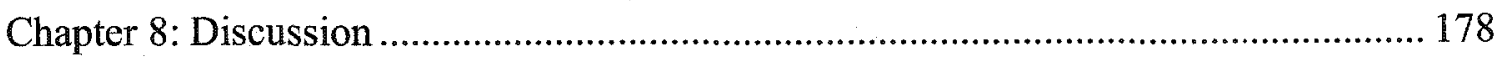

Summary of Findings and Theoretical Linkages ............................................ 178

Knowledge and ability with prosocial and relapse prevention skills................. 178

Confidence to not use substances ............................................................... 180

Influence of the drug and alcohol education activity on knowledge of substance

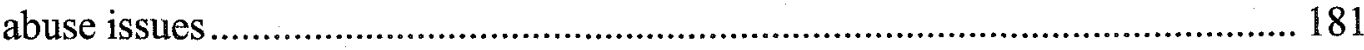

Influence of the counselling activity on motivation to participate in treatment and motivation to change substance abuse behaviour ............................................ 181

Influence of the cognitive-behavioural activity on knowledge and ability of prosocial and relapse prevention skills ........................................................ 184

Self-improvement following the cognitive-behavioural activity ..................... 185

Adherence to the principles of effective correctional treatment....................... 185

Prediction of program completion ............................................................... 186

Implications and Recommendations ......................................................... 187

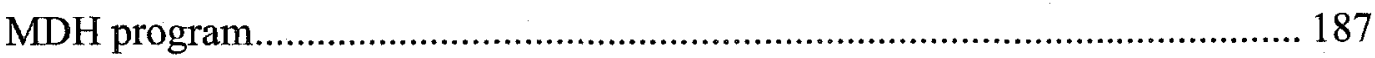

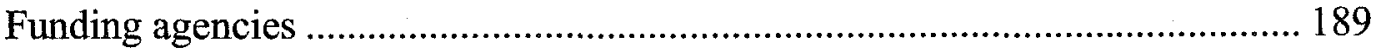

Correctional program evaluators............................................................... 190

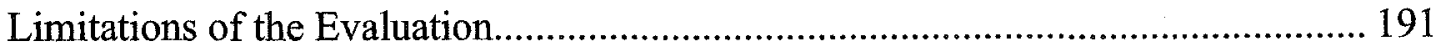

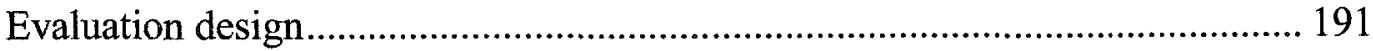

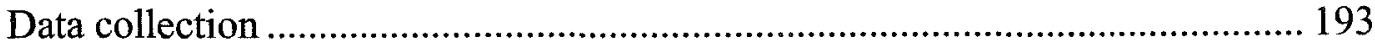

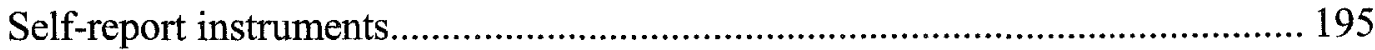

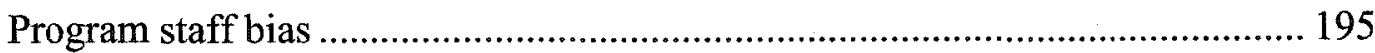

Future Program Evaluation and Research Directions ........................................ 196

Future evaluations of MDH ................................................................ 196

Future directions for correctional substance abuse programming ..................... 198

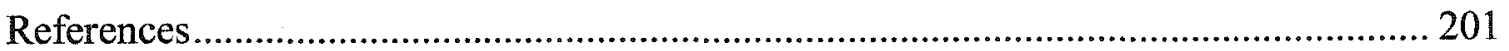




\section{List of Tables}

Table $1 \quad$ Promising and Less Promising Treatment Targets 51

Table 2 Effectiveness of Institutionally-Based TC with Community Aftercare in Reducing Recidivism and Relapse.. 62

Table 3 Effectiveness of Community-Based TC in Reducing Recidivism and Relapse.

Table 4 Summary of Findings from Second-Generation Evaluations................... 75

Table 5 Summary of Evaluation Issues, Questions, Indicators, and Methods ..... 108

Table 6 Sample Descriptives for the Total Sample and for Each Group ............ 115

Table 7 Univariate Results from MANOVA Examining the BIDR SDE and IM Subscales Over Time as a Function of Program Completion.

Table 8 Substance Abuse Descriptives Variables for the Total Sample and for Each Group

Table 9 Percentages of Previous Substance Abuse Treatment Participation and Completion of Substance Abuse Programs for the Total Sample and for Each Group

Table 10 Comparison Between Offenders who Did and Did Not Participate in the Drug and Alcohol Education Activity ....

Table 11 Comparison Between Offenders who Did and Did Not Participate in the Revised Delivery of the Counselling and Cognitive-Behavioural Activities

Table 12 Distribution of Reasons Why Noncompleters Did Not Have Posttesting $(\mathrm{n}=20)$

Table 13 Univariate Results from MANOVA Examining Knowledge of Prosocial Skills as a Function of Program Completion Based on Offender SelfReport

Table 14 Univariate Results for Simple Effects of Test Administration Time at Each Level of Risk

Table 15 Program Staff Assessment on Knowledge of Prosocial Skills by Group Postprogram

Table 16 Offender Self-Report on Knowledge of Relapse Prevention Skills by Group 
Table 17 Univariate Results from MANCOVA Examining Ability with Prosocial Skills as a Function of Program Completion Based on Offender SelfReport.

Table 18 Univariate Results from MANCOVA Examining Ability with Prosocial Skills as a Function of Program Completion Based on Program Staff Assessment.

Table 19 Univariate Results from ANCOVA Examining Ability with Relapse Prevention Skills as a Function of Program Completion Based on Offender Self-Report. 146

Table 20 Correlation Matrix for the Pretest Measures of the DTCQ $(n=81)$ 148

Table 21 Univariate Results from MANCOVA Examining Increases in Confidence as a Function of Program Completion Based on Offender Self-Report . 151

Table 22 Univariate Results from ANCOVA Examining Increased Motivation to Participate in Treatment Following the Initial Counselling Activity Based on the Client Survey.....

Table 23 Univariate Results from MANCOVA Examining Motivation to Change Based on the RCQ-TV.

Table $24 \quad$ Univariate Results from MANCOVA Examining Motivation to Participation in Treatment and Change Substance Abuse Behaviour Based on Program Staff Assessment

Table 25 Results from Repeated Measures Analysis Examining Self-Improvement Following the Three Program Activities Measured Based on Offender Self-Report.

Table 26 Summary of Direct Logistic Regression Analysis for Variables Predicting Program Completion $(\mathrm{n}=58)$ 174

Table 27 Classification Results Using Discriminant Function Analysis $(n=58) . .176$ 
Appendix A Logic Model: MDH Substance Abuse Treatment Program ...................... 229

Appendix B Original Logic Model: MDH Substance Abuse Treatment Program....... 231

Appendix C Drug-Taking Confidence Questionnaire .............................................. 233

Appendix D Readiness to Change Questionnaire - Treatment Version (Alcohol Use) 238

Appendix E Readiness to Change Questionnaire - Treatment Version (Drug Use) .... 241

Appendix F Problem-Solving Questionnaire …………………………………......... 244

Appendix G Assertiveness Questionnaire.................................................................... 247

Appendix H Shame Video Questionnaire ………………………………………..... 250

Appendix I Anger Video Questionnaire................................................................... 252

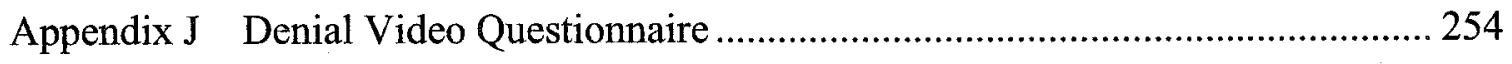

Appendix K My Father's Son Video Questionnaire ................................................... 256

Appendix L Preprogram Client Survey ……………………….............................. 258

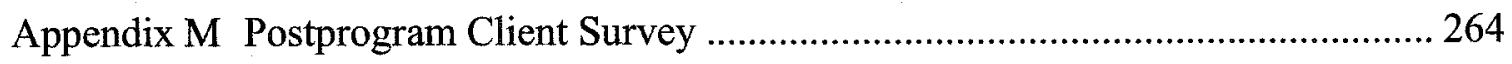

Appendix N Balanced Inventory of Desirable Responding ......................................... 273

Appendix O Pre- and Postprogram Staff Survey ...................................................... 276

Appendix P Key Informant Interview for Executive Director ..................................... 280

Appendix Q Key Informant Interview for Counsellors ................................................ 283

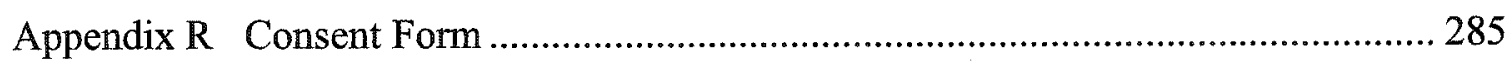

Appendix S Debriefing Form ................................................................................... 287

Appendix T Data to Collect for the Methodologies .................................................... 289

Appendix U Additional Tables for Results Section...................................................... 291

Appendix V Profile Plots for Results Section............................................................. 298 
Chapter 1: Establishing the Need for Second-Generation Evaluation of Correctional

\section{Community Substance Abuse Programs}

Correctional populations have been under scrutiny for over a century. This continual investigation has uncovered a multitude of deficits among offenders. A problem plaguing many male offenders is substance abuse. Research studies have found that approximately $70 \%$ of Canadian male offenders have substance abuse problems (Boland, Henderson, \& Baker, 1998; Grant, 2001; Langevin, Blanchet, \& Long, 1999; Lightfoot, 2001; Robinson, Porporino, \& Millson, 1991; Roy, 2001; Weekes, Moser, \& Langevin, 1997). This epidemic within the male offender population is also manifest in other countries, including the United States (Centre on Addiction and Substance Abuse, 1998; US Department of Justice, 1997; US Department of Justice, 1998a), New Zealand (Morris, 2001), and Australia (d'Orban, 1991).

\section{Prevalence of Substance Abuse}

A recent profile of the Canadian federal inmate population revealed that $59 \%$ of male offenders abuse alcohol and 67\% abuse drugs (Boe, Nafekh, Vuong, Sinclair, \& Cousineau, 2003). Similar statistics were reported in an earlier Canadian study, when severity of abuse was examined. ${ }^{1}$ Severity of abuse ranged from low to severe. In this report, it was estimated that $48 \%$ of male offenders had a drug problem of some severity, and approximately $52 \%$ had some severity of alcohol dependence (Robinson, Porporino,

\footnotetext{
1 In this study 503 male offenders, who went through the intake process were examined. The intake process, which takes place in all federal institutions in Canada, is known as the Computerized Lifestyle Assessment Inventory (CLAI; Robinson, Fabiano, Porporino, Millson, \& Graves, 1993). Within the CLAI are several substance abuse assessment tools, including the Alcohol Dependence Scale (ADS; Skinner \& Horner, 1984), the Problems Related to Drinking Questionnaire (PRD; Robinson et al., 1993), and the Drug Abuse Screening Test (DAST; Skinner, 1982).
} 
\& Millson, 1991). Identical patterns of drug abuse and alcohol dependence were recorded in a sample of 4,941 federal male offenders, who went through the same intake process (Weekes, Fabiano, Porporino, Robinson, \& Millson, 1993).

In addition to the identification of substance abuse problems, substance consumption rates among offender populations have been investigated. In a profile study of 8,598 Canadian male offenders admitted to federal institutions over a two-year period, it was found that six months prior to arrest $19 \%$ of offenders used drugs everyday or almost everyday, $13 \%$ used alcohol everyday or almost everyday, and $6 \%$ used both drugs and alcohol everyday or almost everyday (Brochu et al., 2001). This same study found that six months prior to arrest, $34 \%$ of offenders reported using drugs at least once a week, 44\% disclosed weekly consumption of alcohol, and 18\% said they used both drugs and alcohol at least once a week. Another Canadian study examining patterns of alcohol and drug use among federal male offenders found that $37 \%$ used drugs regularly six months prior to arrest, ${ }^{2}$ and 50\% consumed alcohol regularly (Robinson et al., 1991). Similarly, $57 \%$ of federal inmates in the United States reported regular drug use (Mumola, 1999), and 62\% of convicted jail inmates disclosed regular alcohol consumption (US Department of Justice, 1998b).

\section{Substance Abuse and Criminal Behaviour}

Given the prevalence of substance abuse problems among offenders, it is not surprising, then, that numerous empirical studies illuminate the association between substance use and criminal activity (Andrews \& Bonta, 1998, 2003; Boland et al., 1998;

2 Regularly is defined as once a week or more (Robinson et al., 1991; US Department of Justice, 1998a). 
Brochu et al., 2001; d'Orban, 1991; Glider, Mullen, Herbst, Davis, \& Fleishman, 1997; Robinson et al., 1991; US Department of Justice, 1998b). A variety of investigations have demonstrated this association.

Research on substance use prior to committing offences has revealed that more than half of all offenders are under the influence of either drugs and/or alcohol at the time of their offence (Brochu et al., 2001; Grant, 2001; Lightfoot \& Hodgins, 1988; Lipton, 1995; Robinson et al., 1991; Roy, 2001; Siegal et al., 1999; Wexler, Falkin, Lipton, \& Rosenblum, 1992; US Department of Justice, 1998a, 1998b). For example, Brochu et al. found that $16 \%$ of Canadian federal inmates had been under the influence of drugs at the time they committed their offence on their current sentence, $21 \%$ reported using alcohol on the day of their offence, and $13 \%$ disclosed that they had been under the influence of both alcohol and drugs while committing their index offence. Similarly, in a survey of 275 Canadian federal offenders, Lightfoot and Hodgins reported that $79 \%$ of the offenders reported having used alcohol and/or drugs on the day of their offence. Specifically, $13 \%$ of the sample used drugs, $26 \%$ consumed alcohol, and $40 \%$ used both drugs and alcohol.

Further analyses with Canadian federal offenders have indicated that the relationship between substance use and crime increases with severity of the substance abuse problem (Weekes et al., 1993). Specifically, half of offenders with low severity substance abuse problems (51\%) reported using substances on the day they committed their index offence, $80 \%$ of offenders with moderate problems reported substance use, and $89 \%$ with a substantial problem disclosed use. Almost all offenders with severe 
substance abuse problems (97\%) admitted to using substances on the day they committed their offence.

Research on substance use and its relationship to different types of crime has demonstrated that use is tied to a broad range of criminal behaviour, including violent offences, property offences, drug offences, and sex offences (Brochu et al., 2001; Lightfoot \& Hodgins, 1988; Millson et al., 1993; Robinson et al., 1991; US Department of Justice, 1998b; Weekes et al., 1993). Although there are instances where drug users engage in violent crime, such as robbery, drug use is mostly linked to property crimes, such as break and enters, theft, and fraud (Brochu et al., 2001; Millson et al., 1993; US Department of Justice, 1998b; Weekes et al., 1993). In contrast, violent offences are more often committed while under the influence of alcohol (Brochu et al., 2001; Robinson et al., 1991; US Department of Justice, 1998b).

A line of evidence illustrating the relationship between substance abuse and criminal behaviour is the risks/needs literature (Andrews, 1995; Andrews \& Bonta, 1998, 2003). Through this research, substance abuse has been identified as a risk factor, or criminogenic need factor, along with other variables. Meta-analytic studies have found that the most validated risk factors in the research literature, referred to as the "Big Eight", are: antisocial attitudes, antisocial associates, a history of antisocial behaviour, antisocial personality pattern, problematic circumstances at home (family/marital), problematic circumstances at school or work, problematic leisure circumstances, and substance abuse (Andrews \& Bonta, 2003). Thus, risk factors, including substance abuse, can be used to predict the likelihood of an offender committing another offense. Over the last decade, meta-analyses have been conducted examining predictors of general and 
violent reoffending among various male offender populations. These meta-analyses identified substance abuse as a significant predictor of both general and violent recidivism (e.g., Bonta, Pang, \& Wallace-Capretta, 1995; Dowden \& Brown, 2002; Gendreau, Goggin, \& Little, 1996).

The presentation of research on substance use/abuse and criminal behaviour is neither intended to suggest that substance abuse is the only correlate to criminal behaviour nor that there is a direct link between substance use and crime. As mentioned previously, substance abuse is one of many factors found to be associated with criminal behaviour and recidivism. Furthermore, the relationship between substance use and crime is quite complex, and seems to involve interrelationships between biological, psychological, and social variables (d'Orban, 1991; Hollin, 1994; Lightfoot, 1997, 2001).

Attempts have been made to explain the substance abuse/crime relationship, and to determine whether substance abuse leads to criminal behaviour or whether substance abuse is part of the lifestyle of individuals who commit crime (Lightfoot, 2001). It has been suggested that both scenarios occur (Lightfoot, 2001; Tonry, 1990). Lightfoot believes there are two types of individuals with substance abuse problems involved in the criminal justice system. The first proposed type of individual is described as a "primary criminal", defined as an individual with Conduct Disorder who is predisposed to abuse substances as well as engage in criminal behaviour. The second type of individual is referred to as a "primary substance abuser". A primary substance abuser is depicted as an individual who develops a substance abuse disorder, and then begins to engage in criminal behaviour to support the dependency. 


\section{Definition of Substance Abuse}

Two terms related to substance use (i.e., abuse and dependence) were presented in this review without any attempt to articulate the distinction between the two.

Unfortunately, within the correctional substance abuse literature, these terms are often used interchangeably, and not clearly operationalized. One of the most widely used methods for identifying substance abuse disorders is provided in the Diagnostic and Statistical Manual of Mental Disorders (DSM-IV; American Psychiatric Association, 1994). Within the DSM-IV, substance abuse disorders subsume two diagnoses, an abuse diagnosis, and a dependence diagnosis.

Substance abuse is considered "a maladaptive pattern of substance use manifested by recurrent and significant adverse consequences related to the use of substances" (American Psychiatric Association, 1994, p. 182). The consequences can be physically hazardous (e.g., driving a car while under the influence of a drug), social or interpersonal (e.g., arguments with a spouse about using substances), legal (e.g., committing assault and battery), and/or occupational (e.g., repeated absences from work). To be diagnosed with substance abuse these problems must occur regularly over a continuous 12 -month period (American Psychiatric Association, 1994).

Generally, substance dependence is a more severe form of substance abuse disorder, and is described in the DSM-IV as "a cluster of cognitive, behavioural, and physiological symptoms indicating that the individual continues use of the substance despite significant substance-related problems" (p. 176). In general, substance dependence is associated with more physiological symptoms or consequences of use than 
substance abuse. Some of the substance-related problems include: tolerance, ${ }^{3}$

withdrawal, ${ }^{4}$ substantial amounts of personal time spent getting and using substances as well as recovering from use of substances, continued use despite psychological or physical problems, regular expression of wanting to better control use, many failed attempts to reduce or stop use, and important social, occupational, or recreational activities are stopped or decreased because of use.

\section{Substance Abuse Treatment within the Criminal Justice System}

The criminal justice system recognizes that substance abuse is associated with criminal activity. Consequently, while under the supervision of the correctional system, it is recognized that offenders whose substance abuse is related to their criminal behaviour should participate in substance abuse treatment. This participation in substance abuse programming is seen as a potential tool to reduce recidivism (Broome, Knight, Hiller, \& Simpson, 1996; Murray, 1996; Lightfoot, 2001; Swartz, Lurigio, \& Slomka, 1996; Wexler, 1995; Wexler, Williams, Early, \& Trotman, 1996). As a result, programming strategies have been integrated into correctional mandates. Across North America, various substance abuse treatment approaches have been developed and implemented in both federal institutions and community correctional facilities. To illustrate the variety of interventions in use, five treatments are briefly described:

(1) Individual counselling is one-on-one counselling between an offender and counsellor. Individual counselling deals with issues affecting an offender, and

\footnotetext{
3 Tolerance refers to the increased amounts of substance required to achieve the desired effect or the diminished effect with regular use of the same dose (American Psychiatric Association, 1994).

4 Withdrawal is a substance-specific syndrome following cessation of or reduction in intake of a substance (American Psychiatric Association, 1994).
} 
tends to be supportive as well as confrontational (Lipton, Falkin, \& Wexler, 1992; Ross \& Lightfoot, 1985).

(2) Cognitive training strives to enhance an offender's cognitive development, improve problem-solving skills, and teach more effective interpersonal reasoning strategies. Cognitive-behavioural and/or social learning techniques are often used to facilitate cognitive training (Correctional Service of Canada, 1996a; Ross \& Lightfoot, 1985).

(3) Self-help programs, such as Alcoholics Anonymous (AA) and Narcotics Anonymous (NA) insist on a substance-free lifestyle. To assist offenders with achieving life-long abstinence, self-help programs incorporate an extensive support system (Wexler, 1995). Paramount to this type of treatment is the philosophy of "twelve steps", which suggests that the "disease" of substance abuse can only be overcome with personal recognition of an inherent intolerance to alcohol and/or drugs (Lipton et al., 1992; Weekes et al., 1997; Wexler, 1994).

(4) Drug and alcohol education programs attempt to increase an offender's knowledge of problems associated with drug and alcohol use to change attitudes towards the use of these substances. Generally, educational programs are integrated into multimodal substance abuse interventions, such as therapeutic communities (Correctional Service of Canada, 1996a; Lipton et al., 1992; Murray, 1996).

(5) A therapeutic community (TC) is a treatment program that creates a learning environment using peers and staff as role models. This type of treatment 
program incorporates several treatment modalities with an emphasis on group therapy (Murray, 1996). It should be noted that a therapeutic community could incorporate all of the interventions listed above.

\section{Effectiveness of Correctional Substance Abuse Treatment}

To further support the correctional system's decision to adopt substance abuse programming for offenders, an abundance of outcome research has been completed that measures the impact of this type of rehabilitation on recidivism. Recidivism is the most widely used outcome measure in evaluations of general correctional programs, including substance abuse programs. This focus on recidivism stems from both the correctional strategy to reduce reoffending through the implementation of rehabilitative programming (Correctional Service of Canada, 1998a; Lipton, 1995; US Department of Justice, 1995) and the empirical literature suggesting treatment is an essential element for reductions in recidivism (Andrews \& Bonta, 1998; Andrews et al., 1990; Lipton, 1995; Lösel, 1995; McGuire \& Priestly, 1995). Thus, for policy makers in the criminal justice forum, the most critical and most often the only criterion for evaluating substance abuse programs is recidivism (Swartz et al., 1996).

Outcome evaluations have repeatedly demonstrated that participation in certain substance abuse treatment programs can significantly reduce future criminal activity among offender populations ( $\mathrm{T}^{3}$ Associates Inc., 1999a, 1999b, 1999c; Millson, Weekes, \& Lightfoot, 1995; Lipton, 1998; Pearson \& Lipton, 1999). Indeed, a meta-analysis by Andrews, Dowden, and Gendreau (as cited in Andrews and Bonta, 2003) on the literature of the effects of human service in a justice context found a mean effect size of $r=.11$ for substance abuse that was targeted as a criminogenic need in treatment. Using the 
Binomial Effect Size Display (BED, Andrews \& Bonta, 2003), the mean effect size translated into a recidivism rate of $55.5 \%$ in the comparison group compared to $44.5 \%$ in the treatment group.

It has been recognized that reduced recidivism is not the only outcome of successful substance abuse treatment $\left(T^{3}\right.$ Associates Inc., 1999a). Thus, substance abuse program evaluations have also measured reductions in substance use and/or relapse among offenders. Even though fewer studies have examined this treatment outcome, results have demonstrated that participation in substance abuse treatment significantly reduces drug and alcohol relapse rates among offenders (Glider et al., 1997; Inciardi, Martin, Butzin, Hopper, \& Harrison, 1997; Pelissier et al., 1998, 2000; Wexler et al., 1996). In fact, a meta-analysis of the literature on the efficacy of relapse prevention with nonoffender samples found reductions in substance use, with a mean effect size of $r=$ .14 (Irvin, Bowers, Dunn, \& Wang, 1999). Using the BED, the mean effect size translates into a relapse rate of $57 \%$ in the comparison group compared to $43 \%$ in the treatment group.

\section{Effective Correctional Treatment}

Empirical evidence demonstrates not only that substance abuse programming can reduce recidivism and relapse rates, but it also shows that specific elements of correctional programming can influence the likelihood of reoffending. Research has identified several principles of effective correctional treatment, including the principles of risk, need, and responsivity (Andrews, 1995; Andrews \& Bonta, 1998, 2003; Andrews, Bonta et al., 1990; Andrews et al., 1990). In general, treatment programs that incorporate these principles have been shown to be more successful in reducing recidivism than those 
programs that do not (Andrews et al., 1990; Dowden, 1998; Dowden \& Andrews, 1999; Lipsey, 1995; Lösel, 1995). More detail on the principles of risk, need, and responsivity as well as the additional principles of effective correctional treatment is presented in chapter 3.

The Generations of Evaluation on Correctional Substance Abuse Programs

The previously discussed research on the effectiveness of correctional substance abuse treatment examines the impact of substance abuse treatment on recidivism and relapse rates. Within the program evaluation literature, these evaluations would be categorized as summative. Summative evaluations have been defined as investigations of program performance through the achievement of program goals (Rossi, Freeman, \& Lipsey, 1999). It is recognized that the achieved goals examined in a summative evaluation are the result of the entire program. For the purpose of the current evaluation, correctional evaluations examining recidivism and relapse as outcome measures are categorized as "first-generation" evaluations. These evaluations are deemed to be firstgeneration because of their summative perspective and exclusive focus on examining the ultimate program goal (i.e., reduced recidivism and/or relapse), a goal that results from the overall program..$^{5}$

To date, evaluations have focused on recidivism and relapse as outcome measures and yet, program effectiveness can be assessed by other variables. Restricting definitions of effectiveness to recidivism and relapse is considered limiting (Serin, 2001). Indeed,

5 The ultimate outcome of a program can be defined as the desired changes in a participant's (offender's) behaviour(s) resulting from the overall program. This final program outcome can take an extended period of time to be realized (Wong-Reiger \& David, 1995). In addition, it is subject to influences external to the program, and can be reflective of more strategic issues (Treasury Board Secretariat, 2001). 
summative evaluation precludes examination of the entire chain of events based on a program's logic or rationale, ${ }^{6}$ and omits the incorporation of long-term goals, ${ }^{7}$ such as achievement of skill.

The measurement of short-term goals, ${ }^{8}$ such as knowledge gain occurs in a formative evaluation, ${ }^{9}$ rather than a summative evaluation. Very few formative evaluations have been undertaken with correctional substance abuse programs (Lösel, 1995; Serin, 2001). Nonetheless, formative evaluation serves several beneficial functions.

First, formative evaluation has been referred to as "the measurement of treatment components" (Etheridge \& Hubbard, 2000, p. 1766). Given that many first-generation evaluations were completed on therapeutic communities, a multimodal treatment, the summative nature of the evaluations did not permit assessments of the various treatment components incorporated into this type of program. With multimodal substance abuse treatment programs, an examination of the overall program effect leaves unanswered questions about the effect and value of the individual program components (Ethridge \& Hubbard, 2000; Palmer, 1995). The short-term goals examined in a formative evaluation are the outcomes most closely linked to a specific treatment component of a program. Thus, evaluation of the short-term goals distinguishes the effects of individual treatment

\footnotetext{
6 Program logic or rationale is also known as program theory. Program theory is "the set of assumptions about the manner in which the program relates to the social benefits it is expected to produce and the strategy and tactics the program has adopted to achieve its goals and objectives" (Rossi et al., 1999, p. 78). 7 Long-term goals are changes in a program participant that occur once short-term goals have been met. Long-term goals tend to be influenced by more than one program activity, and unlike the short-term outcomes, long-term outcomes are met over a longer duration of time (Rossi et al., 1999; Treasury Board Secretariat, 2001).

8 Short-term goals are changes in a program participant as a result of participation in an activity with the change occurring soon after the activity is performed. Short-term goals tend to be the result of a specific program activity (Rossi et al., 1999; Treasury Board Secretariat, 2001).

Formative evaluation is quite similar to process evaluation. Formative evaluation incorporates process theory into its design. Process theory is a set of assumptions about how a program is supposed to be organized and delivered (Rossi et al., 1999).
} 
components from the overall program effect, which can provide additional formative information. For example, examination of individual treatment components may reveal some program components that are more helpful than others.

Second, formative evaluation allows for the examination of the entire chain of events based on the program's theory. The information generated from this type of evaluation provides insight into which variables contribute to program failures and successes (Etheridge \& Hubbard, 2000; Judd \& Kenny, 1981; Staton, Leukefeld, Logan \& Purvis, 2000; Scarpitti, Inciardi, \& Pottieger, 1993). This kind of information is beneficial to programs because it can complement summative results by providing possible explanations for unanticipated findings, and it can be used for program improvement. For instance, if an evaluation finds that offenders did not increase their ability with relapse prevention skills, knowing that they did not increase their knowledge in this area may help to explain the finding with respect to their ability. Furthermore, if a substance abuse program does not achieve reduced recidivism, knowing the program design did not adhere to the need principle may provide an explanation of the program's inability to reduce reoffending, and highlight a component of the program that requires amendment.

Finally, formative evaluation provides a mechanism for examining client perceptions (Broome et al., 1996). Program participants offer a unique perspective, since they are the only individuals who actually participate in the program. The examination of client perception allows for judging the acceptability of the program in the eyes of clients as well as the perceived effectiveness of the program (Chan, Sorensen, Guydish, Tajima, 
\& Acampora, 1997; Conners \& Franklin, 2000; Field, 1989; Glider et al., 1997).

Furthermore, this client information can be used to inform program modifications.

Given the narrow focus of first-generation evaluation, researchers have suggested a need for a next generation of evaluation (Etheridge \& Hubbard, 2000; Serin, 2001). Second-generation evaluation research does exist, in which additional summative issues as well as formative issues have been incorporated into the evaluation design. Through the addition of summative questions addressing issues, such as knowledge acquired to deal with substance abuse problems, second-generation evaluation has broadened the definition of program effectiveness from the one traditionally used (i.e., recidivism and/or relapse). However, this generation of evaluation is in its infancy.

Of the second-generation evaluations that have been completed, none have been designed with the entire program theory as a reference point. By not addressing the entire program theory, summative issues permitting the assessment of more than just the ultimate program goal are not integrated into the methodology. Consequently, knowledge has not been generated on the utility of individual program activities as well as the attainment of overall program outcomes. The inclusion of formative issues, such as program design, delivery, and client perspectives into second-generation evaluation has also been extremely limited and in some cases nonexistent. The measurement of formative issues is needed in evaluation to determine how correctional substance abuse treatment programs work or do not work. Without this information on the functioning of a program, not only is it challenging to generate possible explanations for program outcomes, but also it is difficult to make decisions regarding program improvement. 
The expansion of second-generation program evaluation is critical for the evolution of correctional substance abuse programming and program evaluation. As well, it will benefit correctional decision-makers who have begun to invest substantial resources in community programming within the Canadian federal correctional system. It is recognized that correctional community programming suffers from a lack of federal guidance and investment. Lockwood, Inciardi, Butzin, and Hooper (1997) return attention to this point in their review of the correctional therapeutic community continuum by quoting Wexler and Williams who stated, "The connection between rehabilitation efforts in prison and the process of integration into society after release is probably one of the most feeble links in the criminal justice system" (p. 87).

Over the last few years, the Correctional Service of Canada (CSC) has started to revitalize community programming by investing in the development of a correctional community-programming infrastructure (Correctional Service of Canada, 1999, 2000; Motiuk, Boe, \& Nafekh, 2002, 2003). Although community programming, such as substance abuse treatment currently exists in communities across the country, program development and implementation have been the responsibility of independent community organizations. As a result, community programming is often not entirely reflective of the treatment structure utilized within the federal correctional system. In addition, little evaluation of these programs has been completed, which community program directors believe is due to the limited financial and human resources found within most community spheres (L. Bérubé, personal communication, December 16, 2002; D. Wadel, personal communication, September 18,2001 ). Evaluation of existing community programs would be valuable for the establishment of an effective and efficient community substance abuse 
programming infrastructure as well as for the determination of the integrity of these community programs.

\section{Purpose of the Current Evaluation and Organization of the Literature Review}

With the marked need for evolution of correctional substance abuse treatment evaluation as well as the need for assessment of correctional community substance abuse treatment programs, the purpose of the current evaluation was to advance the secondgeneration by addressing the limitations of past second-generation evaluations. This second-generation program evaluation was conducted on the Maison Decision House $(\mathrm{MDH})$ substance abuse treatment program. As a relatively new correctional community substance abuse program, MDH was particularly interested in the evaluation of its effectiveness and efficiency in order to improve and advance its treatment service. MDH is a multimodal program, which is a treatment approach common to other correctional community substance abuse programs. Given MDH's representativeness, the evaluation completed on this program could serve as a template for other correctional community substance abuse programs.

The next chapter of the literature review begins with an overview of the multimodal TC treatment approach, followed by a more specific description of the MDH substance abuse treatment program. Synopsis of the TC model is beneficial, because it details a predominant substance abuse treatment approach, which is the focus of the majority of program evaluations completed to date. The TC approach is also reviewed since the MDH program most closely resembles a therapeutic community. Provision of the MDH program description not only adheres to the suggested structure of a formative evaluation (Etheridge \& Hubbard, 2000; Staton, Leukefeld, Logan \& Purvis, 2000; 
Scarpitti, Inciardi, \& Pottieger, 1993), but it also addresses the need for substance abuse program evaluations to provide sufficient program detail. Most substance abuse program evaluations consist of vague descriptions that hinder the production of literature reviews, experimental replication (Gaes, 1998), and applications to the field. ${ }^{10}$

Following the program summaries, chapter 3 presents the original principles of effective correctional treatment. In addition to the three initial principles, other more recent principles are outlined. The presentation of this literature is not only intended to demonstrate current understanding of "what works" in correctional settings, but also to illustrate how these principles can serve as formative issues for assessing the appropriateness of the MDH program design and delivery.

The subsequent chapter of the literature review outlines the first-generation substance abuse program evaluations to illustrate the evaluation approach of this generation. Next, the small number of second-generation evaluations is outlined. Review of the evaluation literature functions to refine the specific questions addressed in the current evaluation, and to identify gaps in the literature that are addressed. Chapter 5 concludes the literature review with a summary of specific hypotheses and issues.

\footnotetext{
10 It should be noted that in this literature review several meta-analyses are reviewed and relevant correlation coefficients presented. According to Cohen's (1992) conventions, an $r=.10$ is a small effect, an $r=.30$ is a medium effect, and an $r=.50$ is a large effect.
} 


\section{Chapter 2: Multimodal Substance Abuse Treatment}

Many theories have been advanced to account for substance abuse (Miller \& Kurtz, 1994; Ogborne, 1997; Rankin, 1978). A number of theories have focused on genetic and other biological factors as the primary catalyst of abuse, while some emphasize psychological factors and others focus on social and environmental variables (Ogborne, 1997; Rankin, 1978). Assessment of this broad range of theories exemplifies the multifaceted nature of substance abuse and consequently, many experts accept that substance abuse is a complex interaction of biological, psychological, and social structures and processes (Arif \& Westermeyer, 1988; Soden \& Murray, 1997).

The cause of substance abuse is not only a complex phenomenon, but so too is the treatment of substance abuse. Within the literature, ample evidence exists to suggest substance abusers suffer from a variety of life problems in addition to their abuse of alcohol and/or drugs (Cahalan, 1978; Cahalan \& Cisin, 1976; Lightfoot \& Hodgins, 1988; Miller, 1989; World Health Organization, 1980). Furthermore, it is recognized that these life problems may contribute to problematic substance use (Ogborne, 1997). Several factors have been identified in the literature as problem areas for substance abusers, such as education, employment, marital and family relations, social skills, and coping skills (Correctional Service of Canada, 1996a; Hodgins \& Lightfoot, 1989; Langevin, 1999; Lockwood et al., 1997; Miller \& Hester, 1986; Monti, Abrams, Kadden, \& Cooney, 1989; Sobell \& Sobell, 1993). In accordance with the literature outlining the range of problem areas substance abusers can have, as well as the acceptance that substance abuse is a multifaceted interaction of biological, psychological and socialenvironmental structures and processes, a multitude of substance abuse treatment 
programs have been developed to address problematic substance use as well as the various deficits substance abusers can experience (Correctional Service of Canada, 1996a; Ross \& Lightfoot, 1985).

Often treatment is delivered to program participants as a stand-alone program. However, with the known complexity of substance abuse, treatment is often designed as a multimodal program (Ross \& Lightfoot, 1985). Multimodal programs are not limited to just one treatment approach and consist of a broad range of therapeutic modalities (Ross \& Lightfoot, 1985). The inclusion of several treatment approaches provides an environment to address substance abuse as well as a number of other potentially problematic areas. Many authors recognize the benefit of the multimodal philosophy for treatment (Gendreau \& Goggin, 1997; Goss Gilroy Inc., 1999; Lipton, 1998; McGuire \& Priestly, 1995; Pearson \& Lipton, 1999).

Multimodal substance abuse treatment can be described as holistic, because it not only attempts to address physical symptoms of abuse but also psychological and social factors that can precipitate substance abuse. Glider and colleagues (1997) reiterate the importance of this approach advocating, "the complex interaction between causal and correlational factors must be considered in the development and implementation of treatment programs for incarcerated offenders" (p. 97). Another important feature of the multimodal treatment approach is that it exposes participants to a variety of treatment modes and styles. Exposure to an array of treatment modes can increase the likelihood that a participant will discover his or her preferred treatment style, which may be more effective in changing his or her substance-abusing behaviour. 
One of the most common multimodal treatments is the TC. The MDH substance abuse treatment program most closely resembles a therapeutic community. For this reason, the TC model is reviewed in detail below. The review commences with the historical context of the TC. Common treatment goals within substance abuse TCs are outlined, followed by a description of the four defining characteristics of the TC. Subsequently, the MDH substance abuse treatment program is outlined providing another example of a multimodal substance abuse treatment program.

\section{The Therapeutic Community}

In chapter 1 of this literature review, the TC treatment model was described as one example of the various substance abuse interventions that have been applied in correctional settings. The TC is a substance abuse program that incorporates several treatment modalities with an emphasis on group therapy (Murray, 1996). This type of treatment focuses on creating a learning environment using peers and staff as role models. These communities are long-term, residential programs running approximately 8 to 12 months (Graham \& Wexler, 1997; Lightfoot, 1997; Yablonsky, 1989).

The history of the TC can be traced back to the United Kingdom, post World War II. The TC model surfaced as a means of treating war veterans who had developed neurotic illnesses as a result of their war experience (Goss Gilroy Inc., 1999). Also around this time, the term "therapeutic community" was used to describe an innovative method of organizing psychiatric hospitals. Within psychiatric hospitals, the TC was implemented as a forum for both staff and patients to participate in the management of the community with the goal of resocializing patients into the larger society (Brook \& Whitehead, 1980). The British were resolute with the idea that social factors affected 
treatment outcome (Rossi \& Filstead, 1973) and therefore, harnessing the social setting to assist in the treatment of mentally ill patients was seen as a means for successful rehabilitation.

As an extension of this model, the TC was moved from the hospital setting into the community. The community-organized TC demonstrated that psychiatric patients could live together and function well in terms of decision-making and employment (Brook \& Whitehead, 1980). The TC has also been applied to other settings, including corrections. Currently in the United States, the TC is a commonly practiced intervention for substance-abusing offenders. In 1997, approximately 110 TCs designed for substanceabusing offenders were in operation in US state and federal institutions (Lipton, 1998).

In an address to the Ninth World Conference of Therapeutic Communities in 1985, Monsignor William O'Brien, then president of the World Federation of Therapeutic Communities (WFTC), described the TC model:

There is no doubt that the therapeutic community is one of the most potent treatment modalities in helping individuals achieve change. The elimination of the symptom, be it substance abuse or other disorders, is only part of the treatment and help. We achieve change as a result of meeting the needs of individuals by providing the necessary services. The greatest need is a sense of community, a sense of belonging, and this is what the therapeutic community provides as it effectively deals with the pains of modern man - loneliness and alienation. The key, then, is that the therapeutic community deals with root causes rather than merely treating symptoms. (Yablonsky, 1989, p. 39) 
This holistic human change perspective described by Monsignor O'Brien is not only a significant feature of the TC (Inciardi et al., 1997; Lipton, 1998; Nielsen, Scarpitti, \& Inciardi, 1996; Pearson \& Lipton, 1999), but also it is in keeping with the multimodal treatment philosophy. With the recognized need to treat the whole person, as opposed to just the symptoms of abuse, the general goal of the therapeutic community then, is to create a healthy environment that allows individuals to grow morally and socially, and to become more competent (Brook \& Whitehead, 1980; Graham \& Wexler, 1997). In TCs designed for substance-abusing offenders, specific treatment goals can vary slightly depending on the treatment modalities incorporated into the program. However, a review of the literature reveals four common goals: (1) to achieve a substance-free status, (2) to terminate illicit and criminal behaviours, (3) to re-enter society through gainful employment or continuing education, and (4) to maintain stable and positive relationships (Brook \& Whitehead, 1980; De Leon, 1995; Goss Gilroy Inc., 1999; Nielsen et al., 1996). In an effort to fulfil the specific treatment goals outlined above, four defining characteristics are integrated into the substance abuse treatment TC: (1) the social structure of the TC is designed to ensure offenders are actively involved in the administration and maintenance of the TC; (2) offenders who have successfully maintained a substance-free lifestyle are used as "co-therapists"; (3) groups are used heavily; and (4) multiple treatment modalities are included (Goss Gilroy Inc., 1999; Graham \& Wexler, 1997; Lightfoot, 1997; Lipton, 1998; Yablonsky, 1989). These four defining characteristics of the TC are described in more detail below.

The social structure of the TC is designed to engage offenders in activities that stretch well beyond the treatment aspects of the therapeutic community, such as activities 
related to the administration and maintenance of the program. By involving offenders in the operations of the treatment program they are participating in, the TC does not adhere to the traditional doctor and patient dichotomy (Lipton, 1998; Pearson \& Lipton, 1999; Wexler, 1995; Yablonsky, 1989). This breadth of client involvement allows for upward mobility within the TC structure. Upward mobility is perceived as a positive influence for behaviour and attitude change, and a forum for teaching offenders about personal responsibility and employment (Wexler, 1995). As a result of this treatment structure, all offenders have work positions within the TC and they are encouraged to advance to higher levels of responsibility (Pearson \& Lipton, 1999).

In the hierarchical structure of the TC, the uppermost position an offender can attain is that of a "co-therapist" (Graham \& Wexler, 1997; Rossi \& Filstead, 1973; Yablonsky, 1989). Only those offenders who achieve a substance-free lifestyle are able to assume this position. As a "co-therapist", the offender assists with changing the substance-abusing behaviour of other offenders (Lightfoot, 1997; Rossi \& Filstead, 1973; Yablonsky, 1989). The ex-substance abuser is deemed an important component of treatment for a couple of reasons. First, ex-substance abusers in a TC have first-hand experience with the problem. They understand the pleasure and pain of using substances, they are familiar with the rationalizations and self-deceptions that abusers use, and they comprehend the struggle many offenders face trying to change their problematic behaviour. Second, ex-substance abusers within the TC are viewed as role models for other offenders coping with substance abuse. In the position of a 'co-therapist", the offender is an image of success providing others with hope that they too can change. 
Another vehicle of change within the TC is the group (Brook \& Whitehead, 1980; Gilder et al., 1997; Goss Gilroy Inc., 1999; Graham \& Wexler, 1997; Lipton, 1998; Yablonsky, 1989). Group mechanisms can be housed within the TC either to act as a positive and/or negative reinforcement for behaviour and attitudes or to increase language capacity and self-expression (Lipton, 1998; Pearson \& Lipton, 1999; Yablonsky, 1989). The "encounter group" is an example of a group structure used in most TC programs. It is believed that this group environment assists with changing substance-abusing behaviour and attitudes (Lipton, 1998; Pearson \& Lipton, 1999; Wexler, 1995). Encounter groups are usually run two to three times a week requiring participation of all offenders (Wexler, 1995). This group is used as a forum for offenders to explore their personal problems and commitment to be substance free. They are encouraged to discuss their progress as well as any work and organizational problems they may be experiencing. During an encounter group, offenders are also supported to provide feedback to others on their progress, behaviour, and attitudes. This feedback can be both positive and negative, and usually adheres to respectful parameters (Glider et al., 1997; Graham \& Wexler, 1997; Lipton, 1998; Yablonsky, 1989).

The fourth defining characteristic of the $\mathrm{TC}$ is that it incorporates diverse treatment modalities. Although the selection of treatment modalities tends to vary from TC to TC, all TCs consist of an array of treatment activities. Examples of modalities that can be included in a TC are: (1) group sessions (i.e., encounter groups and/or discussion groups), (2) one-on-one counselling, (3) drug and alcohol education, (4) relapse prevention, (5) AA, (6) family counselling, (7) academic opportunity, (8) psychodrama, and (9) video playback (Glider et al., 1997; Hartmann, Wolk, Johnston, \& Colyer, 1997; 
Lipton, 1998; Yablonsky, 1989). This programming structure is illustrative of the holistic nature of the $\mathrm{TC}$, and its commitment to treating the whole person.

Within the United States the therapeutic community is one of the most widely used multimodal treatment programs for substance-abusing offenders, especially within the community. However, correctional community substance abuse treatment programs exist that adhere to the multimodal philosophy without the incorporation of the other defining characteristics of the TC. The MDH substance abuse treatment program is an example of such a program. The next section of this chapter describes the MDH program, followed by a review of the program design and theory. The specific treatment modalities incorporated into the program are detailed as well as the program goals. This chapter concludes by highlighting the similarities and differences between the MDH program and the TC treatment model.

\section{MDH Substance Abuse Treatment Program}

Program description. The MDH substance abuse program is a residential substance abuse intervention for male federal offenders located in a half-way house in Ottawa, Ontario. This substance abuse program is described as a medium to high intensity treatment intended for offenders who are medium to high risk and have moderate to severe substance abuse problems. It should be noted that offenders participating in the MDH program are not diagnosed with a substance use disorder according to the DSM-IV criteria. Other substance abuse assessments are utilized by $\mathrm{MDH}$ and the CSC to determine whether an offender has a substance abuse problem, such as the ADS and DAST. However, according to the DSM-IV, offenders involved in the MDH program could be most accurately classified with substance abuse disorder, 
given that MDH does not systematically assess offenders on physiological symptoms of drug and alcohol use.

$\mathrm{MDH}$ is the only residential substance abuse program for male federal offenders and parolees in the Ottawa area. The substance abuse program delivered at MDH has been in operation since March 2, 1998. Upon recognition of the need for male federal offenders with substance abuse problems to be treated in the community, a substance abuse program was developed and the CSC was approached by MDH for funding. Given that the clients of the MDH program are male federal offenders falling under the jurisdiction of the CSC, MDH follows the same Mission as that of the CSC. This Mission states that "as part of the criminal justice system, (the CSC) contributes to the protection of society by actively encouraging and assisting offenders to become law abiding citizens while exercising reasonable, safe, secure, and humane control" (Correctional Service of Canada, 1998a, p. 4).

The program offered by MDH is structured in a half-way house and is supervised twenty-four hours a day, seven days a week by various staff. Twelve staff members are employed at the half-way house, headed by the Executive Director. Three of the twelve staff members are program staff, who deliver the program and are counsellors for the offenders. The program staff members are social workers ranging from the Bachelor to the Masters level. All program staff are required to have extensive experience in addictions and counselling. The other staff members are administrative staff, night shift staff, security staff, and the cook.

Participation in this program is usually an element of the offender's release plan and a condition of their release. Since program inception in 1998, an average of 70 
offenders participate in the MDH program annually, with an average annual program completion rate of $53 \%$. The yearly program completion rate has increased over the years, reaching a high of $58.5 \%$ in 2003 .

Program design and program theory. The MDH program design is similar to the $\mathrm{TC}$ in that it consists of several treatment modalities or activities. The evaluation literature describes program activities as having a direct impact on program targets, and suggests that these activities are intended to lead to the attainment of program goals (Rush \& Ogborne, 1991). The three program activities within MDH are: (1) counselling, (2) drug and alcohol education, and (3) cognitive-behavioural intervention. The MDH program anticipates a chain of events stemming from these program activities. These expected outcomes are part of the program theory or program logic, which can be represented in the pictorial format of a logic model (see Appendix A). ${ }^{11}$

The main purpose of the logic model is to demonstrate how specific program activities, outputs, and the various outcomes of the MDH program relate to each other. The logic model is an important evaluative tool, because it helps to explain the ideas behind the design of the program and the reasons the program is expected to elicit change (Treasury Board Secretariat, 2001). In the next three subsections of this chapter, each activity is described along with its supporting program theory.

11 This logic model is an adapted version of the original logic model (see Appendix B) representing the $\mathrm{MDH}$ program. This revised logic model was created to reflect the program theory that will be examined in the present evaluation, given that the original model incorporated outcomes beyond the current scope. The differences between the two models are: (1) the ultimate outcome of lifelong abstinence was removed, due to the fact that the scope of this evaluation is not lifelong; (2) the short-term and long-term outcomes related to personal goals are not being examined because of the complexity of addressing the various personal goals selected among participants; and (3) the long-term goal of desire to be substance free is not included because this construct is similar to the motivation variable, which is being measured. Even with these modifications, the revised logic model adequately represents the fundamental theory of the MDH program. 
Counselling activity. The counselling component of the program was included to provide participants with the opportunity to engage in one-on-one treatment. At intake to the program, each offender is assigned a counsellor. The first three to five counselling sessions have a structured format consisting of two elements. First, this initial counselling component is used to complete the intake assessment on the offender, and review the results with the offender. Second, during the initial counselling component, the counsellor must use motivational interviewing techniques.

The initial counselling activity is designed for the intake process and review of the intake results, so that the program counsellor and offender have an understanding of the offender's strengths and weaknesses with respect to substance abuse and other areas in his life. All subsequent counselling sessions the offender attends after the initial counselling activity are used to address his identified deficits. The motivational interviewing techniques were incorporated into the program design in an effort to increase an offender's motivation to participate in treatment and to move the offender through the stages of change.

It should be noted that MDH underwent an independent program review in early 2003 and recommendations for program modification were provided to MDH. Consequently, minor changes to the program design were made. One of the modifications was adding a few counselling sessions to the counselling activity. Originally, the counselling activity required program staff to use motivational interviewing techniques in the first counselling session. In mid October 2003, the use of motivational interviewing techniques in the first three to five counselling sessions was made mandatory for program staff. 
With respect to the initial counselling component and the incorporation of motivational interviewing techniques, this particular aspect of the program design is based on the transtheoretical model of readiness to change (DiClemente \& Velasquez, 2002; Prochaska \& DiClemente, 1986; Prochaska, DiClemente, \& Norcross, 1992). Although this model was originally conceptualized through the study of smoking behaviour, its application to other addictive behaviours, including substance abuse, has been recognized by its creators (Prochaska et al., 1992). The transtheoretical model suggests that behaviour change is a process, and those individuals who either are changing their behaviour on their own or participating in a program, progress through five stages of change (DiClemente \& Velasquez, 2002; Prochaska \& DiClemente, 1986). The stages of change represent points in time when an individual experiences shifts in attitudes, intentions, and behaviours (Prochaska et al., 1992). The five stages are: (1) precontemplation, (2) contemplation, (3) preparation, (4) action, and (5) maintenance.

Briefly, individuals in the precontemplation stage have not yet considered change, because they do not recognize that they have a substance abuse problem (DiClemente, 2003; DiClemente \& Velasquez, 2002; Prochaska et al., 1992; Soden \& Murray, 1997). In the contemplation stage, individuals are aware they have problem with substances; however, they have not taken steps towards change (DiClemente \& Velasquez, 2002; Prochaska et al., 1992; Soden \& Murray, 1997). Individuals in the preparation stage are committed to changing their behaviour and they have developed a plan for changing the behaviour (DiClemente, 2003). The action stage is when an individual has implemented their plan and taken steps to bring about change in his or her behaviour (DiClemente \& Velasquez, 2002; Prochaska et al., 1992). This stage requires a considerable commitment 
of time and thus, individuals tend to be classified in the action stage for approximately six months before progressing on to the final stage (DiClemente \& Velasquez, 2002;

Prochaska et al., 1992). The final stage is maintenance, and it has been described as the stage in which people work to prevent relapse and sustain the gains achieved in the action stage (DiClemente, 2003; DiClemente \& Velasquez, 2002; Prochaska et al., 1992; Soden \& Murray, 1997).

Research on readiness to change has discovered that treatment outcomes vary according to pretreatment stage of change. For example, it appears that being in the preparation or action stage at the beginning of program increases the likelihood of individuals maintaining their changed behaviour in comparison to individuals who start treatment in the precontemplation or contemplation stage (Prochaska \& DiClemente, 1992). Prochaska and DiClementi (1986) have argued that the different stages of change require different therapeutic strategies, and empirical reports seem to support this contention. Indeed, skills-based programs tend to be most successful for individuals who start the program in the action stage (Heather, Rollnick, Bell, \& Richmond, 1996). Thus, when individuals enter treatment either in precontemplation, contemplation, or preparation, treatment should focus on increasing motivation (Heather et al., 1996).

A variety of broad definitions of motivation exist (Saunders, Wilkinson, \& Towers, 1996); however, in relation to stages of change, motivation can be operationalized as a fluctuating state of readiness to change a behaviour (Bell \& Rollnick, 1996). Under this lens, motivation is often seen as a prerequisite for treatment (Brown \& Miller, 1993; Miller, 1985), including treatment for offenders (Ginsburg, Mann, Rotgers, \& Weekes, 2002). An intervention strategy known as motivational interviewing can 
positively influenced motivation (Miller, 1983). The underlying theoretical framework for motivational interviewing is the transtheoretical model developed by Prochaska and DiClemente.

Motivational interviewing (MI) is described as a directive and client-centered approach to counselling that increases intrinsic motivation among clients to change their problematic behaviour (Miller \& Rollnick, 2002). Essentially, MI is a method used to prepare individuals for treatment by helping them recognize and act on a problem (Miller \& Rollnick, 2002; Zweben \& Zuckoff, 2002). In accordance with the terminology of the transtheoretical model, the intent of $\mathrm{MI}$ is to move an individual to the next stage of change and potentially through all remaining stages (DiClemente \& Velasquez, 2002; Harper \& Hardy, 2000). Given that people are at different stages of change and that they can move back and forth between stages throughout the change process, $\mathrm{MI}$ is beneficial for individuals in all five stages of change (Bell \& Rollnick, 1996).

Research on this technique has demonstrated its efficacy with alcohol and drug abusers (Burke, Arkowitz, \& Dunn, 2002). Specifically, MI used with drug abusers has been documented as increasing treatment adherence and treatment completion rates, reducing use, prolonging relapse, and strengthening commitment to abstinence (Daley, Salloum, Zuckoff, Kirisci, \& Thase, 1998; Saunders, Wilkinson, \& Phillips, 1995; Stephens, Roffman, \& Curtin, 2000). With alcohol abusers, MI research has demonstrated reduced drinking rates and increased treatment participation (Brown \& Miller, 1993; Marlatt et al., 1998; Sellman, Sullivan, Dore, Adamson, \& MacEwan, 2001). 
The MI approach has been used with offender populations, and some research on its effectiveness has been undertaken. In a study with offenders of domestic violence, MI was effective in increasing motivation to take steps to change substance-abusing behaviour (Easton, Swan, \& Sinha, 2000). Harper and Hardy (2000) found positive attitude changes among substance-abusing offenders who were supervised by probation officers trained in MI. Relative to control participants, alcohol-dependent offenders within the Canadian federal system who received a motivational interview showed increased problem recognition, and precontemplators demonstrated more drinking behaviours consistent with the contemplation stage of change (Ginsburg, 2000). In a study of drug-abusing male offenders within the Canadian federal system, offenders who participated in a motivational interviewing session had a significant increase in their action scores a week later in comparison to two control groups (Vanderburg, 2002).

Drug and alcohol education activity. The drug and alcohol education component of the MDH program is used to educate participants on various issues related to substance abuse. The education activity is delivered as videos. Offenders watch the instructional videos shortly after program intake is completed. Each offender watches several videos about substance abuse, four of which are mandatory to view. A variety of topics are covered in the mandatory videos including: denial, anger, shame, and addiction. It should be noted that the drug and alcohol education activity was removed from the MDH program at the beginning of September 2003, in response to the recommendations from the program review. As a result, data on the effectiveness of the drug and alcohol education component could only be collected for 6 months. 
Drug and alcohol education programs have varying formats. Some of the more recognizable approaches are information dissemination, fear arousal, and moral appeals (Botvin, 1990). ${ }^{12}$ All of the approaches to education are based on a distinct model although the approaches may be combined into a single education program. The most commonly used method is information dissemination (Botvin, 1990), which is the approach taken by MDH. A drug and alcohol education program using an information dissemination approach can impart information in a variety of ways, including lectures, discussions, videos, pamphlets, posters, and public service announcements (Botvin, 1990; Correctional Service of Canada, 1991, 1998b; Miller \& Hester, 1986).

The underlying assumption of the information dissemination model is that substance abusers lack knowledge about substances and the possible consequences of substance use (Botvin, 1990; Correctional Service of Canada, 1991, 1996a, 1998b; Lipton et al., 1992; Miller \& Hester, 1986; Montague \& Scott, 1993). Therefore, education programs are delivered to increase knowledge among substance abusers thereby changing their attitudes about substance use and ultimately causing them to decrease their consumption (Botvin, 1990; Correctional Service of Canada, 1991, 1996a, 1998b; Lipton et al., 1992; Miller \& Hester, 1986; Montague \& Scott, 1993). Although this rationale is the foundation of information dissemination programs, the treatment model has been criticized for not evolving from theory and empirical evidence (Baldwin et al. 1991a; Botvin, 1990; Miller \& Hester, 1986). The literature is not only in dispute on whether increased knowledge of substances can actually change attitudes and behaviour (Baldwin, 1990; Correctional Service of Canada, 1991), but also on whether substance

12 Botvin (1990) provides a more in-depth description of these approaches and others. 
abusers do in fact lack knowledge about substances and problems associated with use (Correctional Service of Canada, 1996a; Lipton et al., 1992).

Regardless of the disagreement, several recommendations have been put forth concerning the implementation of drug and alcohol education programs (Correctional Service of Canada, 1991, 1996a, 1998b; Lipton et al., 1992). First, as a stand-alone program, education should be reserved for individuals with either minimal substance abuse problems or no substance abuse problems. This suggestion is based on the belief that individuals with minimal or no substance abuse problems have little knowledge of the effects of substances given their limited involvement with them and thus, they are more likely to benefit from an education program designed to increase knowledge of substances. Second, education programs should be a component of a multimodal treatment approach for more severe substance abusers. Specifically, education programs should be integrated with skills training to assist with behaviour change.

A cursory review of the outcome research conducted on drug and alcohol education programs seems to indicate conflicting results; that is, some studies conclude education programs work and others infer they do not. An examination of the dependent variables measured in the research reveals a source for the seemingly inconsistent results. Specifically, efficacy research varies on the selection of outcomes, which include: knowledge gain, attitudes, substance use, and recidivism. Thus, the effectiveness of education programs seems to be dependent on the outcome being measured (Baldwin, 1990; CSC, 1996a).

Another source for the differing results could stem from the inadequate or nonexistent program descriptions provided in the research studies. It has been noted in 
the literature that often researchers do not provide a description of the education program under review (Baldwin, 1990). Without this detail, it is difficult, if not impossible, to determine what education approach is being investigated. As a result, not only could differing programs be inappropriately compared, but also little is known about the efficacy of the various educational approaches.

Nonetheless, consistently positive results have been achieved when knowledge, and to a lesser extent, attitudes were examined (Botvin, 1990). A study by Sean (as cited in Correctional Service of Canada, 1996a) found a significant increase in drug and alcohol knowledge and a significant reduction in drug and alcohol use attitudes among individuals who participated in a substance abuse education course as an aftercare component to treatment. The sample consisted of former abusers, half of whom had been incarcerated. Findings from a study of probationers participating in an alcohol education program revealed higher scores on an alcohol knowledge test after the program in comparison to their preprogram scores (Baldwin, 1990). Further evidence of changes in knowledge were revealed in a comparative evaluation. In a study by Stalonas, Keane, and Foy (1979), participants who watched education presentations on videotape had significantly greater knowledge gain in comparison to participants who either attended lectures or read written material on the videos (as cited in Miller \& Hester, 1986).

When substance use is the dependent variable, varying results emerge. In a controlled study of a prerelease alcohol education program for young offenders, Baldwin and his associates (1991b) found reduced drinking at a follow-up of at least 9 months for the treatment group in comparison to the control group. In contrast, earlier studies with offender samples, which were randomly assigned, found no differences in alcohol 
consumption rates between offenders who participated in the education program and offenders who did not (Scoles \& Fine, 1977; Swenson \& Clay, 1980).

Research on the effectiveness of drug and alcohol education programs in reducing recidivism seems to hold promise. Baldwin and his colleagues (1991b) described positive findings in their study. Young offenders who participated in an alcohol education program reported less criminal activity than the control group. In another study conducted by Baldwin and his associates (1991a), they examined the impact of alcohol education programs on court-referred young offenders. Although no differences between the treatment and control group were found for police-reported offending rates, a withingroup comparison revealed a reduced number of recorded offences after the education program than before. In a study of 331 individuals convicted of impaired driving, Jamieson and Stone (1991) found a small percentage of impaired drivers who completed an education program were rearrested two years after program completion $(10 \%)$. In addition, a meta-analysis concluded from an examination of the education literature that this type of program showed potential for reducing future criminal behaviour, and they advocated for further examination because of the small number of studies included in the analysis (Pearson \& Lipton, 1999).

Cognitive-behavioural intervention activity. The cognitive-behavioural activity is approximately 140 hours in length and delivered five days a week. Offenders participate in 1 two-hour session each morning and 1 two-hour session each afternoon over about a 7-week period. The sessions are intended to increase knowledge and ability in two specific skill areas - prosocial and relapse prevention. Approximately 35 hours of this 
program activity are spent on prosocial skills and another 35 hours are dedicated to relapse prevention skills.

Due to the 2003 program review, the content of the cognitive-behavioural activity was reorganized slightly in mid October 2003. Specifically, all issues related to a topic were to be delivered consecutively and the material reviewed in its entirety, instead of mixing various topics together and not covering all issues related to the topic before introducing another topic. The delivery of the cognitive-behavioural activity is alternated daily among the three program staff. Content quizzes and program feedback forms are completed after many of the group sessions, and selected group sessions require participants to complete homework assignments.

Social learning theory forms the theoretical base for the cognitive-behavioural activity in the MDH program. The social learning perspective holds that behaviours are largely the result of past learning experiences. Indeed, social learning theory asserts that behaviour is learned and can be unlearned or controlled through the development or enhancement of cognitive and behavioural skills (Bandura, 1977). Thus, substance abuse is viewed as a maladaptive behaviour mediated by cognition that can be changed through the process of learning (Botvin, 1990; Dobson \& Dozois, 2001; Marlatt, 1985).

Cognitive-behavioural treatment attempts to change a specific maladaptive behaviour by monitoring and altering cognitive activity. Consistent with this description of cognitive-behavioural treatment, the two primary outcomes of treatment are cognition and behaviour change (Dobson \& Dozois, 2001). Evaluations of the cognitivebehavioural substance abuse treatment programs implemented across Canadian federal institutions have demonstrated that participation in these programs can increase 
knowledge in several areas, including substance abuse consequences, responding to relapse situations, and problem-solving skills as well as reduce future criminal activity among offender populations ( $\mathrm{T}^{3}$ Associates Inc., 1999a, 1999b, 1999c; Millson et al., 1995). A meta-analysis conducted by Pearson, Lipton, Cleland, and Yee (2002) provides additional support for the efficacy of cognitive-behavioural treatment to reduce recidivism. Results revealed a mean effect size of $r=.14$. The corresponding BED is a recidivism rate of $43 \%$ for the experimental group versus a rate of $57 \%$ for the comparison group.

In McGuire's textbook on cognitive-behavioural treatment (as cited in Pearson et al., 2002), this intervention is viewed as a collection of methods or techniques versus a single method. McGuire includes the following techniques in the collection: social skills training, problem-solving training, cognitive skills programs, and the relapse prevention model. Similar to McGuire's collection of methods, Rotgers (1996) believes a number of essential elements of cognitive-behavioural intervention should be used with substanceabusing offenders. Two of the core elements are skills training and relapse prevention. As recommended by Rotgers (1996), the cognitive-behavioural component of the MDH program consists of skills training and relapse prevention. ${ }^{13}$

In general, the literature describes skills training as a means for providing clients with skills to cope more effectively in problematic social situations (Morgan, 1996). Among substance abusers, it appears that they have inadequate skills to respond to a variety of social situations, which can influence use and relapse. Some of the skills this population can be deficit in are: problem-solving, assertiveness, and anger management

13 In the MDH program, skills training is referred to as prosocial skills training. 
(Hawkins, Catalano, \& Wells, 1986; Miller \& Hester, 1986; Morgan, 1996; Vigdal, Stadler, Goddrick, \& Stutton, 1980).

Studies using an experimental design provide evidence of the effectiveness of skills training with nonoffender substance-abusing samples. Specifically, after involvement in this type of treatment, participants' performance improved in situations involving avoidance of substance use and problem-solving (Chaney, 1989; Hawkins et al., 1986). In addition, the meta-analysis by Pearson et al. (2002) supports the use of skills training with offender populations to reduce recidivism. Based on 14 primary research studies, a mean effect size of $r=.17$ was found, which corresponds to a BED of $41.5 \%$ recidivism in the treatment group compared to $58.5 \%$ in the comparison group.

In the MDH program, prosocial skills are presented to the offenders in two ways. First, for a variety of life situations the offender encounters on a daily basis, participants are introduced to more appropriate and effective thought and behaviour responses. Examples of these life situations are anger and cravings. Second, program staff teach participants techniques to improve their assertiveness and problem-solving skills.

The relapse prevention model was developed as a treatment technique to prevent relapse. Marlatt (1985) defined relapse as "a breakdown or setback in a person's attempt to change or modify any target behaviour" (p.3), including substance abuse behaviour. When incorporated into a substance abuse treatment program, relapse prevention seeks not only to deter the initial return to substance use, but also continued usage if an individual starts to use again (Donovan, 2003). Thus, the emphasis of relapse prevention is to teach individuals how to identify high-risk situations and how to cope with these 
situations when they occur (Dimeff \& Marlatt, 1998; Donovan, 2003; Marlatt, 1985; Parks, Marlatt, Young, \& Johnson, 2003).

While formulating the relapse prevention model, Marlatt identified eight high-risk situations that precipitate relapse (Annis, Sklar, \& Turner, 1997; Marlatt, 1985): (1) unpleasant emotions, (2) physical discomfort, (3) conflict with others, (4) pleasant times with others, (5) pleasant emotions, (6) urges/temptations, (7) social pressure to use, and (8) testing personal control. In addition, Marlatt believed that self-efficacy ratings were the most significant self-report measure of ability to identify and cope with high-risk situations. Thus, subjective confidence scores could be used as a measure of coping capacity as well as a method for identifying potential high-risk situations. When identifying personal high-risk situations, lower self-efficacy ratings for any of the eight situations would highlight areas that tend to encourage use.

Relapse prevention appears to be an effective method of treatment for substance abuse. Indeed, the meta-analysis conducted by Irvin et al. (1999) of the literature on the efficacy of relapse prevention with nonoffender samples found a mean effect size of $r=$ .14 for reduced substance use. Not many studies have been conducted with substanceabusing offenders. However, a study by Peters and colleagues (1993) provides preliminary support for the effectiveness of relapse prevention with a criminal population. This study found that offenders who participated in a cognitive-behavioural treatment focusing on relapse prevention increased their skills in responding to high-risk situations and increased their repertoire of knowledge related to relapse.

MDH incorporates relapse prevention into the cognitive-behavioural component of the program by training participants how to think and behave when trying to avoid 
relapse to substance use. The concepts of relapse prevention and the identification of high-risk situations are examined with participants. After an introduction to these various topics and skills, participants develop their own relapse prevention plan outlining their personal high-risk situations and areas in their lives requiring more prosocial skill. The relapse prevention plan also documents the appropriate thought and behaviour responses for their personal high-risk situations. During group, offenders engage in role-playing exercises to practice identifying high-risk situations and to practice using their personal relapse prevention plan. Program participants are also encouraged to practice their new skills outside of the cognitive-behavioural activity.

As can be seen in Appendix A, the three program activities generate program outputs. Program outputs are the link between the program activities and the short-term program outcomes (Rush \& Ogborne, 1991). More specifically, outputs are the goods and services of the program delivered to the participants providing evidence that the activity occurred (Treasury Board Secretariat, 2001). It is important to be aware that program outputs are not the same as program outcomes. Unlike outputs, which demonstrate that an activity has taken place, outcomes are the results of a specific program activity (Rossi et al., 1999). Each activity in the MDH program has two to four outputs.

Generated from the counselling activity are two outputs. The first output resulting from the counselling component is the notes kept by the counsellor. Program staff are required to keep notes on each session outlining the issues covered in the session, the progress of the offender, and any concerns the program staff might have about the offender. The second output is the completed Readiness to Change Questionnaire Treatment Version (RCQ-TV; Heather, Luce, Peck, Dunbar, \& James, 1999). 
The educational component of the MDH program produces three outputs: (1) the completed prevideo questionnaires, (2) the completed postvideo questionnaires, and (3) the number of video showings. Four outputs are generated from the cognitive-behavioural intervention activity: (1) the number of cognitive-behavioural sessions available for the offender to participate in, (2) the number of sessions attended by a particular offender, (3) the number of homework assignments complementing this activity, and (4) the number of assignments completed by the offender.

Short-term outcomes are also presented in a logic model. Short-term outcomes can be described as changes in participants (offenders) as a result of their participation in an activity with the change occurring soon after the activity is performed (Rossi et al., 1999; Treasury Board Secretariat, 2001). With completion of each program activity, the MDH program hopes to achieve several specific short-term outcomes (see Appendix A). The first, stemming from the counselling activity, is increased motivation for treatment and increased motivation to change substance-abusing behaviour. By participating in the drug and alcohol education component, the program seeks to increase knowledge about substance abuse. The short-term outcomes from the cognitive-behavioural activity are increased knowledge of prosocial skills and increased knowledge of relapse prevention skills.

In addition to short-term outcomes, a logic model depicts long-term outcomes. Long-term outcomes are changes in the participants (offenders) as a result of their participation in an activity that occur after the short-term outcomes have been achieved. With the completion of the three program activities, the MDH substance abuse treatment program hopes to achieve several long-term outcomes (see Appendix A). One of the 
long-term outcomes is increased confidence to not use substances. The other long-term outcomes of the program are increased ability with prosocial skills and increased ability with relapse prevention skills. These two outcomes require that participants have the ability to use prosocial skills, such as problem-solving and assertiveness, as well as relapse prevention techniques, including the identification of high-risk situations.

Differences and similarities between $M D H$ and the TC. From the program description, design, and theory presented above, it becomes apparent that the MDH program does not adhere to two of the four defining TC characteristics. First, MDH does not recruit ex-substance abusers from the program to assist in the treatment of other offenders. Second, MDH's treatment structure more closely resembles that of the doctor and patient dichotomy. Offenders are expected to only participate in their treatment, which is delivered solely by program staff. Offenders are not involved in the administration of the program. Given this dichotomy, offenders are not accountable for any program responsibilities. Nonetheless, offenders participating in the MDH program are tasked with the overall maintenance of the half-way house. The rationale for this engagement of participants is similar to the $\mathrm{TC}$ in the sense that it is viewed as a mechanism for teaching offenders about personal responsibility. However, these general labour responsibilities are not part of a hierarchical structure as in the $\mathrm{TC}$, and are not used as a mechanism for strengthening employment values.

Similar to the TC, MDH is guided by the multimodal philosophy. MDH incorporates three specific treatment modalities or activities (i.e., counselling, drug and alcohol education and cognitive-behavioural intervention) within its program structure. In addition, MDH emulates the TC in its use of group sessions. Within $\mathrm{MDH}$, group 
sessions are used to facilitate critical aspects of the program targeting behaviour and attitude change. Another commonality between these two programs, beyond the defining characteristics, is the implicit use of some of the principles of effective correctional treatment, such as targeting offenders with the criminogenic need of substance abuse and applying the multimodal approach to substance abuse treatment. Given the limited acknowledgment of these principles within both program structures, the next chapter of this literature review outlines these empirically established correctional programming guidelines. 
Chapter 3: The Principles of Effective Correctional Treatment

A major force behind the anti-rehabilitation movement of the 1970 s was the research completed by Robert Martinson. Martinson (1974) examined treatment studies completed worldwide between the years of 1945 and 1967, and he concluded, "the rehabilitative efforts that have been reported so far have had no appreciable effect on recidivism" (p. 25). Although corroboration of this conclusion was minimal, the notion that "nothing works" was widely accepted. To challenge this doctrine, other researchers explored the conditions under which offender treatment programs are effective (Andrews et al., 1990; Gendreau \& Ross, 1980; Lipsey, 1995).

Using the psychology of criminal conduct as well as prior studies on the effectiveness of correctional treatment as a foundation, ${ }^{14}$ Andrews, Zinger et al. (1990) conceptualized appropriate correctional treatment as containing the principles of risk, need, and responsivity. Moreover, these researchers hypothesized that appropriate correctional treatment would reduce recidivism. In a flagship meta-analysis, this hypothesis was tested. Eighty-nine treatment studies were classified into four distinct types of treatment: (1) criminal sanctions, (2) inappropriate treatment, (3) appropriate treatment, and (4) unspecified correctional service, and their rates of recidivism compared. From this examination Andrews, Zinger and colleagues concluded, "the major source of variation in effects on recidivism was the extent to which service was appropriate according to the principles of risk, need, and responsivity" (p. 384). More

14 The psychology of criminal conduct (PCC) strives to understand the variation in the occurrence of criminal activity as well as the individual differences in criminal behaviour (Andrews \& Bonta, 2003). A more in-depth discussion of the psychology of criminal conduct can be found in the text by Andrews and Bonta (2003) entitled The Psychology of Criminal Conduct. 
recent meta-analyses continue to support the efficacy of the principles of effective correctional treatment (Dowden, 1998; Dowden \& Andrews, 1999; Lipsey, 1995; Lösel, 1995).

In chapter 1 , the principles of risk, need, and responsivity were briefly described. Now, a more in-depth discussion of these principles is presented. Each principle is defined and empirical research and/or literature supporting the principle is reviewed.

\section{Risk Principle}

The principle of risk encompasses two related elements: (1) risk can be predicted, and (2) risk should be matched to treatment intensity. The first element of the risk principle is that future criminal behaviour can be predicted. Risk factors, which are variables related to criminal behaviour, are measured to predict an offender's risk for reoffending. Two types of risk factors exist - static and dynamic. Static risk factors are historical variables found to be associated with criminal behaviour that do not change or when targeted through intervention cannot be changed, for instance, previous convictions or employment history. Dynamic risk factors are also related to criminal behaviour. However, these factors can fluctuate or be targeted to change with intervention. Examples of dynamic risk factors are substance abuse and antisocial associates.

Within the last few years, two meta-analyses examining the predictors of general and violent reoffending among adult offenders have been conducted (Bonta, Law, \& Hanson, 1998; Gendreau, Goggin, \& Little 1996). These meta-analyses explored the association between static and dynamic risk factors and future criminal behaviour, primarily among male offender samples from both a mentally disordered offender population and the general offender population. 
Gendreau et al. (1996) attempted to ascertain which risk factors were the best predictors of general adult recidivism. These researchers completed a literature search for relevant studies published between 1970 and 1994. After applying specific selection criteria, 131 studies were included in the meta-analysis. Within all of these studies, recidivism was defined as either an arrest, conviction, incarceration, parole violation, or a combination thereof.

Results indicated that static factors, such as adult criminal history $(r=.17)$, family factors (i.e., family rearing practices; $r=.14)$, and age $(r=.11)$ as well as dynamic factors, such as antisocial associates $(r=.21)$, antisocial personality $(r=.18)$, social achievement (i.e., being single, low levels of education, and poor employment history; $r$ $=.13)$, and substance abuse $(r=.10)$ were significant predictors of recidivism. Weak predictors of future criminal behaviour were identified as socioeconomic status $(r=.05)$, personal distress $(r=.05)$, and intellectual functioning $(r=.07)$.

In 1998, a meta-analysis by Bonta et al. was completed. These researchers examined the prediction of general and violent recidivism among mentally disordered offenders and compared the results to predictors identified for the general male criminal population. Applicable literature was gathered dating from 1959 to 1995 . Fifty-eight studies met the selection criteria. General recidivism was operationalized as any new offence, such as arrests and convictions as well as a recommitment to a psychiatric hospital because of law breaking behaviour. Violent recidivism was restricted to criminal reoffending of a violent nature.

For general recidivism, the static risk factor of criminal history, including adult criminal behaviour $(r=.23)$ and juvenile delinquency $(r=.24)$, proved to be a potent 
predictor of future criminal behaviour. Another significant static predictor was gender (i.e., being male; $r=.11$ ). Dynamic risk predictors significantly associated with general recidivism were antisocial personality $(r=.15)$, poor living arrangements $(r=.12)$, substance abuse $(r=.11)$, and family problems $(r=.10)$. These findings are similar for violent reoffending with one notable difference. Criminal history variables were not powerful predictors of violent recidivism; yet, when the criminal history variables were more specific to violent behaviour (i.e., violent history, violent index, and offence seriousness), the effect sizes increased.

With the recognized predictive ability of static and dynamic risk factors, actuarialbased risk assessments have been developed to assist with offender management. In relation to general recidivism, three distinct generations of risk assessment have been described. The third-generation of risk assessment is deemed the most versatile risk assessment because it incorporates both static and dynamic risk factors (Andrews \& Bonta, 1998, 2003; Bonta, 1996). ${ }^{15}$

Meta-analytic results clearly illustrate that risk to reoffend can be predicted. Furthermore, research supports the use of static and dynamic risk factors in risk assessment. Stemming from this first element of the risk principle is the second element

15 The first-generation of risk assessment involves a clinical professional making a personal judgment on the likelihood of an offender reoffending upon release (Bonta, 1996; Monahan \& Steadman, 1994). The second-generation of assessment represents an improvement over the first because it is actuarial-based. This type of assessment has explicit criteria for making decisions that are validated by research, facilitating the replication of risk decisions (Bonta, 1996; Monahan, 1996). Third-generation risk assessments are also actuarial-based. However, the main difference between the second-and third-generations is that the thirdgeneration includes dynamic risk factors (Bonta, 1996). Consequently, where the second-generation only incorporates static risk factors for the sole purpose of risk classification, the third-generation not only can measure an offender's risk but also changes in that risk level. Furthermore, the third-generation can be used to manage and possibly reduce risk through the offender's participation in treatment programs targeting individual dynamic risk factors identified by the assessment. 
of risk, which is that correctional treatment is most effective when it is matched to an offender's risk level (Andrews, 1989; Andrews \& Bonta, 1998, 2003; Farabee, Prendergast, Cartier, Wexler, Knight, \& Anglin, 1999; Taylor, 1998).

To match an offender to appropriate intervention based on his risk to reoffend, the offender's risk level is identified using an actuarial assessment, which allows for the classification of an offender into one of a range of risk levels (i.e., low, medium, or high). This assessed risk level is matched to the intensity of a treatment program. For example, according to the risk principle, offenders identified as high risk should be involved in intensive service. Research exploring the effects of treatment typically report treatment success when the treatment intensity is matched to the risk level of the offender (Andrews, Zinger et al., 1990; Bonta, 1996; Gendreau, 1996; Farabee et al., 1999; McGuire \& Priestley, 1995).

The risk principle underpins the assessment of risk and the matching of treatment, and alludes to appropriate targets for treatment. It is this latter use of the risk principle that is related to the second principle of effective correctional treatment - the need principle.

\section{Need Principle}

This principle states that to decrease criminal activity among offenders, need factors have to be targeted through intervention (Andrews \& Bonta, 1998, 2003). Specifically, two types of need factors have been identified: (1) criminogenic needs, and (2) noncriminogenic needs. Criminogenic needs are problems, circumstances, or characteristics that are known to promote criminal behaviour. Thus, when a criminogenic need is addressed through treatment, the change is associated with decreased recidivism 
(Andrews, 1995; Andrews \& Bonta, 1998, 2003; Andrews, Bonta et al., 1990). Some examples of criminogenic needs are substance abuse, antisocial attitudes, and antisocial associates. Noncriminogenic needs are also problems, circumstances, or characteristics that can be incorporated into correctional treatment. Although dynamic and changeable like criminogenic needs, noncriminogenic needs, when changed, are not associated with decreased reoffending (Andrews, 1995; Andrews \& Bonta, 1998, 2003; Andrews, Bonta et al., 1990). Self-esteem, socioeconomic status, and personal distress are examples of less promising treatment targets.

The need principle is related to the risk principle in the sense that criminogenic needs are dynamic risk factors (Andrews \& Bonta, 1998, 2003). Recall that dynamic risk factors are predictors of recidivism that can change over time. Dynamic risk factors are predictive of future criminal behaviour and are the criminogenic needs that when changed through intervention can decrease the probability of recidivism.

In the previous section of this chapter, various dynamic risk predictors were identified through risk prediction research. This risk prediction research not only identifies dynamic risk factors, but it also draws attention to potential criminogenic needs. Another body of research, albeit substantially smaller, has examined the ability of specific treatment programs to reduce future criminal behaviour (Andrews \& Bonta, 1998, 2003), highlighting promising treatment targets (i.e., criminogenic needs). Table 1 provides a synthesis of these information sources and outlines both promising and less promising treatment targets (i.e., criminogenic and noncriminogenic needs, respectively). 
Table 1

Promising and Less Promising Treatment Targets

\begin{tabular}{lll}
\hline \multicolumn{1}{c}{ Promising Targets } & \multicolumn{1}{c}{ Less Promising Targets } \\
\hline - Antisocial attitudes & - Self-esteem \\
- Antisocial feelings & - Cohesiveness of antisocial peers \\
- Family affection/communications & - Emotional/personal distress \\
- Self-control and management & - Socioeconomic status \\
- Substance abuse & $=$ Intellectual functioning \\
\hline
\end{tabular}

Note. Table is adapted from Andrews \& Bonta (1998). 
Responsivity Principle

Of the three principles of effective correctional treatment, the responsivity principle has had the least amount of research conducted to demonstrate its viability. Nonetheless, the responsivity principle is deemed integral to the success of correctional treatment (Andrews, 1989). This principle suggests that correctional treatment programs should be delivered in a manner consistent with the ability and learning style of offenders (Andrews, 1989; Andrews \& Bonta, 1998, 2003; Dowden \& Andrews, 1999; McGuire \& Priestley, 1995; Serin \& Kennedy, 1997). Two types of responsivity considerations contribute to this treatment principle: (1) general responsivity, and (2) specific responsivity.

The general responsivity principle draws upon the general personality and social psychology of crime to advocate appropriate modes of treatment for offenders. As a result, the cognitive-behavioural approach, which is a universal learning style for people in general, is considered to be the most appropriate method of treatment delivery for offenders (Andrews, 1995; Andrews \& Bonta, 1998, 2003; Gendreau, 1996; Gendreau \& Goggin, 1997; Lösel, 1995; Pearson et al., 2002). Other treatment methods, such as didactic instruction, psychodynamic therapy, and client-centered counselling seem to impede learning among most offenders (Andrews, 1989; Andrews, Bonta et al., 1990; Andrews, Zinger et al., 1990; Gendreau \& Goggin, 1997; McGuire \& Priestley, 1995). Thus, according to the general responsivity principle, a substance abuse treatment 
program based on the cognitive-behavioural philosophy is more apt to reduce recidivism than a substance abuse program that does not adhere to this principle. ${ }^{16}$

The specific responsivity principle concentrates on other individual characteristics of the offender, beyond learning style, that may influence the success of a treatment program, such as interpersonal sensitivity, interpersonal anxiety, verbal intelligence, cognitive maturity, and motivation (Andrews \& Bonta, 1998, 2003; Dowden \& Andrews, 1999; Gendreau, 1996; Serin \& Kennedy, 1997). In addition, the specific responsivity principle encourages matching client characteristics to caseworker/therapist characteristics (Andrews, Bonta et al., 1990; Gendreau, 1996; Gendreau \& Goggin, 1997).

Expansion of the Principles of Effective Correctional Treatment

The three principles of effective correctional treatment were intended to serve as a guide for correctional treatment development and implementation. Yet, since their inception, Dr. Don Andrews, the primary theorist, has seen merit in their expansion. Andrews (1995) sees utility in expansion because it provides "a more step-by-step set of principles" (p.41). Other criminal justice researchers have also advanced this personal vision (Gendreau \& Goggin, 1997; Gendreau, Goggin, \& Smith, 2001; McGuire \& Priestley, 1995; Lösel, 1995). A more comprehensive set of principles could serve to

16 Treatment has two dimensions: (1) a directive dimension, and (2) a relationship dimension (Andrews \& Bonta, 1998, 2003; J. Bonta, personal communication, September 20, 1999). Behaviourally-based treatment is primarily rooted in the directive (i.e., structured) dimension, providing direction to clients in his or her effort to deal with a particular problem. On the other hand, psychodynamic therapy and clientcentered counselling are clinical treatment modalities that emphasize the relationship dimension. These treatment environments are unstructured and self-teaching, providing clients with little guidance and/or direction. In addition, psychodynamic therapy and client-centered counselling tend to not address criminogenic needs. Much recidivism research indicates that these nonbehavioural treatment modalities do not perform as well as behavioural approaches (J. Bonta, personal communication, September 20, 1999; Lösel, 1995). 
further guide the development and implementation of correctional programming, and potentially result in the creation of better treatment services for offenders.

Of the extensions put forth to date, several have not been extensively researched (Andrews, 1995; Gendreau \& Goggin, 1997). Nonetheless, they are based on rational and empirical support (Andrews, 1995). Within the literature, some of the additional principles discussed most frequently are: (1) multimodal programming, (2) community programming, (3) continuity of care, (4) length of treatment, and (5) program integrity. ${ }^{17}$ Thus, a number of program characteristics appear to differentiate between more effective and less effective correctional treatment programs. Summaries of the five additional principles are provided later in this chapter.

Even though a panacea does not exist with the principles of effective correctional treatment (Gendreau, 1996; Lösel, 1995), researchers have operationalized appropriate treatment as integrating two to three of the original principles of effective correctional treatment (Andrews, Zinger et al., 1990; Dowden \& Andrews, 2004), and it has been assumed that the more principles that are incorporated into a program, the greater the chance it will reduce recidivism (McGuire, Broomfield, Robinson, \& Rowson, 1995).

\footnotetext{
17 It should be noted that some of these additional principles are components of the Correctional Program Assessment Inventory, the Correctional Program Accreditation Criteria used by the Correctional Service of Canada, and the Accreditation Criteria developed by Her Majesty's Prison Service. The Correctional Program Assessment Inventory (CPAI; Gendreau \& Goggin, 1997; Gendreau, Goggin, \& Smith, 2001) has been used to assess offender treatment programs on six dimensions related to the principles of effective correctional treatment. The Correctional Service of Canada developed an accreditation system for its programs, which stems from work done by the English and Scottish Prison Services $\left(\mathrm{T}^{3}\right.$ Associates Inc., 1997). This system involves an international panel of experts assessing a program with a predetermined set of evidence-based criteria based on the "what works" literature. Essentially, this external assessment establishes whether a program adheres to the principles of effective correctional treatment. To date, several institutional programs within the Service have been accredited, including OSAPP and CHOICES. Further information on these two accreditation processes can be found in: the Standards for Correctional Programs (Correctional Service of Canada, 2003), McGuire (2001), Lipton, Thornton, McGuire, Porporino, and Hollin (2000), and Porporino (1997).
} 
Hence, all of the aforementioned principles could be used as criteria for gauging the ability of a program to potentially influence recidivism. In addition, the criteria could serve as a method for assessing the appropriateness of the design and delivery of a program, which has been referred to as program implementation (Gendreau \& Goggin, 1997; Gendreau et al., 2001). Using the extended principles in this way would address an identified gap in the literature, given that few studies have been completed on the issue of program implementation (Gendreau et al., 2001).

Multimodal programming. Appropriate treatment should be multimodal (Gendreau \& Goggin, 1997; Goss Gilroy Inc., 1999; Lipsey, 1995; Lipton, 1998; Lösel, 1995; McGuire \& Priestly, 1995; Ross \& Lightfoot, 1985; Pearson \& Lipton, 1999). As discussed in chapter 2 , a multimodal program is not limited to just one treatment approach, but is comprised of a broad range of therapeutic modalities (Ross \& Lightfoot, 1985). The inclusion of several treatment approaches provides an environment to address a range of offender needs, particularly criminogenic needs. If an offender has multiple deficits that, in combination, increase the probability of recidivism, then all deficits need to be addressed (Gaes, 1998). In addition, multimodal programming creates a treatment forum in which the offender is exposed to an array of treatment styles, allowing him to take advantage of the style(s) most suitable for his rehabilitation.

Community programming. Community-based treatment services yield more positive effects than institutionally-based services (Andrews, 1995; Gaes, 1998; Gendreau, 1996; Lipsey, 1995; Lösel, 1995; McGuire \& Priestley, 1995). The greater ability of community programs to reduce recidivism in comparison to institutional programs does not negate the utility of rehabilitation in the institution. However, it does 
imply that programming situated closer to an offender's natural setting facilitates real-life learning (Gendreau, 1996; Gendreau \& Goggin, 1997; McGuire \& Priestley, 1995).

Continuity of care. Another additional principle of effective correctional treatment is the provision of aftercare (Gendreau \& Goggin, 1997; Ross \& Lightfoot, 1985; Wexler, 1994). Aftercare in a community-based program is usually delivered as a maintenance component to the formal treatment. Thus, maintenance is an adjunct to treatment, and an offender participates in these sessions once the formal portion of treatment is completed. Aftercare serves to sustain treatment effects, and provides an offender with the opportunity to deal with problematic situations that may arise outside of the treatment environment (Ross \& Lightfoot, 1985). Several core substance abuse programs within the Canadian federal correctional system apply maintenance to the treatment design (Correctional Service of Canada, 1996b; 1997).

Length of treatment. Another recurring principle of effective correctional treatment is the length of the treatment program. Research has demonstrated that a critical factor related to positive treatment outcomes (i.e., reduced recidivism and relapse) is the length of time spent in treatment (Inciardi et al., 1997; Wexler, 1995; Wexler, DeLeon, Thomas, Kressel, \& Peters, 1999). For intensive treatment services, both Gendreau (1996) and Lipsey (1995) promote at least 100 hours of treatment and indicate that the treatment should be delivered over a 3- to 9-month period. In addition, the literature warns that treatment spanning over one year may lead to diminishing results (Gendreau, 1996; Wexler, 1995).

Program integrity. Effective treatment must be implemented, supervised, and monitored well (Andrews, 1995; Gendreau, 1996; Gendreau \& Goggin, 1997; Lipsey, 
1995; McGuire \& Priestley, 1995; Wexler, 1994). To successfully implement a treatment program, program staff need to have specific characteristics and qualifications including: strong interpersonal skills, completion of an undergraduate degree or equivalent training in the theories of criminal behaviour and the prediction and treatment of criminal behaviour, and completion of 3 to 6 months of formal and on-the-job training (Andrews, 1995; Gendreau, 1996; Gendreau \& Goggin, 1997; Gendreau, Goggin, \& Smith, 1999; McGuire \& Priestley, 1995; Wexler, 1994). Well-supervised program staff also contributes to program effectiveness. Thus, the program design should incorporate quality assurance, in which program staff are assessed periodically on the quality of service delivery (Andrews, 1995; Gendreau, 1996). Program monitoring should involve continual assessment of adequate resources as well as the integration of an ongoing program evaluation plan to facilitate the fulfilment of program goals (McGuire \& Priestley, 1995; Wexler, 1994; Wexler, Blackmore, \& Lipton, 1991).

This chapter reviewed the three original principles of effective correctional treatment. Ample empirical evidence exists on the treatment effect programs have when the principles of risk, need, and responsivity are included. Five additional principles of effective correctional treatment supported by multiple researchers were summarized. Literature supports the inclusion of these five additional treatment principles in programming, when the goal is to influence behaviour and reduce recidivism.

Unfortunately, the TC model of treatment and the MDH substance abuse treatment program only implicitly recognize some of the principles of effective correctional treatment. Furthermore, within the TC literature, it appears as though no attempt has been made to examine their utility or to determine whether successful 
programs have incorporated any of these principles. The next chapter outlines the firstgeneration and second-generation substance abuse program evaluation literature. This chapter highlights the type of evaluations completed to date, and it will make the implicit incorporation and limited examination of these principles apparent. 
Chapter 4: First- and Second-Generation Substance Abuse Program Evaluations

Using the first- and second-generation evaluation taxonomy, this chapter presents the first- and second-generation evaluation literature. The chapter begins with a review of the first-generation evaluations, which have been separated into three distinct categories of substance abuse treatment programs: (1) institutionally-based, (2) institutionally-based with a community aftercare component, and (3) community-based programming. The aim of this review is to not only illustrate the considerable amount of attention that has been given to summative evaluation (i.e., the examination of the ultimate program goals of recidivism and relapse), but also to highlight the value of substance abuse programs as a tool for influencing recidivism and relapse.

Followed by this review is a discussion of the second-generation evaluation literature. This body of work is smaller, with the majority of evaluations conducted on institutionally-based substance abuse programs. Nonetheless, the presentation of this literature serves to demonstrate the range of additional program outcomes that have been examined as well as the potential capacity of these programs to affect other program goals.

\section{First-Generation Evaluations}

Over the last decade and a half, there has been a steady production of firstgeneration evaluations. Although a variety of treatment programs have been evaluated, such as counselling, boot camps, education programs, and 12-step programs, the TC has been a primary focus. The recurring finding from this body of literature is that the TC 
effectively reduces recidivism rates as well as relapse rates. All of the evaluations presented in the next three subsections of this chapter use the TC as its subject.

Evaluations of institutionally-based TCs. A recent meta-analysis efficiently summarizes this literature. Pearson and Lipton (1999) coded primary evaluations on three types of institutionally-based substance abuse programs, including the TC, reported between 1968 and 1996. After applying specific selection criteria, 20 studies with a sample size of 2,955 were included in the meta-analysis. Each of the studies was rated on the overall quality of the research methods used. The continuum for this variable was: 1 (poor), 2 (fair), 3 (good), and 4 (excellent). The original hypothesis was that each specific type of program (i.e., TC, counselling, and boot camps) would have a successful impact on recidivism. Recidivism was operationalized as rearrest and/or reincarceration.

Results supported the efficacy of the TC, but not boot camps or counselling. Indeed, TC substance abuse treatment produced an overall mean effect size of $r=.13$ for reduced recidivism. Using the $\mathrm{BED}$, the mean effect size translated into a recidivism rate of $56.5 \%$ in the comparison group compared to $43.5 \%$ in the treatment group. Studies rated as having poor quality had a mean effect size of $r=.07$. Studies with either fair or good ratings had larger effects in comparison to the poor quality studies, with a mean effect size of $r=.19$ and $r=.16$, respectively.

Evaluations of institutionally-based TCs with community aftercare. The Amity prison-based TC and its community aftercare TC component known as Vista have been regularly evaluated since inception. In a more recent evaluation of Amity and Vista, offenders were randomly assigned to either a treatment group or a no-treatment control group. Data were collected on offenders prior to admission, during Amity participation in 
prison, during Vista participation in the community, and at both follow-up periods. In addition, client characteristics identified as predictors of recidivism, such as age, criminal history, antisocial personality, and criminogenic needs were controlled for (Wexler et al., 1999).

Results from the evaluation revealed a lower recidivism rate for the treatment group than the control group at the first- and second-year follow-up periods (see Table 2). Interestingly, those offenders who completed Amity and Vista had an even lower rate of reincarceration at 12 months $(8.2 \%)$ and at 24 months $(14.0 \%)$ than offenders who just completed Amity. The authors note that the 12-month recidivism rate could be confounded by time at risk, as the offenders in the treatment group were still voluntarily participating in the aftercare component of the program.

In an extension of this evaluative work, Wexler, Melnick, Lowe, and Peters (1999) examined the stability of the previous treatment outcomes at a 36-month followup period, and compared these additional analyses with the earlier findings. Comparisons across the 12-, 24-, and 36-month follow-up periods demonstrated consistent positive outcomes associated with the completion of the TC program (see Table 2). As in the previous evaluation, analyses revealed lower recidivism rates for offenders who completed both the in-prison (i.e., Amity) and community (i.e., Vista) TC programs $(27 \%)$. This rate was lower than offenders who only completed Amity and offenders who dropped out of the prison treatment program ( $79 \%$ and $82 \%$, respectively). The results of these two evaluations demonstrate the ability of TC programs to reduce recidivism, and they highlight the importance of continuity of care.

The In-Prison Therapeutic Community (IPTC) is located in Kyle, Texas and 
Table 2

Effectiveness of Institutionally-Based TC with Community Aftercare in Reducing Recidivism and Relapse

\begin{tabular}{|c|c|c|c|c|c|c|}
\hline $\begin{array}{l}\text { Program and } \\
\text { author (s) }\end{array}$ & $\begin{array}{l}\text { Research } \\
\text { design }\end{array}$ & $\vec{N}$ & $\begin{array}{l}\text { Program } \\
\text { description }\end{array}$ & Recidivism & Relapse & $\begin{array}{l}\text { Fw- } \\
\text { up }\end{array}$ \\
\hline \multirow[t]{3}{*}{$\begin{array}{l}\text { Amity-Vista } \\
\text { - } \quad \text { Wexler et } \\
\text { al. (1999) }\end{array}$} & $\begin{array}{l}\text { PRO exp } \\
\text { dsgn; } \\
\text { longitud. }\end{array}$ & 715 & $\begin{array}{l}\text { Amity }=\text { prison } \\
\text { TC consisting of } \\
8-12 \text { months of } \\
\text { tx }\end{array}$ & $\begin{array}{l}\text { Reincarceration } \\
\text { (return to prison for } \\
\text { parole violation or } \\
\text { rearrest) }\end{array}$ & Not examined & $1 \mathrm{yr}$ \\
\hline & & & $\begin{array}{l}\text { Vista }= \\
\text { community TC } \\
\text { consisting of up } \\
\text { to } 12 \text { months of } \\
\text { tx }\end{array}$ & $\begin{array}{l}\text { - } \quad \text { Tx: } 33.9 \% \%^{\mathrm{a}} \\
\text { - }\end{array}$ & & \\
\hline & & 263 & & $\begin{array}{l}\text { Reincarceration } \\
\text { (return to prison for } \\
\text { parole violation or } \\
\text { rearrest) } \\
\text { - } \mathrm{Tx} \text { (any): } 43.3 \%^{\mathrm{a}} \\
\text { - No Tx: } 67.1 \%^{\mathrm{b}}\end{array}$ & Not examined & $2 \mathrm{yrs}$ \\
\hline $\begin{array}{l}\text { Amity-Vista } \\
\text { - Wexler, } \\
\text { Melnick et } \\
\text { al. (1999) }\end{array}$ & $\begin{array}{l}\text { PRO exp } \\
\text { dsgn; } \\
\text { longitud. }\end{array}$ & 478 & & $\begin{array}{l}\text { Reincarceration } \\
\text { (return to prison for } \\
\text { parole violation or } \\
\text { rearrest) } \\
\text { - Tx: } 69.0 \% \text { af } \\
\text { - No Tx: } 75.0 \% \text { bf }\end{array}$ & Not examined & 3 yrs \\
\hline $\begin{array}{l}\text { IPTC } \\
\text { Eisenberg } \\
\& \text { Fabelo } \\
(1996)\end{array}$ & $\begin{array}{l}\text { PRO } \\
\text { quasi exp } \\
\text { dsgn; } \\
\text { longitud. }\end{array}$ & 672 & $\begin{array}{l}9-12 \text { month in- } \\
\text { prison TC } \\
\text { followed by } 4 \\
\text { months of } \\
\text { postrelease tx }\end{array}$ & $\begin{array}{l}\text { Reincarceration } \\
\text { (return to prison) } \\
\text { Tx: } 7.0 \%^{\mathrm{c}} \\
\text { - Partial Tx: } 19 \% \\
\text { No Tx: } 19.0 \%^{\mathrm{d}}\end{array}$ & Not examined & $1 \mathrm{yr}$ \\
\hline $\begin{array}{l}\text { PPC } \\
\text { Eisenberg } \\
(1999)\end{array}$ & $\begin{array}{l}\text { PRO } \\
\text { quasi exp } \\
\text { dsgn; } \\
\text { longitud. }\end{array}$ & 482 & & $\begin{array}{l}\text { Reincarceration } \\
\text { (return to prison) } \\
=\quad \mathrm{Tx}: 10.0 \%{ }^{\mathrm{c}} \\
\text { - No Tx: } 19.0 \%{ }^{\mathrm{e}}\end{array}$ & Not examined & $1 \mathrm{yr}$ \\
\hline
\end{tabular}


Evaluation of a Correctional Substance Abuse Program 63

\begin{tabular}{|c|c|c|c|c|c|c|}
\hline $\begin{array}{l}\text { Program and } \\
\text { author (s) }\end{array}$ & $\begin{array}{l}\text { Research } \\
\text { design }\end{array}$ & $N$ & $\begin{array}{l}\text { Program } \\
\text { description }\end{array}$ & Recidivism & Relapse & $\begin{array}{l}\text { Fw- } \\
\text { up }\end{array}$ \\
\hline & & 482 & & $\begin{array}{l}\begin{array}{l}\text { Reincarceration } \\
\text { (return to prison) }\end{array} \\
\text { Tx: } 18.0 \%^{\mathrm{c}} \\
\text { No Tx: } 26.0 \%^{\mathrm{e}}\end{array}$ & Not examined & 2 yrs \\
\hline & & 482 & & $\begin{array}{l}\begin{array}{l}\text { Reincarceration } \\
\text { (return to prison) }\end{array} \\
\quad \mathrm{Tx}: 33.0 \%^{\mathrm{cf}} \\
\text { No Tx: } 37.0 \%{ }^{\mathrm{ef}}\end{array}$ & Not examined & 3 yrs \\
\hline $\begin{array}{l}\text { IMPACT } \\
\begin{array}{r}\text { Swartz et } \\
\text { al. (1996) }\end{array}\end{array}$ & $\begin{array}{l}\text { RETRO } \\
\text { quasi exp } \\
\text { within- } \\
\text { subjects } \\
\text { dsgn }\end{array}$ & 438 & $\begin{array}{l}\text { Based on TC } \\
\text { model; } \\
\text { residential drug } \\
\text { tx with } 30 \text {-day } \\
\text { orientation, } 90 \\
\text { days of intensive } \\
\text { individual and } \\
\text { group } \\
\text { counselling, and } \\
\text { aftercare } \\
\text { focusing on } \\
\text { reintegration } \\
\text { and relapse }\end{array}$ & $\begin{array}{l}\text { Rearrests } \\
\begin{array}{l}\text { - } \quad 1-30 \text { tx days: } \\
69.0 \%^{\mathrm{g}} \\
\text { - } \quad 31-90 \text { tx days: } \\
55.0 \% \%^{\mathrm{g}} \\
\quad \begin{array}{l}91-150 \text { or more } \mathrm{tx} \\
\text { days: } 41.0 \% \mathrm{0}^{\mathrm{g}}\end{array}\end{array}\end{array}$ & Not examined & $\begin{array}{l}\text { Avg } \\
\text { of } \\
535 \\
\text { dys }\end{array}$ \\
\hline $\begin{array}{l}\text { DAP } \\
\text { - Pelissier et } \\
\text { al. }(2000)\end{array}$ & $\begin{array}{l}\text { PRO } \\
\text { quasi exp } \\
\text { dsgn; } \\
\text { longitud. }\end{array}$ & 1842 & $\begin{array}{l}\text { Uses TC model } \\
\text { and incorporates } \\
\text { cognitive- } \\
\text { behavioural } \\
\text { components; } 3 \\
\text { phases = (1) 9- } \\
12 \text { month unit- } \\
\text { based } \\
\text { component, (2) } \\
\text { up to } 12 \text { months } \\
\text { of tx in general } \\
\text { population, (3) } \\
\text { community tx } \\
\text { when transferred } \\
\text { from prison }\end{array}$ & $\begin{array}{l}\text { New offense } \\
\text { Tx: } 30.6 \%^{\mathrm{c}} \\
\text { No Tx: } 37.6 \%^{\mathrm{i}}\end{array}$ & $\begin{array}{l}\text { First use after } \mathrm{tx}^{\mathrm{h}} \\
\text { - Tx: } 49.9 \%^{\mathrm{c}} \\
\text { - No Tx: } 58.5 \%^{\mathrm{i}}\end{array}$ & $3 \mathrm{yrs}$ \\
\hline $\begin{array}{l}\text { KEY-CREST } \\
\begin{array}{l}\text { Inciardi et } \\
\text { al. (1997) }\end{array}\end{array}$ & $\begin{array}{l}\text { PRO } \\
\text { quasi exp } \\
\text { dsgn; } \\
\text { longitud. }\end{array}$ & 448 & $\begin{array}{l}\text { KEY = prison- } \\
\text { based TC } \\
\text { CREST = } \\
\text { community- } \\
\text { based work } \\
\text { release TC }\end{array}$ & $\begin{array}{l}\text { SR arrests } \\
\text { KEY: } 57.0 \% \%^{\mathrm{c}} \\
\text { - } \\
\text { CREST: } 43.0 \%{ }^{\mathrm{c}} \\
\text { KEY-CREST: } \\
23.0 \%{ }^{\mathrm{c}} \\
\text { No Tx: } 54.0 \%{ }^{\mathrm{k}}\end{array}$ & $\begin{array}{l}\text { SR drug use and UR } \\
\text { KEY: } 47.0 \%{ }^{\mathrm{c}} \\
\text { CREST: } 49.0 \%^{\mathrm{c}} \\
\text { KEY-CREST: } \\
\text { Ke.0\% } \\
\text { - No Tx: } 65.0 \%{ }^{\mathrm{k}}\end{array}$ & $\begin{array}{l}18 \\
\mathrm{~ms}\end{array}$ \\
\hline
\end{tabular}


Evaluation of a Correctional Substance Abuse Program 64

\begin{tabular}{|c|c|c|c|c|c|c|}
\hline $\begin{array}{l}\text { Program and } \\
\text { author }(\mathrm{s})\end{array}$ & $\begin{array}{l}\text { Research } \\
\text { design }\end{array}$ & $N$ & $\begin{array}{l}\text { Program } \\
\text { description }\end{array}$ & Recidivism & Relapse & $\begin{array}{l}\text { Fw- } \\
\text { up }\end{array}$ \\
\hline $\begin{array}{l}\text { KEY-CREST } \\
\text { - Inciardi et } \\
\text { al. (1999) }\end{array}$ & $\begin{array}{l}\text { PRO } \\
\text { quasi exp } \\
\text { dsgn; } \\
\text { longitud. }\end{array}$ & 428 & & 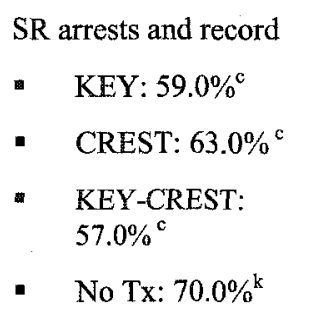 & 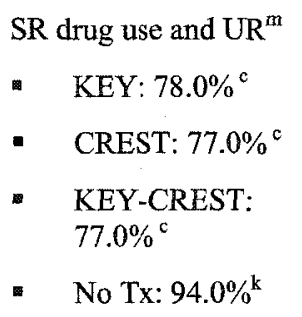 & $3 \mathrm{yrs}$ \\
\hline & & 428 & & $\begin{array}{l}\text { SR arrests and record } \\
\text { - CREST dropout: } \\
72.0 \% \\
\text { - CREST: } 45.0 \%^{\circ} \\
\text { - CREST with } \\
\text { aftercare: } 31.0 \%^{\circ} \\
\text { - No Tx: } 71.0 \%^{\mathrm{k}}\end{array}$ & $\begin{array}{l}\text { SR drug use and UR } \\
\text { - CREST dropout: } \\
83.0 \% \\
\text { - } \quad \text { CREST: } 73.0 \%^{\mathrm{c}} \\
\text { - } \quad \text { CREST with } \\
\text { aftercare: } 65.0 \%^{\mathrm{c}} \\
\text { - } \\
\text { No Tx: } 95.0 \% \%^{\mathrm{k}}\end{array}$ & $3 \mathrm{yrs}$ \\
\hline
\end{tabular}

Note Tx $=$ treatment; PRO $=$ prospective; RETRO = retrospective; Fw-up $=$ follow-up $;$ Exp $=$ experimental; Dsgn $=$ Design; Longitud = longitudinal; Yrs = years; Ms = months; Dys = days; $S R=$ self-report; UR = urinalysis.

${ }^{a}$ The treatment group consisted of offenders who at least completed the Amity program.

${ }^{b}$ The no treatment group consisted of offenders on a waiting list for the Amity-Vista program.

${ }^{\mathfrak{c}}$ The treatment group consisted of offenders who completed the program.

${ }^{d}$ The no treatment group consisted of offenders who met the selection criteria for the IPTC program, but were released prior to program selection.

${ }^{\mathrm{e}}$ The no treatment group consisted of offenders eligible to participate in the IPTC program, but did not.

${ }^{\mathrm{f}}$ Although the treatment group had a lower recidivism rate than the control group, the finding was not statistically significant.

${ }^{g}$ Given that offenders were followed-up for varying lengths of time, which could influence the recidivism rates, the researchers conducted a second analysis with only those offenders who were at risk in the community for at least one year $(n=344)$. The same pattern of rearrest rates across the groups was evident.

${ }^{\mathrm{h}}$ These relapse rates are based only on participation in the institutional component of the DAP program.

${ }^{i}$ The no treatment group consisted of offenders eligible to participate in DAP, but did not.

${ }^{j}$ Relapse rates are based on a 30-day self-report and a 18 -month urinalysis test.

${ }^{k}$ The no treatment group consisted of offenders on conventional work release who did not participate in KEY or CREST. 
modelled after the Stay'N Out TC program in New York. As can be seen in Table 2, analyses revealed that the program completers (i.e., offenders who completed the prison and community aftercare) had significantly lower recidivism rates than the group who did not complete the program and the comparison group (Eisenberg \& Fabelo, 1996). In another evaluation of IPTC, program completers and a comparison group were compared on recidivism rates (Fabelo, 1999). After one full year in the community, program completers (i.e., those who completed the 9- to 12-month prison treatment and the 4month community treatment) had significantly lower recidivism rates than offenders who did not participate in the program (see Table 2). Similar patterns of recidivism were evident for the second and third years of follow-up. However, results at the 36-month mark were not statistically significant. Indeed, the results from the two evaluations of IPTC are consistent with the Amity and Vista evaluations, that is, offenders who participated in both the institutional TC and community aftercare had lower recidivism rates.

The Integrated Multiphase Program of Assessment and Comprehensive Treatment (IMPACT) program is delivered in Chicago's Cook County jail (Swartz et al., 1996). In an evaluation of this program, comparison groups were created from the total sample based on their length of stay in the program (see Table 2). Rates of recidivism significantly decreased with increasing lengths of stay in IMPACT (see Table 2).

Survival analysis was completed to compare the most effective or optimal treatment condition with the least effective or minimal treatment condition. Results revealed that the most effective condition was 90 to 150 days ( 3 to 5 months) in IMPACT followed by immediate community drug treatment. The least effective treatment 
condition was less than 30 days in IMPACT followed by no community treatment. Specifically, offenders who did 3 to 5 months of treatment had a median survival time of 723 days in comparison to 218 days for the group who had 1 to 30 days of treatment (Swartz et al., 1996). Both the recidivism rates and survival analysis illustrate not only the ability of this program to reduce future criminal activity, but also its ability to postpone future involvement in criminal behaviour. In addition, this study demonstrates the importance of length of treatment and continuity of care for the reduction of recidivism.

Recently, the Federal Bureau of Prisons in the United States completed a threeyear follow-up evaluation on 20 of its national residential drug treatment programs (DAP), representing $40 \%$ of the institutions currently delivering DAP (Pelissier et al., 1998, 2000). Those offenders who received treatment had a significantly lower rate of arrests than the control group (see Table 2). Analyses on substance use revealed that offenders who completed DAP were less likely to use drugs or alcohol within 36 months of release than untreated offenders (see Table 2). These results support the efficacy of substance abuse treatment with respect to reduced recidivism and relapse rates among offenders.

The KEY-CREST TC operates in Delaware within the Delaware Correctional system (Lipton, 1998). KEY-CREST consists of a prison-based TC (KEY) and a community-based work release TC (CREST). Several evaluations of KEY-CREST have been completed to date (Inciardi et al., 1997; Lockwood et al., 1997; Martin, Butzin, \& Inciardi, 1995; Martin, Butzin, Saum, \& Inciardi, 1999; Nielsen et al., 1996). In the analyses of one evaluation, Inciardi and colleagues (1997) controlled for group 
differences with several variables including: gender (although the sample was primarily male), age, ethnicity, criminal history, and previous drug use. After taking into account group differences, the results revealed that the CREST participants and the KEY-CREST participants reported fewer arrests than the KEY group and the comparison group (see Table 2). Examination of relapse rates showed that the KEY-CREST group relapsed at a much lower rate than the other three groups (see Table 2). These results are not overly supportive of the effectiveness of the institutional TC program (KEY) in reducing recidivism, given that the KEY group was not statistically distinguishable from the control group on this outcome measure. However, the evaluation suggests that those offenders who receive the full continuum of treatment (KEY-CREST) have the strongest and most consistent patterns of success.

In another evaluation of the KEY-CREST program, researchers addressed the need for a longer-term follow-up period, and decided to do a more thorough inspection of the community component of the program (Martin et al., 1999). Similar to the previous evaluation, group differences were controlled for with the following variables: gender (although the sample was primarily male), ethnicity, and previous drug use. Results from this evaluation are reported in Table 2. From these results, it appears that the effect of the overall program declines when the time at risk is moved to three years after release. Although the KEY, CREST, and KEY-CREST groups reported lower rates of arrests, the differences were not significantly different from the comparison group. On the other hand, significantly different relapse rates were found between these three groups and the comparison group, with fewer of the KEY, CREST, and KEY-CREST participants returning to use. 
Additional analyses revealed that offenders who participated in community treatment (i.e., CREST) reported lower rates of recidivism and relapse than those with no treatment or CREST dropouts (see Table 2). In addition, offenders who completed CREST with aftercare have even lower rates of recidivism and relapse than the three other groups (see Table 2). Overall, this evaluation continues to support the efficacy of substance abuse programming and the importance of continuity of care.

Recently, predictive analyses have been completed on the KEY and CREST programs (Inciardi, Martin, \& Butzin, 2004). In one analysis, 590 offenders who participated in either of these two programs were tracked on arrest and relapse status at a 5-year follow-up. Arrest was measured using self-report and arrest records. Drug relapse was measured using self-report and urinalysis tests. The researchers reported that for arrest status, having participated in treatment and being older were significant predictors of not being arrested five years after program participation. For relapse status, significant predictors of not using drugs five years after program participation were participation in treatment, lower levels of prior drug use, and participation in the aftercare program (i.e., CREST).

Evaluations of community-based TCs. The Dallas County Judicial Treatment Center has a Substance Abuse Treatment Facility (SATF) for probationers who have been identified with substance abuse problems. A posttreatment evaluation was completed to examine the effectiveness of this community program (Hiller et al., 1996). Results indicated that significantly fewer program graduates were rearrested at the 6-month follow-up than participants who were either expelled or transferred (see Table 3). An examination of participants who completed both the community program (i.e., SATF) 
Evaluation of a Correctional Substance Abuse Program 69

Table 3

\section{Effectiveness of Community-Based TC in Reducing Recidivism and Relapse}

\begin{tabular}{|c|c|c|c|c|c|c|}
\hline $\begin{array}{l}\text { Program and } \\
\text { author (s) }\end{array}$ & $\begin{array}{l}\text { Research } \\
\text { design }\end{array}$ & $N$ & $\begin{array}{l}\text { Program } \\
\text { description }\end{array}$ & Recidivism & Relapse & $\begin{array}{l}\text { Fw- } \\
\text { up }\end{array}$ \\
\hline $\begin{array}{l}\text { SATF } \\
\begin{array}{l}\text { Hiller et } \\
\text { al. } \\
(1996)\end{array}\end{array}$ & $\begin{array}{l}\text { PRO } \\
\text { quasi } \\
\text { exp } \\
\text { dsgn; } \\
\text { longitud. }\end{array}$ & 492 & $\begin{array}{l}\text { Community } \\
\text { program based on } \\
\text { TC model with an } \\
\text { aftercare } \\
\text { component } \\
\text { referred to as } \\
\text { Step Down }\end{array}$ & $\begin{array}{l}\text { Rearrests } \\
\text { - Tx: } 6.0 \%^{\mathrm{b}} \\
\text { - Expelled: } 13.0 \% \\
\text { - Transferred: } 27.0 \%\end{array}$ & Not examined & $6 \mathrm{~ms}$ \\
\hline & & 351 & & Not examined & $\begin{array}{l}\text { Drug use after } \mathrm{tx}^{\mathrm{a}} \\
\text { - } \quad \text { SAFT: } 41.0 \% \\
\text { SAFT and } \\
\text { StepDown: } 26.0 \% \\
\text { Alcohol use after tx } \\
\text { - SAFT: } 26.0 \% \\
\text { - } \\
\text { SAFT and } \\
\text { StepDown: } 19.0 \%\end{array}$ & $6 \mathrm{~ms}$ \\
\hline $\begin{array}{l}\text { SATF } \\
\begin{array}{l}\text { Knight } \\
\text { \& Hiller } \\
(1997)\end{array}\end{array}$ & $\begin{array}{l}\text { PRO } \\
\text { quasi } \\
\text { exp } \\
\text { dsgn; } \\
\text { longitud. }\end{array}$ & 492 & & $\begin{array}{l}\text { Rearrests } \\
\text { - } \quad \text { Tx: } 11.0 \%^{\mathrm{b}} \\
\text { - } \quad \text { Expelled: } 21.0 \% \\
\text { - } \quad \text { Transferred: } 37.0 \%\end{array}$ & Not examined & $1 \mathrm{yr}$ \\
\hline $\begin{array}{l}\text { CREST } \\
\text { - Nielsen } \\
\text { et al. } \\
(1996)\end{array}$ & $\begin{array}{l}\text { PRO } \\
\text { quasi } \\
\text { exp } \\
\text { dsgn; } \\
\text { longitud. }\end{array}$ & 689 & $\begin{array}{l}\text { Community- } \\
\text { based work } \\
\text { release TC }\end{array}$ & $\begin{array}{l}\text { SR rearrests and } \\
\text { subsequent charges } \\
\text { - CREST: } 14.7 \% \\
\text { - No Tx: } 35.4 \%{ }^{\circ} \\
\text { SR rearrests and } \\
\text { subsequent charges } \\
\text { - CREST: } 38.2 \% \\
\text { - No Tx: } 63.0 \%{ }^{\mathrm{c}}\end{array}$ & $\begin{array}{l}\text { SR substance use } \\
\text { after tx } \\
\text { - CREST: } 16.2 \% \\
\text { No Tx: } 62.2 \%{ }^{\mathrm{c}} \\
\text { SR substance use } \\
\text { after tx } \\
\text { - CREST: } 51.7 \% \\
\text { No Tx: } 79.0 \%\end{array}$ & $\begin{array}{l}18 \\
\mathrm{~ms}\end{array}$ \\
\hline
\end{tabular}

Note. $\mathrm{Tx}=$ treatment; $\mathrm{PRO}=$ prospective $; \mathrm{Fw}$-up $=$ follow-up; $\mathrm{Exp}=$ experimental; Dsgn $=$ design; Longitud $=$ longitudinal; $\mathrm{Yrs}=$ years; $\mathrm{Ms}=$ months; $\mathrm{SR}=$ self-report.

${ }^{a}$ Data on substance use after participating in treatment was collected from sources including: personal interviews, probation officer contacts, and counsellor records.

${ }^{b}$ The treatment group consisted of offenders who completed the program.

${ }^{\mathrm{c}}$ The no treatment group consisted of offenders on conventional work release who did not participate in CREST. 
and aftercare (i.e., Step Down) revealed a significantly lower rate of relapse to drug and alcohol use compared to offenders who only completed SATF (see Table 3). These findings suggest that community-based TC programming can influence reductions in recidivism and relapse among substance-abusing offenders. Interestingly, the authors noted that their study did not provide insight into the effects of community-based TC programming on what offenders actually learn related to substance abuse (Hiller et al., 1996). This statement highlights a disadvantage of doing purely summative evaluation.

Knight and Hiller (1997) addressed the short follow-up of the 1996 SATF evaluation by conducting a 1-year outcome evaluation on the same program and sample. Analysis revealed that one year after completing SATF, graduates still had significantly fewer arrests than offenders who were either expelled or transferred (see Table 3). In this evaluation, the researchers also conducted a logistic regression analysis to determine what background variables predicted rearrests and program completion. Analyses indicated that participation in the aftercare component was a significant predictor of fewer rearrests. Specifically, program completers who were not admitted to aftercare had 2.1 higher odds of being rearrested a year after treatment completion than offenders who did aftercare. In addition, results from the logistic regression model revealed that gender (although the majority of the sample was male), age, and education level were significant predictors of program completion (Knight \& Hiller, 1997). Thus, it appeared that being male, older, and having more education predicted program completion.

As previously described, CREST is a community-based work release program modelled on the TC. CREST is often used as the aftercare component of KEY, a prisonbased TC (Nielsen et al., 1996). At the 6-month follow-up, the results illustrated 
significant differences for both the rearrest and relapse rates between CREST participants and the control group, with CREST participants reporting lower rearrest and relapse rates than the control group (see Table 3). Although the percentage of reported rearrests and relapses were higher for both groups at the 18-month follow-up, significant differences between the treatment and control group remained (see Table 3 ). This evaluation illustrates the potential of a stand-alone community-based TC program to reduce recidivism and relapse rates among substance-abusing offenders.

The District of Columbia Treatment Initiative has conducted several predictive studies examining the efficacy of providing community-based TC treatment (Messina, Wish, \& Nemes, 1999, 2001; Nemes, Messina, Wish, \& Wraight, 1999). In one study, researchers identified variables that predicted posttreatment arrests and relapse at a 19month follow-up (Messina et al., 1999). Criminal records obtained from the D.C. Pretrial Services Agency were used to code arrests. Urinalysis tests done at follow-up served as the relapse measure. With a sample of 242 male offenders, the regression analyses revealed that treatment completion and age were associated with reductions in rearrests. That is, offenders who completed treatment had a 3.70 higher odds of not being rearrested than offenders who did not complete treatment. In the same study using a sample of 172 male offenders, the only variable significantly predicting relapse was treatment completion. Specifically, offenders who graduated from treatment had a 5.88 higher odds of not having a positive urinalysis at follow-up than offenders who did not graduate from treatment.

From this review of the first-generation TC evaluation literature, it can be reasonably concluded that institutional and community-based TC programming is 
effective in reducing future criminal behaviour and substance use. Furthermore, this evidence is supportive of the criminal justice system's investment in substance abuse programming. However, other program performance variables, such as additional summative outcomes and formative issues remain unexplored. Many researchers contend that more efficient evaluation of substance abuse treatment programs is required to broaden understanding of how substance abuse programming works, what offenders actually learn related to substance abuse as well as to inform continued programming development and implementation (Etheridge \& Hubbard, 2000; Farabee et al., 1999; Gaes, 1998; Gendreau et al., 1999; Harris \& Smith, 1996; Hiller et al., 1996; Sia, Dansereau, \& Czuchry, 2000). Examination of these outstanding issues has commenced with second-generation evaluation. Second-generation evaluations are reviewed in the next section of this chapter.

\section{Second-Generation Evaluations}

The second-generation evaluation literature is a smaller body of research than the first-generation. Nonetheless, the existing second-generation evaluation literature has two predominant features. First, the TC is not the only treatment model studied. Evaluations on the effectiveness of cognitive-behavioural substance abuse treatment programs emerge in this generation. Second, although most of these evaluations have measured the impact of substance abuse programming on recidivism and/or relapse, additional program performance variables and formative issues, such as knowledge, confidence, client perceptions/satisfaction, and motivation are also measured. Given that the first-generation evaluation literature illustrates that substance abuse programming can potentially reduce recidivism and/or relapse rates, review of the second-generation evaluation literature will 
concentrate on describing the methods and results of the additional program performance variables and formative issues included in these evaluations.

The Offender Substance Abuse Pre-Release Program (OSAPP) is delivered nationally within the Canadian federal correctional system. Since OSAPP's inception in the early 1990 s, it has been evaluated on several occasions. The first evaluation of OSAPP took place during its pilot phase at Drumheller Institution in Alberta (Millson \& Robinson, 1992). Offenders were randomly assigned to participate in the pilot of OSAPP or not. Both the treatment group and control group completed a battery of assessments before and after the program.

This battery of measures was adapted from Gunn, Orenstein, Iverson, and Mullen (as cited in $\mathrm{T}^{3}$ Associates Inc., 1999b). The eight assessment instruments were: (1) the Consequences of Alcohol Use Scale (assesses knowledge on the consequences of alcohol use), (2) the Consequences of Drug Use Scale (measures knowledge on the consequences of drugs), (3) the How Much Do They Matter Questionnaire (examines knowledge on how people might be affected by using alcohol or drugs), (4) the Drinking and Assertiveness Scale (assesses knowledge of how to decline offers made by others to use alcohol in an assertive manner and a socially appropriate manner), (5) the Communicating About Drinking Questionnaire (measures knowledge of how to communicate to others their wish to stop or control their use), (6) the Using Alcohol Responsibly measure (examines knowledge of using alcohol responsibly), (7) the Decision Making Scale (assesses knowledge of decision making related to personal health), and (8) the Employment Questionnaire (measures knowledge of relapse situations). Results showed that the offenders who participated in OSAPP attained higher 
scores on several of the measures after program participation than offenders who did not participate in the program (see Table 4). These results suggest that changes in knowledge were attributable to program participation.

A larger sample of 317 offenders who participated in OSAPP at Bath Institution in Ontario between 1990 and 1992 were included in a second evaluation of the program (Millson et al., 1995). As in the pilot study, all eight assessment instruments were completed by offenders before and after program participation. Results demonstrated that after completing the program, offenders significantly increased their knowledge in all of the examined areas with the exception of decision making related to personal health (see Table 4). Specifically, these findings suggest that the program was successful in increasing offender knowledge about the effects and consequences of alcohol and drug use as well as their knowledge of essential skills for abstaining or controlling future alcohol and/or drug use.

In addition, pre- and posttests were analyzed in relation to readmission rates to determine whether individual performance on the assessment instruments was associated with postrelease outcome. The average follow-up period after release was 15 months. Survival analysis revealed noticeably higher survival rates for offenders who performed well in the program. After 8 months on release, offenders who had the poorest program performance also had the poorest survival rate, that is, they had the highest readmission rate compared to the other groups (Millson et al., 1995).

A more recent evaluation of OSAPP has been completed (T3 Associates Inc., 1999b). One of the interests of this evaluation was to explore changes in knowledge as a result of program participation. Pre- and posttesting completed by 2,432 OSAPP 
Evaluation of a Correctional Substance Abuse Program 75

Table 4

Summary of Findings from Second-Generation Evaluations

\begin{tabular}{|c|c|c|c|c|c|}
\hline $\begin{array}{l}\text { Program and } \\
\text { author }(\mathrm{s})\end{array}$ & $\begin{array}{l}\text { Research } \\
\text { design }\end{array}$ & $N$ & $\begin{array}{l}\text { Program } \\
\text { description }\end{array}$ & $\overline{D V}$ & Findings \\
\hline $\begin{array}{l}\text { OSAPP } \\
\begin{array}{l}\text { Millson \& } \\
\text { Robinson } \\
(1992)\end{array}\end{array}$ & $\begin{array}{l}\text { PRO exp } \\
\text { dsgn; pre } \\
\text { and } \\
\text { posttest }\end{array}$ & 40 & $\begin{array}{l}\text { Institutionally } \\
\text {-based pre- } \\
\text { release } \\
\text { cognitive- } \\
\text { behavioural } \\
\text { program }\end{array}$ & $\begin{array}{l}\text { Knowledge of: } \\
\text { 1. Consequences of } \\
\text { alcohol and drug } \\
\text { use } \\
\text { 2. Effects of } \\
\text { substance use } \\
\text { 3. Assertiveness } \\
\text { 4. Communication } \\
\text { 5. Responsible use } \\
\text { 6. Decision making } \\
\text { 7. Relapse situations }\end{array}$ & $\begin{array}{l}\text { Tx group had significantly } \\
\text { higher scores on assessments } \\
\text { looking at knowledge of alcohol } \\
\text { and drug effects and relapse } \\
\text { situations than the control group. } \\
\text { Positive differences were found } \\
\text { for the tx group with knowledge } \\
\text { of assertiveness, using alcohol } \\
\text { responsibly, and decision } \\
\text { making related to health; } \\
\text { although, group differences were } \\
\text { not significant. }\end{array}$ \\
\hline $\begin{array}{l}\text { OSAPP } \\
\begin{array}{l}\text { Millson et } \\
\text { al. (1995) }\end{array}\end{array}$ & $\begin{array}{l}\text { RETRO } \\
\text { quasi } \\
\text { exp } \\
\text { within } \\
\text { subjects } \\
\text { dsgn; pre } \\
\text { and } \\
\text { posttest }\end{array}$ & 317 & & Same as above & $\begin{array}{l}\text { Offenders had significantly } \\
\text { higher scores on knowledge of } \\
\text { the consequences of alcohol and } \\
\text { drugs, the effects of alcohol and } \\
\text { drugs, assertiveness, } \\
\text { communication, using alcohol } \\
\text { responsibly, and relapse } \\
\text { situations after participation in } \\
\text { OSAPP than before. }\end{array}$ \\
\hline $\begin{array}{l}\text { OSAPP } \\
=\quad T^{3} \\
\text { Associates } \\
(1999 b)\end{array}$ & $\begin{array}{l}\text { RETRO } \\
\text { quasi } \\
\text { exp } \\
\text { within- } \\
\text { subjects } \\
\text { dsgn; pre } \\
\text { and } \\
\text { posttest }\end{array}$ & 2432 & & $\begin{array}{l}\text { Knowledge of: } \\
\text { 1. } \begin{array}{l}\text { Consequences of } \\
\text { alcohol and drug } \\
\text { use }\end{array} \\
\text { 2. } \begin{array}{l}\text { Effects of } \\
\text { substance use }\end{array} \\
\text { 3. Assertiveness } \\
\text { 4. Communication } \\
\text { 5. Responsible use } \\
\text { 6. Relapse situations } \\
\text { 7. Responses to }\end{array}$ & $\begin{array}{l}\text { Offenders had significantly } \\
\text { higher scores on knowledge of } \\
\text { the consequences of alcohol and } \\
\text { drugs, the effects of alcohol and } \\
\text { drugs, assertiveness, } \\
\text { communication, using alcohol } \\
\text { responsibly, relapse situations, } \\
\text { responding to relapse situations, } \\
\text { and problem-solving after } \\
\text { participation in OSAPP than } \\
\text { before. }\end{array}$ \\
\hline
\end{tabular}


Evaluation of a Correctional Substance Abuse Program 76

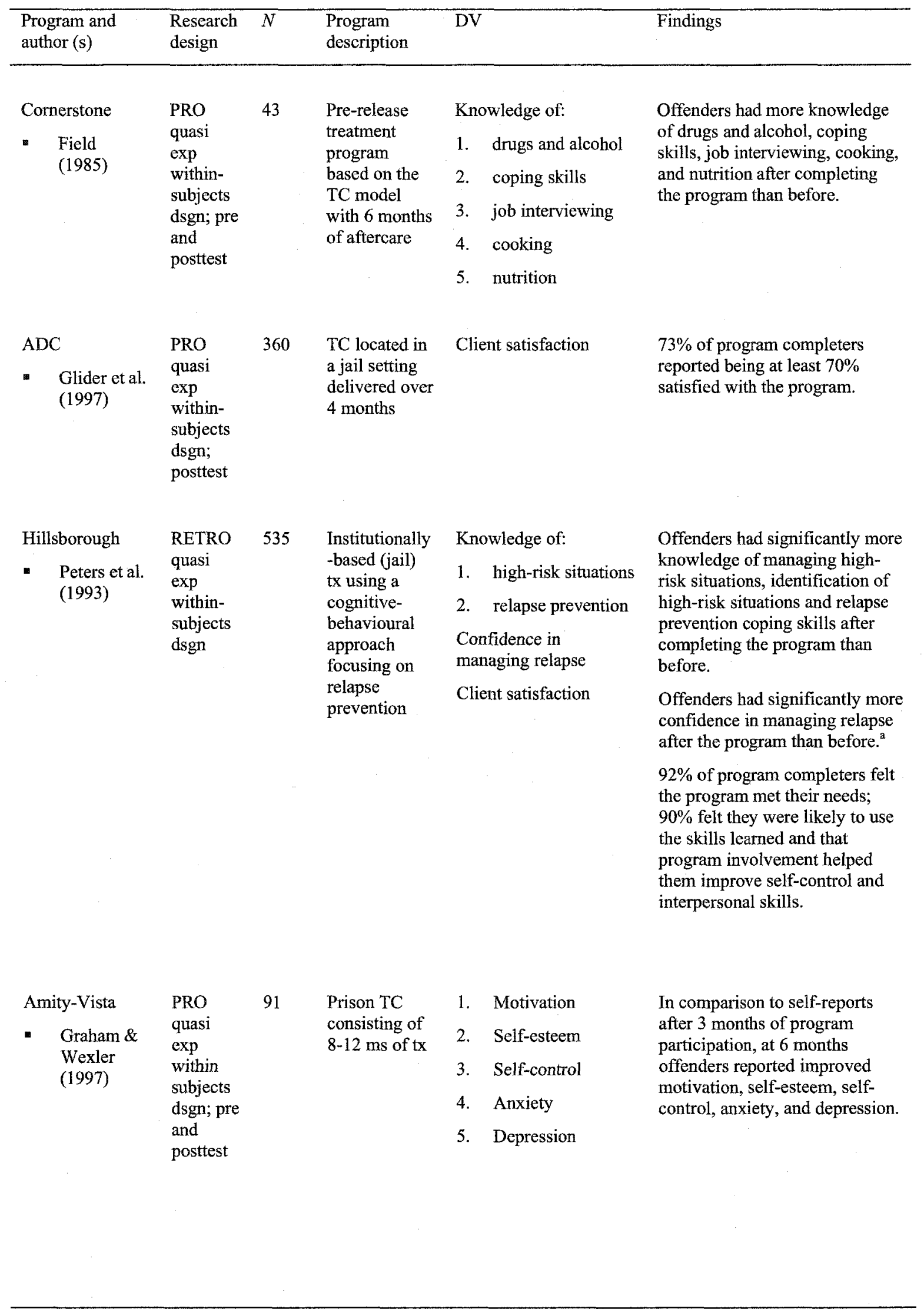


Evaluation of a Correctional Substance Abuse Program 77

\begin{tabular}{|c|c|c|c|c|c|}
\hline $\begin{array}{l}\text { Program and } \\
\text { author (s) }\end{array}$ & $\begin{array}{l}\text { Research } \\
\text { design }\end{array}$ & $N$ & $\begin{array}{l}\text { Program } \\
\text { description }\end{array}$ & DV & Findings \\
\hline \multirow[t]{2}{*}{$\begin{array}{l}\text { CHOICES } \\
\text { - } \quad \begin{array}{l}\mathrm{T}^{3} \\
\text { Associates } \\
(1999 \mathrm{c})\end{array}\end{array}$} & \multirow[t]{2}{*}{$\begin{array}{l}\text { RETRO } \\
\text { quasi } \\
\text { exp } \\
\text { within } \\
\text { subjects } \\
\text { dsgn; pre } \\
\text { and } \\
\text { posttest }\end{array}$} & \multirow[t]{2}{*}{399} & \multirow[t]{2}{*}{$\begin{array}{l}\text { Community- } \\
\text { based } \\
\text { cognitive- } \\
\text { behavioural } \\
\text { program } \\
\text { consisting of } \\
\text { an intensive } \\
10 \text { half day } \\
\text { phase } \\
\text { followed by a } \\
\text { minimum of } 3 \\
\text { ms of weekly } \\
\text { maintenance } \\
\text { sessions }\end{array}$} & $\begin{array}{l}\text { Knowledge of: } \\
\text { 1. Consequences of } \\
\text { alcohol use } \\
\text { 2. Consequences of } \\
\text { drug use } \\
\text { 3. Effects of } \\
\text { substance use } \\
\text { 4. Responsible use } \\
\text { 5. Decision making } \\
\text { 6. Relapse situations } \\
\text { 7. Responses to }\end{array}$ & \multirow[t]{2}{*}{$\begin{array}{l}\text { Offenders had significantly } \\
\text { higher scores on knowledge of } \\
\text { the consequences of alcohol and } \\
\text { drugs, the effects of alcohol and } \\
\text { drugs, using alcohol responsibly, } \\
\text { relapse situations, responding to } \\
\text { relapse situations, and problem- } \\
\text { solving after participation in } \\
\text { CHOICES than before }\end{array}$} \\
\hline & & & & 8. Problem-solving & \\
\hline
\end{tabular}

Note. $\mathrm{Tx}=$ treatment; $\mathrm{PRO}=$ prospective; $\mathrm{Fw}$-up $=$ follow-up; $\mathrm{Yrs}=$ years; Ms $=$ months; $\mathrm{SR}=$ self-report.

${ }^{a}$ Out of the eight categories on the SCQ, offenders who completed the program indicated the greatest improvement in confidence in managing urges and temptations and positive interpersonal situations. 
participants between 1993 and 1997 was utilized in the analyses. Three additional measures were added to the original assessment battery around 1993, which measure knowledge of responses to situations that could lead to relapse (Relapse Attitudes and Knowledge Questionnaire), knowledge of problem-solving (Problem-Solving Scale; D'Zurrilla \& Nezu, 1990), and knowledge of positive and negative effects of substance use (Decisional Balance Scale). At this time, the Decision Making Scale was removed from the battery. Changes in program performance were similar to the results of the 1992 and 1995 evaluations. Examination of the average knowledge scores revealed significant increases in knowledge (see Table 4). These results suggest that the program was able to increase knowledge about the effects and consequences of substance use and knowledge of essential skills for changing substance-abusing behaviour.

Supplementary analyses were performed to determine whether any differences exist between OSAPP participants who completed the pre- and posttesting battery and offenders who finished OSAPP but refused to complete the assessments. Results indicated that these two groups were not similar on several variables. Specifically, in comparison to offenders who completed the pre- and posttesting, offenders who did not complete the battery were somewhat older, disproportionately incarcerated in medium security institutions, had lower levels of education, were lower risk, and were convicted of more violent and less robbery offences. Consequently, it could not be concluded with certainty that the results reported for gained knowledge are representative of all OSAPP participants ( $T^{3}$ Associates Inc., 1999b).

Gary Field (1985) evaluated the Cornerstone Program located in Oregon. Field (1985) examined 43 program graduates from 1983 to 1985 . Knowledge gained from the 
program modules was assessed. The program modules covered the following issues:

education on alcohol and drugs, coping skills, job interview skills, cooking, and nutrition. From the 78-item test designed to measure changes in knowledge, results indicated that participants who completed Cornerstone had more knowledge in all tested areas than before participation (see Table 4). Consequently, the researchers concluded that offenders increased their repertoire of basic life skills information by completing Cornerstone.

The Adult Detention Centre (ADC) of Pima County, Arizona developed a TC substance abuse program (Glider et al., 1997). One of the program goals of the ADC TC was to get inmates to continue with some type of community treatment once released. Client satisfaction was measured in addition to recidivism. Glider and colleagues (1997) felt that participants' perception of satisfaction with the treatment program was an important indicator of treatment effectiveness. Satisfaction with the program was measured with the Moos Correctional Environment Scale (Moos, 1975). Analysis revealed that the majority of participants reported they were satisfied with the program (see Table 4).

The Hillsborough County Sheriff's Office Substance Abuse Treatment Program in Tampa, Florida was evaluated in the early 1990s (Peters, Kearns, Murrin, Dolente, \& May, 1993). Several pre- and postprogram questionnaires were administered to offenders participating in the program to analyze change in specific areas: (1) knowledge for managing high-risk situations as well as knowledge in key areas of the program curriculum [Problem Situation Inventory (PSI); Hawkins et al., 1986]; (2) knowledge related to relapse prevention skills, drug education, and recovery issues [Substance Abuse Test (SAT); Peter et al., 1993]; and (3) confidence of the offender to cope successfully in 
substance taking situations [Situational Confidence Questionnaire (SCQ); Annis \& Graham, 1988]. In addition, an evaluation survey was administered prior to discharge from the program to examine satisfaction with the various components of the program and perceived usefulness of skills developed over the course of the program [Participant Evaluation Survey (PES); Peters et al., 1993]. Analyses revealed that knowledge and confidence increased from pretest to posttest among program participants (see Table 4). Furthermore, a high degree of client satisfaction with the program content, program staff, and skills attained was observed.

In an early evaluation of the Amity program by Wexler and Graham in 1993 (as cited in Graham \& Wexler, 1997) progress during treatment was assessed in addition to recidivism. Progress during treatment was measured using a self-report assessment after 3 and 6 months of program participation. The assessment addressed a variety of psychological factors including: motivation, self-esteem, self-control, anxiety, and depression. Results revealed significant improvements in all areas (see Table 4).

Another community-based substance abuse program that has been evaluated is CHOICES, which is delivered to federal inmates in Canada who are released to the community. As part of the comprehensive program evaluation completed by $\mathrm{T}^{3}$ Associates (1999c), CHOICES participants were examined. Program performance was studied using a truncated version of the modified OSAPP battery. This battery did not include knowledge measures on assertiveness or communication.

Analyses revealed that offenders who completed the intensive phase of the CHOICES program significantly increased their knowledge in a variety of areas related to substance use (see Table 4). These results suggest that the program was able to 
increase knowledge about the effects and consequences of substance use and knowledge of essential skills for changing substance-abusing behaviour. Additional analyses revealed no significant differences between offenders who completed the pre- and posttesting and offenders who did not. Accordingly, it could be concluded with some certainty that if offenders who did not complete the battery had provided this information, their scores on knowledge would be the same as the group who completed the tests $\left(\mathrm{T}^{3}\right.$ Associates Inc., 1999c).

Table 4 summarizes the findings from the second-generation substance abuse program evaluation literature. This summary illustrates efforts to advance correctional substance abuse program evaluation through the measurement of additional program outcomes, such as knowledge, confidence, motivation, and client satisfaction. From the literature, substantial evidence suggests that substance abuse programming increases offender knowledge. Specific areas that have demonstrated knowledge increases are: (1) communication, (2) decision making, (3) assertiveness, (4) consequences of alcohol and drugs, (5) problem-solving, (6) high-risk situations and relapse, and (7) coping. Confidence to manage relapse and motivation also seem to be positively influenced by treatment. Furthermore, offenders who participate in substance abuse treatment appear to be satisfied with the intervention and program staff.

This chapter detailed both the first- and second-generation evaluation literature. Examination of first-generation evaluations demonstrates the effectiveness of correctional substance abuse programming in reducing recidivism and relapse. The movement from first- to second-generation evaluation provides additional outcome information that illustrates the various effects that programming can have on substance-abusing offenders 
besides reduced recidivism and relapse. Evidence from second-generation evaluations tends to support the ability of this treatment to increase knowledge, confidence, and motivation. Summarized in the next chapter are the limitations of this body of evaluation research to provide the context for moving the evaluation of correctional communitybased substance abuse programs further into the second-generation. 
Chapter 5: Summary of Literature Limitations, Hypotheses, and Evaluation Issues

Review of the first- and second-generation substance abuse program evaluation literature highlighted several limitations. First, within this body of literature, the TC is the most widely studied substance abuse treatment approach. As a result, little is known about the effectiveness of other substance abuse treatment models used with offender populations. In addition, the majority of the evaluations assessed institutionally-based programs. With limited focus on community-based substance abuse programs, minimal evidence exists on the efficacy of substance abuse treatment provided to offenders who are released in the community.

Second, first-generation evaluations have been summative in nature and focused on the outcomes of recidivism and relapse. Second-generation evaluation has expanded this scope, but replication of these additional outcomes is scarce with the exception of knowledge gain. Replication of outcomes, such as motivation and confidence would lend support to previous findings.

A third issue emerging from review of the first- and second-generation evaluation literature is that the methodological designs do not appear to be based on program theory. With the first-generation literature, the interest in reduced recidivism and relapse rates (i.e., ultimate program goals) does not demand reliance on the program logic to guide the evaluation design. Consequently, many program outcomes remain unexplored. On the other hand, second-generation evaluations have investigated additional program outcomes, but the selection of outcomes does not appear to be linked to the program theory. This lack of consideration for program theory has resulted in minimal or nonexistent formative evaluation within the first- and second-generation literature. The 
inclusion of formative evaluation issues into an evaluation provides insight on how program success is achieved instead of just whether success is achieved. Furthermore, designing a program evaluation based on the program theory is critical for deciphering effects of specific program activities as well as determining whether intended short- and long-term outcomes are attained. This approach to designing evaluative research is especially beneficial when a multimodal treatment program is evaluated.

The fourth limitation of the overall evaluation research is the failure to explicitly recognize the evaluative utility of the principles of effective correctional treatment. Within this literature, it appears as though no attempt has been made to incorporate these principles into evaluations. The commonly accepted principles of effective correctional treatment can be applied to study formative evaluation issues, such as design and delivery. As with the application of program theory, the principles of effective correctional treatment could clarify how a substance abuse program achieves success.

A fifth shortcoming is specific to the second-generation evaluations; that is, the limited use of experimental designs with control groups. ${ }^{18}$ Without a randomly assigned control group, alternative factors that may account for the results in an experiment cannot be ruled out. In addition, very few second-generation evaluations incorporated comparative analyses between offenders who completed programming and those who did not. Using participants who do not complete programming as a comparison group can provide information on potential similarities and differences between program completers

\footnotetext{
18 A control group can be defined as a group formed through the random assignment of participants (Rossi et al., 1999). Random assignment involves randomly allocating participants to various treatment conditions (e.g., the treatment group and the no treatment control group). The experimental design utilizes treatment conditions that are randomly assigned (Ray, 1992).
} 
and noncompleters. ${ }^{19}$ Any discovered differences may highlight areas for program improvement that could encourage continued participation among offenders who are not likely to complete programming. These analyses could also discriminate whether program completion is necessary for visible change on various program outcomes.

Finally, from a review of the second-generation literature, it is evident that the capacity gained by program participants following treatment has not been examined. This generation has done considerable work to determine whether participants acquire knowledge related to various substance abuse issues and knowledge of skills for managing relapse. However, it would be of value to know whether participants can actually translate this knowledge into action.

As previously mentioned, the logic model outlines the MDH program theory (see Appendix A). This delineation of theory can serve as a visual representation of the hypotheses for this evaluation as well as facilitate their development. Thus, after considering the reviewed literature and consulting the logic model, several summative and formative evaluation hypotheses were generated. These hypotheses are listed below.

\section{Summative Evaluation Hypotheses}

1. Offenders who complete the program will have a greater increase in their knowledge of various prosocial skills in comparison to offenders who do not complete the program.

\footnotetext{
19 A comparison group can be conceptualized as a group of individuals who are not randomly assigned to the group, which can be compared to a treatment group that was not randomly assigned. The quasiexperimental design utilizes treatment conditions that are formed by procedures other than random assignment (Ray, 1992; Rossi et al., 1999).
} 
2. Offenders who complete the program will have a greater increase in their knowledge of various relapse prevention skills in comparison to offenders who do not complete the program.

3. Offenders who complete the program will have a greater increase in their ability on various prosocial skills in comparison to offenders who do not complete the program.

4. Offenders who complete the program will have a greater increase in their ability on various relapse prevention skills in comparison to offenders who do not complete the program.

5. Offenders who complete the program will have a greater increase in their confidence to not use substances in comparison to offenders who do not complete the program.

\section{Formative Evaluation Hypotheses}

6. Following participation in the drug and alcohol education activity, knowledge of substance abuse issues will increase.

7. Following participation in the initial counselling activity, motivation to participate in treatment will increase and motivation to change will move across the stages.

8. Following participation in the cognitive-behavioural activity, knowledge of various prosocial skills will increase.

9. Following participation in the cognitive-behavioural activity, knowledge of various relapse prevention skills will increase.

10. Following participation in the cognitive-behavioural activity, ability with various prosocial skills will increase. 
11. Following participation in the cognitive-behavioural activity, ability with various relapse prevention skills will increase.

12. Participants will perceive more change following the cognitive-behavioural activity than with the initial counselling activity or the drug and alcohol education activity.

In addition to the summative and formative hypotheses, this evaluation incorporated additional formative evaluation issues into its methodology in an effort to examine the design and delivery of the MDH program. Design and delivery issues have been interpreted as program implementation, and the principles of effective correctional treatment have been seen as a method for assessing the quality of program implementation (Gendreau et al., 1999, 2001). Therefore, these principles were applied to the assessment of MDH's program design and delivery. The formative issues related to program implementation based on the principles of effective correctional treatment are summarized below.

Formative Issues Related to Program Implementation

1. Does the program incorporate the risk principle?

2. Does the program incorporate the need principle?

3. Does the program incorporate the responsivity principle?

4. Does the program incorporate the principle of multimodal programming?

5. Does the program incorporate the principle of community programming?

6. Does the program incorporate the principle of continuity of care?

7. Does the program incorporate the principle of treatment length? 
8. Does the program incorporate the principle of program integrity?

The present second-generation evaluation of MDH attempted to address the limitations of the literature that were outlined and by doing so, move correctional community-based substance abuse program evaluation further into the second generation. The current second-generation evaluation examined program outcomes other than recidivism and relapse by taking into consideration the program's theory. Basing the evaluation design on the program's logic facilitated inspection of additional summative outcomes as well as formative outcomes, including the individual program components within the MDH program. By considering the program theory, outcomes similar to those in the second-generation literature were examined. This focus allowed for the replication of some past findings. Additionally, the principles of effective correctional treatment were included in the methodology. Investigation of the program's adherence to these principles permitted the assessment of program implementation.

In addition, a comparison group was incorporated into the design of this evaluation. The comparison group consisted of offenders who did not complete the MDH program, and comparative analyses were conducted on offenders who completed the program and offenders who did not. The comparison group was constructed in this way because all of the offenders who reside at the MDH half-way house participate in the program. Nonetheless, it is important to note that even without a control group, this evaluation design does lend itself to accurate conclusions for several reasons: (1) the characteristics of the outcomes being measured (i.e., motivation, confidence, knowledge, and ability); (2) the measurement of short-term objectives; and (3) the in-house design of the program. 
First, motivation, confidence, knowledge, and ability are quite specific to the MDH program. Consequently, it could be assumed that program participants were not likely to gain motivation, confidence, knowledge, and ability outside the program. Second, most of the outcomes measured by the pre- and posttests within the present evaluation were short-term objectives (i.e., increased knowledge of substance abuse issues and increased motivation - see Appendix A), which are highly unlikely to change without intervention. Evaluators have recommended that designs without a control group be implemented for short-term impact assessments of programs attempting to affect conditions that are unlikely to change much on their own (Rossi et al., 1999). Although this evaluation used a pre- and posttest design to measure some of the long-term objectives, such as ability with prosocial and relapse prevention skills (see Appendix A), this design can be considered appropriate because these outcomes are not likely to change without treatment. Finally, conclusions that are more accurate can be drawn from the analyses because of the residential nature of this program. Given that the program is residential, the clients are always in the program; therefore, little else would have influenced their knowledge, ability, confidence, and motivation related to substance abuse. 
Design

This evaluation can be conceptualized as a 2-group quasi-experimental pretestposttest design consisting of a treatment group (offenders who completed the MDH substance abuse program) and a comparison group (offenders who did not complete the MDH program). Thus, the independent variable (IV) consists of two levels: a full intervention (treatment group) and a partial intervention (comparison group). A variety of instruments were administered to measure the dependent variables (DVs). The DVs include: knowledge and ability with assertiveness, anger, and problem-solving (i.e., prosocial skills), knowledge and ability with developing a relapse prevention plan, identifying high-risk situations, and using relapse prevention techniques (i.e., relapse prevention skills), knowledge of substance abuse issues, motivation, and confidence. These measures were administered at program intake (pretest) and program completion or departure (posttest). ${ }^{20}$

Participants

Offenders. From February 24, 2003 to June 14, 2004, 103 male offenders on parole from federal institutions participated in the $\mathrm{MDH}$ substance abuse treatment program. Over this 16-month period, data were collected on 82 offenders. Seventeen

\footnotetext{
20 It should be noted that the RCQ-TV was administered at three different times over the course of the program (i.e., at intake, after the initial counselling activity, and at program completion or departure). The administration of this instrument after the initial counselling activity facilitates examination of the specific hypothesis related to increased motivation due to the initial counselling activity. The video questionnaires adhere to the concept of the pre- and posttest design, but were not administered at the beginning and the end of the overall MDH program. Given that these questionnaires were designed to measure change in knowledge following the drug and alcohol education activity, the questionnaires are administered before and after this specific program activity.
} 
offenders, residing at the half-way house during this period of time were readmissions to the program. Thus, these offenders were not included in the sample, given they had previously participated in the substance abuse program. In addition, five offenders refused to take part in the evaluation. Of the 82 participants, 48 completed the 8 -week program (58.5\%) and 34 did not (41.5\%). The 34 offenders who did not complete the program ranged in program involvement from less than one week to seven weeks, with an average involvement of 3.53 weeks $(S D=2.60)$.

Program staff. During the 16 months of data collection, three program staff were responsible for program delivery. Program delivery included one-on-one counselling and the facilitation of cognitive-behavioural activity. These specific staff members completed the pre- and postprogram staff survey on the motivation, knowledge, and ability of each offender they counselled.

\section{Materials}

Prior to this evaluation, $\mathrm{MDH}$ administered a series of questionnaires at program intake and program completion or departure to program participants. Given that MDH had instituted an ongoing data collection process, applicable data from this collection was utilized in the present evaluation. However, a few constructs were of interest in the current evaluation that were not being captured by MDH. Consequently, existing objective questionnaires and newly designed questionnaires were incorporated into the program's data collection procedure.

Drug-Taking Confidence Questionnaire. The Drug-Taking Confidence Questionnaire (DTCQ; Annis \& Martin, 1985; see Appendix C) is a 50-item self-report questionnaire designed to assess self-efficacy (i.e., confidence) in a person's ability to 
resist the urge to drink heavily or use other drugs in different types of situations. A DTCQ exists for alcohol use and another for drug use. The DTCQ for alcohol use is in Appendix C. The drug use DTCQ consists of a simple substitution of the problem area (i.e., alcohol use is changed to drug use), which is reflected in Appendix C.

The questionnaire is based on Bandura's (1977) theory of self-efficacy, in which self-efficacy is defined as the belief of an individual that he or she has the ability to cope effectively with a particular high-risk situation (Annis et al., 1997). To remain consistent with this notion of high-risk, the DTCQ consists of eight categories of relapse precipitants identified by Marlatt (1985). The eight categories of high-risk situations are: (1) unpleasant emotions, (2) physical discomfort, (3) conflict with others, (4) pleasant times with others, (5) pleasant emotions, (6) urges/temptations, (7) social pressure to use, and (8) testing personal control. This eight-subscale model was supported by confirmatory factor analysis (Annis et al., 1997). The next best model to fit the data was a three-factor model consisting of three subscales: (1) negative situations, (2) positive situations, and (3) temptation situations (Annis et al., 1997).

Respondents indicate their confidence with specific situations by circling one of six response options $(0=0 \%$ or not at all confident, $20=20 \%$ confident, $40=40 \%$ confident, $60=60 \%$ confident, $80=80 \%$ confident, $100=100 \%$ or very confident). Given these response options, the subscale confidence scores can range from $0 \%$ to $100 \%$, with $0 \%-20 \%$ indicating that an offender has no or very little confidence that he will be able to resist the urge to drink or use drugs in a particular situation, and $80 \%$ $100 \%$ indicating very high confidence in his ability to resist. The desired change in 
direction from pretest to posttest is a higher percentage of confidence in the various substance-taking situations.

Coefficient alphas reveal substantial internal consistency for all eight categories of the DTCQ ranging from .79 to .96 (Annis et al., 1997). Convergent validity has been established by correlating subscale scores with consumption variables, social context of use, and other substance-abuse related variables, such as motivation to quit using (Annis et al., 1997). For example, validity analyses revealed a significant positive association between motivation and confidence in being able to quit using substances and all the subscales, substantiating that the DTCQ captures the concept of self-efficacy (Annis et al., 1997).

Readiness to Change Questionnaire - Treatment Version. The Readiness to Change Questionnaire - Treatment Version (RCQ-TV; Heather, Luce, Peck, Dunbar, \& James, 1999) was added to MDH's data collection procedure to measure motivation to change substance-abusing behaviour. The RCQ-TV was adapted from the original 30item Readiness to Change Questionnaire (RCQ; Rollnick, Heather, Gold, \& Hall, 1992). The original RCQ was intended for use with harmful drinkers not seeking treatment; however, the instrument was often used with individuals undergoing substance abuse treatment. Hence, the researchers formulated an alternative version of the RCQ that could be used with people involved in the treatment process.

As discussed in chapter 2, the transtheoretical model suggests that behaviour change is a process, and individuals who either are changing their behaviour, on their own or while participating in a program, progress through five stages of change: precontemplation, contemplation, preparation, action, and maintenance. The original 
RCQ is based on this model. However, after a principal components analysis of the $30-$ item RCQ revealed that the maintenance stage did not form a separate component and the preparation stage showed high loadings on several components, these two stages were eliminated from the factor set. Principal components analysis on the remaining items showed a clear factor structure with the three stages. As a result, the RCQ-TV is a 15item self-report instrument that locates the respondent in one of three stages of change: (1) precontemplation, (2) contemplation, and (3) action.

Each of the three stages of change is measured by five statements, which are rated on a 5-point Likert scale ranging from 1 (strongly agree) to 5 (strongly disagree) with a mid-point of "undecided". Scoring for the RCQ-TV involves coding individual responses: strongly disagree $=-2$, disagree $=-1$, undecided $=0$, agree $=1$, and strongly agree $=2$. Scores for each stage are summed and the highest score determines the stage that the offender is in. Movement through the stages is evident when the identified pretest stage of change has a lower score at posttest than the next stage in the continuum.

Each subscale of the RCQ-TV had adequate internal consistency (Cronbach's $\alpha$ ranged from .60 to .77 ). The test-retest reliability was also acceptable with correlations between the scale scores on two occasions of administration ranging from .69 to .86 . Examination of the relationships between stage of change and other relevant variables as well as a comparison of RCQ-TV scores and URICA scores established concurrent validity (Heather et al., 1999). ${ }^{21}$

\footnotetext{
21 The University of Rhode Island Change Assessment Scale (URICA), like the RCQ-TV, measures readiness to change. This instrument corresponds to four stages of change: precontemplation, contemplation, action, and maintenance.
} 
The RCQ-TV assesses motivation for changing drinking behaviour. In the current evaluation, a version of the RCQ-TV that measures motivation for changing drug taking behaviour (RCQ-TV-DU; see Appendix E) was utilized along with the alcohol version (RCQ-TV-AU; see Appendix D). The RCQ-TV-DU consists of a simple substitution of the problem area (i.e., alcohol use was changed to drug use). No psychometric properties are available for this modification, although this drug version has been used in previous research (Vanderburg, 2002).

Problem-Solving Questionnaire. The Problem-Solving Questionnaire (PSQ; D'Zurilla \& Nezu, 1990; see Appendix F) is a 20-item instrument that measures the strengths and weaknesses of an individual across various problem-solving components. The PSQ is part of a larger inventory referred to as the Social Problem-Solving Inventory. Nonetheless, the PSQ can be used on its own. Currently, the Correctional Service of Canada includes the PSQ as part of a battery of tests used to assess performance in the CHOICES community-based substance abuse program.

The PSQ consists of a series of statements that describe the way a person may think, feel, and behave when faced with a problem in everyday life. Each statement is rated by the offender on a 5-point Likert scale from 0 (not at all true of me) to 4 (extremely true of me). The total score is equal to 80 . Low total scores indicate a higher need for developing knowledge of problem-solving skills. The desired change in direction for postprogram scores is an increase in the total score.

The PSQ has high internal consistency ( $\alpha=.92$; D'Zurilla \& Nezu, 1990). T 3 Associates (1999b) found the PSQ had high internal consistency at both pretest and 
posttest ( $\alpha=.94$ and $\alpha=.95$, respectively). In addition, the test-retest reliability was acceptable, with a coefficient of .88 (D'Zurilla \& Nezu, 1990).

Several tests of validity have been conducted by D'Zurilla \& Nezu (1990). The PSQ was compared to other self-report instruments designed to measure particular aspects of problem-solving. Results revealed a significant degree of commonality between the PSQ and these measures, but not enough to make the PSQ redundant. Comparisons between the PSQ and several measures designed to reflect dissimilar constructs, such as general intelligence, showed that the PSQ was not related. As well, predictive validity was demonstrated by correlations between problem-solving abilities and competence or positive adaptation.

Assertiveness Questionnaire. The Assertiveness Questionnaire (ASQ; Correctional Service of Canada, 1993; see Appendix G) examines the ability of an offender to recognize assertive responses to situations involving substance use. This questionnaire describes nine situations in which a person is faced with either having to respond to someone who is drinking, taking drugs, or offering them alcohol or drugs. Each situation has four response options, three of which are possible assertive responses, and one is circled if the offender feels no assertive response is presented as a response option. The maximum total score of nine indicates a high level of knowledge of assertive responses to situations involving substance use.

The internal consistency of this questionnaire has been measured slightly below the minimum acceptable level of .60 , with a pre- and posttest Cronbach's alpha of $.56\left(\mathrm{~T}^{3}\right.$ Associates Inc., 1999b). Unlike an instrument based on a Likert scale, in which a respondent can chose from a continuum of response options, the ASQ only allows for a 
dichotomous response. That is, a respondent can only select a correct response or an incorrect response. Consequently, it cannot be assumed that this type of dichotomous measure would have a high level of internal consistency in comparison to an instrument using a Likert scale ( $\mathrm{T}^{3}$ Associates Inc., 1999b). The Correctional Service of Canada includes this questionnaire as part of a battery of tests to assess performance in both the OSAPP and CHOICES substance abuse programs.

Video questionnaires. Four video questionnaires were created for this evaluation to measure change in knowledge of substance abuse issues after the drug and alcohol education program activity. Each questionnaire corresponds to one of the four mandatory educational videos. The four questionnaires are: (1) the Shame Video Questionnaire (see Appendix H), (2) the Anger Video Questionnaire (see Appendix I), (3) the Denial Video Questionnaire (see Appendix J), and (4) the My Father's Son Video Questionnaire (see Appendix K).

The questionnaires are based on a 3-point scale of "true", "false", and "don't know". Each questionnaire consists of ten questions, with the exception of My Father's Son, which has five questions. The total score for each questionnaire is 10 ; however, My Father's Son has a total score of five. The total score for all four questionnaires is calculated by summing the number of correct answers. The desired change in direction for scores at posttest is an increase, indicating knowledge gain related to specific substance abuse issues.

Pre-and postprogram client surveys. The pre- and postprogram client surveys are a revised version of MDH's client satisfaction questionnaire. The original client satisfaction questionnaire was revamped according to a pretest and posttest design to 
collect additional information on the participants' satisfaction with various elements of the program. Given the suggestion that self-ratings are a useful measure of progress in treatment settings (Sia et al., 2000), the postprogram client survey was specifically redesigned to tap client perceptions of change as a result of participation in the program.

The preprogram client survey is based on a 5-point Likert scale and consists of 19 major questions (see Appendix L). The first 7 items relate to the offender's motivation, knowledge, ability, and confidence. The last 12 items relate to the offender's physical and emotional health, family and friends, and leisure activities. It should be noted that not all of the questions in the preprogram client survey were applicable to the current evaluation. Therefore, only those questions relevant to the evaluation were analyzed. The preprogram survey also collects descriptive information on the offender, such as age, educational level, learning disabilities, risk and need level, level of substance abuse severity, and participation in previous substance abuse treatment programs. This information was obtained through review of the offender's correctional file and recorded on the preprogram survey by the counsellor.

The postprogram client survey (see Appendix M) is similar to the preprogram survey, except that the offender is asked about his satisfaction with various components of the program, such as content and delivery. Included in this 33-item survey are 19 questions related to program satisfaction as well as the offender's perception of specific program activities and their impact on motivation, knowledge, and ability. The remaining 14 items deal with the offender's physical and emotional health, family and friends, and leisure activities. As with the preprogram survey, not all of the questions in the 
postprogram client survey were applicable to the present evaluation. Therefore, only those questions relevant to the evaluation were analyzed.

Pilot testing of the revised survey was done prior to the commencement of data collection for the current evaluation. Preliminary analysis illustrated that offender responses were spread across the response options on the preprogram survey. Response options on the postprogram survey tended to cluster around the higher end of the scale. Frequency tests demonstrated negatively skewed distributions on the majority of the preprogram survey items. For selected postprogram survey items, frequency tests indicated both negatively skewed distributions as well as normal distributions.

Pearson's skewness coefficient (Munro \& Page, 1993; Runyon \& Haber, 1991) was used to calculate indices of skewness to determine whether these negatively skewed distributions were in an acceptable range. For most practical applications, a skewness index of \pm 0.50 may be considered sufficiently symmetrical for analyses (Runyon \& Haber, 1991). Indices of skewness ranging from .04 to .40 were calculated for the preprogram survey. Pearson's skewness coefficients were also computed for the postprogram survey revealing a range of .23 to .82 . Although some of the indices for the postprogram survey fall outside of \pm 0.50 , these more skewed distributions can be expected given the proposed hypotheses suggest postprogram scores will increase after treatment completion.

As a self-report instrument, the reliability and validity of the client surveys completed by offenders may be viewed as suspect. However, two systematic analyses on the validity of substance-abusing offender self-report data and the validity of the CLAI system have been completed by the CSC that provide support for the conclusion that 
substance-abusing offender self-report data is reliable and valid. Recall from chapter 1 that the CLAI is an intake assessment done on all offenders within the federal system. The assessment is based on self-report.

A study by Robinson and Millson (as cited in Weekes et al., 1997) examined the CLAI and offender institutional files to compare their capacity in identifying substance abuse problems. Institutional files on 100 offenders who had completed the CLAI were reviewed. The CLAI and the file information corresponded in $75 \%$ of the cases. Substance abuse problems were identified by the CLAI in $20 \%$ of the cases where the files did not. Only $5 \%$ of the offender files contain information about alcohol and/or drug use, when the CLAI did not identify the individual as having a substance abuse problem.

The second study found a $72 \%$ concordance rate between the scores on the Alcohol Dependence Scale and Drug Abuse Screening Test measured in the CLAI and a personal interview (Beal, Weekes, Millson, \& Eno, 1996). Close examination of the raw scores from these two assessments revealed that the majority of scores increased by just one or two points. Significant change occurred in only $5 \%$ of cases. That is, there were few substantive changes in severity levels (e.g., no problem to intermediate problem).

Balanced Inventory of Desirable Responding. The Balanced Inventory of Desirable Responding - Version 6 (BIDR; Paulhus, 1991; see Appendix N) is a standardized assessment instrument that measures an individual's tendency to give socially desirable responses on self-report instruments (Paulhus, 1998). The BIDR consists of two subscales: self-deceptive enhancement (SDE) and impression management (IM; Paulhus, 1984, 1991, 1998). High SDE scores reflect positive selfesteem but potentially poor interpersonal adjustment (i.e., lack of insight; Paulhus, 1998). 
Individuals with high SDE scores display overconfidence (Paulhus, 1998). The IM subscale assesses an individual's tendency to give inflated self-descriptions. High IM scores reflect conscious distortion (i.e., faking or lying; Paulhus, 1998). Currently, the Correctional Service of Canada uses the BIDR in OSAPP.

The BIDR consists of 40 statements that respondents are asked to rate on a 7point Likert scale ranging from 1 (not true) to 7 (very true). Each subscale consists of 20 statements. The scale is scored by allocating one point to every question rated an extreme response (i.e., 6 or 7). Several statements are negatively keyed and need to be reverse coded. Specifically, this means that for negatively keyed items, one point is given for a response of 1 or 2 , as opposed to 6 or 7 . The total score is the sum of all the points.

In a psychometric study of the BIDR with an offender sample, Kroner and Weekes (1996) reported the following scores for offenders who completed the BIDR at intake: the SDE subscale produced a mean score of $6.04(S D=1.86)$, and the IM subscale had a mean score of $5.05(S D=1.11)$. Offenders who completed the BIDR prior to release demonstrated similar scores. These researchers also found acceptable internal consistency for the SDE and IM subscales ( $\alpha=.73$ and .84 , respectively). Other research has established convergent validity and discriminant validity (Paulhus, 1991, 1998).

Pre-and postprogram staff survey. The survey was developed to obtain information about the offenders participating in the MDH program. However, this survey is intended to capture perceptions of offender change based on the professional opinion of the program staff (i.e., counsellors). The preprogram staff survey consists of three questions: two are related to motivation and one assesses confidence (see Appendix $\mathrm{O}$ ). The postprogram staff survey is made up of five questions: three are the same as the 
preprogram staff survey examining motivation and confidence, and the other two relate to knowledge and ability on prosocial and relapse prevention skills (see Appendix O). Most of the questions are based on a 5-point Likert scale.

Key informant interviews. The author developed two key informant interview protocols. The underpinning of the key informant interview with the Executive Director was the formative issues outlined in chapter 5, and as a result, the interview covered issues related to program implementation (see Appendix P). This interview consisted of eight questions. The interview was intended to be a succinct assessment tool for collecting descriptive data to make an estimate about the program's adherence to the principles of effective correctional treatment. The key informant interview used with individual counsellors consisted of six open-ended questions related to the reading and comprehension levels of offenders (see Appendix Q).

\section{Procedure}

Prior to the initiation of data collection, the author of this evaluation delivered a workshop to the staff at MDH. The purpose of the workshop was twofold: to introduce staff to the concept of program evaluation, as most were unfamiliar with the discipline, and to review the evaluation framework that would be implemented at $\mathrm{MDH}$. At a later date, but also prior to data collection, the author provided a training session to the MDH program staff, intake officer, and Executive Director on the administration and scoring of the supplementary evaluation instruments. At this time, it was decided that all completed assessments would be scored and filed by the intake officer. During this training session, the consent and debriefing forms were reviewed. With respect to soliciting consent from offenders, staff were asked to emphasize confidentiality, anonymity, and the fact that the 
offender's participation would assist the half-way house in making the program better for offenders.

A quality control process was incorporated into data collection. This monitoring practice made certain the evaluation assessments were administered at the appropriate times and that assessments requiring scoring were scored correctly. In addition, the practice provided the author with continual involvement in the data collection. The quality control was done by the author on a weekly basis over the 16-month collection period, and consisted of two parts. The first part required that the author visit the halfway house and review all the testing completed that week. Any potential omissions or errors were discussed and resolved with the appropriate counsellor and the intake officer. The second part of the monitoring process involved reviewing a weekly roster faxed to the author. Appraisal of the roster allowed for monitoring movement in and out of the program. Specifically, the roster outlined the offenders who were starting the program, finishing the program, or leaving the program.

When an offender arrived at $\mathrm{MDH}$, he was assigned a program counsellor and by day two the offender would meet with his counsellor. At this point, the intake process was initiated. This process began with the counsellor using the consent form (see Appendix R) to explain that $\mathrm{MDH}$ was undergoing an evaluation of its treatment program, in an effort to improve the program and ensure it was meeting the needs of program participants. After this initial briefing, the counsellor asked the offender if he would volunteer to participate in the evaluation. When an offender agreed to participate, he signed the consent form. With consent attained, the counsellor administered the measures specific to the evaluation (i.e., RCQ-TV and BIDR) as well as those 
instruments already included as part of the MDH intake process (i.e., DTCQ, PSQ, ASQ, and preprogram client survey). The intake instruments were not provided to the offender in any particular order.

It should be noted that five offenders did not consent to participate in the evaluation. In these instances, the measures specific to the evaluation were not administered. However, instruments germane to the $\mathrm{MDH}$ intake process were mandatory for them to complete. None of the mandatory testing done by these offenders was included in the evaluation database. For data entry purposes, all offenders who participated in the evaluation were assigned an identification number so the data could not be associated with their names.

Offenders were given a day to respond to all of the intake assessments; however, on average it took offenders about 2 to 3 hours to complete the intake battery. While completing the questionnaires, the offenders were able to meet with their counsellor to get clarification and/or guidance, if required. For offenders with below grade eight education and/or reading difficulties, of which there were only a few, the assigned counsellor sat with the offender to assist with the completion of the entire battery. This assistance included: reading questions, paraphrasing questions, and/or explaining concepts. For all offenders, counsellors ensured every question on each assessment was completed. In general, counsellors found that offenders were able to read the battery of questionnaires and understand the content of the questions and thus, respond accordingly.

By day three, offenders watched the four mandatory videos, when the drug and alcohol education component of the program was being delivered. Before watching the videos, the intake officer gave the offender the prevideo questionnaires to complete. By 
the end of the first week at MDH (i.e., the end of the initial counselling activity), the counsellor reviewed the completed intake assessments with the offender. At this time, the counsellor provided the offender with the postvideo questionnaires to complete, as well as another RCQ-TV and BIDR. As with the intake, these instruments were not given to the offender in any particular sequence. In addition, the counsellor used this time to fill out the preprogram staff survey.

Once an offender completed the program, he was given the debriefing form to read, and the counsellor answered any questions he had about the evaluation (see Appendix S). At this time, he was also given MDH's posttesting (i.e., DTCQ, PSQ, ASQ, and postprogram client survey) as well as the instruments specific to the evaluation (i.e., RCQ-TV and BIDR) in a random order. Similar to the intake process, offenders were given a day to complete the posttesting. However, on average it took them 1 hour to complete the battery. Counsellors reviewed the posttesting results with all program completers. At the end of program participation or departure from the program, the counsellor completed the postprogram staff survey.

When an offender did not complete the program, most often the circumstances did not permit administration of posttesting prior to his departure from the half-way house. The large majority of offenders who departed early were detained at the Ottawa-Carleton Detention Center (OCDC) before to being transferred back to the institution they were paroled from. Therefore, the author made arrangements with a discharge planner at OCDC to administer posttesting.

When an offender left MDH prior to program completion, the author contacted the discharge planner to inform the planner that an offender from $\mathrm{MDH}$ had been sent to 
OCDC. After receiving this notification, the discharge planner visited the offender to request he complete the posttesting. If the offender agreed, the planner provided him with the posttesting battery. The discharge planner collected the completed posttesting a few days later. When an offender did not complete the posttesting at OCDC, the author mailed a posttesting package to the offender at the institution he was transferred to. Packages were mailed to noncompleters, who did not do the testing, at least two times after their departure from OCDC.

Key informant interviews were conducted with the Executive Director and two counsellors. These interviews were booked and lead by the author. The interviews were conducted in the office of each key informant. The interview with the Executive Director took 40 minutes to complete. On average, the interviews with the counsellors lasted 20 minutes.

The actual interview followed a suggested three-stage structure consisting of: a warm-up phase, the interview phase, and a cool-down phase (W. Thorngate, personal communication, March 3, 2004). Therefore, to start each key informant interview, the author did a warm-up by engaging in some small talk, such as thanking the informant for sharing some of his or her time, and asking how things were going at the half-way house. Once the dialogue appeared to be relaxed, each question was read aloud and expanded upon to provide additional detail. Key informants were able to spend as much time as they felt necessary to respond to individual questions. When responses were unclear or required clarification, the author would probe the key informant for additional information. While key informants were answering questions, the author took notes. After the interview questions were posed, the cool-off phase of the interview 
commenced. During the cool-off, informants were asked if they wanted to add anything to the interview and if he or she had any questions.

An issues and indicators matrix was developed to more explicitly outline each of the hypotheses and issues (see Table 5). The issues and indicators matrix is a useful evaluative tool because it summarizes the research strategy for the evaluation of MDH. Indeed, the matrix serves as a preliminary data analysis plan. In addition to outlining key issues (i.e., issues/hypotheses), the matrix also details the measure or measures of the issues (i.e., indicators) and the methodology to complete the examination (i.e., methods). As can be seen from Table 5, this evaluation implemented three methodologies: (1) an impact assessment, (2) client and program staff surveys, and (3) key informant interviews. The following section describes these various methodologies. For each methodology, a narrative details several factors: (1) description and procedure of the method, (2) advantages of the method, and (3) summary of the information the method provided.

Impact assessment. Impact assessment is undertaken in a program evaluation to determine whether a program produces its desired effects (i.e., the program attains its program outcomes) over and above what would have occurred either with or without the program (Rossi et al., 1999). To complete an impact assessment, measurable outcomes that represent the outcomes of the program need to be identified. For the present evaluation, several measurable outcomes have been identified representing the short- and long-term outcomes of the MDH program. Given that these outcomes measure short-term and long-term program objectives, the impact assessment then, addresses issues/questions 
Table 5

Summary of Evaluation Issues, Questions, Indicators, and Methods

\begin{tabular}{lll}
\hline Issues/Questions & Indicators & Methods \\
\hline
\end{tabular}

Impact (Summative Hypotheses)

1. Is knowledge on prosocial skills increased?

2. Is knowledge on relapse prevention skills increased?

3. Is ability with prosocial skills increased?

4. Is ability with relapse prevention skills increased?

5. Is confidence increased?
- Client opinion

- Level of assertiveness

- Level of problemsolving

- Staff opinion

- Client opinion

- Staff opinion

- Client opinion

- Staff opinion

- Client opinion

- Staff opinion

- Client opinion

- Level of confidence
- Client survey

- Impact assessment

- Impact assessment

- Staff survey

- Client survey

- Staff survey

- Client survey

- Staff survey

- Client survey

- Staff survey

- Client survey

- Impact assessment

\section{Impact (Formative Hypotheses)}

6. Is motivation increased following initial counselling component?

7. Is substance abuse knowledge increased following drug and alcohol education?

8. Is knowledge of prosocial skills increased following the cognitive-behavioural activity?
- Client opinion

- Level of motivation

- Staff opinion

- Client opinion

- Level of knowledge

- Client opinion
- Client survey

- Impact assessment

- Staff survey

- Client survey

- Impact assessment

- Client survey 


Issues/Questions (Hypotheses) Indicators Methods

9. Is knowledge of relapse prevention skills increased

- Client opinion

- Client survey

following the cognitivebehavioural activity?

10. Is ability on prosocial skills increased following the cognitive-behavioural activity?

11. Is ability on relapse prevention skills increased following the cognitivebehavioural activity?

12. Is there more change following the cognitivebehavioural activity than the counselling or drug and alcohol education?

- Client opinion

- Client survey

- Client opinion

- Client survey

- Client opinion

- Client survey

Formative Issues

13. Does the program implement the following principles:

- Key informant opinion

- Risk?

- Need?

- Responsivity?

- Multimodal programming?

- Community programming?

- Continuity of care?

- Treatment length?

- Program integrity? 
in the impact section of the issues and indicators matrix (see Table 5). To assess the defined outcomes, a pre- and posttest design was selected.

A pre- and posttest design is a design in which assessment instruments are administered to program participants before and after a program (Rossi et al., 1999). The pre- and posttest design is beneficial because it facilitates the examination of changes in clients after participating in a treatment program. Changes in knowledge, ability, confidence, and motivation are examples of outcomes that can be measured with this method. The instruments used in the current impact assessment were: (1) the client survey, (2) the staff survey, (3) the RCQ-TV, (4) the DTCQ, (5) the ASQ, (6) the PSQ, and (7) the video questionnaires. Data collected from the impact assessment using the standardized instruments are presented in Appendix T.

Client and program staff surveys. In general, a survey collects information from a group of individuals by means of interviews or questionnaires (Rossi et al, 1999). The inclusion of the client survey in this evaluation is a beneficial method because it allows for the collection of data from participants that is unobservable, such as perceptions, experience, and expectations (Dagger, 2002). Of significance to the evaluation is the fact that the client survey allows for the examination of offender perceptions on the effectiveness of the program (Chan et al., 1997; Conners \& Franklin, 2000; Glider et al., 1997). Furthermore, the completed client survey contains offenders' self-ratings, which are useful measures of progress in treatment settings (Sia et al., 2000).

Offenders upon their arrival and departure from the program completed the client survey. This assessment process is part of the pre- and posttest design previously described. As with the offenders, staff can provide unique information on issues, such as 
change among clients. A staff survey was implemented to gather data on counsellors' professional opinions about offender motivation, confidence, knowledge, and ability. Specific data gathered from the pre- and postsurveys completed by the offenders and staff are outlined in Appendix T.

Key informant interview. A key informant interview can be defined as a one-onone consultation with a person who has a professional position on the nature and scope of a program, the program participants, as well as the program staff (Rossi et al., 1999). A key informant interview is a valuable methodology because the professional opinion is provided by someone who has intimate knowledge of the workings of the program, which usually cannot be obtained from any other source. In the current evaluation, this method was used to gather information on the program's adherence to the principles of effective correctional treatment as well as the reading and comprehension level of offenders. Data collected from the key informant interview with the Executive Director are presented in Appendix $T$. 


\section{Chapter 7: Results}

Data analyses were conducted using the Statistical Package for the Social Sciences, version 11.5. An alpha level of .05 was applied to all statistical tests, unless otherwise indicated. For tests of association between categorical variables, chi-square analyses were conducted except when the expected cell count was less than five. In cases where cell counts were less than five, gamma was used. For all relevant analyses, the following potential covariates were examined: age, risk level, pretest BIDR SDE scores, and pretest BIDR IM scores. Any of these four covariates were included in an analysis when the sample size was appropriate, the assumptions of analysis of covariance were met, and the error variance was reduced.

For other statistical tests conducted for this evaluation, the associated assumptions were assessed and met, unless otherwise indicated. For the examination of effect sizes, established conventions were applied. Cohen's (1992) effect size conventions for MANOVA and ANOVA were used to examine the magnitude of multivariate tests (small $=.02$, medium $=.13$, large $=.30)$ and univariate tests $($ small $=.01$, medium $=.06$, large $=$ .14), respectively. In addition, Cohen's conventions for correlations were utilized where appropriate $($ small $=.10$, medium $=.30$, large $=.50)$.

\section{Examination of Results}

When examining the results of the current evaluation, statistical significance testing was not the only approach taken into consideration. In conducting field research, large samples are often difficult to attain, as was the case in the present evaluation. Studies with small samples can lack sufficient statistical power to detect significant 
differences. Many researchers are of the opinion that a nonsignificant result does not necessarily imply that there was no treatment effect; thus, it has been suggested that effect sizes be examined to assess the magnitude of a relationship (Gendreau, 2001; Schmidt, 1996). As well, effect sizes can be studied in conjunction with significance testing to inform the interpretation of results and guide further examination of nonsignificant findings. Moderate effect sizes and potentially even small and smallmoderate effect sizes associated with nonsignificant analyses may be worth considering as these results could be of practical importance (Lösel, 2001).

Triangulation was also applied to the examination of the results. Triangulation involves using more than one source of data to support a single point (Marshall \& Rossman, 1995; Tutty, Rothery, \& Grinnell, 1996). This technique is beneficial because a research question can be corroborated or elaborated through the examination of findings from different data sources. In the current evaluation, findings are triangulated where a hypothesis was tested with offender self-report and program staff assessment. ${ }^{22}$ Researchers have suggested that if all analyses for a particular hypothesis arrive at the same outcome, the generalizability of the study is strengthened (Marshall \& Rossman, 1995; Tutty et al., 1996).

\section{Sample Descriptives}

All of the offenders who reside at $\mathrm{MDH}$ are male. The average age of offenders participating in this evaluation was 34.8 years $(S D=8.72)$. An analysis of variance using

\footnotetext{
22 In general, the corresponding knowledge and ability items on the postprogram client survey and the postprogram staff survey were significantly correlated ( $r$ ranged from .36 to .48 ) representing moderate and moderate-large effect sizes.
} 
age demonstrated that offenders who completed the program were significantly older $(M$ $=37.52, S D=9.31)$ than offenders who did not complete the program $(M=30.97, S D=$ $6.11), F(1,80)=12.88, p=.001, \eta_{p}^{2}=.14$

Statistical analyses of other demographic variables showed nonsignificant differences across the two groups (see Table 6), with the exception of risk level. Specifically, a higher percentage of high risk cases among noncompleters than completers was evident. Differences between program completers and noncompleters approach significance $(p<.10)$ for need level, with a higher percentage of medium need cases among program completers than noncompleters. ${ }^{23}$

As can be seen, just $3.7 \%$ of the entire sample had less than a grade eight education. The majority of offenders $(57.3 \%)$ had completed some high school. Learning disabilities were not commonly reported by offenders at intake, with $85.4 \%$ recorded as not having this deficit. A large percentage of this sample (64.6\%) was currently serving a sentence for crimes against the person. Almost the entire sample $(98.8 \%)$ was identified as either moderate or high need. Although there was a higher percentage (73.5\%) of high need individuals among noncompleters in comparison to completers $(54.2 \%)$, this difference only approached significance $(p=.056)$.

In addition to the aforementioned sample descriptives, the mean scores on the BIDR were examined for the sample. The mean Impression Management (IM) scores and the mean Self-Deceptive Enhancement (SDE) scores at each of the three administration

23 Risk and need levels were assessed according to CSC's accepted designation. 
Table 6

Sample Descriptives for the Total Sample and for Each Group

\begin{tabular}{|c|c|c|c|c|c|c|c|}
\hline \multirow[b]{3}{*}{ Variable } & \multirow[b]{3}{*}{$\gamma$} & & & \multicolumn{4}{|c|}{ Group } \\
\hline & & \multicolumn{2}{|c|}{ Total } & \multicolumn{2}{|c|}{$\begin{array}{l}\text { Program } \\
\text { completers }\end{array}$} & \multicolumn{2}{|c|}{ Noncompleters } \\
\hline & & $\%$ & $n$ & $\%$ & $n$ & $\%$ & $n$ \\
\hline Education level & .05 & & & & & & \\
\hline$<$ Grade 8 & & 3.7 & 3 & 2.1 & 1 & 5.9 & 2 \\
\hline Some high school & & 57.3 & 47 & 60.4 & 29 & 52.9 & 18 \\
\hline GED & & 13.4 & 11 & 8.3 & 4 & 20.6 & 7 \\
\hline$>$ High school & & 25.6 & 21 & 29.2 & 14 & 20.6 & 7 \\
\hline Learning disabilities & -.01 & & & & & & \\
\hline No & & 85.4 & 70 & 85.4 & 41 & 85.3 & 29 \\
\hline Yes & & 14.6 & 12 & 14.6 & 7 & 14.7 & 5 \\
\hline Current offence type & .24 & & & & & & \\
\hline Crime against person & & 64.6 & 53 & 60.4 & 29 & 70.6 & 24 \\
\hline Crime against property & & 28.0 & 23 & 29.2 & 14 & 26.5 & 9 \\
\hline Criminal code traffic & & 2.4 & 2 & 4.2 & 2 & 0 & 0 \\
\hline Other federal statutes & & 4.9 & 4 & 6.3 & 3 & 2.9 & 1 \\
\hline Risk level & $-.38 *$ & & & & & & \\
\hline High & & 41.5 & 34 & 33.3 & 16 & 52.9 & 18 \\
\hline Medium & & 47.6 & 39 & 52.1 & 25 & 41.2 & 14 \\
\hline Low & & 11.0 & 9 & 14.6 & 7 & 5.9 & 2 \\
\hline Need level & $-.41^{\dagger}$ & & & & & & \\
\hline High & & 62.2 & 51 & 54.2 & 26 & 73.5 & 25 \\
\hline Medium & & 36.6 & 30 & 43.8 & 21 & 26.5 & 9 \\
\hline Low & & 1.2 & 1 & 2.1 & 1 & 0 & 0 \\
\hline
\end{tabular}


times for the noncompleter group ranged from below average to average compared to offender norms (Paulhus, 1998; see Table 7). For the program completer group, the mean IM and SDE scores at each test administration time ranged from slightly above average to very much above average compared to offender norms (Paulhus, 1998; see Table 7).

A doubly multivariate repeated measures analysis of variance was conducted to determine whether program completers differed from noncompleters on the two subscales of the BIDR and whether any differences existed across the three test administration times. Using Wilks' $\lambda$, it was found that the interaction between group and test administration time was not significant on the set of dependent variables, $F(4,182)=.55$, $p=.70, \eta_{\mathrm{p}}^{2}=.01$. However, the multivariate results showed that the groups differed significantly on the linear combination of the dependent variables, $F(2,45)=7.36, p<$ $.01, \eta_{p}^{2}=.25$. Univariate results revealed that the groups differed significantly on both the IM subscale and the SDE subscale (see Table 7). Specifically, program completers seemed to be engaging in more impression management (i.e., trying to present oneself in a favourable light) and had more lack of personal insight (i.e., overconfidence in one's ability) in comparison to the noncompleter group.

The multivariate results for the within-subjects effect demonstrated a significant difference across the linear combination of test administration times, $F(4,182)=2.90, p$ $<.05, \eta_{\mathrm{p}}^{2}=.06$. Univariate results indicated significant differences across time on the SDE subscale and differences approached significance for the IM subscale, with both magnitudes being moderate. The within-subjects contrasts revealed significant trends. A quadratic trend was evident for the IM subscale, $F(1,46)=7.07, p<.05, \eta_{p}^{2}=.13$, with the IM scores decreasing from pretest to midtest and then increasing from midtest to 
Table 7

Univariate Results from MANOVA Examining the BIDR SDE and IM Subscales Over

Time as a Function of Program Completion

\begin{tabular}{|c|c|c|c|c|c|c|}
\hline Effect & & & $M$ & $S E$ & $F$ & $\eta_{p}^{2}$ \\
\hline \multicolumn{7}{|c|}{ Between-subjects effects } \\
\hline \multicolumn{7}{|l|}{ Group } \\
\hline \multirow[t]{2}{*}{ BIDR SDE } & \multicolumn{2}{|l|}{ PC } & 7.48 & .39 & $10.58^{* *}$ & .19 \\
\hline & \multicolumn{2}{|l|}{$\mathrm{NC}$} & 4.56 & .81 & & \\
\hline \multirow[t]{2}{*}{ BIDR IM } & \multicolumn{2}{|l|}{ PC } & 6.15 & .48 & $6.56^{*}$ & .13 \\
\hline & \multicolumn{2}{|l|}{$\mathrm{NC}$} & 3.33 & .99 & & \\
\hline \multicolumn{7}{|c|}{ Within-subjects effects } \\
\hline \multicolumn{7}{|l|}{ Test time } \\
\hline \multirow[t]{3}{*}{ BIDR SDE } & \multicolumn{2}{|l|}{ Pre } & 5.44 & .58 & $3.78^{*}$ & .08 \\
\hline & \multicolumn{2}{|l|}{ Mid } & 5.68 & .52 & & \\
\hline & \multicolumn{2}{|l|}{ Post } & 6.94 & .59 & & \\
\hline \multirow[t]{3}{*}{ BIDR IM } & \multicolumn{2}{|l|}{ Pre } & 4.77 & .65 & $3.12^{\dagger}$ & .06 \\
\hline & \multicolumn{2}{|l|}{ Mid } & 3.90 & .69 & & \\
\hline & \multicolumn{2}{|l|}{ Post } & 5.55 & .66 & & \\
\hline \multicolumn{7}{|c|}{ Test time $\mathrm{x}$ group } \\
\hline \multirow[t]{6}{*}{ BIDR SDE } & \multirow[t]{2}{*}{ Pre } & $\mathrm{PC}$ & 6.87 & .53 & .73 & .02 \\
\hline & & $\mathrm{NC}$ & 4.00 & .71 & & \\
\hline & \multirow[t]{2}{*}{ Mid } & $\mathrm{PC}$ & 6.79 & .47 & & \\
\hline & & $\mathrm{NC}$ & 4.56 & .78 & & \\
\hline & \multirow[t]{2}{*}{ Post } & $\mathrm{PC}$ & 8.77 & .50 & & \\
\hline & & $\mathrm{NC}$ & 5.11 & 1.12 & & \\
\hline \multirow[t]{6}{*}{ BIDR IM } & \multirow[t]{2}{*}{ Pre } & $\mathrm{PC}$ & 5.87 & .60 & .32 & .007 \\
\hline & & $\mathrm{NC}$ & 3.67 & .71 & & \\
\hline & \multirow[t]{2}{*}{ Mid } & $\mathrm{PC}$ & 5.46 & .63 & & \\
\hline & & $\mathrm{NC}$ & 2.33 & .85 & & \\
\hline & \multirow[t]{2}{*}{ Post } & $\mathrm{PC}$ & 7.10 & .59 & & \\
\hline & & $\mathrm{NC}$ & 4.00 & 1.00 & & \\
\hline
\end{tabular}

Note. Group $=$ program completers $(\mathrm{PC})$ and noncompleters $(\mathrm{NC})$; Test time $=$ intake (Pre), posteducation program component (Mid), and postprogram or departure from program (Post).

${ }^{\dagger} p=.10 .{ }^{*} p=.05 .{ }^{* *} p=.01$. 
posttest. In addition, a linear trend was evident for the SDE subscale, $F(1,46)=5.21, p$ $<.05, \eta_{p}^{2}=.10$, with the SDE scores continuing to increase from pretest to posttest.

\section{Substance Abuse Descriptives}

Statistical analyses of the substance abuse descriptives showed nonsignificant differences between program completers and noncompleters (see Table 8). Of the 82 offenders in the evaluation, $50 \%$ disclosed cocaine as the drug they used most often. Lower frequencies were reported for alcohol (18.3\%), marijuana $(12.2 \%)$, heroin $(8.5 \%)$, and other narcotics $(11.0 \%)$. Of the offenders who reported using a drug most often $(n=$ 65), a large majority (72.3\%) were identified as having either a substantial or severe drug problem. For offenders who identified alcohol as their substance of choice $(n=15)$, $26.7 \%$ were classified with either substantial or severe symptoms of dependence.

Other substance abuse variables of interest relate to previous substance abuse treatment participation (see Table 9). In an examination of previous substance abuse participation and the completion of previous substance abuse programming, for both institutional and community programs, chi-square analyses failed to reach significance (see Table 9). An overwhelming percentage of the offenders (90.2\%) reported previous participation in substance abuse treatment programs. Of the offenders who participated in previous substance abuse treatment, $90.5 \%$ reported involvement within a federal institution, whereas just $78.4 \%$ reported involvement with community-based substance abuse treatment. Almost $87 \%$ of offenders who participated in institutional substance abuse programming reported completing at least one of these programs. In comparison, fewer offenders (69\%) who participated in community-based substance abuse treatment reported completing at least one of these programs. 
Table 8

Substance Abuse Descriptives Variables for the Total Sample and for Each Group

\begin{tabular}{|c|c|c|c|c|c|c|c|}
\hline \multirow[b]{3}{*}{ Variable } & \multirow[b]{3}{*}{$\gamma$} & & & \multicolumn{4}{|c|}{ Group } \\
\hline & & \multicolumn{2}{|c|}{ Total } & \multicolumn{2}{|c|}{$\begin{array}{l}\text { Program } \\
\text { completers }\end{array}$} & \multicolumn{2}{|c|}{ Noncompleters } \\
\hline & & $\%$ & $n$ & $\%$ & $n$ & $\%$ & $n$ \\
\hline Substance of choice & -.12 & & & & & & \\
\hline Alcohol & & 18.3 & 15 & 25.0 & 12 & 8.8 & 3 \\
\hline Cocaine & & 50.0 & 41 & 41.7 & 20 & 61.8 & 21 \\
\hline Marijuana & & 12.2 & 10 & 14.6 & 7 & 8.8 & 3 \\
\hline Heroin & & 8.5 & 7 & 6.3 & 3 & 11.8 & 4 \\
\hline Other drugs & & 11.0 & 9 & 12.5 & 6 & 8.8 & 3 \\
\hline Drug abuse severity & .14 & & & & & & \\
\hline Severe and substantial & & 72.3 & 47 & 75.0 & 27 & 69.0 & 20 \\
\hline Moderate & & 15.4 & 10 & 13.9 & 5 & 17.2 & 5 \\
\hline Low and none & & 12.3 & 8 & 11.1 & 4 & 13.8 & 4 \\
\hline Alcohol abuse severity & .20 & & & & & & \\
\hline Severe and substantial & & 26.7 & 4 & 33.3 & 4 & 0.0 & 0 \\
\hline Moderate & & 33.3 & 5 & 25.0 & 3 & 66.7 & 2 \\
\hline Low and none & & 40.0 & 6 & 41.7 & 5 & 33.3 & 1 \\
\hline $\begin{array}{l}\text { MDH assessment of } \\
\text { substance abuse } \\
\text { treatment need }\end{array}$ & -.07 & & & & & & \\
\hline High & & 42.7 & 35 & 41.7 & 20 & 44.1 & 15 \\
\hline Medium & & 56.1 & 46 & 56.3 & 27 & 55.9 & 19 \\
\hline Low & & 1.2 & 1 & 2.1 & 1 & 0.0 & 0 \\
\hline
\end{tabular}


Evaluation of a Correctional Substance Abuse Program 120

Table 9

Percentages of Previous Substance Abuse Treatment Participation and Completion of

Substance Abuse Programs for the Total Sample and for Each Group

\begin{tabular}{|c|c|c|c|c|c|c|c|}
\hline \multirow[b]{3}{*}{ Variable } & & \multirow{2}{*}{\multicolumn{2}{|c|}{ Total }} & \multicolumn{4}{|c|}{ Group } \\
\hline & & & & & & Nonc & eters \\
\hline & $\chi^{2 / \gamma}$ & $\%$ & $n$ & $\%$ & $n$ & $\%$ & $n$ \\
\hline
\end{tabular}

Any previous SA

.19

treatment participation

No

Yes

Any previous institutional SA treatment participation

No

Yes

Any previous community SA treatment participation
$21.6 \quad 16$

$78.4 \quad 58$
8.3

91.7
4

44

11.8

4

$90.2 \quad 74$

$9.5 \quad 7$

6.8

3

$13.3 \quad 4$

90.5

67

93.2

41

86.7

26

.73

$\begin{array}{lcccccc}\text { No } & 21.6 & 16 & 25.0 & 11 & 16.7 & 5 \\ \text { Yes } & 78.4 & 58 & 75.0 & 33 & 83.3 & 25\end{array}$

Completion of previous $\quad .36$ institutional SA

treatment

No

$\begin{array}{cccccc}13.6 & 9 & 10.0 & 4 & 19.2 & 5 \\ 86.4 & 57 & 90.0 & 36 & 80.8 & 21\end{array}$

Yes

.51

Completion of previous community $\mathrm{SA}$ treatment

\begin{tabular}{lcccccc} 
No & 31.0 & 18 & 27.3 & 9 & 36.0 & 9 \\
Yes & 69.0 & 40 & 72.7 & 24 & 64.0 & 16 \\
\hline
\end{tabular}

Note. $\mathrm{SA}=$ substance abuse. 


\section{Changes to the Original Program}

At the beginning of September 2003, the drug and alcohol education videos were removed from the treatment process. Given this program change, analyses were conducted to determine whether the offenders who participated in the video component of the program and the offenders who did not differed significantly on various postprogram outcomes. Independent-samples $t$ tests were conducted with dependent variables that were hypothesized to be influenced by the educational video component, including motivation, knowledge and ability with problem solving, assertiveness, anger, and relapse prevention, as well as confidence with various substance taking situations.

Due to the large number of $t$ tests that were performed to compare these two groups (i.e., offenders who watched the videos and offenders who did not watch the videos), Bonferroni's correction was used with a critical alpha of .004 . No significant differences were detected between the two groups (see Table 10). Since the video component did not have an impact on postprogram outcomes, it can be suggested that offenders who watched the videos were similar to offenders who did not watch the videos.

Also modified about half way through data collection was the format for delivering the initial counselling activity and cognitive-behavioural activity. Given the change to delivery, analyses were conducted to determine whether the offenders who participated in the original delivery format and the offenders who participated in the revised format differed significantly on various postprogram outcomes. Independentsamples $t$ tests were conducted with dependent variables that may have been influenced by the counselling and cognitive-behavioural components of the program. These variables included motivation, knowledge and ability with problem solving, 
Table 10

Comparison Between Offenders who Did and Did Not Participate in the Drug and Alcohol Education Activity

\begin{tabular}{lccccc}
\hline & \multicolumn{5}{c}{ Group } \\
\cline { 2 - 5 } Instrument & Video component & No video component & \\
& $M(S D)$ & $n$ & $M(S D)$ & $n$ & $t$ \\
\hline After counselling & & & & & \\
RCQ & $3.00(.00)$ & 18 & $2.95(.22)$ & 40 & -1.43 \\
Completion/departure & & & & & \\
RCQ & $2.88(.33)$ & 25 & $3.00(.00)$ & 37 & 1.81 \\
PSQ & $58.35(14.94)$ & 26 & $59.37(13.39)$ & 35 & .28 \\
ASQ & $7.08(1.70)$ & 26 & $6.69(1.62)$ & 35 & -.91 \\
DTCQ negative situations & $92.47(11.82)$ & 24 & $94.04(12.57)$ & 37 & .49 \\
DTCQ positive situations & $94.92(7.46)$ & 24 & $92.54(19.45)$ & 37 & -.57 \\
DTCQ temptation situations & $86.67(23.32)$ & 24 & $89.66(18.92)$ & 37 & .55 \\
Client survey - ability with problem solving & $4.35(.89)$ & 34 & $4.48(.68)$ & 31 & .62 \\
Client survey - ability with assertiveness & $4.39(.72)$ & 34 & $4.38(.94)$ & 31 & -.07 \\
Client survey - knowledge of anger & $4.57(.73)$ & 23 & $4.56(.67)$ & 32 & -.01 \\
Client survey - ability with anger & $4.39(.84)$ & 34 & $4.59(.62)$ & 31 & .98 \\
Client survey - knowledge of RP skills & $13.63(2.26)$ & 24 & $13.76(1.82)$ & 33 & .25 \\
Client survey - ability with RP skills & $13.50(2.59)$ & 24 & $13.97(1.74)$ & 33 & .82
\end{tabular}

Note. $\mathrm{RP}=$ relapse prevention. 
assertiveness, anger, and relapse prevention, as well as confidence with various substance taking situations. Using Bonferroni's correction, a critical alpha of .004 was employed to control for the number of tests conducted. Overall, no significant differences were found between the two groups (see Table 11). Since the revised delivery format did not have an impact on postprogram outcomes, it can be suggested that offenders who participated in the original format were similar to the offenders who participated in the revised format.

\section{Missing Data on Noncompleters}

The sample included 34 offenders who did not complete the program, of which 14 (41.2\%) responded to postprogram questionnaires after departure from $\mathrm{MDH}$. The remaining 20 offenders (58.8\%) did not complete posttesting for a variety of reasons (see Table 12). The most prevalent source of incomplete data was due to offenders not mailing back the posttesting package to $\mathrm{MDH}$. Offenders refusing to complete posttesting after leaving the program accounted for $25.0 \%$ of missing posttests. In addition, $15.0 \%$ of noncompleters reached warrant expiry (i.e., the end of their sentence), so they could not be followed up for posttesting.

The two groups of noncompleters were compared across a number of demographic variables and pretest measures to determine whether a systematic pattern of missing data existed. Bonferroni's correction was used to control for the number of tests conducted using a critical alpha of .002 . Nonsignificant differences were found between the groups for age, education level, risk and need level, MDH's assessment of substance abuse treatment need, severity of substance abuse problem, and substance used most often, with this last item approaching significance $(\gamma=-1.00, p=.06)$. It should be noted 
Evaluation of a Correctional Substance Abuse Program 124

Table 11

Comparison Between Offenders who Did and Did Not Participate in the Revised Delivery of the Counselling and Cognitive-Behavioural Activities

\begin{tabular}{lccccc}
\hline & \multicolumn{5}{c}{ Group } \\
\cline { 2 - 5 } & $\begin{array}{c}\text { Original delivery } \\
\text { format }\end{array}$ & $\begin{array}{c}\text { Revised delivery } \\
\text { format }\end{array}$ & \\
Instrument & $M(S D)$ & $n$ & $M(S D)$ & $n$ & $t$ \\
\hline & & & & & \\
Completion/departure & & & & & \\
RCQ & $2.87(.34)$ & 23 & $3.00(.00)$ & 39 & -1.81 \\
PSQ & $58.96(15.34)$ & 24 & $58.92(13.20)$ & 37 & .01 \\
ASQ & $7.33(1.47)$ & 24 & $6.54(1.71)$ & 37 & -.91 \\
DTCQ negative situations & $92.20(12.01)$ & 23 & $94.16(12.42)$ & 38 & -.60 \\
DTCQ positive situations & $94.87(7.62)$ & 23 & $92.63(19.19)$ & 38 & .53 \\
DTCQ temptation situations & $86.55(23.84)$ & 23 & $89.65(18.67)$ & 38 & -.57 \\
Client survey - ability with problem solving & $4.32(.89)$ & 22 & $4.46(.70)$ & 35 & -.62 \\
Client survey - ability with assertiveness & $4.39(.72)$ & 23 & $4.37(.94)$ & 35 & .09 \\
Client survey - knowledge of anger & $4.57(.73)$ & 23 & $4.54(.66)$ & 35 & -.01 \\
Client survey - ability with anger & $4.39(.84)$ & 23 & $4.60(.61)$ & 35 & .98 \\
Client survey - knowledge of RP skills & $13.91(1.63)$ & 22 & $13.57(2.21)$ & 35 & .62 \\
Client survey - ability with RP skills & $13.77(2.11)$ & 22 & $13.77(2.17)$ & 35 & .002
\end{tabular}

Note. $\mathrm{RP}=$ relapse prevention. 
Table 12

Distribution of Reasons Why Noncompleters Did Not Have Posttesting $(n=20)$

Reason for no posttesting $\quad \%$

\begin{tabular}{lcc}
\hline Not mailed back to MDH & 35.0 & 7 \\
Refused to complete & 25.0 & 5 \\
Reached warrant expiry & 15.0 & 3 \\
UAL & 10.0 & 2 \\
Not accessible at OCDC & 10.0 & 2 \\
Returned to sender & 5.0 & 1 \\
\hline
\end{tabular}

Note. $\mathrm{MDH}=$ Maison Decision House Substance Abuse Treatment Program; UAL = unlawfully at large; OCDC $=$ Ottawa-Carleton Detention Center. 
that there were three cases among the noncompleters without posttesting who used alcohol most often, whereas the noncompleters with posttesting all used drugs most often.

Similarly, the groups did not differ on the following pretest measures: knowledge and ability with assertiveness and anger management skills, ability with relapse prevention skills, the BIDR IM and SDE subscales, RCQ, PSQ, ASQ, and the three subscales of the DTCQ. Three areas were identified in which the groups slightly diverged, although the tests only approached significance. Specifically, the offenders without posttesting had higher mean scores than the offenders with posttesting on knowledge of problem solving $(M=3.33, S D=.84 ; M=2.62, S D=.77$, respectively), $t(29)=2.43, p=.02$, and ability with problem solving $(M=3.50, S D=.92 ; M=2.46, S D$ $=.88$, respectively), $t(29)=3.15, p=.004$. Moreover, the offenders without posttesting had higher mean scores $(M=11.22, S D=2.92)$ than the offenders with posttesting $(M=$ $9.15, S D=2.23$ ) on knowledge of relapse prevention, $t(29)=2.14, p=.04$.

In general, no systematic differences were observed between the offenders without postprogram testing and offenders with postprogram testing on various demographic and pretest variables. Therefore, it can be concluded that the missing postprogram data for program noncompleters were random. Moreover, missing data were not likely to influence the results of the current evaluation.

Internal Consistency and Split-Half Estimates of the Client Survey, Program Staff Survey, and Pre- and Postvideo Questionnaires

The client and program staff surveys as well as the pre- and postvideo questionnaires were instruments developed for the current evaluation. Acceptable levels of internal consistency were found for the client survey and the program staff survey (see 
Appendix U, Table U1). Analysis revealed generally acceptable Cronbach's alphas for the four posttest video questionnaires ( $\alpha$ ranged from .59 to .69). However, low Cronbach's alphas were obtained with the pretest video questionnaires ( $\alpha$ ranged from .15 to .50 ). Principal components analysis was attempted to reduce the number of variables in the questionnaires, but this analysis could not be computed because at least one of the variables had zero variance. Thus, to examine the hypothesis related to the education component of the program, it was decided to combine the total scores for the anger, shame, denial, and addiction video questionnaires into a prevideo total score variable and a postvideo total score variable. The internal consistency estimates for the pre- and postvideo variables were acceptable (see Appendix U, Table U1). In general, the Guttman split-half demonstrated large correlations between the two parts of the client survey, staff survey, and video questionnaire (see Appendix U, Table U1).

Hypothesis 1-Program Completion Will Increase Knowledge of Prosocial Skills

Before beginning analysis of this hypothesis, possible covariates were examined. Results revealed that age and the preprogram BIDR SDE and IM subscale scores were not suitable covariates. Consequently, the analyses that follow do not incorporate any covariates. Risk level was also examined as a covariate and it was determined that risk level interacted with group; thus, MANCOVA was not suitable. Rather it was more appropriate to include risk level as an independent variable in the design (i.e., Attribute by Treatment Interaction design).

Offender self-report. A 2 (group: program completers and noncompleters) x 2 (test administration time: program intake and program completion/departure) $\mathrm{x} 3$ (risk level: low, medium, high) doubly multivariate repeated measures analysis of variance 
was conducted to assess the effects of program completion on five dependent variables associated with knowledge of prosocial skills (i.e., knowledge of assertiveness, anger, and problem-solving measured by the client survey, problem-solving measured by the PSQ, and assertiveness measured by the ASQ). The between-subjects effects were group and risk level, and the within-subjects effect was test administration time. Results are presented in Table 13.

The multivariate results for the between-subjects effect using Wilks' $\lambda$ demonstrated a significant difference between the groups on the set of dependent variables, $F(5,38)=6.00, p<.001, \eta_{p}^{2}=.44$. Univariate results showed that the difference between the two groups was significant for knowledge of problem-solving skills measured by the client survey. However, the two groups were not significantly different for knowledge of problem-solving skills measured by the PSQ. The univariate results were also nonsignificant for knowledge of assertiveness using the client survey and the ASQ. Although the effect was of medium size for knowledge of anger measured by the client survey, the finding was nonsignificant. For knowledge of anger, the power of the test was inadequate (.36), which likely contributed to the nonsignificant finding.

The multivariate results for the between-subjects effect using Wilks' $\lambda$ showed a nonsignificant difference between the three levels of risk on the set of the dependent variables, $F(10,76)=.81, p=.62, \eta_{\mathrm{p}}^{2}=.10$. In addition, a nonsignificant difference was found for the interaction between risk level and group, $F(10,76)=1.01, p=.38, \eta_{\mathrm{p}}^{2}=$ .13 .

A significant finding appeared for the within-subjects effect of test administration 
Table 13

Univariate Results from MANOVA Examining Knowledge of Prosocial Skills as a Function of Program Completion Based on Offender Self-Report

\begin{tabular}{|c|c|c|c|c|c|c|}
\hline Effect & & & $\bar{M}$ & $S E$ & $F$ & $\eta_{p}^{2}$ \\
\hline Between-su & & & & & & \\
\hline Group & & & & & & \\
\hline AssCs & PC & & 4.21 & .11 & 1.21 & .03 \\
\hline & $\mathrm{NC}$ & & 3.93 & .23 & & \\
\hline AngCS & $\mathrm{PC}$ & & 4.33 & .11 & 2.68 & .06 \\
\hline & $\mathrm{NC}$ & & 3.93 & .22 & & \\
\hline PrbCS & $\mathrm{PC}$ & & 4.26 & .11 & $23.66 * * *$ & .36 \\
\hline & $\mathrm{NC}$ & & 3.07 & .22 & & \\
\hline PSQ & $\mathrm{PC}$ & & 55.69 & 2.55 & .23 & .01 \\
\hline & $\mathrm{NC}$ & & 52.90 & 5.24 & & \\
\hline ASQ & $\mathrm{PC}$ & & 6.81 & .28 & .08 & .00 \\
\hline & $\mathrm{NC}$ & & 6.63 & .58 & & \\
\hline Within-subj & & & & & & \\
\hline Test time & & & & & & \\
\hline AssCS & Pre & & 3.81 & .20 & $6.29 *$ & .13 \\
\hline & Post & & 4.34 & .13 & & \\
\hline AngCS & Pre & & 3.93 & .18 & 3.56 & .08 \\
\hline & Post & & 4.34 & .15 & & \\
\hline PrbCS & Pre & & 3.19 & .20 & $19.12 * * *$ & .31 \\
\hline & Post & & 4.14 & .13 & & \\
\hline PSQ & Pre & & 50.65 & 3.74 & $4.71^{*}$ & .10 \\
\hline & Post & & 57.94 & 2.94 & & \\
\hline ASQ & Pre & & 6.94 & .38 & 1.71 & .04 \\
\hline & Post & & 6.95 & .35 & & \\
\hline Test time & & & & & & \\
\hline AssCS & Pre & $\mathrm{PC}$ & 3.82 & .17 & 1.56 & .04 \\
\hline & & $\mathrm{NC}$ & 3.80 & .35 & & \\
\hline & Post & $\mathrm{PC}$ & 4.61 & .11 & & \\
\hline & & $\mathrm{NC}$ & 4.07 & .23 & & \\
\hline
\end{tabular}


Evaluation of a Correctional Substance Abuse Program 130

\begin{tabular}{|c|c|c|c|c|c|c|}
\hline Effect & & & $M$ & $S E$ & $F$ & $\eta_{p}^{2}$ \\
\hline \multicolumn{7}{|c|}{ Test time $\mathrm{x}$ group } \\
\hline \multirow[t]{4}{*}{ AngCS } & Pre & $\mathrm{PC}$ & 3.99 & .16 & 1.61 & .04 \\
\hline & & $\mathrm{NC}$ & 3.87 & .32 & & \\
\hline & Post & PC & 4.67 & .13 & & \\
\hline & & $\mathrm{NC}$ & 4.00 & .26 & & \\
\hline \multirow[t]{4}{*}{ PrbCs } & Pre & $\mathrm{PC}$ & 3.84 & .17 & .27 & .01 \\
\hline & & $\mathrm{NC}$ & 2.53 & .35 & & \\
\hline & Post & $\mathrm{PC}$ & 4.68 & .11 & & \\
\hline & & $\mathrm{NC}$ & 3.60 & .23 & & \\
\hline \multirow[t]{4}{*}{ PSQ } & Pre & $\mathrm{PC}$ & 48.57 & 3.28 & $4.29 *$ & .10 \\
\hline & & $\mathrm{NC}$ & 52.73 & 6.72 & & \\
\hline & Post & PC & 62.81 & 2.58 & & \\
\hline & & $\mathrm{NC}$ & 53.07 & 5.28 & & \\
\hline \multirow[t]{4}{*}{ ASQ } & Pre & $\mathrm{PC}$ & 6.58 & .33 & .00 & .00 \\
\hline & & $\mathrm{NC}$ & 6.40 & .68 & & \\
\hline & Post & $\mathrm{PC}$ & 7.04 & .31 & & \\
\hline & & $\mathrm{NC}$ & 6.87 & .64 & & \\
\hline \multicolumn{7}{|c|}{ Test time $\mathrm{x}$ risk } \\
\hline \multirow[t]{6}{*}{ AssCS } & Pre & Low & 3.80 & .49 & .59 & .03 \\
\hline & & Medium & 3.53 & .22 & & \\
\hline & & High & 4.10 & .24 & & \\
\hline & Post & Low & 4.20 & .32 & & \\
\hline & & Medium & 4.30 & .15 & & \\
\hline & & High & 4.52 & .16 & & \\
\hline \multirow[t]{6}{*}{ AngCS } & Pre & Low & 4.00 & .44 & .56 & .03 \\
\hline & & Medium & 3.88 & .20 & & \\
\hline & & High & 3.92 & .22 & & \\
\hline & Post & Low & 4.30 & .37 & & \\
\hline & & Medium & 4.15 & .17 & & \\
\hline & & High & 4.56 & .18 & & \\
\hline
\end{tabular}


Evaluation of a Correctional Substance Abuse Program 131

\begin{tabular}{|c|c|c|c|c|c|c|}
\hline Effect & & & $\bar{M}$ & $S E$ & $F$ & $\overline{\eta_{p}^{2}}$ \\
\hline \multicolumn{7}{|c|}{ Test time $\mathrm{x}$ risk } \\
\hline \multirow[t]{6}{*}{ PrbCS } & Pre & Low & 3.00 & .49 & .12 & .01 \\
\hline & & Medium & 3.13 & .22 & & \\
\hline & & High & 3.43 & .24 & & \\
\hline & Post & Low & 3.80 & .31 & & \\
\hline & & Medium & 4.10 & .14 & & \\
\hline & & High & 4.52 & .15 & & \\
\hline \multirow[t]{6}{*}{ PSQ } & Pre & Low & 59.70 & 9.33 & $5.08^{*}$ & .20 \\
\hline & & Medium & 48.95 & 4.26 & & \\
\hline & & High & 43.30 & 4.53 & & \\
\hline & Post & Low & 60.90 & 7.33 & & \\
\hline & & Medium & 50.80 & 3.35 & & \\
\hline & & High & 62.12 & 3.56 & & \\
\hline \multirow[t]{6}{*}{ ASQ } & Pre & Low & 6.10 & .94 & 1.05 & .05 \\
\hline & & Medium & 6.86 & .43 & & \\
\hline & & High & 6.49 & .46 & & \\
\hline & Post & Low & 7.20 & .88 & & \\
\hline & & Medium & 6.73 & .40 & & \\
\hline & & High & 6.93 & .43 & & \\
\hline
\end{tabular}

Note. Group $=$ program completers $(\mathrm{PC})$ and noncompleters $(\mathrm{NC})$; Test time $=$ preprogram $(\mathrm{Pre})$ and postprogram (Post); Risk $=$ low, medium and high risk levels; AssCS $=$ knowledge of assertiveness measured by the client survey; AngCS = knowledge of anger measured by the client survey; PrbCS = knowledge of problem solving measured by the client survey; PSQ = knowledge of problem solving measured by the Problem-Solving Questionnaire; ASQ = knowledge of assertiveness measured by the Assertiveness Questionnaire. For the program completer group $n=37$ and $n=11$ for the noncompleter group.

${ }^{*} p<.05 .{ }^{* * *} p<.001$ 
time on the set of dependent variables, $F(5,38)=4.36, p<.01, \eta_{\mathrm{p}}^{2}=.37$. Univariate results revealed significant differences from pre- to posttest for knowledge of assertiveness and knowledge of problem solving measured by the client survey and the PSQ (see Table 13). Knowledge of assertiveness measured by the ASQ did not differ significantly from pretest to posttest. Knowledge of anger measured by the client survey had a medium to large effect size, but was not significant. It is likely that this test would have reached significance if the power of the test was higher (power $=.45$ ).

The test administration time by group interaction was not significant on the set of dependent variables, $F(5,38)=1.83, p=.13, \eta_{\mathrm{p}}^{2}=.19$ ). It is possible that the nonsignificant result was due to the low power of the test (.56). Nonetheless, since the interaction was a hypothesis and the nonsignificant result had a medium-large effect size, the univariate results were examined. The univariate findings indicated that knowledge of problem solving measured by the PSQ was significant, that is, program completers had more knowledge of problem-solving skills at the end of the program than noncompleters did at program departure (see Table 13). All other results were nonsignificant. However, an examination of the profile plots revealed trends in the expected direction for knowledge of assertiveness and anger measured by the client survey, with effect sizes in the small-medium range (see Appendix V, Figures V1 and V2). Specifically, it appears that there is a larger difference between the two groups at posttest than pretest, with completers having a higher mean score than noncompleters for reported increases in knowledge of assertiveness and anger.

The test administration time by risk interaction was significant on the set of dependent variables, $F(10,76)=2.29, p<.05, \eta_{\mathrm{p}}^{2}=.23$. The simple effects of test 
administration time at each level of risk were examined. Multivariate results showed that there were significant differences between pre- and posttesting for medium risk offenders, $F(5,38)=4.67, p<.01, \eta_{\mathrm{p}}^{2}=.38$, and high risk offenders, $F(5,38)=6.53, p<$ $.001, \eta_{p}^{2}=.46$. However, no significant difference was found due to test administration time for low risk offenders, $F(5,38)=.91, p=.49, \eta_{\mathrm{p}}^{2}=.10$.

An examination of the univariate results indicated that medium risk offenders had significantly higher mean scores for knowledge of assertiveness and problem-solving measured by the client survey at posttest. Moreover, high risk offenders had significantly higher mean scores for knowledge of anger on the client survey and for knowledge of problem-solving on both the client survey and the PSQ at posttest (see Table 14).

Program staff assessment. Prosocial skills knowledge was also examined using the staff survey on offender knowledge of assertiveness, anger, and problem-solving skills at the time the offender finished or left the program. A MANOVA was conducted to determine whether program completers differed from noncompleters on the set of prosocial skills. Using Wilks' $\lambda$, the multivariate results showed that the groups differed significantly on the linear combination of the dependent variables, $F(3,74)=9.51, p<$ $.001, \eta_{\mathrm{p}}^{2}=.28$.

The univariate results demonstrated significant differences between the groups on the three dimensions of prosocial skills, with program completers obtaining higher scores than the noncompleters (see Table 15). Examination of the effect sizes revealed that counsellor feedback on problem-solving skills knowledge $\left(\eta_{p}^{2}=.26\right)$, anger knowledge $\left(\eta_{\mathrm{p}}^{2}=.20\right)$, and assertiveness knowledge $\left(\eta_{\mathrm{p}}^{2}=.19\right)$ were good discriminators of program completers from noncompleters, with large effect sizes. 
Evaluation of a Correctional Substance Abuse Program 134

Table 14

Univariate Results for Simple Effects of Test Administration Time at Each Level of Risk

\begin{tabular}{|c|c|c|c|c|}
\hline \multirow[b]{4}{*}{ Variable } & \multicolumn{2}{|c|}{ Test administration time } & \multirow[b]{3}{*}{ Mean } & \multirow[b]{4}{*}{$p$} \\
\hline & Pre & Post & & \\
\hline & \multirow[b]{2}{*}{$M(S E)$} & & & \\
\hline & & $M(S E)$ & difference & \\
\hline \multicolumn{5}{|l|}{ AssCS } \\
\hline Low & $3.80(.49)$ & $4.20(.32)$ & -.40 & .45 \\
\hline Medium & $3.53(.22)$ & $4.30(.15)$ & $-.78^{* *}$ & .003 \\
\hline High & $4.10(.24)$ & $4.52(.16)$ & .42 & .11 \\
\hline \multicolumn{5}{|l|}{ AngCS } \\
\hline Low & $4.00(.44)$ & $4.30(.37)$ & -.30 & .58 \\
\hline Medium & $3.88(.20)$ & $4.15(.17)$ & -.28 & .27 \\
\hline High & $3.92(.22)$ & $4.56(.18)$ & $-.64^{*}$ & .02 \\
\hline \multicolumn{5}{|l|}{ Prbcs } \\
\hline Low & $3.00(.49)$ & $3.80(.31)$ & -.80 & .15 \\
\hline Medium & $3.13(.22)$ & $4.10(.14)$ & $-.98 * * *$ & .00 \\
\hline High & $3.43(.24)$ & $4.52(.15)$ & $-1.08 * * *$ & .00 \\
\hline \multicolumn{5}{|l|}{ PSQ } \\
\hline Low & $59.70(9.33)$ & $60.90(7.33)$ & -1.20 & .89 \\
\hline Medium & $48.95(4.26)$ & $50.80(3.35)$ & -1.85 & .63 \\
\hline High & $43.30(4.53)$ & $62.12(3.56)$ & $-18.82 * * *$ & .00 \\
\hline \multicolumn{5}{|l|}{ ASQ } \\
\hline Low & $6.10(.94)$ & $7.20(.88)$ & -1.10 & .22 \\
\hline Medium & $6.88(.43)$ & $6.73(.40)$ & .15 & .71 \\
\hline High & $6.49(.46)$ & $6.93(.43)$ & -.44 & .31 \\
\hline \multicolumn{5}{|c|}{$\begin{array}{l}\text { Note. AssCS = knowledge of assertiveness measured by the client survey; AngCS = knowledge of anger } \\
\text { measured by the client survey; PrbCS = knowledge of problem solving measured by the client survey; PSQ } \\
=\text { knowledge of problem solving measured by the Problem-Solving Questionnaire; ASQ = knowledge of } \\
\text { assertiveness measured by the Assertiveness Questionnaire; Low = low risk; Medium = medium risk; High } \\
\text { = high risk. }\end{array}$} \\
\hline \multicolumn{5}{|c|}{${ }^{*} p<.05 . * * p<.01 . * * * p<.001$} \\
\hline
\end{tabular}


Evaluation of a Correctional Substance Abuse Program 135

Table 15

Program Staff Assessment on Knowledge of Prosocial Skills by Group Postprogram

\begin{tabular}{lcccc}
\hline & \multicolumn{3}{c}{ Group } & \\
\cline { 2 - 3 } Prosocial skill & $M(S D)$ & $M(S D)$ & $F$ & $\eta^{2} \mathrm{p}$ \\
\hline Assertiveness & $4.29(.80)$ & $3.47(.94)$ & $17.24^{* * *}$ & .19 \\
Anger & $4.25(.73)$ & $3.40(.97)$ & $19.42^{* * * *}$ & .20 \\
Problem-solving & $4.23(.81)$ & $3.20(.93)$ & $26.88^{* * *}$ & .26 \\
\hline
\end{tabular}

Note. For the program completer group $n=48$ and $n=30$ for the noncompleter group.

$* * * p<.001$. 
In summary, the program completers and noncompleters differ on their knowledge of problem-solving skills, with completers having higher mean scores than the noncompleters. Increases in knowledge were found on assertiveness and problem solving from pretest to posttest. Medium risk offenders had more knowledge on assertiveness and problem solving at posttest than pretest. In addition, high risk offenders demonstrated the same pattern of findings on knowledge of anger and problem-solving skills across test administration time.

Significant results were not discovered for the interaction between group and test administration time as hypothesized. This finding suggests that there was no relationship between program completion and increased knowledge of prosocial skills; however, the profile plots show trends for knowledge of assertiveness, anger, and problem solving in the expected direction, which supports the original hypothesis. In support of the trends found using offender self-reports, significant differences were obtained using staff ratings. Specifically, program staff reported that offenders who completed the program had more knowledge on assertiveness, anger, and problem solving than offenders who did not complete the program.

\section{Hypothesis 2 - Program Completion Will Increase Knowledge of Relapse Prevention} Skills

An examination of the relationships among the dependent variables revealed large correlations among the three items measuring knowledge of relapse prevention skills on the pre- and postprogram client survey and the postprogram staff survey. Correlations between pairs of dependent variables ranged from $r=.67$ to $r=.91$ (all $p \mathrm{~s}<.001$ ). Given the high intercorrelations among the items, three principal components analyses were 
conducted using a varimax rotation to determine whether the three items on each survey contributed to a single construct. The results for the pre- and postprogram client survey and the staff ratings showed that the three items loaded on one factor (see Appendix U, Table U2). This new subscale was named knowledge of relapse prevention skills. Thus, it was decided that analyses of hypothesis 2 would be conducted using a sum of the three items on the pre- and postprogram client surveys and on the postprogram staff survey. The internal consistency estimates for the knowledge of relapse prevention skills subscales were acceptable: pretest on client survey, $\alpha=.88$; posttest on client survey, $\alpha=$ .94 ; and posttest on staff survey, $\alpha=.93$.

Offender self-report. To examine the effects of program completion on knowledge of relapse prevention skills a mixed ANOVA was conducted. The withinsubjects effect was test administration time and the between-subjects effect was group. The result for the within-subjects effect showed a significant difference in knowledge over time, $F(1,46)=11.42, p<.001, \eta_{\mathrm{p}}^{2}=.20$. For the between-subjects effect, a significant difference was found in knowledge levels between program completers and noncompleters, $F(1,46)=17.61, p<.001, \eta_{p}^{2}=.28$.

The interaction between test administration time and group was not significant, $F(1,46)=2.34, p=.13, \eta_{\mathrm{p}}^{2}=.05$. Since this interaction was a hypothesis and the effect size was small-medium, the profile plot was examined revealing the expected trend (see Appendix V, Figure V3). Specifically, it appears that there is a larger difference between the two groups at posttest than pretest, with completers having a higher mean score than noncompleters for reported increases in knowledge of relapse prevention skills (see Table 16). 
Evaluation of a Correctional Substance Abuse Program 138

Table 16

Offender Self-Report on Knowledge of Relapse Prevention Skills by Group

\begin{tabular}{|c|c|c|c|c|c|c|}
\hline \multirow[b]{4}{*}{ Knowledge } & \multicolumn{4}{|c|}{ Group } & \multirow[b]{4}{*}{$F$} & \multirow[b]{4}{*}{$\eta_{p}^{2}$} \\
\hline & \multicolumn{2}{|c|}{ Program completers $(n=38)$} & \multicolumn{2}{|c|}{ Noncompleters $(n=10)$} & & \\
\hline & Pre & Post & Pre & Post & & \\
\hline & $M(S D)$ & $M(S D)$ & $M(S D)$ & $M(S D)$ & & \\
\hline $\begin{array}{l}\text { Relapse } \\
\text { prevention } \\
\text { skills }\end{array}$ & $11.24(3.05)$ & $14.16(1.28)$ & $9.70(2.21)$ & $10.80(2.49)$ & 2.34 & .05 \\
\hline
\end{tabular}


Program staff assessment. An ANCOVA was conducted using age as a covariate to examine knowledge of relapse prevention skills as a result of program completion. Results showed a significant effect for group, $F(1,75)=23.21, p<.001, \eta_{\mathrm{p}}^{2}=.24$. Specifically, staff rated offenders who completed the program as having more knowledge of relapse prevention skills $(M=13.02, S D=2.00)$ than offenders who did not complete the program $(M=9.93, S D=2.88)$.

In summary, knowledge of relapse prevention skills measured by the client survey failed to demonstrate a significant interaction with program completion as hypothesized. Although this finding suggests that there was no relationship between program completion and increased knowledge of relapse preventions skills, the profile plot illustrates the expected trend, which is consistent with the original hypothesis. In support of the trend found using the offender self-report, a significant difference was obtained using staff ratings, that is, program staff reported that offenders who completed the program had more knowledge of relapse prevention skills than offenders who did not finish the program.

Hypothesis 3 - Program Completion Will Increase Ability With Prosocial Skills

Offender self-report. A 2 (group: program completers and noncompleters) x 2 (test administration time: program intake and program completion/departure) doubly multivariate repeated measures analysis of covariance was conducted to assess the effects of program completion on the three dependent variables associated with ability on prosocial skills (i.e., ability with assertiveness, anger, and problem-solving measured by the client survey). The between-subjects effect was group and the within-subjects effect was test administration time. Age was used as a covariate. 
The multivariate results for the between-subjects effect using Wilks' $\lambda$ showed a significant difference between the groups on the set of dependent variables, $F(3,43)=$ $8.83, p<.001, \eta_{p}^{2}=.38$. Univariate results demonstrated that the difference between the two groups was significant for ability with anger and problem solving (see Table 17). Although the effect was of medium size for ability with assertiveness, the finding was nonsignificant. This nonsignificant result is most likely due the low power of the test $(.36)$.

For the within-subjects effect, the multivariate results revealed test administration time was not significant on the set of dependent variables, $F(3,43)=2.12, p=.11, \eta_{p}^{2}=$ .13. Although nonsignificant, the magnitude of this result was moderate. The interaction between group and test administration time was significant on the set of dependent variables, $F(3,43)=3.11, p<.05, \eta_{\mathrm{p}}^{2}=.18$, which supports the original hypothesis. Even though the univariate findings showed that ability with assertiveness only approached significance $(p=.06)$, a medium effect size was found, which indicates that the groups do indeed differ. The profile plot of this interaction illustrates the expected pattern of results (see Appendix V, Figure V4). Specifically, program completers had more ability with assertiveness after finishing the program than offenders who did not complete the program. Results showed nonsignificant findings for ability with anger and problem solving.

Program staff assessment. Ability with prosocial skills was also examined using the staff survey on offender ability with assertiveness, anger management, and problem solving at the time the offender finished or left the program. A MANCOVA was 
Evaluation of a Correctional Substance Abuse Program 141

Table 17

Univariate Results from MANCOVA Examining Ability with Prosocial Skills as a

Function of Program Completion Based on Offender Self-Report

\begin{tabular}{lcccc}
\hline \multirow{2}{*}{ Effect } & Unadjusted & Adjusted & & \\
& $M(S E)$ & $M(S E)$ & $F$ & $\eta_{\mathrm{p}}^{2}$ \\
\hline
\end{tabular}

Between-subjects effects

Group

AssCS

PC

$4.00(.10)$

$4.20(.11)$

$2.63 \quad .06$

AngCS

$\mathrm{NC}$

$3.42(.21)$

$3.80(.29)$

PC

$4.16(.10)$

$4.38(.10)$

$5.28 * \quad .11$

PrbCS

$\mathrm{NC}$

$3.54(.20)$

$3.91(.18)$

$4.07(.11)$

$4.21(.10)$

$25.07 * * * \quad .36$

$\mathrm{NC}$

$2.88(.23)$

$3.05(.20)$

Within-subjects effects

Test time

$\begin{array}{lllllll}\text { AssCS } & \text { Pre } & & 3.86(.11) & 3.76(.17) & 1.83 & .04 \\ & \text { Post } & & 4.38(.11) & 4.23(.11) & & \\ \text { AngCS } & \text { Pre } & & 4.06(.11) & 4.00(.14) & .03 & .08 \\ & \text { Post } & & 4.52(.09) & 4.28(.12) & & \\ \text { PrbCS } & \text { Pre } & & 3.51(.13) & 3.16(.17) & 2.23 & .05 \\ & \text { Post } & & 4.40(.10) & 4.10(.11) & & \\ \text { Test time x group } & & & & & & \\ \text { AssCS } & \text { Pre } & \text { PC } & 3.84(.15) & 3.82(.15) & 3.69^{\dagger} & .08 \\ & & \text { NC } & 3.60(.27) & 3.71(.27) & & \\ & \text { Post } & \text { PC } & 4.61(.10) & 4.58(.10) & & \\ & & \text { NC } & 3.80(.25) & 3.88(25) & & \end{array}$




\begin{tabular}{lcccc}
\hline & Unadjusted & Adjusted & & \\
Effect & $M(S E)$ & $M(S E)$ & $F$ & $\eta_{\mathrm{p}}^{2}$ \\
\hline
\end{tabular}

Test time $\mathrm{x}$ group

$\begin{array}{lllllll}\text { AngCS } & \text { Pre } & \text { PC } & 4.18(.12) & 4.18(.12) & .49 & .01 \\ & & \text { NC } & 3.80(.25) & 3.83(.25) & & \\ \text { PrbCS } & \text { PC } & 4.61(.11) & 4.58(.11) & & \\ & & \text { NC } & 3.90(.23) & 3.98(.23) & & \\ & \text { Pre } & \text { PC } & 3.84(.15) & 3.82(.15) & .75 & .02 \\ & & \text { NC } & 2.40(.27) & 2.50(.27) & & \\ & \text { Post } & \text { PC } & 4.63(.10) & 4.60(.10) & & \\ & & \text { NC } & 3.50(.22) & 3.61(.22) & \end{array}$

Note. Adjusted means evaluated at mean of covariate age $=35.92$. Group $=$ program completers $(\mathrm{PC})$ and noncompleters $(\mathrm{NC})$; Test time = preprogram (Pre) and postprogram (Post); AssCS = knowledge of assertiveness measured by the client survey; AngCS = knowledge of anger measured by the client survey; PrbCS = knowledge of problem solving measured by the client survey. For the program completer group $n=38$ and $n=$ 10 for the noncompleter group.

${ }^{\dagger} p<.10 .{ }^{*} p<.05 . * * * p<.001$ 
conducted, with age and risk level as covariates, to determine whether program completers differed from noncompleters on the set of prosocial skills with respect to ability. The multivariate results using Wilks' $\lambda$ showed a significant effect for group, $F(3$, $72)=4.67, p<.01, \eta_{\mathrm{p}}^{2}=.16$. Univariate results are presented in Table 18. The findings revealed that staff rated offenders who completed the program as having more ability with anger and problem solving than offenders who did not complete the program. No significant group difference was found for ability with assertiveness, but the mean difference was in the expected direction.

In summary, the program completers and noncompleters differed on their ability for anger and problem-solving skills, with completers having higher mean scores than the noncompleters. No significant differences were observed for increases in ability with assertiveness from pretest to posttest, although the effect was of medium magnitude. A significant interaction was detected for group by test administration time as hypothesized. However, the only prosocial skill to approach significance with a medium effect size was ability with assertiveness. The staff ratings supported part of the original hypothesis. Specifically, staff reported that offenders who completed the program had more ability with anger and problem solving than offenders who did not complete the program.

\section{Hypothesis 4 - Program Completion Will Increase Ability with Relapse Prevention Skills}

An examination of the relationships among the dependent variables revealed large correlations among the three items on the pre- and postprogram client survey and the postprogram staff survey, which measure ability of relapse prevention skills. Correlations between pairs of dependent variables ranged from $r=.66$ to $r=.82$ (all $p \mathrm{~s}<.001$ ). Given the high intercorrelations among the items, three principal components analyses were 
Table 18

Univariate Results from MANCOVA Examining Ability with Prosocial Skills as a

Function of Program Completion Based on Program Staff Assessment

\begin{tabular}{lcccc}
\hline & Unadjusted & Adjusted & & \\
Effect & $M(S E)$ & $M(S E)$ & $F$ & $\eta_{\mathrm{p}}^{2}$ \\
\hline
\end{tabular}

Between-subjects effects

Group

$\begin{array}{llllll}\text { AssSS } & \text { PC } & 3.75(.14) & 3.64(.15) & 2.60 & .03 \\ & \text { NC } & 3.07(.21) & 3.24(.19) & & \\ \text { AngSS } & \text { PC } & 3.88(.12) & 3.79(.14) & 12.81^{* *} & .15 \\ & \text { NC } & 2.80(.22) & 2.95(.18) & & \\ \text { PrbSS } & \text { PC } & 3.65(.13) & 3.54(.13) & 10.41^{* *} & .12 \\ & \text { NC } & 2.67(.17) & 2.84(.16) & & \end{array}$

Note. Adjusted means evaluated at mean of covariate age $=35.10$. Adjusted means

evaluated at mean of covariate risk level $=2.32$. Group $=$ program completers $(P C)$ and noncompleters $(\mathrm{NC})$; AssSS = knowledge of assertiveness measured by the staff survey; AngSS = knowledge of anger measured by the staff survey; PrbSS = knowledge of problem solving measured by the staff survey. For the program completer group $n=48$ and $n=30$ for the noncompleter group.

$* * p<.01$. 
conducted using a varimax rotation to determine whether the three items on each survey contributed to a single construct. The results for the pre- and postprogram client survey and the staff ratings showed that the three items loaded on one factor (see Appendix $U$, Table U3). This new subscale was named ability with relapse prevention skills. Thus, it was decided that analyses of hypothesis 4 would be conducted using a sum of the three items on the pre- and postprogram client surveys and on the postprogram staff survey. The internal consistency estimates for the ability of relapse prevention skills subscales were acceptable: pretest on client survey, $\alpha=.90$; posttest on client survey, $\alpha=.91$; and posttest on staff survey, $\alpha=.89$.

Offender self-report. To examine the effects of program completion on ability with relapse prevention skills, a mixed ANCOVA was run with the preprogram BIDR SDE subscale as a covariate. The between-subjects effect was group and the withinsubjects effect was test administration time. The result for the between-subjects effect demonstrated a significant difference between the groups for ability levels, $F(1,45)=$ $15.56, p<.001, \eta_{\mathrm{p}}^{2}=.26$. For the within-subjects effect, a significant difference was found in ability over time, $F(1,45)=12.30, p<.01, \eta^{2}=.22$.

The interaction between test administration time and group was also significant, $F(1,45)=8.38, p<.01, \eta_{\mathrm{p}}^{2}=.16$. This analysis showed that there was a larger difference between the two groups at posttest than pretest, with completers having a higher mean score than noncompleters for reported increases in ability with relapse prevention skills (see Table 19).

Program staff assessment. An ANCOVA was conducted to examine ability with relapse prevention skills as a result of program completion using age and the preprogram 
Evaluation of a Correctional Substance Abuse Program 146

Table 19

Univariate Results from ANCOVA Examining Ability with Relapse Prevention Skills as a Function of Program Completion Based on Offender Self-Report

\begin{tabular}{lccccc}
\hline & Unadjusted & Adjusted & & \\
Effect & $M(S E)$ & $M(S E)$ & $F$ & $\eta^{2} \mathrm{p}$ \\
\hline
\end{tabular}

Within-subjects effects

Test time $\mathrm{x}$ group $8.38^{* *} .16$ RP-CS PC Pre $11.11(.50)$ $10.92(.46)$

$\begin{array}{rlcc} & \text { Post } & 14.45(.35) & 14.43(.25) \\ \text { NC } & \text { Pre } & 9.70(.34) & 10.39(.92) \\ & \text { Post } & 10.30(.80) & 10.38(.50)\end{array}$

Note. Adjusted means evaluated at mean of covariate preprogram BIDR SDE subscale $=$ 6.62. Group $=$ program completers $(\mathrm{PC})$ and noncompleters $(\mathrm{NC})$; Test time $=$ preprogram (Pre) and postprogram (Post); RP-CS = ability with relapse prevention skills measured by the client survey. For the program completer group $n=38$ and $n=10$ for the noncompleter group.

$* * p<.01$. 
BIDR IM subscale as covariates. Results revealed a significant effect for group, $F(1,73)$ $=18.60, p<.001, \eta_{p}^{2}=.20$. Specifically, staff rated offenders who completed the program as having more ability with relapse prevention skills (adjusted $M=11.43, S E=$ .39 , unadjusted $M=11.60, S E=.55)$ than offenders who did not complete the program (adjusted $M=8.57, S D=.51$, unadjusted $M=8.28, S D=.34$ ).

Therefore, as hypothesized, ability with relapse prevention skills increased as a function of program completion. Staff ratings supported this finding. Program staff reported that offenders who completed the program had more ability with relapse prevention skills than offenders who did not finish the program.

\section{Hypothesis 5 - Program Completion Will Increase Confidence to Not Use Substances}

Offender self-report. Examinations of the pretest and posttest subscales of the DTCQ demonstrated high correlations among the variables at each test administration time. Correlations between the pretest measures ranged from .60 to .91 (see Table 20). The posttest measures demonstrated the same pattern, with correlations ranging from .54 to .94 (see Appendix U, Table U4). To examine multicollinearity, eight multiple regression analyses were run predicting each dependent variable from all other dependent variables. A variance inflation factor (VIF) greater than 10 and a tolerance less than .10 are indicative of multicollinearity (Stevens, 2002). Four of the regression analyses produced a VIF close to $10(9.38$ to 9.65$)$ and tolerances of .10 . Therefore, it was decided to combine the eight subscales into three pre-existing subscales that represent a threefactor model consisting of: (1) negative situations, (2) positive situations, and (3) temptation situations. This three-factor model is supported by empirical evidence and has clinical utility (Annis et al., 1997). Multicollinearity was examined for the new three- 
Evaluation of a Correctional Substance Abuse Program 148

Table 20

Correlation Matrix for the Pretest Measures of the DTCQ $(n=81)$

\begin{tabular}{|c|c|c|c|c|c|c|c|c|}
\hline & DTCQ 1 & DTCQ 2 & DTCQ 3 & DTCQ 4 & DTCQ 5 & DTCQ 6 & DTCQ 7 & DTCQ 8 \\
\hline DTCQ 1 & - & & & & & & & \\
\hline DTCQ 2 & .76 & - & & & & & & \\
\hline DTCQ 3 & .82 & .87 & - & & & & & \\
\hline DTCQ 4 & .73 & .75 & .81 & - & & & & \\
\hline DTCQ 5 & .74 & .78 & .82 & .71 & - & & & \\
\hline DTCQ 6 & .75 & .74 & .76 & .81 & .70 & - & & \\
\hline DTCQ 7 & .70 & .67 & .73 & .84 & .68 & .91 & -- & \\
\hline DTCQ 8 & .63 & .60 & .64 & .73 & .60 & .86 & .88 & -- \\
\hline
\end{tabular}

Note. All correlations were significant at $p<.001$. 
factor model, and it was determined that all of the VIFs were less than 10 (i.e., 3.3, 2.4, and 4.8).

A 2 (group: program completers and noncompleters) x 2 (test administration time: program intake and program completion/departure) doubly multivariate repeated measures analysis of covariance was conducted to assess the effects of program completion on four dependent variables associated with confidence (i.e., overall confidence in avoiding substances measured by the client survey and confidence to not use substances in negative, positive, and temptation situations measured by the DTCQ). The covariates incorporated into the analysis were the preprogram BIDR SDE subscale and the preprogram BIDR IM subscale. The between-subjects effect was group and the within-subjects effect was test administration time.

Box's test assesses the homogeneity of covariance matrices assumption in MANOVA. Since Box's $M$ was significant, $F(36,893)=5.02, p<.001$, the generalized variances were examined to determine whether the multivariate test would be robust to the violation. If the largest generalized variance is associated with the smallest group size, the multivariate test statistic will be too liberal. To correct for a liberal test, it is recommended that a more stringent alpha of .01 be used to interpret the multivariate statistics (Stevens, 2002). Since this was the case in the current analysis, a critical alpha of .01 was employed.

The multivariate results for the between-subjects effect using Wilks' $\lambda$ showed a nonsignificant difference between the groups on the set of dependent variables, $F(4,48)$ $=2.63, p=.05, \eta_{\mathrm{p}}^{2}=.18$. Multivariate results for the within-subjects effect of test administration time on the set of dependent variables approached significance, $F(4,48)=$ 
$2.22, p=.08, \eta_{\mathrm{p}}^{2}=.16$, with a medium effect size. The test administration time by group interaction was significant on the set of dependent variables, $F(4,48)=3.99, p<.01, \eta^{2}$ p $=.25$, supporting the original hypothesis.

The univariate findings indicated that the interactions were significant for overall confidence to avoid substances measured by the client survey and for confidence to not use substances in negative situations measured by the DTCQ. Specifically, program completers had more confidence to avoid substances and confidence to not use substances in negative situations at the end of program than noncompleters did at program departure (see Table 21). The test administration by group interaction approached significance for confidence to not take substances in temptation situations, with a medium effect size. A nonsignificant univariate result was found for confidence to not use substances in positive situations. An examination of the profile plots for these two nonsignificant findings illustrates a pattern of effects consistent with the hypothesis (see Appendix V, Figures V5 and V6).

In support of the original hypothesis, results showed that program completers had more overall confidence in substance avoidance and confidence to not use substances in negative situations than noncompleters. Further evidence of this interaction was evident in the profile plots for confidence to not take substances in positive and temptation situations, with program completers appearing to have more confidence in both types of situations at the end of the program in comparison to noncompleters. 
Evaluation of a Correctional Substance Abuse Program 151

Table 21

Univariate Results from MANCOVA Examining Increases in Confidence as a Function of Program Completion Based on Offender Self-Report

\begin{tabular}{|c|c|c|c|c|c|c|}
\hline Effect & & & $\begin{array}{c}\text { Unadjusted } \\
M(S E)\end{array}$ & $\begin{array}{c}\text { Adjusted } \\
M(S E)\end{array}$ & $F$ & $\eta_{p}^{2}$ \\
\hline \multicolumn{7}{|c|}{ Between-subjects effects } \\
\hline \multicolumn{7}{|l|}{ Group } \\
\hline \multirow[t]{2}{*}{ ConfCS } & $\mathrm{PC}$ & & $4.78(.09)$ & $4.76(.09)$ & $8.08^{* * *}$ & .14 \\
\hline & $\mathrm{NC}$ & & $4.10(.18)$ & $4.16(.19)$ & & \\
\hline \multirow[t]{2}{*}{ NegsitDTCQ } & $\mathrm{PC}$ & & $88.72(1.91)$ & $88.15(1.86)$ & 1.70 & .03 \\
\hline & $\mathrm{NC}$ & & $79.70(4.05)$ & $82.27(4.04)$ & & \\
\hline \multirow[t]{2}{*}{ PossitDTCQ } & $\mathrm{PC}$ & & $89.29(1.96)$ & $88.84(1.95)$ & $4.26^{*}$ & .08 \\
\hline & $\mathrm{NC}$ & & $77.00(4.16)$ & $79.07(4.25)$ & & \\
\hline \multirow[t]{2}{*}{ TempsitDTCQ } & $\mathrm{PC}$ & & $84.00(2.94)$ & $83.17(2.88)$ & 3.82 & .07 \\
\hline & $\mathrm{NC}$ & & $65.77(6.24)$ & $69.53(6.27)$ & & \\
\hline \multicolumn{7}{|c|}{ Within-subjects effects } \\
\hline \multicolumn{7}{|l|}{ Test time } \\
\hline \multirow[t]{2}{*}{ ConfCS } & Pre & & $4.44(.15)$ & $4.48(.15)$ & 1.60 & .03 \\
\hline & Post & & $4.44(.09)$ & $4.44(.09)$ & & \\
\hline \multirow[t]{2}{*}{ NegsitDTCQ } & Pre & & $79.57(4.23)$ & $81.34(4.18)$ & $7.40^{* *}$ & .13 \\
\hline & Post & & $88.84(1.32)$ & $89.07(1.35)$ & & \\
\hline \multirow[t]{2}{*}{ PossitDTCQ } & Pre & & $76.85(4.30)$ & $78.43(4.30)$ & $8.82 * *$ & .15 \\
\hline & Post & & $89.44(1.61)$ & $89.47(1.66)$ & & \\
\hline \multirow[t]{2}{*}{ TempsitDTCQ } & Pre & & $69.06(5.39)$ & $71.14(5.36)$ & $5.03^{*}$ & .09 \\
\hline & Post & & $80.71(2.81)$ & $81.56(2.85)$ & & \\
\hline \multicolumn{7}{|l|}{ Test time $\mathrm{x}$ group } \\
\hline \multirow[t]{4}{*}{ ConfCS } & Pre & $\mathrm{PC}$ & $4.58(.13)$ & $4.55(.13)$ & $9.72 * *$ & .16 \\
\hline & & $\mathrm{NC}$ & $4.30(.27)$ & $4.42(.28)$ & & \\
\hline & Post & PC & $4.98(.08)$ & $4.98(.08)$ & & \\
\hline & & $\mathrm{NC}$ & $3.90(.16)$ & $3.91(.17)$ & & \\
\hline \multirow[t]{4}{*}{ NegsitDTCQ } & Pre & $\mathrm{PC}$ & $80.14(3.60)$ & $79.13(3.53)$ & $5.25^{*}$ & .09 \\
\hline & & $\mathrm{NC}$ & $79.00(7.65)$ & $83.55(7.69)$ & & \\
\hline & Post & $\mathrm{PC}$ & $97.29(1.12)$ & $97.16(1.14)$ & & \\
\hline & & $\mathrm{NC}$ & $80.40(2.38)$ & $80.99(2.49)$ & & \\
\hline
\end{tabular}


Evaluation of a Correctional Substance Abuse Program 152

\begin{tabular}{lcccccc}
\hline Effect & & & Unadjusted & Adjusted & & \\
\hline Test time x group & & & $M(S E)$ & $M(S E)$ & $F$ & $\eta^{2}$ \\
PossitDTCQ & Pre & PC & $80.90(3.67)$ & $80.00(3.63)$ & 1.97 & .04 \\
& & NC & $72.80(7.78)$ & $76.87(7.92)$ & & \\
& Post & PC & $97.69(1.38)$ & $97.67(1.41)$ & & \\
& & NC & $81.20(2.92)$ & $81.27(3.06)$ & & \\
TempsitDTCQ & Pre & PC & $73.99(4.59)$ & $72.81(4.53)$ & 3.70 & .07 \\
& & NC & $64.13(9.74)$ & $69.48(9.87)$ & & \\
& Post & PC & $94.01(2.40)$ & $93.53(2.40)$ & & \\
& & NC & $67.40(5.09)$ & $69.59(5.24)$ & & \\
\hline
\end{tabular}

Note. Adjusted means evaluated at mean of covariate preprogram BIDR SDE subscale $=6.62$. Adjusted means evaluated at mean of covariate preprogram $B I D R I M$ subscale $=5.65$. Group $=$ program completers (PC) and noncompleters (NC); Test time = preprogram (Pre) and postprogram (Post); ConfCS = overall confidence in avoiding substance use measured by the client survey; NegsitDTCQ = confidence to not use substances in negative situations measured by the DTCQ; PossitDTCQ = confidence to not use substances in positive situations measured by the DTCQ; TempsitDTCQ = confidence to not use substances in temptation situations measured by the DTCQ. For program completers $n=45$ and $n=10$ for noncompleters.

${ }^{*} \mathrm{p}<.05{ }^{* *} \mathrm{p}<.01$. 
Hypothesis 6-Knowledge of Substance Abuse Issues Will Increase Following the Drug and Alcohol Education Component of the Program

Offender self-report. An ANCOVA was conducted to examine increases in knowledge of substance abuse issues following the drug and alcohol education component of the program using age as a covariate. In the postprogram client survey, offenders were asked how much their knowledge of substance abuse issues, such as shame, anger, denial, and addiction increased after watching the substance abuse videos. Results showed a significant effect for group, $F(1,22)=18.14, p<.001, \eta_{\mathrm{p}}^{2}=.45$, with a large effect size. That is, the program completers reported more of an increase in knowledge of substance abuse after the drug and alcohol education component of the program (adjusted $M=4.45, S E=.17$; unadjusted $M=4.25, S E=.17$ ) than noncompleters (adjusted $M=2.64, S E=.34$; unadjusted $M=2.60, S E=.34$ ).

In addition to using the client survey to measure changes in knowledge of substance abuse issues following the education component of the program, the combined video questionnaires designed to measure knowledge gained from the educational component were examined. An ANCOVA was conducted to assess the effects of the drug and alcohol education component of the program on knowledge of various substance abuse issues, with age and risk level as covariates. The between-subjects effect was group and the within-subjects effect was test administration time.

The result for the within-subjects effect of test administration time was significant, $F(1,21)=9.46, p<.01, \eta_{p}^{2}=.31$. Specifically, offenders had more knowledge of substance abuse issues after the education component of the program (adjusted $M=27.59, S E=.63$; unadjusted $M=27.58, S E=.64$ ) than before (adjusted $M$ 
$=24.96, S E=.74 ;$ unadjusted $M=24.91, S E=.77)$. The between-subjects effect was not significant, $F(1,21)=1.22, p=.28, \eta_{\mathrm{p}}^{2}=.06$, suggesting that the two groups' means scores did not significantly differ on knowledge. The interaction between test administration time and group was not significant, $F(1,21)=1.66, p=.21, \eta_{\mathrm{p}}^{2}=.07$.

Analyses of both the client survey and the combined questionnaires substantiate the original hypothesis, that is, the education component of the program increased offenders' knowledge of substance abuse issues. In general, offenders had more knowledge of substance abuse issues, measured by the combined video questionnaires, after participating in the education component than before their participation. From the perspective of the program participants, knowledge of substance abuse issues including anger, shame, denial, and addiction increased following their participation in the education program component.

Hypothesis 7-Motivation to Participate in Treatment Will Increase and Motivation Will Move Across the Stages Following the Initial Counselling Component

Offender self-report. In the postprogram client survey, offenders were asked how much their motivation to participate in the MDH program increased after the initial counselling component. Offenders who went through the original delivery of the program and offenders who went through the revised format were compared on this variable. An independent-samples $t$ test showed a significant difference between the two groups, $t(57)$ $=-2.19, p<.05$. Consequently, this new grouping of participants was used as an independent variable in further analyses. To examine increased motivation following the initial counselling, an ANCOVA was conducted with program completers and noncompleters using age and risk level as covariates. 
Results showed a significant difference between those offenders who went through the original program format and those who participated in the new format, $F(1$, $53)=8.78, p<.01, \eta_{p}^{2}=.14$, with the first group having lower means scores (adjusted $M$ $=2.98, S E=.27 ;$ unadjusted $M=2.99, S E=.30$ ) on motivation than the second group (adjusted $M=3.96, S E=.19$; unadjusted $M=3.93, S E=.21$ ). A significant difference was also found between program completers and noncompleters, $F(1,53)=12.05, p<$ $.01, \eta_{p}^{2}=.19$. Specifically, program completers had higher mean scores (adjusted $M=$ $4.06, S E=.15$; unadjusted $M=4.07, S E=.16$ ) on motivation than noncompleters (adjusted $M=2.88, S E=.30 ;$ unadjusted $M=2.85, S E=.33$ ).

The interaction between counselling format and program completion was not significant (see Table 22). Given the small-medium effect size as well as the interest in comparing the two different counselling formats, the profile plot was examined (see Appendix V, Figure V7). With the original counselling design, there was a large difference between the motivation levels reported by the groups, with the noncompleters reporting less motivation after the initial counselling component than the program completers. After changing the delivery format (i.e., adding counselling sessions), both groups reported higher levels of motivation. However, the noncompleters reported a larger increase in motivation than the completers. Nonetheless, after participation in the revised counselling activity, the difference between the two groups was reduced, with program completers and noncompleters reporting more similar increases in motivation (see Table 22).

To examine the effects of the initial counselling component on movement across the stages of change, the RCQ-TV was administered before and after the counselling 
Evaluation of a Correctional Substance Abuse Program 156

Table 22

Univariate Results from ANCOVA Examining Increased Motivation to Participate in

Treatment Following the Initial Counselling Activity Based on the Client Survey

\begin{tabular}{lcccc}
\hline & Unadjusted & Adjusted & & \\
Effect & $M(S E)$ & $M(S E)$ & $F$ & $\eta^{2} \mathrm{p}$ \\
\hline
\end{tabular}

Within-subjects effects

Delivery $\mathrm{x}$ group

$1.83 \quad .03$

Motivation

$\begin{array}{llll}\text { CounOR } & \text { PC } & 3.74(.25) & 3.80(.23) \\ & \text { NC } & 2.25(.54) & 2.16(.50) \\ \text { CounRV } & \text { PC } & 4.41(.21) & 4.32(.20) \\ & \text { NC } & 3.44(.36) & 3.60(.34)\end{array}$

Note. Adjusted means evaluated at mean of covariate age $=36.17$. Adjusted means evaluated at mean of covariate risk level $=2.27$. Delivery $=$ original delivery of the initial counselling session (CounOR) and revised delivery of the initial counselling session $($ CounRV $) ;$ Group = program completers $(\mathrm{PC})$ and noncompleters $(\mathrm{NC}) ;$ Motivation $=$ increases in motivation following the initial counselling session. For the original delivery of counselling group; $n=23$ ( $n=19$ for program completers and $n=4$ for noncompleters) and for the revised delivery of counselling group $n=36$ ( $n=27$ for program completers and $n=9$ for noncompleters). 
activity and a mixed ANOVA was conducted. The between-subjects effect was group and the within-subjects effect was test administration time. The result for the betweensubjects effect demonstrated a nonsignificant difference between the groups on motivation, $F(1,58)=.00, p=1.00, \eta_{\mathrm{p}}^{2}=.00$. In addition, the within-subjects effect was nonsignificant, $F(1,58)=1.32, p=.26, \eta_{p}^{2}=.02$. Examination of the means revealed that program participants were in the action stage of change $(M=2.99, S E=.02)$ prior to the initial counselling component as well as after $(M=2.94, S E=.03)$. Not surprisingly, the interaction between time and group was also nonsignificant, $F(1,58)=.33, p=.57, \eta_{\mathrm{p}}^{2}=$ .006 . The mean scores illustrated that both program completers and noncompleters were in the action stage of change $(M=2.98, S E=.02 ; M=3.00, S E=.03$, respectively) before the initial counselling component and after $(M=2.96, S E=.03 ; M=2.93, S E=$ .06 , respectively).

Given that lack of support for the original hypothesis with respect to movement across the stages of change following the initial counselling component, an additional analysis was conducted to explore whether participants' scores for each stage of change varied after the counselling activity as well as after the program. Specifically, a 2 (group: program completers and noncompleters) x 3 (test administration time: preprogram, after the initial counselling component, and postprogram) doubly multivariate repeated measures analysis of covariance was conducted using age and the preprogram BIDR IM as covariates. The between-subjects effect was group and the within-subjects effect was test administration time.

Using Wilks' $\lambda$, the multivariate results for the between-subjects effect showed a nonsignificant difference between the groups on the set of dependent variables, $F(3,34)$ 
$=1.27, p=.30, \eta_{\mathrm{p}}^{2}=.10$. A nonsignificant difference was found for the interaction between the test administration time and group, $F(6,140)=.32, p=.93, \eta_{p}^{2}=.01$. The multivariate results for the within-subjects effect was also nonsignificant, $F(6,140)=$ $1.59, p=.15, \eta_{\mathrm{p}}^{2}=.06$. However, given it was hypothesized that the initial counselling activity would increase motivation to change across time, the univariate results for the within-subjects effect were examined. The univariate results for each stage of change were nonsignificant with effect sizes in the small-medium to medium range (see Table 23). Examination of the within-subjects contrasts revealed a trend that provides some evidence of the effectiveness of counselling in increasing motivation to change substance-abusing behaviour. Specifically, scores on the contemplation subscale scores decreased after the initial counselling component and at posttest. In addition, the action subscale scores also decreased over time, with the largest decrease being after the initial counselling component to postprogram.

Program staff assessment. The staff survey required that program staff complete a subjective assessment of an offender's motivation to participate in the program as well as motivation to change their substance-abusing behaviour based on the categories of the RCQ-TV. Before beginning analysis, possible covariates were examined. Results revealed that age and the preprogram BIDR IM subscale scores were suitable covariates. However, it was determined that the preprogram BIDR SDE interacted with group, which violates the assumption of homogeneity of regression slopes; thus, MANCOVA was not suitable. Rather it was more appropriate to include the preprogram BIDR SDE as an independent variable in the design (i.e., Attribute by Treatment Interaction design). 
Evaluation of a Correctional Substance Abuse Program 159

Table 23

Univariate Results from MANCOVA Examining Motivation to Change Based on the $R C Q-T V$

\begin{tabular}{lcccc}
\hline & Unadjusted & Adjusted & & \\
Effect & $M(S E)$ & $M(S E)$ & $F$ & $\eta_{p}^{2}$ \\
\hline
\end{tabular}

Between-subjects effects

Group

Precontemplation $\quad \mathrm{PC}$

$\mathrm{NC}$

Contemplation

PC

NC

Action

PC

$\mathrm{NC}$

Within-subjects effects

Test time

Precontemplation

Contemplation

Action

est time $\mathrm{x}$ group

Precontemplation

$\mathrm{C}$

.

$-8.06(.44)$

$-7.99(.44)$

$1.54 \quad .04$

$-6.33(.88)$

$-6.63(.95)$

$4.12(.57)$

$3.97(.59)$

1.46

.04

$5.17(1.14)$

$5.74(1.28)$

$8.94(.29)$

$8.77(.28)$

.69

.02

7.54 (.58)

$8.20(.61)$

$-6.78(.61)$

$-7.09(.60)$

1.29

.04

$-7.56(.64)$

$-7.70(.69)$

$-7.25(.81)$

$-7.15(.84)$

Post

$4.69(.72)$

$5.19(.73)$

1.58

.04

$4.86(.77)$

$4.91(.82)$

$4.38(.89)$

4.47 (.96)

Post

$8.44(.37)$

$8.74(.37)$

1.80

.05

Mid

$8.20(.41)$

$8.58(.40)$

Post

$8.08(.53)$

$8.14(.55)$

\begin{tabular}{llllll} 
Pre & PC & $-8.19(.55)$ & $-7.98(.52)$ & .10 & .003 \\
& NC & $-5.38(1.09)$ & $-6.20(1.13)$ & & \\
Mid & PC & $-8.25(.57)$ & $-8.16(.60)$ & \\
& NC & $-6.88(1.14)$ & $-7.23(1.30)$ & \\
\multirow{2}{*}{ Post } & PC & $-7.75(.72)$ & $-7.82(.74)$ & \\
& NC & $-6.75(1.44)$ & $-6.47(1.59)$
\end{tabular}


Evaluation of a Correctional Substance Abuse Program 160

\begin{tabular}{|c|c|c|c|c|c|c|}
\hline Effect & & & $\begin{array}{c}\text { Unadjusted } \\
M(S E)\end{array}$ & $\begin{array}{c}\text { Adjusted } \\
M(S E)\end{array}$ & $F$ & $\eta_{p}^{2}$ \\
\hline \multirow[t]{6}{*}{ Contemplation } & Pre & $\mathrm{PC}$ & $4.25(.64)$ & $3.92(.63)$ & .27 & .007 \\
\hline & & $\mathrm{NC}$ & $5.13(1.28)$ & $6.45(1.37)$ & & \\
\hline & Mid & $\mathrm{PC}$ & $4.34(.69)$ & $4.31(.72)$ & & \\
\hline & & $\mathrm{NC}$ & $5.38(1.38)$ & $5.51(1.56)$ & & \\
\hline & Post & $\mathrm{PC}$ & $3.75(.80)$ & $3.69(.84)$ & & \\
\hline & & $\mathrm{NC}$ & $5.00(1.59)$ & $5.25(1.81)$ & & \\
\hline \multirow[t]{6}{*}{ Action } & Pre & $\mathrm{PC}$ & $9.13(.33)$ & $8.93(.32)$ & .44 & .01 \\
\hline & & $\mathrm{NC}$ & $7.75(.67)$ & $8.55(.70)$ & & \\
\hline & Mid & $\mathrm{PC}$ & $8.91(.37)$ & $8.66(.35)$ & & \\
\hline & & $\mathrm{NC}$ & $7.50(.74)$ & $8.50(.75)$ & & \\
\hline & Post & $\mathrm{PC}$ & $8.78(.47)$ & $8.74(.49)$ & & \\
\hline & & $\mathrm{NC}$ & $7.38(.95)$ & $7.54(1.05)$ & & \\
\hline
\end{tabular}

Note. Adjusted means evaluated at mean of covariate age $=36.48$. Adjusted means evaluated at mean of covariate preprogram BIDR IM subscale $=5.90$. Group $=$ program completers $(\mathrm{PC})$ and noncompleters $(\mathrm{NC})$; Test time = preprogram (Pre), after initial counselling component (Mid), and postprogram (Post). For program completers $n=32$ and $n=9$ for noncompleters. 
A 2 (group: program completers and noncompleters) x 2 (test administration time: program intake and program completion/departure) x 2 (BIDR SDE: at program intake and program completion/departure) doubly multivariate repeated measures analysis of covariance was conducted to assess the program staff's assessment of client motivation to participate in the program and motivation to change substance-abusing behaviour before and after the program. The between-subjects effects were group and preprogram BIDR SDE, and the within-subjects effect was test administration time.

Using Wilks' $\lambda$, the multivariate results for the between-subjects effect showed a significant difference between the groups on the set of dependent variables, $F(2,69)=$ $7.42, p<.01, \eta_{\mathrm{p}}^{2}=.18$. Univariate results demonstrated that the difference between the two groups was significant for the staff's assessment of motivation to participate in the program, with program completers being rated as having more motivation (see Table 24). In addition, significantly different ratings were found for motivation to change across the two groups, with program completers being assessed closer to the action stage of change and noncompleters being assessed closer to the contemplation stage of change (see Table 24).

The multivariate results for the between-subjects effect using Wilks' $\lambda$ showed a nonsignificant difference between the two levels of the preprogram BIDR SDE subscale on the set of the dependent variables, $F(2,69)=2.45, p=.09, \eta_{\mathrm{p}}^{2}=.07$. Furthermore, nonsignificant differences were found for the interaction between the preprogram BIDR SDE subscale and group, $F(2,69)=3.06, p=.05, \eta_{p}^{2}=.08$ as well as the test administration time by preprogram $\mathrm{BIDR} \operatorname{SDE}$ subscale interaction, $F(2,69)=1.69, p=$ $.19, \eta_{p}^{2}=.05$. 
Evaluation of a Correctional Substance Abuse Program 162

Table 24

Univariate Results from MANCOVA Examining Motivation to Participation in Treatment and Change Substance Abuse Behaviour Based on Program Staff Assessment

\begin{tabular}{lccccc}
\hline & Unadjusted & Adjusted & & \\
Effect & $M(S E)$ & $M(S E)$ & $F$ & $\eta_{\mathrm{p}}^{2}$ \\
\hline
\end{tabular}

Between-subjects effects

Group

MotivationSS

RCQ-SS

Within-subjects effects

Test time

MotivationSS

Test time $\mathrm{x}$ group

MotivationSS

RCQ-SS

Test time $x$ BIDR SDE

MotivationSS
PC

NC

PC

NC

Pre

Post

Pre

Post

Pre PC

$\mathrm{NC}$

Post PC

$\mathrm{NC}$

Pre PC

$\mathrm{NC}$

Post

PC

$\mathrm{NC}$

$\begin{array}{ll}\text { Pre } & \text { Moderate } \\ & \text { High } \\ \text { Post } & \text { Moderate }\end{array}$

High

$$
4.03(.12)
$$

$3.15(.14)$

$2.54(.06)$

$2.12(.07)$
$3.95(.12)$

$3.28(.15)$

$2.49(.06)$

$2.19(.07)$
$11.70^{* *} .14$

$9.98 * * \quad .13$

$3.68(.11)$

$3.70(.11)$

$.34 \quad .01$

$3.49(.12)$

$2.25(.07)$

$3.53(.12)$

$2.27(.06)$

$2.41(.05)$

$2.40(.05)$

$3.90(.14)$

$3.83(.14)$

6.60 *

.09

3.47 (.17)

$3.56(.18)$

$4.15(.16)$

$4.06(.16)$

$2.83(.19)$

$3.00(.20)$

$2.31(.08)$

$2.23(.08)$

$23.31^{* * *}$

.25

$2.20(.10)$

$2.30(.10)$

$2.78(.07)$

$2.75(.07)$

$2.03(.08)$

$2.08(.09)$

$3.70(.16)$

$3.77(.16)$

$3.41^{\dagger}$

.05

$3.63(.15)$

$3.77(.18)$

$3.88(.19)$

$3.22(.17)$

$3.18(.17)$ 
Evaluation of a Correctional Substance Abuse Program 163

\begin{tabular}{|c|c|c|c|c|c|c|}
\hline Effect & & & $\begin{array}{c}\text { Unadjusted } \\
M(S E)\end{array}$ & $\begin{array}{c}\text { Adjusted } \\
M(S E)\end{array}$ & $F$ & $\eta_{p}^{2}$ \\
\hline \multicolumn{7}{|c|}{ Test time $\mathrm{x}$ BIDR SDE } \\
\hline \multirow[t]{4}{*}{ RCQ-SS } & Pre & Moderate & $2.18(.10)$ & $2.27(.09)$ & .57 & .01 \\
\hline & & High & $2.32(.09)$ & $2.27(.08)$ & & \\
\hline & Post & Moderate & $2.43(.08)$ & $2.47(.08)$ & & \\
\hline & & High & $2.38(.07)$ & $2.36(.07)$ & & \\
\hline
\end{tabular}

Note. Adjusted means evaluated at mean of covariate age $=35.28$. Adjusted means evaluated at mean of covariate preprogram BIDR IM subscale $=5.63$. Group $=$ program completers $(\mathrm{PC})$ and noncompleters $(\mathrm{NC}) ;$ Test time $=$ preprogram $($ Pre $)$ and postprogram $($ Post $) ;$ BIDR SDE $=$ preprogram BIDR SDE subscale; MotivationSS = staff assessment of participant motivation to participate in the program; RCQ-SS $=$ staff subjective assessment of participant treatment readiness; Moderate = moderate score of the BIDR SDE; High $=$ high score on the BIDR SDE. For program completers $n=47$ and $n=29$ for noncompleters. ${ }^{\dagger} p<.10 .{ }^{*} p=.05 .{ }^{* *} p=.01 . * * * p=.001$ 
The test administration time by group interaction was significant on the set of dependent variables, $F(2,69)=11.85, p<.001, \eta_{p}^{2}=.26$. The univariate findings indicated that staff's ratings of motivation to participate in the program changed over time with different trends for each group of program participants. Specifically, staff rated program completers as having higher motivation at postprogram than at intake, whereas they assessed noncompleters with less motivation at program departure than at program intake (see Table 24).

In summary, the results provided partial support for the original hypothesis. Movement across the stages of change was not in the expected direction. Offenders did not move along the continuum of change. Indeed, they were in the action stage of change at program intake and at the mid assessment point. However, examination of offenders' scores for each stage of change revealed a promising trend. Specifically, contemplation scores decreased after the initial counselling component.

Interesting findings were discovered when the differences between the delivery formats of the initial counselling activity were examined. Those offenders who participated in the original counselling format (i.e., one initial session) reported lower levels of motivation than those offenders who participated in the revised counselling activity (i.e., more sessions added to the counselling component). In addition, noncompleters who participated in the original counselling format reported lower levels of motivation. However, with the revised format, both program completers and noncompleters reported similar levels of motivation.

Additional analyses conducted with the staff survey indicated that the subjective staff ratings of motivation to change substance-abusing behaviour appeared to be slightly 
more discriminating between program completers and noncompleters than the offender self-reported RCQ-TV results. Analyses of the subjective staff ratings of motivation to change detected a significant difference between the groups. That is, program completers were rated closer to the action stage of change in comparison to the noncompleters who were assessed closer to the contemplation stage of change. Program staff also rated program completers as having more motivation to participate in treatment from pretest to posttest than noncompleters.

Hypothesis 8-Knowledge of Prosocial Skills Will Increase Following the CognitiveBehavioural Activity

Offender self-report. The influence of the cognitive-behavioural activity on knowledge of prosocial skills was examined using the postprogram client survey, which asked offenders to rate how much their knowledge of various prosocial skills (i.e., assertiveness, anger, and problem-solving) increased after participating in the group sessions. One-sample $t$ tests were run on each prosocial skill variable. Bonferroni's correction was used to control for the number of tests (critical $\alpha=.017$ ). Results demonstrated significant differences for the three prosocial skills: assertiveness, $t(45)=$ $26.89, p<.001$, anger, $t(45)=20.71, p<.001$, and problem-solving, $t(45)=26.80, p<$ .001 . Program completers reported significant increases in knowledge of assertiveness $(M$ $=4.20, S D=.81)$, anger $(M=4.15, S D=1.03)$, and problem solving $(M=4.37, S D=$ .85). Therefore, the results supported the hypothesis that program participants who completed the full 8-week program would gain knowledge of assertiveness, anger, and problem-solving skills following the cognitive-behavioural activity. 
Hypothesis 9-Knowledge of Relapse Prevention Skills Will Increase Following the Cognitive-Behavioural Activity

Offender self-report. The impact of the cognitive-behavioural activity on knowledge of relapse prevention skills was examined using the postprogram client survey, which asked offenders to rate how much their knowledge of various relapse prevention skills (i.e., developing a relapse prevention plan, identifying high-risk situations, and using relapse prevention techniques) increased after participating in the group sessions. One-sample $t$ tests were run on each relapse prevention skill using Bonferroni's correction (critical $\alpha=.017$ ) to control for the number of tests. Results demonstrated significant differences for developing a relapse prevention plan, $t(45)=$ $27.73, p<.001$, identifying personal high-risk situations, $t(45)=29.73, p<.001$, and using relapse preventions techniques, $t(45)=31.74, p<.001$. Specifically, the analysis showed that program completers had an increase in knowledge of each relapse prevention skill following the cognitive-behavioural activity, which supports the original hypothesis.

Hypothesis 10-Ability with Prosocial Skills Will Increase Following the CognitiveBehavioural Activity

Offender self-report. The influence of the cognitive-behavioural activity on ability with prosocial skills was examined using the postprogram client survey, which asked offenders to rate how much their ability with various prosocial skills (i.e., assertiveness, anger, and problem-solving) increased after participating in the cognitive-behavioural group sessions. One-sample $t$ tests were run on each prosocial skill using Bonferroni's correction (critical $\alpha=.017$ ). Results demonstrated significant differences for assertiveness, $t(45)=25.22, p<.001$, anger, $t(45)=22.12, p<.001$, and problem 
solving, $t(45)=25.15, p<.001$. Thus, as hypothesized, the results revealed that program participants who completed the full 8 -week program gained ability to be assertive, deal with their anger, and problem solve following the cognitive-behavioural activity.

Hypothesis 11 - Ability with Relapse Prevention Skills Will Increase Following the Cognitive-Behavioural Activity

Offender self-report. Increased ability with relapse prevention skills following participation in the cognitive-behavioural activity was tested using the postprogram client survey, which asked offenders to rate how much their ability on various relapse prevention skills (i.e., developing a relapse prevention plan, identifying high-risk situations, and using relapse prevention techniques) increased after participating in the cognitive-behavioural activity. One-sample $t$ tests were run on each relapse prevention skill using Bonferroni's correction (critical $\alpha=.017$ ) to control for the number of tests. Results demonstrated significant differences for developing a relapse prevention plan, $t(45)=24.47, p<.001$, identifying personal high-risk situations, $t(45)=29.96, p<.001$, and using relapse preventions techniques, $t(45)=27.35, p<.001$. In summary, as expected the analysis demonstrated program participants who completed the full 8-week program had more ability to develop a relapse prevention plan, identify personal highrisk situations, and use relapse prevention techniques following the cognitive-behavioural activity.

Hypothesis 12 - Self-Improvement Following Cognitive-Behavioural Activity Offender self-report. A repeated measures analysis was conducted to examine self-improvement after participating in the cognitive-behavioural activity in comparison 
to the education and counselling program components, using age as a covariate. The analysis consisted of only those offenders who completed the entire 8-week program. The repeated measure was each component of the program.

Unlike the education component and counselling component of the program, the cognitive-behavioural activity was assessed in the client survey with several individual items related to prosocial and relapse prevention skills. A principal components analysis was conducted using a varimax rotation to reduce the number of variables associated with the cognitive-behavioural component for the repeated measures analysis. The results showed that the 12 items loaded on two factors (see Appendix U, Table U5). The new factors were named knowledge and ability with prosocial skills and knowledge and ability with relapse prevention skills. The internal consistency estimates for the knowledge and ability with prosocial skills and knowledge and ability with relapse prevention skills subscales were acceptable ( $\alpha=.94$ and $\alpha=.97$, respectively). Thus, it was decided that the analysis of hypothesis 12 would be conducted using two items from the postprogram client survey (i.e., the one item related to the education component of the program and the one item related to the counselling component of the program) and the two factors identified in the principal components analysis (i.e., knowledge and ability with prosocial skills and knowledge and ability with relapse prevention skills).

Using the Huynh-Feldt adjustment to correct for sphericity, the omnibus analysis indicated a significant difference across the program components, that is, selfimprovement varied across the program components (see Table 25). Follow-up comparisons were examined using the difference contrast. The comparisons revealed that the only significant difference was between the knowledge and ability with relapse 
Table 25

Results from Repeated Measures Analysis Examining Self-Improvement Following the Three Program Activities Measured Based on Offender Self-Report

\begin{tabular}{llllll}
\hline Effect & $M$ & $S E$ & $F$ & $\eta_{\mathrm{p}}^{2}$ \\
\hline
\end{tabular}

Within-subjects effects

Treatment component $4.35 * \quad .19$

Education 4.25

Counselling

Knowledge \& ability with prosocial skills

Knowledge \& ability with RP skills

Note. Adjusted means evaluated at mean of covariate age $=35.40$. The knowledge and ability with prosocial skills is associated with the cognitive-behavioural activity. The knowledge and ability with relapse prevention skills is also associated with the cognitivebehavioural activity program component. The sample size for each treatment component is 20 .

$* p=.05$. 
prevention skills cognitive-behavioural component of the program and the other program components combined, $F(1,18)=8.86, p<.01, \eta_{\mathrm{p}}^{2}=.33$. The offenders reported more self-improvement due to the relapse prevention component $(M=4.58, S E=.16)$ in comparison to the other program components combined $(M=4.11)$.

The knowledge and ability with prosocial skills cognitive-behavioural component of the program was not significantly different from the combined education and counselling components, $F(1,18)=1.50, p=.24, \eta^{2}=.08$; however, the effect size was medium-large. The low power of this test (.21) may be the reason for the nonsignificant finding. An examination of the mean scores showed that the offenders reported more selfimprovement from the prosocial skills cognitive-behavioural component $(M=4.27 ; S E=$ $.15)$ than from the education and counselling components combined $(M=4.03)$. The last contrast also indicated a nonsignificant difference between the education and counselling component of the program, $F(1,18)=4.34, p=.05, \eta_{p}^{2}=.33$, with a large effect size. Again, the low power of this test (.51) may be contributing to this nonsignificant finding. Indeed, more self-improvement was reported for the education component than for the counselling component (see Table 25).

Overall, the results demonstrated more benefit from the relapse prevention cognitive-behavioural program component in comparison to the other program components. Although comparisons are not significant between the education component, counselling component, and the prosocial cognitive-behavioural sessions component with respect to self-improvement among program completers, the magnitude of the effects are medium-large to large and the power ranges from small to moderate. This suggests that a larger sample may result in significant findings. 
Nonetheless, trends in the data suggest that the prosocial skills component had more of an impact on self-improvement than education or counselling. Even if these particular contrasts reached significance, the obtained mean of the relapse prevention skills component and associated effect size was considerably higher indicating that the component had the most impact on self-improvement. Thus, as hypothesized, it can be concluded that more self-improvement was produced by the cognitive-behavioural activity than either the education or counselling program components.

\section{Hypothesis 13 - Quality of Program Implementation}

Key informant interview. To assess the quality of program implementation, the integration of the principles of effective correctional treatment into the MDH program was examined. A checklist analysis was conducted using the notes taken on the responses provided by the Executive Director during the interview. For this analysis, the author coded the responses into one of three levels of adherence to each principle: (1) fully incorporated, (2) partially incorporated, and (3) not incorporated (see Appendix U, Table U6). Of the 15 principles covered in the interview, the program fully adheres to 11 .

As can be seen from Appendix U, Table U6, two of the three original principles of effective correctional treatment are not entirely incorporated into the program. First, for the risk principle, the large majority of offenders accepted into the program are medium to high risk. However, in contrast to the theory of the risk principle, the program does accept a small number of offenders who are low risk, when MDH assesses an offender as having high need for substance abuse treatment. Second, for the responsivity principle, the general responsivity principle is fully incorporated into the program, but the specific responsivity principle is just partially incorporated. The rationale for this partial 
rating was that although $\mathrm{MDH}$ recognizes the value of the specific responsivity principle, operational considerations, such as security of staff and caseloads may take priority over ensuring offender characteristics are matched with the characteristics of his counsellor.

Another principle partially integrated into the $\mathrm{MDH}$ program is related to staff qualifications, that is, the recommendation that staff participate in 3- to 6-months of formal and on-the-job training. At the present time, program staff at MDH receive approximately 1 -month of formal and on-the-job training a year. The only principle not integrated into the MDH program was delivery of the intervention over a 3- to 9-month period. Currently, MDH is funded for its 8-week program.

\section{Additional Analyses - Prediction of Program Completion}

Given that many of the hypotheses in this evaluation investigated differences between program completers and noncompleters, it was of further interest to examine client variables that may predict program completion. Predictors were selected based on previous research in the evaluation literature. Stevens (2002) indicates that generally 15 subjects per predictor are needed for a reliable regression equation in the social sciences. Since the total sample for this analysis was 58 , four predictors were selected. Due to the exploratory nature of this analysis and the low power associated with the current sample size, it was decided to use a more liberal alpha $(.10)$ for the tests of significance that follow.

A direct logistic regression analysis was performed on program completion as the outcome and the number of previous community substance abuse treatment programs completed, risk level, substance used most often, and age as the predictors. A test of the full model with the four predictors against a constant-only model was statistically 
significant, $\chi^{2}(5, N=58)=14.17, p<.05$, indicating that the predictors improved the ability to predict program completion. Using a pseudo $R^{2}$ measure, the results showed that the prediction of program completion was modest knowing the set of predictors (Nagelkerke $R^{2}=.29$ ). Table 26 presents a summary of the logistic regression analysis.

According to the Wald criterion, the number of previous community substance abuse treatment programs an offender completed significantly predicted program completion with all other predictors held constant (see Table 26). Thus, the odds of completing the program were 1.53 higher with an increase of one completed prior community substance abuse program, after statistically controlling for the other predictors. Although risk level overall was not significant, results for the contrasts between risk levels showed interesting odds. The odds for program completion among medium risk offenders were 1.93 times higher than for high risk offenders. The results also showed significant odds for program completion with low risk offenders having 7.34 times higher odds than high risk offenders. An examination of the substance used most often as a predictor revealed that offenders who used alcohol most often had a 5.13 higher odds of completing the program than offenders who used drugs most often, after statistically controlling for the other predictors. Finally, findings showed that offenders age 35 and above had a 2.32 higher odds of completing the program in comparison to offenders age 20 to 34 , with all other predictors held constant.

The classification results were examined next from the logistic regression analysis. The classification cutoff was changed from .5 (default) to .6 . This change was made to more accurately reflect correctional program completion rates. It should be noted 
Evaluation of a Correctional Substance Abuse Program 174

Table 26

Summary of Direct Logistic Regression Analysis for Variables Predicting Program

Completion $(n=58)$

\begin{tabular}{|c|c|c|c|c|c|c|}
\hline \multirow[b]{2}{*}{ Variable } & \multirow[b]{2}{*}{$B$} & \multirow[b]{2}{*}{$S E$} & \multirow[b]{2}{*}{$t$} & \multirow[b]{2}{*}{$\operatorname{Exp}(B)$} & \multicolumn{2}{|c|}{$95 \%$ CI for $\operatorname{Exp}(B)$} \\
\hline & & & & & Lower & Upper \\
\hline PrvCmTx & .42 & .25 & $1.72^{\dagger}$ & 1.53 & .94 & 2.47 \\
\hline Risk level & & & 1.75 & & & \\
\hline [High risk] & & & & & & \\
\hline Medium risk & .66 & .64 & 1.02 & 1.93 & .55 & 6.81 \\
\hline Low risk & 1.99 & 1.21 & $1.65^{\dagger}$ & 7.34 & .69 & 78.03 \\
\hline
\end{tabular}

Substance used most

[Alcohol]

$\begin{array}{lllllll}\text { Drugs } & 1.63 & 1.18 & 1.38 & 5.13 & .50 & 52.25\end{array}$

Current age

[Age 35 and above]

Age 20 to 34

.84

$.62 \quad 1.36$

2.32

.69

7.80

Note. [Brackets] indicate reference category. $\operatorname{PrvCmTx}=$ Number of previous community substance abuse treatment program completed.

$\dagger^{\dagger} p<.10$. 
that using a cutoff of .6 is likely conservative, given that a typical program completion rate for offender treatment programs is $75.0 \%$ (Lösel, 2001). The results of the current study demonstrated an $80.0 \%$ correct prediction rate for program noncompletion and a $63.6 \%$ correct prediction rate for program completion. Thus, the overall correct prediction rate for this study was $70.7 \%$. It is likely that the obtained classification results are an overestimate because according to Pedhazur (1997) predicted probabilities based on an equation derived from the same data tend to be inflated. Therefore, a discriminant function analysis (DFA) was conducted for cross-validation purposes.

Consistent with the reasoning for changing the logistic regression cutoff, it was decided to compute the prior probabilities from the group sizes instead of using the default (i.e., all groups equal) in the DFA. Table 27 presents the classification results using the DFA. As can be seen, the overall correct classification rate $(69.0 \%)$ was slightly lower than the classification rate obtained using the logistic regression $(70.7 \%)$. The results using the jackknife procedure were slightly lower than the original classification in the DFA, with $67.2 \%$ of cross-validated cases being classified correctly. Based on the small difference between the original classification results and the cross-validated results, it can be concluded that the results using this set of predictors would likely replicate using another sample.

In summary, it appears that program completion can be successfully predicted from age, risk level, number of previously completed community substance abuse programs, and substance used most often. In addition, the magnitudes of the odds ratios suggest that the best unique predictors are risk level, substance used most often, and age. Finally, the classification results indicate that with using this set of predictors, a 
Evaluation of a Correctional Substance Abuse Program 176

Table 27

Classification Results Using Discriminant Function Analysis $(n=58)$

\begin{tabular}{|c|c|c|c|}
\hline & \multirow[b]{2}{*}{ Program completed } & \multicolumn{2}{|c|}{ Predicted group membership } \\
\hline & & No & Yes \\
\hline \multicolumn{4}{|c|}{ Original } \\
\hline \multirow[t]{2}{*}{ Count } & No & 17 & 8 \\
\hline & Yes & 10 & 23 \\
\hline \multirow[t]{2}{*}{ Percentage } & No & 68.0 & 32.0 \\
\hline & Yes & 30.3 & 69.7 \\
\hline
\end{tabular}

Cross-validated

$\begin{array}{lccc}\text { Count } & \text { No } & 17 & 8 \\ & \text { Yes } & 11 & 22 \\ \text { Percentage } & \text { No } & 68.0 & 32.0 \\ & \text { Yes } & 33.3 & 66.7\end{array}$

Note. $69.0 \%$ of original grouped cases correctly classified; $67.2 \%$ of cross-validated grouped cases correctly classified. 
Evaluation of a Correctional Substance Abuse Program 177

substantial proportion of completion and noncompletion cases can be correctly classified.

Moreover, the regression equation is likely to cross-validate using another sample.

Reproduced with permission of the copyright owner. Further reproduction prohibited without permission. 
A significant number of first-generation evaluations of correctional substance abuse programs have been completed, and interest continues in this area. Secondgeneration evaluations tend to be more comprehensive as they include additional program outcomes. The current evaluation makes two important contributions to the existing second-generation evaluation literature. First, this evaluation used the program's theory to guide the evaluation design. This approach not only produced results that explain more about the utility of individual program activities and the overall program, but also generated findings that could be applied to program improvement. Second, by examining outcomes other than the ultimate program outcomes of recidivism and relapse, the present evaluation provided additional information on the personal development of offenders due to their participation in a substance abuse treatment program.

\section{Summary of Findings and Theoretical Linkages}

Knowledge and ability with prosocial and relapse prevention skills. It was hypothesized that offenders who completed the MDH program would have a greater increase in their knowledge and ability with prosocial skills in comparison to offenders who did not finish the program. The knowledge hypothesis was supported. Indeed, program completers reported more of an increase in knowledge of assertiveness, anger management, and problem-solving skills than noncompleters. These results were corroborated by the professional opinion of program staff. Specifically, counsellors reported that offenders who completed the program had more knowledge of all three prosocial skills than offenders who did not complete the program. The influence of personal bias in the staff assessments is believed to be minimal, since program staff are 
required to have extensive training and experience in counselling. Thus, it is assumed that the counsellors are aware of potential personal bias and that they try to ensure their personal feelings do not influence their professional judgement. The offender self-reports and staff assessments extend prior research that examined knowledge gain only among program completers (Millson et al., 1995; $\mathrm{T}^{3}$ Associates, 1999b, 1999c) to show that program completers have a greater gain in knowledge of prosocial skills than noncompleters.

Knowledge of prosocial skills appeared to be influenced by risk level. Results showed that knowledge of prosocial skills was not greatly increased among low risk offenders. In contrast, medium and high risk offenders illustrated substantial knowledge gain in specific prosocial skill areas. These findings tend to support the risk principle, which suggests treatment is most successful when its intensity is matched with risk level (Andrews \& Bonta, 2003; Andrews, Zinger, et al., 1990; Farabee et al., 1999; McGuire \& Priestly, 1995).

The ability hypothesis was partially supported by the current evaluation. In general, program completers did not report more of an increase in ability with prosocial skills than noncompleters. On the other hand, program staff reported group differences in ability with prosocial skills. Specifically, counsellors reported that program completers had more ability with anger management and problem-solving skills in comparison to noncompleters.

Program completion was hypothesized to result in more knowledge and ability with relapse prevention skills. The findings from the evaluation supported this hypothesis. With respect to knowledge of these skills, the two groups were not 
statistically different; however, the expected trend was evident with program completers reporting a greater increase in knowledge than noncompleters. Results revealed that ability with relapse prevention skills increased as a function of program completion. The professional opinion of program staff supported these two findings. These results on knowledge gain are consistent with past research (Millson \& Robinson, 1992).

Overall, the results indicated that substance-abusing offenders who participated in the MDH program gained knowledge and ability related to both prosocial and relapse prevention skills, skills that have been shown to assist individuals in changing their substance-abusing behaviour. These findings support the applicability of social learning theory in the construction of substance abuse programming. The underlying premise of social learning theory is that behaviours are largely learned; thus, maladaptive behaviours can be changed by learning cognitive and behavioural skills (Bandura, 1977).

Confidence to not use substances. At the outset of this evaluation, it was hypothesized that program completers would have a greater increase in their confidence to not use substances in comparison to noncompleters. Results validated this hypothesis. Program completers exhibited more overall confidence than noncompleters. In addition, offenders who graduated from MDH had more confidence in negative situations than offenders who did not graduate. Furthermore, trends seemed to indicate the same pattern for positive and temptation situations. These results augment past research that examined confidence among only those offenders who completed substance abuse programming (Peters et al., 1993) by illustrating that program completers gain more confidence than noncompleters. 
Influence of the drug and alcohol education activity on knowledge of substance abuse issues. It was hypothesized that the drug and alcohol education component of the program would increase knowledge of substance abuse issues. Results generated from participant opinion and the video questionnaires supported this hypothesis. Offenders not only reported more knowledge of shame, addiction, anger, and denial after participating in the education activity than before participation, but they also had higher scores on the video questionnaires after the education component than before. These findings are consistent with previous studies that have examined knowledge gain resulting from drug and alcohol education programs (Baldwin, 1990; Correctional Service of Canada, 1996a). Furthermore, the current evaluation findings are consistent with prior research on knowledge gain resulting from viewing videos that have shown watching educational videos increased knowledge among alcohol abusers (Miller \& Hester, 1986).

Influence of the counselling activity on motivation to participate in treatment and motivation to change substance abuse behaviour. The initial counselling component was expected to increase motivation among program participants. Results provided partial support for this hypothesis. Examination of the RCQ-TV revealed that offenders could not progress to the next stage of change after the initial counselling component. At pretest, offenders were already in the final stage of change (i.e., the action stage). Thus, no stage is further along the continuum for offenders to move to following the counselling component.

This finding seems to contradict the transtheoretical model that asserts individuals participating in treatment progress through stages of change. However, the observed result may be stemming from the RCQ-TV's inability to accurately assess the stage of 
change among offenders who are recently released from prison. The institutional environment does not produce the same type of demands as living in the community and the drug supply may be more limited. Consequently, while incarcerated, the substance abuse patterns of an offender may diminish. Thus, upon release, the offender may have a false sense of his ability to deal with his substance abuse problem. An offender's misguided belief of being capable of managing his substance abuse problem may be captured as the action stage of change by the RCQ-TV.

Legal coercion may be another reason why the offenders participating in the MDH program were assessed in the action stage at program intake. Most of the MDH participants have a parole condition that obligates them to participate in a substance abuse treatment program upon release. This requirement enforced by the National Parole Board may influence the results of the RCQ-TV. Gregoire and Burke (2004) found that among a group of substance abuse program participants, those participants who entered treatment because the legal system required them to had more motivation to change than those participants who enrolled in treatment for other reasons.

Further examination of the actual means scores for the stages of change revealed that the mean score for the action stage was lower after the initial counselling component than before. This finding is in opposition to the study by Vanderburg (2002) that revealed increased action scores from pretest to posttest among drug-abusing offenders exposed to one motivational interviewing session. A possible explanation for the more conservative action score in the current evaluation is that the offenders participated in a greater number of motivational interviewing sessions, which could bave provided offenders with more time to discuss and think about their substance-abusing behaviour. The extra time spent 
on self-reflection may have resulted in offenders scoring lower on the action scale at posttest.

The program staff's subjective interpretation of the stage of change utilized more of the continuum of change, unlike the RCQ-TV, which located offenders in the action stage at each of the three test administration times. Even though at pretest both completers and noncompleters were assessed by the counsellors as being close to the contemplation stage of change, at posttest completers were reported as being close to the action stage, while the noncompleters remained in contemplation.

Examination of offenders' scores for each stage of change revealed a promising trend, which lends support to the original hypothesis. Specifically, after attending the initial counselling component, offenders had lower scores for the contemplation stage than before participating in the counselling component. This finding is similar to past research, in which a decrease in contemplation scores was documented and interpreted as increased engagement in therapy (Prochaska et al., 1992). Other research using the RCQTV and SOCRATES have captured movement within individual stage of change scores after participation in a motivational interviewing session (Ginsburg, 2000; Vanderburg, 2002). ${ }^{24}$ The present finding supports the theory that motivational interviewing can prepare individuals for treatment by increasing problem recognition and planning for action (DiClemente \& Velasquez, 2002; Miller \& Rollnick, 2002; Zweben \& Zuckoff, 2002).

24 The Stage of Change Readiness and Treatment Eagerness Scale (SOCRATES), like the RCQ-TV, measures readiness to change. This instrument is composed of three scales: recognition, ambivalence, and taking steps. The recognition scale resembles the precontemplation scale of the RCQ-TV. The ambivalence scale corresponds to the contemplation scale of the RCQ-TV. The taking steps scale resembles the action scale of the RCQ-TV. 
Investigation of the two different delivery formats for the counselling activity revealed interesting results. First, in general, offenders reported increased motivation to participate in treatment after involvement in the counselling activity. This finding is similar to the study conducted by Peters et al. (1993), in which offenders reported improved motivation after participating in six months of a substance abuse program. Second, the original counselling format (i.e., one initial session) produced lower levels of motivation to participate in treatment than the revised counselling format (i.e., more sessions added to the initial counselling session). Third, the revised counselling design seemed to equate program completers and noncompleters on their reported levels of motivation to participate in the program. The latter two findings suggest that the redesign of the initial counselling session enhanced the activity's ability to influence offender motivation.

\section{Influence of the cognitive-behavioural activity on knowledge and ability of} prosocial and relapse prevention skills. It was hypothesized that knowledge and ability with prosocial and relapse prevention skills would increase following participation in the cognitive-behavioural activity. This hypothesis was supported by the results of the current evaluation. Offenders who completed the entire program reported that their involvement in the cognitive-behavioural activity increased their knowledge and ability with various prosocial skills (i.e., assertiveness, anger management, and problemsolving). These offenders also reported more knowledge and ability with developing a relapse prevention plan, identifying high-risk situations, and using relapse prevention techniques. The gain in knowledge of prosocial and relapse prevention skills following participation in a cognitive-behavioural substance abuse treatment program is consistent 
with past research (Millson et al., 1995; Millson \& Robinson, 1992; Peters et al., 1993; T³ Associates, 1999b, 1999c).

Self-improvement following the cognitive-behavioural activity. Offenders who completed the program were expected to report more self-improvement following the cognitive-behavioural activity than the other two program activities. This hypothesis was validated. In comparison to the education and counselling program components, offenders felt that they experienced more personal gain from the cognitive-behavioural activity. Offenders perceived the most self-improvement from the cognitive-behavioural sessions that focused on relapse prevention.

This finding is consistent with the general responsivity principle (Andrews \& Bonta, 1998, 2003). This principle, which is based on the general personality and social psychological perspective, asserts that the learning style of offenders needs to be considered in the design of programming intended to reduce recidivism. Furthermore, it is recognized that cognitive-behavioural programming is the most appropriate treatment approach for offender populations because it facilitates their learning (Andrews, 1989; McGuire \& Priestly, 1995).

Adherence to the principles of effective correctional treatment. An interest of this evaluation was to assess the quality of program implementation, as few studies have examined this issue in the past. More specifically, this assessment was designed to determine whether the MDH program adhered to the principles of effective correctional treatment. A checklist of several principles was created, and the majority of the principles were found to have been fully incorporated into MDH's design and delivery. Three of the four remaining principles were partially incorporated into the program. Only one 
principle was not adhered to (i.e., that the program is delivered over a 3- to 9-month period). The MDH program is delivered over a 2-month period. From this assessment, MDH can be described as an appropriate treatment for offenders.

This finding is similar to the conclusion of a recent program review of $\mathrm{MDH}$ (Orbis, 2002). For this review, the program was assessed against the Correctional Service of Canada's program accreditation criteria in preparation for consultation with an independent panel of experts. The report concluded that MDH was complying with a number of the critical accreditation requirements.

Prediction of program completion. From additional analyses it appears that several offender characteristics predict completion of the MDH program. Results indicated that previous completion of community substance abuse treatment, substance used most often, risk level, and age of the offender predicted program completion. Of the four predictors, the best individual predictors were being lower risk, using alcohol rather than drugs, and being 35 years old or older. Thus, an offender with any of these three characteristics had higher odds of completing the program. The finding that older offenders had higher odds of finishing treatment is consistent with the research completed by Knight and Hiller (1997).

Taken together, these findings suggest that the MDH program is meeting its shortand long-term objectives. Each program activity produced its intended short-term outcome. Specifically, the education component increased knowledge of substance abuse issues, the counselling activity increased motivation, and the cognitive-behavioural activity increased knowledge. In addition, confidence to not use substances and ability 
with prosocial and relapse prevention skills were improved after participation in the program.

\section{Implications and Recommendations}

The information and experience generated from this evaluation leads to recommendations not only for the $\mathrm{MDH}$ program, but also for funding agencies that resource community substance abuse treatment programs and the correctional evaluation community.

$M D H$ program. The results of this evaluation showed that the increased number of counselling sessions that incorporate motivational interviewing techniques appear to produce more motivation than the original one session format. Furthermore, this redesign is equating program completers and noncompleters on their reported level of motivation. Specifically, offenders not completing the program are now reporting levels of motivation that are more similar to program completers than when they were involved in the original design. These findings indicate that the redesign of the initial counselling session was an appropriate program modification. Thus, it seems to be in the best interest of the program and its clientele to continue using this program structure.

The findings from the examination of the RCQ-TV were not overly promising with respect to administering the instrument to track movement across the stages of change among an offender sample. Nonetheless, the RCQ-TV does appear to be able to assess movement within the stages of change. These changes in scores can be utilized for counselling purposes given specific motivational interviewing techniques exist that should be used with each particular stage (DiClemente \& Velasquez, 2002). That is, assessment of change in scores allows a counsellor to understand where an offender is in 
the process of change and accordingly, the counsellor can offer the most appropriate assistance. Therefore, it is recommended that the RCQ-TV remain a part of the preprogram and postcounselling test batteries not only to inform counsellors on the progress their clients are making within all stages of change, but also to inform the tailoring of their counselling practices with each client.

Even though other instruments exist that measure stage of change, it is recommended that $\mathrm{MDH}$ continue to implement the RCQ-TV. The rationale for this suggestion is twofold. First, the RCQ-TV has produced the most consistent results with substance-abusing offenders in recent studies. These studies have demonstrated the instrument's ability to assess movement within the stages of change (Ginsburg, 2000; Vanderburg, 2002). Second, studies have found that the URICA is not able to track movement across or within the various stages of change among substance-abusing offenders, and the SOCRATES has demonstrated mixed results with this population (Ginsburg, 2000; Vanderburg, 2002).

Examination of the cognitive-behavioural component of the $\mathrm{MDH}$ program demonstrated that offenders find this program activity increases their knowledge and ability with prosocial and relapse prevention skills. However, some evidence suggests that offenders are not fully developing their ability with prosocial skills. This is an interesting finding, given that almost exactly the same amount of time is spent on prosocial and relapse prevention skills in the cognitive-behavioural sessions. Inspection of the delivery format of the relapse prevention sessions reveals that more practicing (i.e., role-playing) is incorporated into these sessions than the prosocial skills sessions. This practice may be influencing the results, as offenders may perceive more personal 
development from the actual application of the relapse prevention skills. Therefore, it is recommended that $\mathrm{MDH}$ consider incorporating more role-playing into the prosocial skills component of the cognitive-behavioural activity.

Given the suggestion in the literature that cognitive-behavioural interventions should focus on social skill and relapse prevention as well as the evidence that this type of intervention changes behaviour among offenders, MDH may want to increase the amount of time spent on these two skill areas. Furthermore, MDH may want to examine ways to extend the length of the program over the suggested 3- to 9-month period. Increasing the length of stay at MDH would provide offenders with more time to further solidify their new skills.

The demographic information on risk level shows that $11 \%$ of the total sample in the evaluation was low risk. Past research has indicated that the best program outcomes are attained when treatment intensity is matched with risk level (Andrews \& Bonta, 2003; Andrews, Zinger et al., 1990; Bonta, 1996; Gendreau, 1996; Farabee et al., 1999; McGuire \& Priestley, 1995). Indeed, research has shown that when intensive services were provided to low risk offenders, they either had a very minimal effect or a negative effect, that is, increased recidivism (Andrews \& Bonta, 2003). Although recidivism was not examined in the present evaluation, low risk offenders may have minimal success with respect to recidivism in comparison to medium and high risk offenders since MDH is considered a medium to high intensity program. In light of the potential for a treatment by risk interaction, $\mathrm{MDH}$ should be mindful of targeting medium to high risk offenders.

Funding agencies. The existing literature has identified ongoing evaluation as a principle of effective correctional treatment, and continuous evaluation is an accreditation 
criterion for the Correctional Service of Canada. Even with the importance placed on program evaluation, community-based substance abuse programs, like $\mathrm{MDH}$, have difficulty completing evaluations. The inability to meet the expectation of ongoing evaluation stems from limited financial resources and research capacity. Often the funds received by a program are just enough to ensure the program is delivered to its clientele. Consequently, it is recommended that funding agencies, in particular those that require programs to adhere to the principles of effective correctional treatment allocate additional funding to permit ongoing evaluation and the contracting of qualified evaluators.

It is evident from the literature that the Correctional Service of Canada is committed to accrediting community substance abuse programs. Essentially, accreditation involves the assessment of programs based on evidence of what works in correctional settings. However, it is recognized that the accreditation process currently being used requires human resource skills and budgets usually beyond those found within the community setting (Bonta \& Cormier, 1999). Thus, if an appetite exists for developing a more accessible mechanism to assess adherence to the principles of effective correctional treatment, funding agencies could consider developing a checklist similar to the one applied in the current evaluation. Although this type of checklist format may not be as rigorous as the accreditation process or the CPAI, it still provides valuable information on the quality of a program while remaining cost-effective for all parties involved.

Correctional program evaluators. The results from the current evaluation underscore the utility of program evaluation for program monitoring and informing decisions related to program improvement. The primary reason this evaluation has 
practical application for program monitoring and improvement is the fact that the evaluation design was based on the program's theory. Incorporation of the program theory into the methodology ensured that the evaluation examined outcomes relevant to the program. Therefore, it is strongly encouraged that evaluators follow the same process for designing program evaluations. The aforementioned suggestion does not imply that evaluation preclude examination of ultimate outcomes, such as recidivism and relapse, particularly if these outcomes are part of the program's theory. As with the short- and long-term outcomes examined in the present evaluation, information on recidivism and relapse provides programs and funding agencies with compelling indicators of program success.

\section{Limitations of the Evaluation}

As with most field research, the present evaluation has a few shortcomings. These limitations relate to the design of the evaluation, the collection of data, the use of selfreport instruments, and program staff bias.

Evaluation design. The use of a comparison group versus a true randomized control group is frequently considered a weakness of an evaluation design. Often the experimental design is touted as the best design to use in conducting research because it controls for both known and unknown potentially confounding variables. For this particular program evaluation, a randomized control group could not be constructed because all of the offenders who reside at MDH participated in the program. The use of a comparison group, however, means that the quasi-experimental design used in this evaluation does lend itself to accurate conclusions for several reasons: (1) the outcomes measured were specific to the MDH program and thus, unlikely that the attainment of the 
outcomes would occur outside the program; (2) most of the outcomes measured were short-term, which are not likely to change without the substance abuse intervention; and (3) having offenders reside at $\mathrm{MDH}$ during program participation reduces the likelihood that the outcomes were influenced by factors other than the program itself.

A further limitation of the current evaluation relates to the issue of sample size. Small samples have limited statistical power and as a result, statistically significant results can be infrequent. This problem is evident in the current evaluation. Calculating the sample size necessary for adequate statistical power prior to beginning a study is a common guideline for statistical testing. In social science research where small-medium to medium effect sizes are more the norm, larger sample sizes are necessary for adequate power to detect differences. For instance, when looking at mean differences, to have sufficient power $(.80)$ to detect a medium effect size using an alpha $=.05$, each group needs to consist of 64 participants. This group sample size translates into a total sample size of 128 . In the same analysis situation, the sample size for each group must increase to 393, if the researcher is trying to detect a small effect size (Cohen, 1992). Thus, for studies expecting small-medium to medium effect sizes, the required sample size for adequate power is often difficult to achieve.

In the current evaluation, data were collected over a 16-month period to obtain the proposed sample of 80 offenders. At the onset of the evaluation, it was recognized that attaining a large sample would be require a substantial investment of time due to the average annual participation rate at $\mathrm{MDH}$. An average of 70 offenders reside at $\mathrm{MDH}$ over the course of one year. This low participation rate is a reflection of the limited bed space within the half-way house. A larger sample than the one used in this evaluation 
would have required data collection over a number of years. Nonetheless, the current sample size adheres to the Central Limit Theorem, which suggests that a sample of 30 or more tends to be large enough to approximate a normal distribution (Labute, 2002; Ramirez, 1995).

In addition, it should be noted that the present evaluation compensated for the small sample size by utilizing a repeated measures design. The advantage of a repeated measures design is that variability due to individual differences is completely removed from the error term; hence, power is increased (Stevens, 2002). In fact, Stevens states that this property of the repeated measures design makes it more powerful than completely randomized designs where different participants are randomly assigned to the different treatments. Furthermore, given that the same participants are being used repeatedly, far fewer participants are required for the study. Although the use of a repeated measures design compensated for the small sample size to a certain degree with respect to particular analyses, it is likely that low power remained an issue in analyses that had very few participants per cell.

Data collection. With respect to the administration of the pre- and postprogram battery, counsellors provided the questionnaires to offenders with instructions to complete the tests by the next day and offenders were told that if they had questions their counsellor was available to assist them. This test administration procedure allowed for offenders to complete the pre- and postprogram batteries without continuous counsellor supervision. Due to this test administration procedure, offenders may have obtained inaccurate guidance from other offenders in the house while completing the questionnaires. However, the counsellors reported that most offenders came to see them 
with questions. Therefore, in addition to the possible inaccurate guidance from other offenders, the majority of offenders were receiving proper guidance in the completion of the pre- and postprogram questionnaires from their counsellor.

Moreover, when an offender departed from the program early, counsellors were supposed to complete the postprogram staff survey. Due to the priority of other office procedures, counsellors were not always able to complete the postprogram staff survey at departure. In a few instances, the survey was completed several weeks later. This lag in response time could have resulted in inaccurate recall of the offender's performance.

On the other hand, completion of the survey at departure could be viewed as problematic. A parole violation is often the reason for early program departure. At MDH, the most common parole violation is using substances. This negative situation could bias a counsellor's recall of offender performance resulting in deflated ratings. In the current evaluation, the influence of personal bias is believed to be minimal, since program staff are required to have extensive training and experience in counselling. Thus, it is assumed that the counsellors are aware of potential personal bias and that they try to ensure their personal feelings do not interfere with their professional judgement.

An additional limitation relates to the administration of the postvideo questionnaires. The postvideo questionnaires were administered to offenders two to four days after watching the mandatory educational videos. This administration schedule was based on the length of the counselling activity, which varied from three to five days. Due to this program arrangement, it was not possible to have a standardized time for the administration of the postvideo questionnaires and thus, participants varied on the number of days in between test administrations. Consequently, it may be argued that the 
data collected after two days may be tapping memory recall versus knowledge of substance abuse issues. Nonetheless, in the current evaluation, the shortest length of time between the administration of the pre- and postvideo questionnaires (i.e., two days) was considered long enough to measure knowledge.

Self-report instruments. A final limitation is that the bulk of the analyses in this evaluation relied on offender self-report. However, past research completed by the CSC provides support for the conclusion that substance-abusing offender self-report data is reliable and valid (Beal et al., 1996; Weekes et al., 1997). Furthermore, in this evaluation several hypotheses were tested using offender self-report and counsellor assessments. Using multiple data sources allowed for the triangulation of results. In most instances in the present evaluation, the professional opinion of the program staff corroborated the reports of the offenders. Possible demand characteristics influencing offenders' selfreports were also taken into consideration by administering the BIDR to measure socially desirable responding. Where deemed appropriate, the subscales of the BIDR were used as a covariate in the statistical analyses.

Program staff bias. Even though the program staff have extensive professional training and experience as counsellors, the data collected from the postprogram staff assessments are still susceptible to personal bias. For example, when completing the postprogram assessment, counsellors have access to their preprogram assessments. Therefore, with this knowledge of preprogram scores, some bias could have entered into the data. In addition, counsellors may have a personal interest in the success of the program and/or individual offenders, and consequently, this interest may positively influence their assessments. 
Future Program Evaluation and Research Directions

Based on the present evaluation and review of the literature, possible areas of examination in future evaluations of $\mathrm{MDH}$ are suggested. As well, future directions for research in the area correctional substance abuse programming are proposed.

Future evaluations of $M D H$. In the current evaluation, several analyses compared program completers and noncompleters. The noncompleter group consisted of offenders who did not finish the entire 8-week program. On average, this group completed 3.5 weeks of the program, with a range from less than one week to seven weeks. Given the varying lengths of program involvement among the noncompleters, any future evaluation of MDH could consider constructing comparison groups derived from the length of time spent in treatment. Examining the impact of length of treatment involvement on program outcomes could inform the placement of noncompleters readmitted to the program. It should be noted that due to the small sample of noncompleters within the current evaluation, creating subgroups of noncompleters based on length of treatment involvement and running related analyses was not feasible.

Noncompleters readmitted to $\mathrm{MDH}$ could form an additional comparison group for exploring time spent in treatment. Readmissions to $\mathrm{MDH}$ were not included in the present analyses because these offenders had, to some extent, already participated in the program. In any future evaluation of $\mathrm{MDH}$, comparisons between readmissions and program completers could be considered. These group comparisons would facilitate the assessment of treatment involvement beyond the standard eight weeks. Analyses would determine whether readmissions who complete the program have better outcomes than 
program completers who go through the program once. These results could provide support for extending the program over the recommended 3- to 9-month period.

The postprogram client survey was based on the MDH client satisfaction questionnaire. As a result, not all of the questions in the survey are relevant to the current evaluation. Any future evaluation of the MDH program could look at the client satisfaction data in the postprogram survey, as a measure of program effectiveness. Furthermore, this data could be used to explore whether client satisfaction is associated with program outcomes. In another evaluation, it may also be of interest to add a couple of questions to the postprogram client survey that ask offenders to provide suggestions for program improvement. This supplementary client feedback may inform program modification.

In the correctional substance abuse treatment literature, there appears to be a growing interest in developing a model for predicting treatment outcomes, as it is felt little is known about the type of substance-abusing offender most apt to succeed in the rehabilitation process (Inciardi et al., 2004; Marlowe, Patapis, \& DeMatteo, 2003). Consistent with this recent interest of the correctional evaluation community, the present evaluation explored potential predictors of program completion. Any future evaluation of the MDH program could continue to develop a prediction model. This type of investigation could not only validate the findings from the current evaluation, but also potentially inform clinical decisions with respect to offenders at high risk of program failure.

Although MDH is a medium to high intensity treatment intended for medium to high risk offenders, there are still a few offenders admitted to the program who are low 
risk. The literature has demonstrated that the best program outcomes are attained when risk level and treatment intensity are matched (Andrews \& Bonta, 2003; Andrews, Zinger et al., 1990; Bonta, 1996; Gendreau, 1996; Farabee et al., 1999; McGuire \& Priestley, 1995). Given that this evaluation did not examine recidivism, it is not known what types of outcomes these low risk offenders had with respect to recidivism in comparison to medium and high risk offenders. Any future evaluation of MDH could assess this unknown.

Future directions for correctional substance abuse programming. In light of the findings from the current evaluation, directions for future research on correctional substance abuse programming can be recommended. Standardized instruments were used in the current evaluation to measure knowledge gain on problem solving and assertiveness. Neither of these instruments specified the total score an offender needed to attain to suggest a meaningful increase in knowledge. Without these guidelines, the present evaluation, as well as past studies, reported personal gain in a dichotomous fashion (i.e., whether knowledge increased or decreased). Due to the limited information on amount of knowledge gain, little is known about how much personal gain is required from a substance abuse program to generate program success. Specifically, it is unclear how much knowledge or ability is considered a sufficient increase to reasonably assume offenders are equipped to change their behaviour. This shortcoming of the literature highlights two areas for future research. First, work should be undertaken to determine whether amount of personal gain can in fact be measured. Second, if it can be measured, research should establish how much gain is needed for successful behaviour change. 
Research in these areas could provide further understanding of how correctional substance abuse interventions work.

Increased confidence or self-efficacy is a program outcome of the relapse prevention treatment approach. This construct appears to be similar to the self-deceptive enhancement profile of the BIDR, which describes individuals with high scores as being overconfident. Interestingly, recent research suggests that socially desirable responding, which encompasses both self-deceptive enhancement and impression management, among offenders may be a reflection of prosocial behaviour (Robertson, 1999). Accordingly, desirable responding among offenders may not be a negative characteristic, but a representation of positive functioning. Given that many cognitive-behavioural substance abuse programs incorporate relapse prevention in its design and measure socially desirable responding, it would be beneficial to have research conducted on the relationship between desirable responding and confidence. Indeed, it would be of value to know whether measures of socially desirable responding, such as the BIDR, are related to measures of confidence, such as the DTCQ. In addition, determining whether the BIDR is in fact measuring prosocial functioning among offenders would be useful. This research would provide additional information on the two constructs, which could be applied to both research and practice.

New ground was broken with the current evaluation as it was designed according to the $\mathrm{MDH}$ program theory. This theory-based design resulted in an evaluation of outcomes directly related to the program's activities. By using this approach, the utility of the evaluation was enhanced in several ways. First, the findings can be used to trace the logic of the program and advance understanding of multimodal treatment programs. 
Second, the results can be used to inform program improvement as well as future areas of evaluation for the program.

Besides being advantageous to $\mathrm{MDH}$, the current evaluation also contributes knowledge to the field of evaluation on correctional community-based substance abuse programs. The examination of short- and long-term outcomes verified that this substance abuse treatment program influenced outcomes other than recidivism and relapse. In addition, analyses suggested possible predictors of program completion. Finally, by basing the evaluation framework on the program's theory, the present evaluation highlighted the benefit of incorporating program theory into the evaluation methodology.

Overall, the present evaluation lends support to the effectiveness of the MDH program in achieving its intended objectives. These positive findings should encourage an array of audiences. First, the staff at MDH, given the selfless and substantial investment they make everyday in the rehabilitation of their clientele. Second, the results are important to MDH's stakeholders, because the achievement of the short- and longterm outcomes can ultimately lead to reintegration success. Finally, the outcomes of the current evaluation are relevant to the offenders who participate in the program, since the evaluation validates their opinion that the program helped them achieve their goal of change. 
Evaluation of a Correctional Substance Abuse Program 201

\section{References}

American Psychiatric Association. (1994). Diagnostic and Statistical Manual of Mental Disorders (4th ed.). Washington, D.C.: American Psychiatric Association.

Andrews, D.A. (1989). Recidivism is predictable and can be influenced: Using risk assessments to reduce recidivism. Forum on Corrections Research, 1(2), 11-18.

Andrews, D.A. (1995). The psychology of criminal conduct and effective correctional treatment. In J. McGuire (Ed.), What works: Reducing re-offending (pp. 35-62). New York: Wiley.

Andrews, D.A., \& Bonta, J. (2003). The psychology of criminal conduct (3rd ed.). Ohio: Anderson Publishing Co.

Andrews, D.A., \& Bonta, J. (1998). The psychology of criminal conduct (2nd ed.). Ohio: Anderson Publishing Co.

Andrews, D.A., Bonta, J., \& Hoge, R.D. (1990). Classification for effective rehabilitation: Rediscovering psychology. Criminal Justice and Behavior, 17(1), $19-52$.

Andrews, D.A., Zinger, I., Hoge, R.D., Bonta, J., Gendreau, P., \& Cullen, F.T. (1990). Does correctional treatment work? A clinically relevant and psychologically informed meta-analysis. Criminology, 28(3), 369-404.

Annis, H., \& Graham, J. (1988). Situational Confidence Questionnaire: User's guide. Toronto: Addiction Research Foundation. 
Annis, H., \& Martin, G. (1985). Drug-Taking Confidence Questionnaire. Toronto: Addiction Research Foundation.

Annis, H., Sklar, S.M., \& Turner, N.E. (1997). Drug-Taking Confidence Questionnaire: User's guide. Toronto: Addiction Research Foundation.

Arif, A., \& Westermeyer, J. (1988). Manual of drug and alcohol abuse: Guidelines for teaching in medical and health institutions. New York: Plenum Medical Book Company.

Bandura, A. (1977). Social learning theory. New Jersey: Prentice-Hall Inc.

Baldwin, S. (1990). Alcohol education and offenders. London: B.T. Batsford Ltd.

Baldwin, S., Heather, N., Lawson, A., Ward, M., Robb, E., Greer, W.C., Gamba, S., \& Robertson, I. (1991a). Comparison of effectiveness: Behavioural and talk-based alcohol education courses for court-referred young offenders. Behavioural Psychotherapy, 19, 157-172.

Baldwin, S., Heather, N., Lawson, A., Ward, M., Robb, E., Greer, W.C., Gamba, S., \& Robertson, I. (1991b). Effectiveness of pre-release alcohol education courses for young offenders in a penal institution. Behavioural Psychotherapy, 19, 321-331.

Beal, C., Weekes, J.R., Millson, W.A., \& Eno, J. (1996). Computerized vs. personal interviews for the identification of offender substance abuse problems. Paper presented at the meeting of the Canadian Psychological Association, Toronto, Ontario. 
Bell, A., \& Rollnick, S. (1996). Motivational interviewing in practice: A structured approach. In F. Rotgers, D.S. Keller, \& J. Morgenstern (Eds.), Treating substance abuse: Theory and technique (pp. 266-285). New York: The Guilford Press.

Boe, R., Nafekh, M., Vuong, B., Sinclair, R., \& Cousineau, C. (2003). The changing profile of the federal inmate population: 1997 and 2002. (Report $\mathrm{N}^{\mathrm{o}} \mathrm{R}-132$ ) Ottawa: Correctional Service of Canada. Report

Boland, F., Henderson, K., \& Baker, J. (1998). Case need domain: Substance abuse assessment review. Forum on Correctional Research, 10(3), 32-34.

Bonta, J. (1996). Risk-needs assessment and treatment. In A.T. Harland, Choosing correctional options that work: Defining the demand and evaluating the supply (pp. 18-32). London: SAGE Publications.

Bonta, J., \& Cormier, R.B. (1999). Corrections research in Canada: Impressive progress and promising prospects. Canadian Journal of Criminology, April 1999, 235-247.

Bonta, J., Law, M., \& Hanson, K. (1998). The prediction of criminal and violent recidivism among mentally disordered offenders: A meta-analysis. Psychological Bulletin, 123(2), 123-142.

Bonta, J., Pang, B., \& Wallace-Capretta, S. (1995). Predictors of recidivism among incarcerated female offenders. The Prison Journal, 75(3), 277-294.

Botvin, G.J. (1990). Substance abuse prevention: Theory, practice, and effectiveness. In M. Tonry \& J.Q Wilson (Eds.), Drugs and crime (pp. 461-519). Chicago: The University of Chicago Press. 
Brochu, S., Cousineau, M., Gillet, M., Cournoyer, L., Pernanen, K., \& Motiuk, L. (2001). Drugs, alcohol, and criminal behaviour: A profile of inmates in Canadian federal institutions. Forum on Corrections Research, 13(3), 30-32.

Brook, R.C., \& Whitehead, P.C. (1980). Drug-free therapeutic community. New York, New York: Human Sciences Press.

Broome, K.M., Knight, K., Hiller M.L., \& Simpson, D.D. (1996). Drug treatment process indicators for probationer and prediction of recidivism. Journal of Substance Abuse Treatment, 13, 487-491.

Brown, J.M., \& Miller, W.R. (1993). Impact of motivational interviewing on participation and outcome in residential alcoholism treatment. Psychology of Addictive Behaviors, 7(4), 211-218.

Burke, B.L., Arkowitz, H., \& Dunn, C. (2002). The efficacy of motivational interviewing and its adaptations: What we know so far. In W.R. Miller \& S. Rollnick (Eds.), Motivational interviewing: Preparing people for change (pp. 217-250). New York: The Guilford Press.

Cahalan, D. (1978). Implications of American drinking practices and attitudes for the prevention and treatment of alcoholism. In G.A. Marlatt \& P.E. Nathan (Eds.), Behavioral approaches to alcoholism (pp. 6-26). New Brunswick, New Jersey: Rutgers Center of Alcohol Studies.

Cahalan, D., \& Cisin, I. (1976). Drinking behavior and drinking problems in the United States. In B. Kissin \& H. Begleiter (Eds.), Social aspects of alcoholism (pp. 77115). New York: Plenum Press. 
Centre on Addiction and Substance Abuse (1998). Behind bars: Substance abuse and America's prison population. New York: Columbia University.

Chan, M., Sorensen, J.L., Guydish, J., Tajima, B., \& Acampora, A. (1997). Client satisfaction with drug abuse day treatment versus residential care. Journal of Drug Issues, 27(2), 367-377.

Chaney, E.F. (1989). Social skills training. In R.K. Hester \& W.R. Miller (Eds.), Handbook of alcoholism treatment approaches (pp. 206-221). New York: Pergamon Press.

Chanhatasilpa, C., MacKenzie, D.L., \& Hickman, L.J. (2000). The effectiveness of community-based programs for chemically dependent offenders: A review and assessment of the research. Journal of Substance Abuse Treatment, 19, 383-393.

Cohen, J. (1992). A power primer. Psychological Bulletin, 112, 155-159.

Conners, N.A., \& Franklin, K.K. (2000). Using focus groups to evaluate client satisfaction in an alcohol and drug treatment program. Journal of Substance Abuse Treatment, 18, 313-320.

Correctional Service of Canada (2003). Standards for correctional programs (726-1). Ottawa: Correctional Service of Canada.

Correctional Service of Canada. (2000). Performance report. Ottawa: Correctional Service of Canada.

Correctional Service of Canada. (1999). Report on plans and priorities 2000-2001. Ottawa: Correctional Service of Canada. 
Correctional Service of Canada (1998a). Corrections in Canada. Ottawa: Career and Management Division.

Correctional Service of Canada (1998b). CSC national substance abuse programs: An application for accreditation. Ottawa: Correctional Service of Canada.

Correctional Service of Canada (1996a). Literature review: Substance abuse treatment modalities. Ottawa: Correctional Research and Development.

Correctional Service of Canada (1996b). Offender substance abuse pre-release program: "Train the trainers" training manual. Ottawa: Correctional Research and Development.

Correctional Service of Canada (1993). “Choices": A community correctional brief treatment relapse prevention and maintenance program. Ottawa: Correctional Service of Canada.

Correctional Service of Canada (1991). Task force on the reduction of substance abuse: Final report. Ottawa: Correctional Service of Canada.

Dagger, A. (2002). Improving program performance. Presentation presented the Essential Skills Series of the Canadian Evaluation Society, Ottawa, ON.

Daly, D.C., Salloum, I.M., Zuckoff, A., Kirisci, L., \& Thase, M.E. (1998). Increasing treatment adherence among outpatients with depression and cocaine dependence: Results of a pilot study. American Journal of Psychiatry, 155(11), 1611-1613.

Delany, P.J., Fletcher, B.W., \& Shields, J.J. (2003). Reorganizing care for the substance using offender: The case for collaboration. Federal Probation, 67(2), 64-68. 
De Leon, G. (1995). Therapeutic communities for addictions: A theoretical framework. International Journal of the Addictions, 30, 1603-1645.

DiClemente, C.C. (2003). Addiction and change: How addictions develop and addicted people recover. New York: The Guilford Press.

DiClemente, C.C, \& Velasquez, M.M. (2002). Motivational interviewing and the Stages of Change. In W.R. Miller \& S. Rollnick (Eds.), Motivational interviewing: Preparing people for change (2nd ed., pp. 201-216). New York: The Guilford Press.

Dimeff, L.A., \& Marlatt, G.A. (1998). Preventing relapse and maintaining change in addictive behaviors. Clinical Psychology: Science and Practice, 5(4), 513-525.

Dobson, K.S., \& Dozois, D.J.A. (2001). Historical and philosophical bases of the cognitive-behavioral therapies. In K.S. Dobson (Ed.), Handbook of cognitivebehavioral therapies (2nd ed., pp. 3-39). New York: The Guilford Press.

Donovan, D.M. (2003). Relapse prevention in substance abuse treatment. In J.L. Sorensen, R.A. Rawson, J. Guydish, \& J.E. Zweben (Eds.), Drug abuse treatment through collaboration: Practice and research partnerships that work. (pp. 121137).

D'Orban, P.T. (1991). The crime connection: alcohol. In I.B. Glass (Ed.), The international handbook of addiction behaviour (pp. 295-300). Routledge: New York. 
Dowden, C. (1998). A meta-analytic examination of the risk, need and responsivity principles and their importance within the rehabilitation debate. Unpublished master's thesis, Psychology Department, Carleton University, Ottawa, Ontario.

Dowden, C., \& Andrews, D.A. (1999). What works for female offenders: A metaanalytic review. Crime and Delinquency, 45(4), 438-452.

Dowden , C., \& Brown, S.L. (2002). The role of substance abuse factors in predicting recidivism: A meta-analysis. Psychology, Crime and Law, 8, 243-264.

D'Zurilla, T.J., \& Nezu, A.M. (1990). Development and preliminary evaluation of the Social Problem-Solving Inventory. Psychological Assessment, 2, 156-163.

Easton, C., Swan, S., \& Sinha, R. (2000). Motivation to change substance use among offenders of domestic violence. Journal of Substance Abuse Treatment, 19, 1-5.

Eisenberg, M. (1999). Three year recidivism tracking of offenders participating in substance abuse treatment programs. Report prepared for the 76 th Texas Legislature, Criminal Justice Policy Council, Austin, Texas.

Eisenberg, M., \& Fabelo, T. (1996). Evaluation of the Texas correctional substance abuse treatment initiative: The impact of policy research. Crime and Delinquency, 42(2), 296-308.

Etheridge, R.M., \& Hubbard, R.L. (2000). Conceptualizing and assessing treatment structure and process in community-based drug dependency treatment programs. Substance Use and Misuse, 35(12-14), 1757-1795. 
Farabee, D., Prendergast, M., Cartier, J., Wexler, H., Knight, K., \& Anglin, M.D. (1999). Barriers to implementing effective correctional drug treatment programs. The Prison Journal, 79(2), 150-162.

Field, G. (1985). The Cornerstone program: A client outcome study. Federal Probation, $49,50-55$.

Gaes, G.G. (2001). Program evaluation: Guidelines for asking the right questions. In L.L Motiuk and R.C. Serin (Eds.), Compendium 2000 on effective correctional programming (pp. 185-189). Ottawa: Correctional Service of Canada.

Gaes, G.G. (1998). Correctional treatment. In M.H. Tonry (Ed.), The handbook of crime and punishment (pp. 712-738). New York, New York: Oxford University Press.

Gendreau, P. (1996). The principles of effective intervention with offenders. In A. T. Harland (Ed.), Choosing correctional options that work: Defining the demand and evaluating the supply (pp. 117-130). London: SAGE Publications.

Gendreau, P., \& Goggin, C. (1997). Correctional treatment: Accomplishments and realities. In P. VanVourhis, M. Braswell, and D. Lester (Eds.), Correctional counselling and rehabilitation (pp. 271-279). Anderson.

Gendreau, P., Goggin, C., \& Little, T. (1996). Predicting adult offender recidivism: What works. Report submitted to the Ministry of the Solicitor General Canada.

Gendreau, P., Goggin, C., \& Smith, P. (2001). Implementation guidelines for correctional programs in the "real" world. In G.A. Bernfeld, D.P. Farrington, \& A.W. Leschied (Eds.), Offender rehabilitation in practice (pp. 247-268). Toronto: John Wiley \& Sons. 
Gendreau, P., Goggin, C., \& Smith, P. (1999). The forgotten issue in effective correctional treatment: Program implementation. International Journal of Offender Therapy and Comparative Criminology, 43(2), 180-187.

Gendreau, P., \& Ross, R.R. (1980). Effective correctional treatment: Biliotherapy for cynics. In R.R. Ross and P. Gendreau (Eds.), Effective correctional treatment. Toronto: Butterworths.

Ginsburg, J.I.D. (2000). Using motivational interviewing to enhance treatment readiness in offenders with symptoms of alcohol dependence. Unpublished doctoral dissertation, Psychology Department, Carleton University, Ottawa, Ontario.

Ginsburg, J.I.D., Mann, R.E., Rotgers, F., \& Weekes, J.R. (2002). Motivational interviewing with criminal justice populations. In W.R. Miller \& S. Rollnick (Eds.), Motivational interviewing: Preparing people for change (pp. 333-345). New York: The Guilford Press.

Glider, P., Mullen, R., Herbst, D., Davis, C., \& Fleishman, B. (1997). Substance abuse treatment in a jail setting: A therapeutic community model. In G. De Leon (Ed.), Community as method: Therapeutic communities for special populations and special settings (pp. 97-112). London: Praeger.

Goss Gilroy Inc. (1999). Substance abuse programs follow-up study: Final report. Report submitted to the Correctional Service of Canada.

Graham, W.F., \& Wexler, H.K. (1997). The Amity Therapeutic Community Program at Donovan prison: Program description and approach. In G. De Leon (Ed.), 
Community as method: Therapeutic communities for special populations and special settings (pp. 97-112). London: Praeger.

Grant, B. (2001). The role and function of addictions research in the Correctional Service of Canada. Forum on Corrections Research, 13(3), 30-32.

Gregoire, T.K., \& Burke, A.C. (2004). The relationship of legal coercion to readiness to change among adults with alcohol and other drug problems. Journal of Substance Abuse Treatment, 26, 35-41.

Harper, R., \& Hardy, S. (2000). An evaluation of motivational interviewing as a method of intervention with clients in a probation setting. British Journal of Social Work, $30,393-400$.

Harris, P., \& Smith, S. (1996). Developing community corrections: An implementation perspective. In A.T. Harland, Choosing correctional options that work: Defining the demand and evaluating the supply (pp. 183-222). London: SAGE Publications.

Hartmann, D.J., Wolk, J.L., Johnston, J.S., \& Colyer, C.J. (1997). Recidivism and substance abuse outcomes in a prison-based therapeutic community. Federal Probation, 61(4), 18-25.

Hawkins, J., Catalano, R., \& Wells, E. (1986). Measuring effects of skills training intervention for drug abusers. Journal of Consulting and Clinical Psychology, $54(5), 661-664$ 
Heather, N., Luce, A., Peck, D., Dunbar, B., \& James, I. (1999). Development of a treatment version of the Readiness to Change Questionnaire. Addiction Research, 7(1), 63-68.

Heather, N., Rollnick, S., Bell, A., \& Richmond, R. (1996). Effects of brief counselling among male heavy drinkers identified on general hospital wards. Drug and Alcohol Review, 15, 29-38.

Hiller, M.L., Knight, K., Devereux, J., \& Hathcoat, M. (1996). Posttreatment outcomes for substance-abusing probationers mandated to residential treatment. Journal of Psychoactive Drugs, 28(3), 291-296.

Hodgins, D.C. \& Lightfoot, L.O. (1989). The use of the Alcohol Dependence Scale with incarcerated male offenders. International Journal of Offender Therapy and Comparative Criminology, 33, 59-67.

Hollin, C.R. (1994). Substance abuse and offending. Current Opinion in Psychiatry, 7, 462-465.

Inciardi, J.A., Martin, S.S., \& Butzin, C.A. (2004). Five-year outcomes of therapeutic community treatment of drug-involved offenders after release from prison. Crime and Delinquency, 50(1), 88-107.

Inciardi, J.A., Martin, S.S., Butzin, C.A., Hooper, R.M., \& Harrison, L.D. (1997). An effective model of prison-based treatment for drug-involved offenders. Journal of Drug Issues, 27(2), 261-278. 
Irvin, J.E., Bowers, C.A., Dunn, M.E., \& Wang, M.C. (1999). Efficacy of relapse prevention: A meta-analytic review. Journal of Consulting and Clinical Psychology, 67, 563-570.

Jamieson, J.D., \& Stone, W.E. (1991). Predicting DWI education success. Federal Probation, 55, 43-47.

Judd, C.M., \& Kenny, D.A. (1981). Process analysis: Estimating mediation in treatment evaluations. Evaluation Review, 5(5), 602-619.

Knight, K., \& Hiller, M.L. (1997). Community-based substance abuse treatment: A 1year outcome evaluation of the Dallas County Judicial Treatment Center. Federal Probation, 61(2), 61-68.

Kroner, D.G., \& Weekes, J.R. (1996). Balanced Inventory of Desirable Responding: Factor structure, reliability, and validity with an offender sample. Personality and Individual Differences, 21(3), 323-333.

Labute, G. (2002). Mathwizz (University of Calgary). Retrieved December 2, 2002, from http://www.mathwizz.com/statistics/help/help4.htm

Langevin, C.M. (1999). A profile of alcohol-abusing offenders. Unpublished masters thesis, Psychology Department, Carleton University, Ottawa, Ontario.

Langevin, C.M., Blanchet, S., \& Long, C. (1999). Strategy for substance abuse treatment. Let's Talk, 24(5), 17-18.

Lightfoot, L.O. (2001). Programming for offenders with substance abuse and dependence problems. In L.L Motiuk and R.C. Serin (Eds.), Compendium 2000 on effective correctional programming (pp. 98-112). Ottawa: Correctional Service of Canada. 
Lightfoot, L.O. (1997). Treating substance abuse and dependence in offenders: A review of methods and outcomes. Paper presented at the ICCA's Research Conference, Cleveland, Ohio.

Lightfoot, L.O., \& Hodgins, D. (1988). A survey of alcohol and drug problems in incarcerated Canadian offenders. International Journal of the Addictions, 23(7), 687-706.

Lipsey, M.W. (1995). What do we learn from 400 research studies on the effectiveness of treatment with juvenile delinquents? In J. McGuire (Ed.), What works: Reducing reoffending - Guidelines from research and practice (pp. 63-78). Toronto: John Wiley \& Sons.

Lipton, D.S. (1998). Therapeutic community treatment programming in corrections. Psychology, Crime and Law, 4, 213-263.

Lipton, D.S. (1995). The effectiveness of treatment for drug abusers under criminal justice supervision. Presentation made at the annual conference on research and evaluation, sponsored by the National Institute of Justice, Washington, D.C.

Lipton, D.S., Falkin, G.P., \& Wexler, H.K. (1992). Correctional drug abuse treatment in the United States: An overview. In C.G. Leukefeld and F.M. Tims (Eds.), Drug abuse treatment in prisons and jails (pp. 8-30). Rockville: National Institute on Drug Abuse.

Lipton, D.S., Thornton, D., McGuire, J., Porporino, F.J., \& Hollin, C.R. (2000). Program accreditation and correctional treatment. Substance Use and Misuse, 35(12-14), $1705-1734$. 
Lockwood, D., Inciardi, J.A., Butzin, C.A., \& Hooper, R.M. (1997). The therapeutic community continuum in corrections. In G. De Leon (Ed.), Community as method: Therapeutic communities for special populations and special settings (pp. 87-96). London: Praeger.

Lösel, F. (2001). Evaluating the effectiveness of correctional programs: Bridging the gap between research and practice. In G.A. Bernfeld, D.P. Farrington, \& A.W. Leschied (Eds.), Offender rehabilitation in practice (pp. 67-92). Toronto: John Wiley \& Sons.

Lösel, F. (1995). The efficacy of correctional treatment: A review and synthesis of metaevaluations. In J. McGuire (Ed.), What works: Reducing reoffending - Guidelines from research and practice (pp. 79-111). Toronto: John Wiley \& Sons.

Marlatt, G.A., \& Gordon, J.R. (1985). Relapse prevention: Maintenance strategies in the treatment of addictive behaviors. New York: Guilford.

Marlatt, G.A., Baer, J.S., Kivlahan, D.R., Dimeff, L.A., Larimer, M.E., Quigley, L.A., Somers, J.M., \& Williams, E. (1998). Screening and brief intervention for highrisk college student drinkers: Results from a 2-year follow-up assessment. Journal of Consulting and Clinical Psychology, 66(4), 604-615.

Marlowe, D.B., Patapis, N.S., \& DeMatteo, D.S. (2003). Amenability to treatment of drug offenders. Federal Probation, 67(2), 40-46.

Marshall, C., \& Rossman, G.B. (1995). Designing qualitative research. London: SAGE Publications. 
Martin, S.S., Butzin, C.A., \& Inciardi, J.A. (1995). Assessment of a multistage therapeutic community for drug-involved offenders. Journal of Psychoactive Drugs, 27(1), 109-116.

Martin, S.S., Butzin, C.A., Saum, C.A., \& Inciardi, J.A. (1999). Three-year outcomes of therapeutic community treatment for drug-involved offenders in Delaware: From prison to work release to aftercare. The Prison Journal, 79(3), 294-320.

Martinson, R. (1974). What works? Questions and answers about prison reform. The Public Interest, 10, 22-54.

McGuire, J., Broomfield, D., Robinson, C., \& Rowson, B. (1995). Short-term effects of probation programs: An evaluation study. International Journal of Offender Therapy and Comparative Criminology, 39(1), 23-42.

McGuire, J., \& Priestley, P. (1995). Reviewing "what works": Past, present and future. In J. McGuire (Ed.), What works: Reducing reoffending - Guidelines from research and practice (pp. 3-34). Toronto: John Wiley \& Sons.

Messina, N., Wish, E., \& Nemes, S. (2001). Therapeutic community treatment may reduce future incarceration: A research note. Federal Probation, 65(3), 40-45.

Messina, N., Wish, E., \& Nemes, S. (1999). Predictors of treatment outcomes in men and women admitted to a therapeutic community. Report submitted to the Centre for Substance Abuse Treatment, US Department of Health and Human Services.

Miller, W.R. (1983). Motivational interviewing with problem drinkers. Behavioural Psychotherapy, 11, 147-172. 
Miller, W.R. (1985). Motivation for treatment: A review with special emphasis on alcoholism. Psychological Bulletin, 98(1), 84-107.

Miller, W.R. (1989). Follow-up assessment. In R.K. Hester \& W.R. Miller (Eds.), Handbook of alcoholism treatment approaches (pp. 81-89). New York: Pergamon Press.

Miller, W.R., \& Hester, R.R. (1986). The effectiveness of alcoholism treatment: What research reveals. In W.R. Miller \& N. Heather (Eds.), Treating addictive behaviours: Processes of change (pp. 121-174). New York: Plenum Press.

Miller, W.R., \& Kurtz, E. (1994). Models of alcoholism used in treatment: Contrasting AA and other perspectives with which it is often confused. Journal of Studies on Alcohol, 55, 159-166.

Miller, W.R., \& Rollnick, S. (2002). Motivational interviewing: Preparing people for change. New York: The Guilford Press.

Millson, W.A., \& Robinson, D. (1992). An assessment of the Offender Substance Abuse Pre-Release Program at Drumheller Institution. (Report $\mathrm{N}^{\mathrm{o}} \mathrm{R}-26$ ). Ottawa: Correctional Service of Canada.

Millson, W.A., Robinson, D., Porporino, F.J., \& Weekes, J.R. (1993). Substance abuse severity and criminal behaviour. Paper presented at the annual meeting of the Canadian Psychological Association, Montréal, Québec.

Millson, W.A., Weekes, J.R., \& Lightfoot, L.O. (1995). The Offender Substance Abuse Pre-Release Program: Analysis of intermediate and post-release outcomes. (Report $\mathrm{N}^{0} \mathrm{R}-40$ ). Ottawa: Correctional Service of Canada. 
Monahan, J. (1996). Violence prediction: The past twenty and the next twenty years. Criminal Justice and Behavior, 23(1), 107-120.

Monahan, J., \& Steadman, H.J. (1994). Toward a rejuvenation of risk assessment research. In J. Monahan \& H.J. Steadman (Eds.), Violence and mental disorder: Developments in risk assessment (pp. 1-17). Chicago: University of Chicago Press.

Montagne, M., \& Scott, D.M. (1993). Prevention of substance abuse problems: Models, factors, and processes. International Journal of the Addictions, 28(12), $1177-$ 1208.

Monti, P.M., Abrams, D.B., Kadden, R.M., \& Cooney, N.T. (1989). Treating alcohol dependence. New York: Guilford Press.

Moos, R. (1975). Evaluation correctional and community settings. New York: John Wiley \& Sons.

Morgan, T.J. (1996). Behavioral treatment techniques for psychoactive substance use disorders. In F. Rotgers, D.S. Keller, \& J. Morgenstern (Eds.), Treating substance abuse: Theory and technique (pp. 174-201). New York: Guilford Press.

Morris, R. (2001). Alcohol and drugs: A perspective from New Zealand. Forum on Corrections Research, 13(3), 18-19.

Motiuk, L., Boe, R., \& Nafekh, M. (2003). Statistical overview - April 2003: The safe return of offenders to the community. Ottawa: Correctional Service of Canada.

Motiuk, L., Boe, R., \& Nafekh, M. (2002). Statistical overview-April 2002: The safe return of offenders to the community. Ottawa: Correctional Service of Canada. 
Mumola, C.J. (1999). Substance abuse and treatment, State and Federal prisoner, 1997. Washington, DC: Bureau of Justice Statistics.

Munro, B.H., \& Page, E.B. (1993). Statistical methods for health care research (2nd ed.). Philadelphia, PE: J.B. Lippincott Company.

Murray, D.W. (1996). Drug abuse treatment in the Federal Bureau of Prisons: A historical review and assessment of contemporary initiatives. In K.E. Early (Ed.), Drug treatment behind bars: Prison-based strategies for change (pp. 89-100). London: Praeger.

Nemes, S., Messina, N., Wish, E., \& Wraight, B. (1999). Opening the black box: The impact of in-patient treatment services on client outcomes. Report submitted to the Centre for Substance Abuse Treatment, US Department of Health and Human Services.

Nielsen, A.L., Scarpitti, F.R., \& Inciardi, J.A. (1996). Integrating the therapeutic community and work release for drug-involved offenders. Journal of Substance Abuse Treatment, 13, 349-358.

Ogborne, A.C. (1997). Theories of "addiction" and implications for counseling. In S. Harrison \& V. Carver (Eds.), Alcohol and drug problems: A practical guide for counselors (2nd ed., pp. 3-18). Toronto: Addiction Research Foundation.

Orbis Partners Inc. (2002). Program and outcome review of Maison Decision House: A community-based substance abuse facility for male federal offenders. A report submitted to the Correctional Service of Canada: Ontario Regional Headquarters. 
Palmer, T. (1995). Programmatic and nonprogrammatic aspects of successful intervention: New directions for research. Crime and Delinquency, 41(1), 100131.

Parks, G.A., Marlatt, G.A., Young, C., \& Johnson, B. (2003). Relapse prevention therapy as a case management tool for high-risk and high-need offenders. Paper presented at the Solicitor General Canada conference, Montréal, Québec.

Paulhus, D.L. (1998). Paulhus Deception Scales (PDS): The Balanced Inventory of Desirable Responding - 7. North Tonawanda, NY: Multi-Health Systems.

Paulhus, D.L. (1991). Measurement and control of response bias: Balanced Inventory of Desirable Responding (BIDR). In J.P. Robinson, P.R. Shaver, \& L.S. Wrightman (Eds.), Measures of personality and social psychological attitudes: Volume I (pp. 37-41). San Diego, CA: Academic Press Inc.

Paulhus, D.L. (1984). Two-component models of socially desirable responding. Journal of Personality and Social Psychology, 46(3), 598-609.

Pearson, F.A., \& Lipton, D.S. (1999). A meta-analytic review of the effectiveness of corrections-based treatments for drug abuse. The Prison Journal, 79(4), 384-410.

Pearson, F.A., Lipton, D.S., Cleland, C.M., \& Yee, D.S. (2002). The effects of behavioral/cognitive-behavioral programs on recidivism. Crime and Delinquency, $48(3), 476-496$

Pedhazur, E.J. (1997). Multiple regression in behavioural research: Explanation and prediction. Forth Worth: Harcourt Brace College Publishers. 
Pelissier, B.M.M., Rhodes, W., Saylor, W., Gaes, G., Camp, S., Vanyur, S.D., \& Wallace, S. (2000). TRIAD drug treatment evaluation project: Final report of three-year outcomes: Part 1. Retrieved March 29, 2002, from http://www.bop.gov/orepg/oretriad.html

Pelissier, B.M.M., Gaes, G., Rhodes, W., Camp, S., O’Neil, J., Wallace, S., \& Saylor, W. (1998). TRIAD drug treatment evaluation project: Six-month interim report. Retrieved July 1999, from http:/www.bop.gov/orepg/oretriad.html

Peters, R.H., Kearns, W.D., Murrin, M.R., Dolente, A.S., \& May, R.L. (1993). Examining the effectiveness of in-jail substance abuse treatment. Journal of Offender Rehabilitation, 19(3/4), 1-39.

Porporino, F.J. (1997). Developing program accreditation criteria for the Correctional Service of Canada: Issues and a suggested approach. A report submitted to the International Panel on Accreditation of Correctional Programs.

Prochaska, J.O., \& DiClemente, C.C. (1986). Toward a comprehensive model of change. In W.R. Miller \& N. Heather (Eds.), Treating addictive behaviors: Processes of change (pp. 3-27). New York: Plenum Press.

Prochaska, J.O., \& DiClemente, C.C. (1992). Stages of change in the modification of problem behaviors. In M. Hersen, R.M. Eisler, \& M. Miller (Eds.), Progress in behavior modification (pp. 184-214). Sycamore, IL: Sycamore Press.

Prochaska, J.O., DiClemente, C.C., \& Norcross, J.C. (1992). In search of how people change: Applications to addictive behaviors. American Psychologist, 47(9), 11021114. 
Ramirez, D.E. (1995). Chapter 9: Samples and sampling distributions (University of Virginia). Retrieved December 2, 2002, from http://www.math.virginia.edu/ der/usem170/Chapter09

Rankin, J.G. (1978). An overview of etiological models and theories of problem drinking and alcoholism. Etiology, 8, 10-16.

Robertson, L.A. (1999). Socially desirable responding in the context of self-report anger measures in a forensic setting. Unpublished doctoral dissertation, Psychology Department, Carleton University, Ottawa, Ontario.

Robinson, D., Fabiano, E., Porporino, F.J., Millson, W.A., \& Graves, G. (1993). A guide to the use of the Computerized Lifestyle Assessment Instrument for substance abuse. Ottawa: Correctional Service of Canada.

Robinson, D., Porporino, F.J., \& Millson, W.A. (1991). Patterns of alcohol and drug use among federal offenders as assessed by the Computerized Lifestyle Screening Instrument. (Report $\mathrm{N}^{\circ} \mathrm{R}-11$ ) Ottawa: Correctional Service of Canada.

Rollnick, S., Heather, N., Gold, R., \& Hall, W. (1992). Development of a short "Readiness to Change Questionnaire" for use in brief opportunistic intervention among excessive drinkers. British Journal of Addiction, 87, 734-754.

Ross, R.R., \& Lightfoot, L.O. (1985). Treatment of the alcohol-abusing offender. Springfield, IL: Charles C. Thomas.

Rossi, J.J., \& Filstead, W.J. (1973). The therapeutic community. New York, New York: Behavioral Publications. 
Rossi, P.H., Freeman, H.E., \& Lipsey, M.W. (1999). Evaluation: A systematic approach (6th ed.). Thousand Oaks, CA: SAGE Publications.

Rotgers, F. (1996). Behavioral theory of substance abuse treatment: Bringing science to bear on practice. In F. Rotgers, D.S. Keller, \& J. Morgenstern (Eds.), Treating substance abuse: Theory and technique (pp. 174-201). New York: Guilford Press.

Roy, M. (2001). The national drug strategy for the Correctional Service of Canada. Forum on Corrections Research, 13(3), 5-6.

Runyon, R.P., \& Haber, A. (1991). Fundamentals of behavioural statistics (7th ed.). Toronto, ON: McGraw-Hill, Inc.

Saunders, B., Wilkinson, C., \& Phillips, M. (1995). The impact of a brief motivational intervention with opiate users attending a methadone programme. Addiction, 90, $415-424$

Saunders, B., Wilkinson, C., \& Towers, T. (1996). Motivation and addictive behaviors: Theoretical perspectives. In F. Rotgers, D.S. Keller. \& J. Morgenstern (Eds.), Treating substance abuse: Theory and technique (pp. 241-265). New York: The Guilford Press.

Scarpitti, F.R., Inciardi, J.A., \& Pottieger, A.E. (1993). Process evaluation techniques for corrections-based drug treatment programs. Journal of Offender Rehabilitation, 19(3/4), 71-79.

Scoles, P., \& Fine, E.W. (1977). Short-term effects of an educational program for drinking drivers. Journal of Studies on Alcohol, 38, 633-637. 
Sellman, J.D., Sullivan, P.F., Dore, G.M., Adamson, S.J., \& MacEwan, I. (2001). A randomized controlled trial of motivational enhancement therapy (MET) for mild to moderate alcohol dependence. Journal of Studies on Alcohol, 62, 389-396.

Serin, R.C. (2001). Program evaluation: Intermediate measures of treatment success. In L.L Motiuk \& R.C. Serin (Eds.), Compendium 2000 on effective correctional programming (pp. 98-112). Ottawa: Correctional Service of Canada.

Serin, R.C., \& Kennedy, S. (1997). Treatment readiness and responsivity: Contributing to effective correctional programming. Ottawa: Correctional Service of Canada.

Sia, T.L., Dansereau, D.F., \& Czuchry, M.L. (2000). Treatment readiness training and probationers' evaluation of substance abuse treatment in a criminal justice setting. Journal of Substance Abuse Treatment, 19, 459-467.

Siegal, H.A., Wang, J., Carlson, R.G., Falck, R.S., Rahman, A.M., \& Fine, R.L. (1999). Ohio's prison-based therapeutic community treatment programs for substanceabusers: Preliminary analysis of re-arrest data. Journal of Offender Rehabilitation, $28(3 / 4), 33-48$.

Skinner, H.A. (1982). The Drug Abuse Screening Test. Toronto: Addiction Research Foundation.

Skinner, H.A., \& Horn, J.L. (1984). Alcohol Dependence Scale: Users' guide. Toronto: Addiction Research Foundation.

Sobell, M.B., \& Sobell, L.C. (1993). Problem drinkers - Guided self-change treatment. New York: Guilford Press. 
Soden, T., \& Murray, R. (1997). Motivational interviewing techniques. In S. Harrison \& V. Carver (Eds.), Alcohol and drug problems: A practical guide for counselors (2nd ed., pp. 3-18). Toronto: Addiction Research Foundation.

Staton, M., Leukefeld, C., Logan, T.K., \& Purvis, R. (2000). Process evaluation for a prison-based substance abuse program. Journal of Offender Rehabilitation, $32(1 / 2), 105-127$.

Stephens, R.S., Roffman, R.A., \& Curtin, L. (2000). Comparison of extended versus brief treatments for marijuana use. Journal of Consulting and Clinical Psychology, $68(5), 898-908$.

Stevens, J.P. (2002). Applied multivariate statistics for the social sciences (4th ed.). New Jersey: Lawrence Erlbaum Associates, Publishers.

Swartz, J.A., Lurigio, A.J., \& Slomka, S.A. (1996). The impact of IMPACT: An assessment of the effectiveness of a jail-based treatment program. Crime and Delinquency, 42(4), 553-573.

Swenson. P.R., \& Clay, T.R. (1980). Effects of short-term rehabilitation on alcohol consumption and drinking-related behaviours: An eight month follow-up study of drunk drivers. International Journal of the Addictions, 15, 821-838.

$\mathrm{T}^{3}$ Associates Inc. (1999a). An outcome evaluation of prison-based substance abuse programming: OSAPP, CHOICES and ALTO-Executive Summary. Ottawa: Correctional Service of Canada. 
$\mathrm{T}^{3}$ Associates Inc. (1999b). An outcome evaluation of CSC substance abuse programs: OSAPP, CHOICES and ALTO - Final Report. Ottawa: Correctional Service of Canada.

$\mathrm{T}^{3}$ Associates Inc. (1999c). An outcome evaluation of CSC substance abuse programs: OSAPP, CHOICES and ALTO - Volume III. Ottawa: Correctional Service of Canada.

Taylor, G. (1998). Offender needs: Providing the focus for our correctional interventions. Forum on Corrections Research, 10(3), 3-8.

Tonry, M. (1990). Research on drugs and crime. In M. Tonry \& J.Q Wilson (Eds.), Drugs and crime (pp. 1-8). Chicago: The University of Chicago Press.

Treasury Board Secretariat (2001). Guide for the development of results-based management and accountability frameworks. Ottawa: Treasury Board Secretariat.

Tutty, L.M., Rothery, M.A., \& Grinnell, R.A. (1996). Qualitative research for social workers. Toronto, Ontario: Allyn and Bacon.

US Department of Justice. (1998a). Profile of jail inmates. Washington, DC: US Department of Justice.

US Department of Justice. (1998b). Substance abuse and treatment of adults on probation. Washington, DC: US Department of Justice.

US Department of Justice. (1997). Characteristics of adults on probation. Washington, DC: US Department of Justice.

US Department of Justice. (1995). The effectiveness of treatment for drug abusers under criminal justice supervision. Washington, DC: Office of Justice Programs. 
Vanderburg, S.A., (2002). Motivational interviewing as a precursor to a substance abuse program for offenders. Unpublished doctoral dissertation, Psychology Department, Carleton University, Ottawa, Ontario.

Vigdal. G.L., Stadler, D.W., Goodrick, D.D., \& Sutton, D.J. (1980). Skills training in a program for problem-drinking offenders: A one-year follow-up evaluation. Journal of Offender Counseling, Services and Rehabilitation, 5(2), 61-73.

Weekes, J.R., Fabiano, E., Porporino, F.J., Robinson, D., \& Millson, W.A. (1993). Assessment of substance abuse in offenders: The Computerized Lifestyle Assessment Inventory. Paper presented at the annual meeting of the Canadian Psychological Association, Montréal, Québec.

Weekes, J.R., Moser, A.E., \& Langevin, C.M. (1997). Assessing substance-abusing offenders for treatment. Ottawa: Substance Abuse Program Unit, Correctional Programs Branch, Correctional Service of Canada.

Wexler, H.K. (1994). Progress in prison substance abuse treatment: A five year report. Journal of Drug Issues, 24(2), 349-360.

Wexler, H.K. (1995). The success of therapeutic communities for substance abusers in American prisons. Journal of Psychoactive Drugs, 27(1), 57-66.

Wexler, H.K., Blackmore, J., \& Lipton, D.S. (1991). Project Reform: Developing a drug abuse treatment strategy for corrections. The Journal of Drug Issues, 21(2), 469490. 
Wexler, H.K., DeLeon, G., Thomas, G., Kressel, D., \& Peters, J. (1999). The Amity Prison TC evaluation: Reincarceration outcomes. Criminal Justice and Behavior, 26(2), 147-167.

Wexler, H.K., Falkin, G.P., Lipton, D.S., \& Rosenblum, A.B. (1992). Outcome evaluation of a prison therapeutic community for substance abuse treatment. In C.G. Leukefeld and F.M. Tims (Eds.), Drug abuse treatment in prisons and jails (pp. 156-174). Rockville: National Institute on Drug Abuse.

Wexler, H.K., Melnick, G., Lowe, L., \& Peters, J. (1999). Three-year reincarceration outcomes for Amity in-prison therapeutic community and aftercare in California. The Prison Journal, 79(3), 321-336.

Wexler, H.K., Williams, R.A., Early, K.E., \& Trotman, C.D. (1996). Prison treatment for substance abusers: Stay'N Out revisited. In K.E. Early (Ed.), Drug treatment behind bars: Prison-based strategies for change (pp. 89-100). London: Praeger.

Wong-Reiger, D., \& David, L. (1995). Using program logic to plan and evaluate education and prevention programs. CES Evaluation Methods Sourcebook II, $120-136$.

World Health Organization. (1980). Problems related to alcohol consumption. Geneva.

Yablonsky, L. (1989). The Therapeutic Community: A successful approach for treating substance abusers. New York: Gardner Press.

Zweben, A., \& Zuckoff, A. (2002). Motivational interviewing and treatment adherence. In W.R. Miller \& S. Rollnick (Eds.), Motivational interviewing: Preparing people for change (2nd ed., pp. 299-319). New York: The Guilford Press. 
Evaluation of a Correctional Substance Abuse Program 229

Appendix A

Logic Model: MDH Substance Abuse Treatment Program 


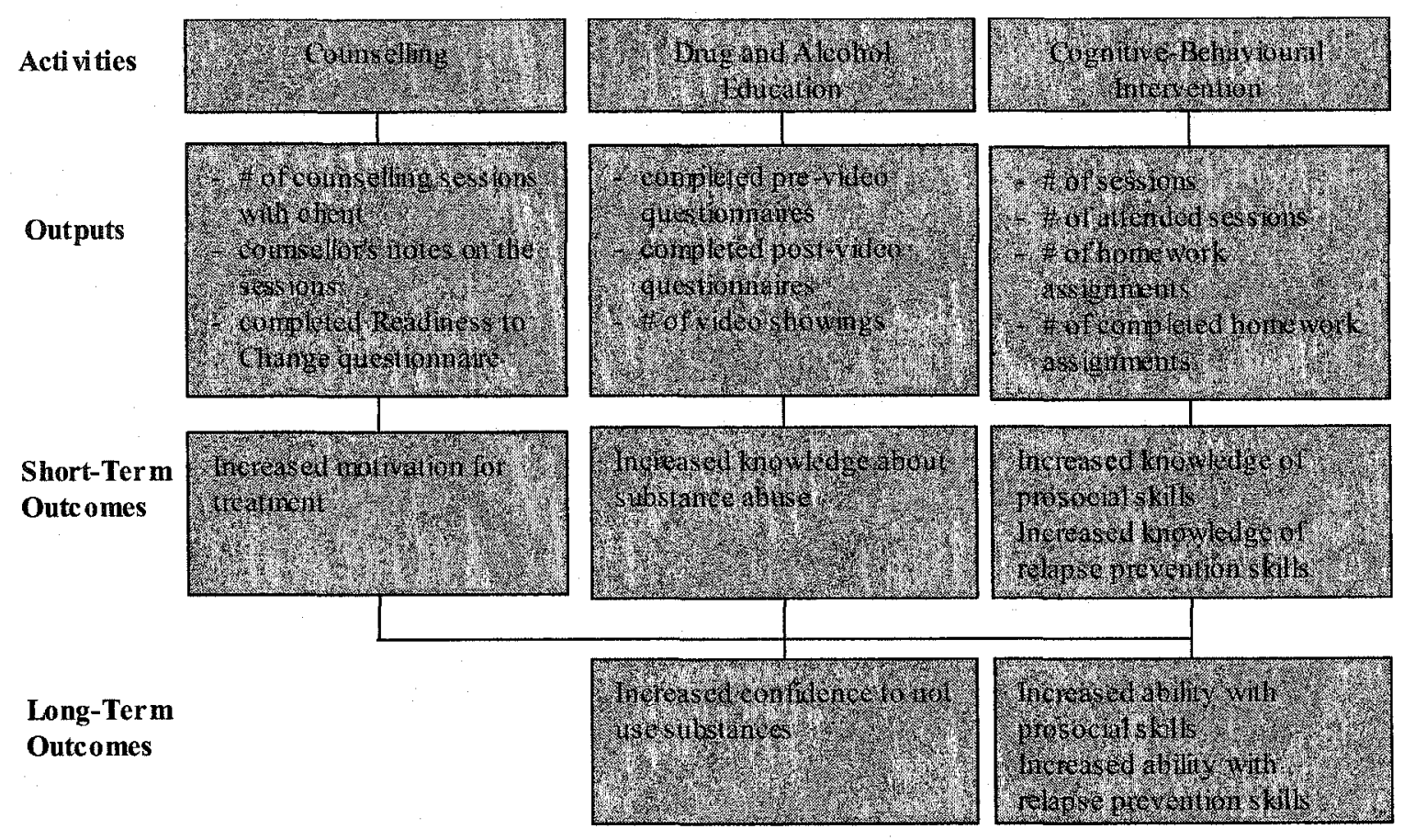


Evaluation of a Correctional Substance Abuse Program 231

Appendix B

Original Logic Model: MDH Substance Abuse Treatment Program 


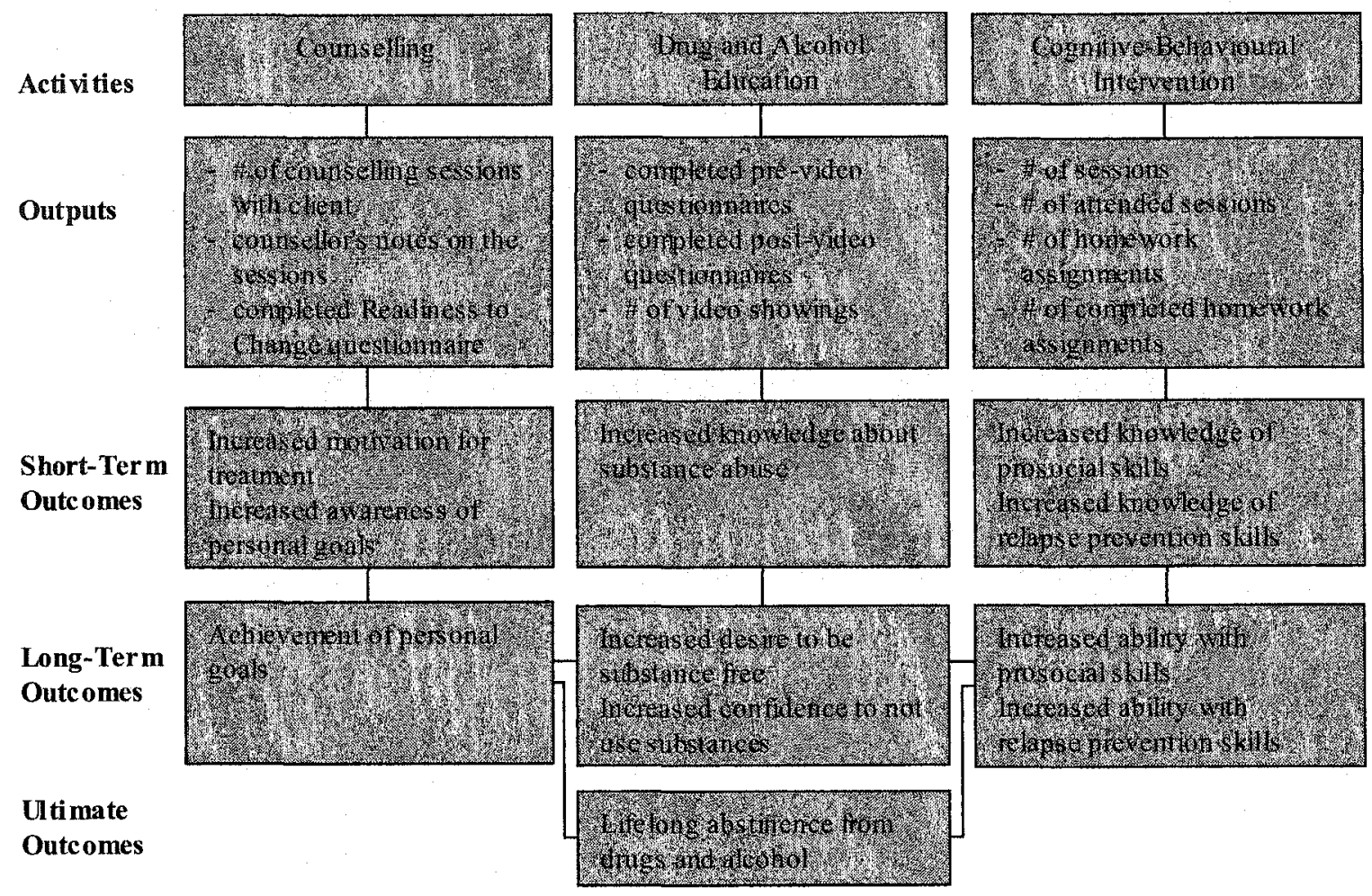


Evaluation of a Correctional Substance Abuse Program 233

Appendix C

Drug-Taking Confidence Questionnaire 


\section{DTCQ}

Listed below are a number of situations or events in which some people experience a drinking problem.

Imagine yourself as you are right now in each of these situations. Indicate on the scale provided how confident you are that you would be able to resist the urge to drink heavily in that situation.

Circle 100 if you are $100 \%$ confident right now that you could resist the urge to drink heavily; 80 if you are $80 \%$ confident; 60 ifs you are $60 \%$ confident. If you are more unconfident than confident, circle 40 to indicate that you are only $40 \%$ confident that you could resist the urge to drink heavily; 20 for $20 \%$ confident; 0 if you have no confidence at all about that situation.

\section{I would be able to resist the urge to drink (use drugs) heavily}

not at all

confident very

confident

1. If I were depressed about things in

020

40

$60 \quad 80$

100 general

2. If I felt shaky, sick or nauseous

$0 \quad 20$

40

$60 \quad 80$

100

3. If I were happy

$0 \quad 20$

40

$60 \quad 80$

100

4. If I felt there was nowhere left to turn

$0 \quad 20$

40

$60 \quad 80$

100

5. If I wanted to see whether I could

$0 \quad 20$

40

$60 \quad 80$

100

drink(use drugs) in moderation

6. If I were in a place where I had used or bought alcohol (drugs) before

7. If I felt tense or uneasy in the presence of $\begin{array}{lllllll}0 & 20 & 40 & 60 & 80 & 100\end{array}$ someone

8. If I were invited to someone's home and

$0 \quad 20 \quad 40$

60

80

100 felt awkward about refusing when they offered me a drink (drugs)

9. If I met some old friends and we wanted to have a good time 
10. If I were unable to express my feelings

$0 \quad 20$

$20 \quad 40$

$40 \quad 60 \quad 80$

100 to someone

11. If I felt that I had let myself down

$\begin{array}{llllll}0 & 20 & 40 & 60 & 80 & 100\end{array}$

12. If I had trouble sleeping

$\begin{array}{llllll}0 & 20 & 40 & 60 & 80 & 100\end{array}$

13. If I felt confident and relaxed

$\begin{array}{llllll}0 & 20 & 40 & 60 & 80 & 100\end{array}$

14. If I were bored

$\begin{array}{llllll}0 & 20 & 40 & 60 & 80 & 100\end{array}$

15. If I wanted to prove to myself that

$0 \quad 20$ alcohol (drugs) was not a problem for me

16. If I unexpectedly found some booze

$0 \quad 20 \quad 40$

60

80

100 (drugs) or happened to see something that reminded me of drinking (doing drugs)

17. If other people rejected me or didn't seem to like me

18. If I were out with friends and they kept suggesting we go somewhere and drink (do drugs)

19. If I were with an intimate friend and we wanted to feel even closer

20. If other people treated me unfairly or interfered with my plans

$\begin{array}{llllll}0 & 20 & 40 & 60 & 80 & 100\end{array}$

$\begin{array}{llllll}0 & 20 & 40 & 60 & 80 & 100\end{array}$

21. If I were lonely

$\begin{array}{llllll}0 & 20 & 40 & 60 & 80 & 100\end{array}$

22. If I wanted to stay awake, be more alert, $\begin{array}{lllllll}0 & 20 & 40 & 60 & 80 & 100\end{array}$ or be more energetic

23. If I felt excited about something $\begin{array}{llllll}0 & 20 & 40 & 60 & 80 & 100\end{array}$

24. If I felt anxious or tense about something $\begin{array}{lllllll}0 & 20 & 40 & 60 & 80 & 100\end{array}$

25. If I wanted to find out whether I could $\quad \begin{array}{lllllll}0 & 20 & 40 & 60 & 80 & 100\end{array}$ take a drink (drugs) occasionally without getting hooked 
not at all

very

confident

confident

26. If I had been using drugs (drinking) and $\begin{array}{lllllll}0 & 20 & 40 & 60 & 80 & 100\end{array}$

thought about drinking (drugs)

27. If I felt that my family was putting a lot $\quad \begin{array}{lllllll}0 & 20 & 40 & 60 & 80 & 100\end{array}$ of pressure on me or that I couldn't measure up to their expectations

28. If others in the same room were drinking $\begin{array}{lllllll}0 & 20 & 40 & 60 & 80 & 100\end{array}$ (doing drugs) and I felt that they expected me to join in

29. If I were with friends and just wanted to $\begin{array}{lllllll}0 & 20 & 40 & 60 & 80 & 100\end{array}$ increase my enjoyment

30. If I were not getting along well with $\quad \begin{array}{lllllll}0 & 20 & 40 & 60 & 80 & 100\end{array}$ others at school or at work

31. If I started to feel guilty about something $\begin{array}{lllllll}0 & 20 & 40 & 60 & 80 & 100\end{array}$

32. If I wanted to lose weight $\quad \begin{array}{lllllll}0 & 20 & 40 & 60 & 80 & 100\end{array}$

33. If I were feeling content with my life $\quad \begin{array}{lllllll}0 & 20 & 40 & 60 & 80 & 100\end{array}$

34. If I felt overwhelmed and wanted to $\quad \begin{array}{lllllll}0 & 20 & 40 & 60 & 80 & 100\end{array}$ escape

35. If I wanted to test out whether I could be $\begin{array}{lllllll}0 & 20 & 40 & 60 & 80 & 100\end{array}$ with friends who drank (do drugs)

without drinking (doing drugs)

36. If I heard someone talking about their $\begin{array}{lllllll}0 & 20 & 40 & 60 & 80 & 100\end{array}$ past drinking (drugs) experiences

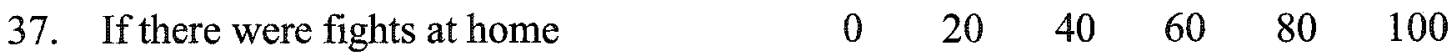

38. If I were pressured to drink (do drugs) $\quad \begin{array}{lllllll}0 & 20 & 40 & 60 & 80 & 100\end{array}$ and felt that I couldn't refuse

39. If I wanted to celebrate with a friend $\quad \begin{array}{llllll}0 & 20 & 40 & 60 & 80 & 100\end{array}$

40. If someone was dissatisfied with my $\quad \begin{array}{lllllll}0 & 20 & 40 & 60 & 80 & 100\end{array}$ work or I felt pressured at school or on the job 
not at all

confident

very
confident

41. If I were angry at the way things had

$\begin{array}{llllll}0 & 20 & 40 & 60 & 80 & 100\end{array}$ turned out

42. If I had a headache or was in physical pain

43. If I remembered something good that had happened

44. If I felt confused about what I should do

$\begin{array}{llllll}0 & 20 & 40 & 60 & 80 & 100\end{array}$

45. If I wanted to test out whether I could be
in places where people were drinking

$\begin{array}{llllll}0 & 20 & 40 & 60 & 80 & 100\end{array}$ (doing drugs) without having a drink (drugs)

46. If I began to think how good having a few drinks (doing drugs) or becoming intoxicated felt

47. If I felt that I needed courage to face up to someone

48. If I were with a group of people and everyone was drinking (doing drugs)

$\begin{array}{llllll}0 & 20 & 40 & 60 & 80 & 100\end{array}$

$0-20-40-60-80$

100 everyone was drinking (doing drugs)

49. If I were having a good time and wanted to increase my sexual enjoyment

50. If I felt that someone was trying to $\begin{array}{llllll}0 & 20 & 40 & 60 & 80 & 100\end{array}$ control me and I wanted to feel more independent 
Evaluation of a Correctional Substance Abuse Program 238

\section{Appendix D}

Readiness to Change Questionnaire - Treatment Version (Alcohol Use) 


\section{RCQ-TV-AU}

Name: FPS \#:

Date Completed:

The following questionnaire is designed to identify how you personally feel about your drinking right now. Please think about your current situation and drinking habits, even if you have given up drinking completely. Read each question below carefully, and then decide whether you agree or disagree with the statements. Please tick the answer of your choice to each question. If you have any questions, please ask the questionnaire administrator.

Your answers are completely private and confidential.

Key:

SD

Strongly

Disagree
D

Disagree

SD D U
$\mathbf{U}$

Unsure drinking because I do not have a problem.

2. I enjoy my drinking but sometimes I drink too much.

3. I am trying to stop drinking or drink less than I used to.

4. There is nothing seriously wrong with my drinking.

5. Sometimes I think I should quit or cut down on my drinking.

6. Anyone can talk about wanting to do something about their drinking, but I am actually doing something about it.
A

Agree

A SA
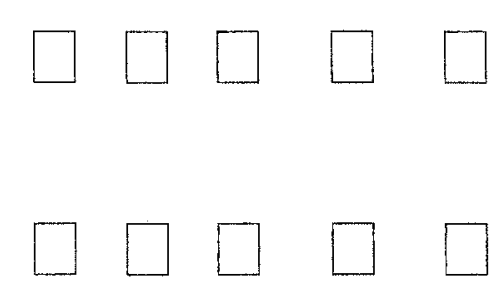

C

For

office use

PC

SA

Strongly

Agree

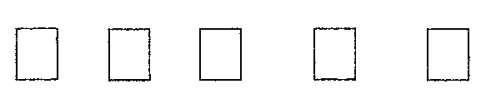

A

PC

$\mathrm{C}$

A 
Key:

SD

\section{Strongly}

Disagree
D

Disagree

SD

Unsure
A

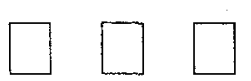

Agree
SA

Strongly

Agree

A SA For

office use

PC

C

A

PC

C

A

PC

14. Sometimes I wonder if my drinking is out of control.

15. I am actively working on my drinking problem.

\section{For office use only}

Please enter the subject's scores below:

PC Score

C Score

A Score 
Evaluation of a Correctional Substance Abuse Program 241

Appendix E

Readiness to Change Questionnaire - Treatment Version (Drug Use) 


\section{$\underline{\text { RCQ-TV-DU }}$}

Name: FPS \#:

Date Completed:

The following questionnaire is designed to identify how you personally feel about your drug use right now. Please think about your current situation and drug using habits, even if you have given up using completely. Read each question below carefully, and then decide whether you agree or disagree with the statements. Please tick the answer of your choice to each question. If you have any questions, please ask the questionnaire administrator.

Your answers are completely private and confidential.

Key:

SD

Strongly

Disagree
$\mathbf{D}$

Disagree

Unsure
A

Agree

SD
D U A SA
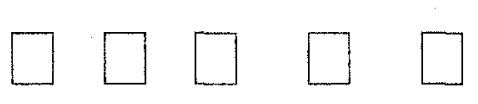

my drug use because I do not have a problem.

2. I enjoy my drug use but sometimes I use too much.

3. I am trying to stop using drugs or use less than I used to.

4. There is nothing seriously wrong with my drug use.

5. Sometimes I think that I should quit or cut down on my drug use.

6. Anyone can talk about wanting to do something about their drug use, but I am actually doing something about it.

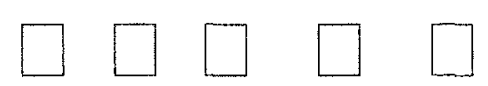

C

A

PC

$\mathrm{C}$

A 
Key:

SD

Strongly

Disagree
D

Disagree

$\mathrm{U}$

Unsure

$\mathrm{SD}$

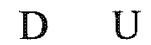

$\square$

7. I am a fairly normal drug user.

8. My drug use is a problem sometimes.
A

Agree

SA

Strongly

Agree

For office

use

PC

C

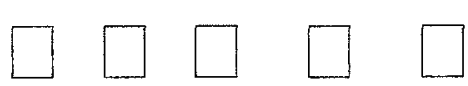

A

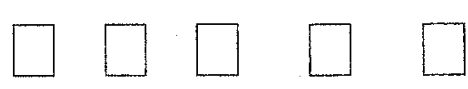

PC

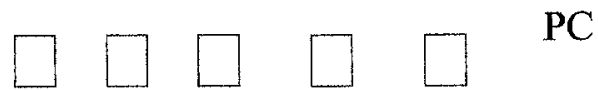

C

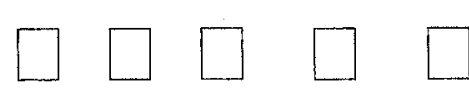
disadvantages of my present drug using habits.

12. I have started to carry out a plan to cut down or quit using drugs.

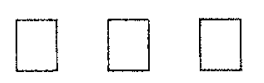

A

PC change about my drug use.
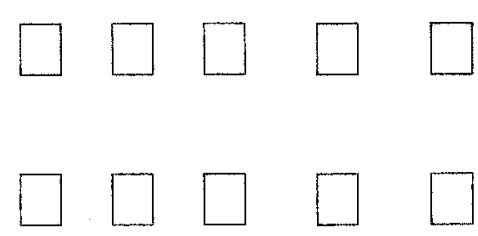

C is out of control.

15. I am actively working on my drug use problem.

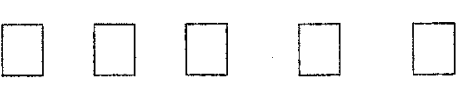

\section{For office use only}

Please enter the subject's scores below:

PC Score

C Score

A Score 
Evaluation of a Correctional Substance Abuse Program 244

Appendix F

Problem-Solving Questionnaire 


\section{PSQ}

Below are a series of statements that describe the way some people might think, feel and behave when they are faced with problems in everyday life. We are talking about important problems that could have a significant effect on your well-being or the well-being of your loved ones, such as health related problems, a dispute with a family member, or a problem with your performance at work or in school. Please read each statement carefully and select one of the numbers below which indicates the extent to which the statement is true of you. Consider yourself as you typically think, feel and behave when you are faced with problems in living these days and place the appropriate number in the parentheses () next to the number of the statement.

$0=$ Not at all true of me

$\mathrm{l}=$ Slightly true of me

2 = Moderately true of me

\section{$3=$ Very true of me \\ $4=$ Extremely true of me}

1. ( ) When I have a problem to solve, one of the things I do is analyze the situation and try to identify what obstacles are keeping me from getting what I want.

2. ( ) Before I try to think of a solution to a problem, I usually set a specific goal that makes clear exactly what I want to accomplish.

3. ( ) When I have a problem to solve, one of the things I do is examine what sort of external circumstances in my environment might be contributing to the problem.

4. ( ) When I am having trouble understanding a problem, I usually try to get more specific and concrete information about the problem to help clarify it.

5. ( ) When I am trying to find a solution to a problem, I often think of a number of possible solutions and then try to combine different solutions to make a better solution.

6. ( ) When I am attempting to solve a problem, I often try to be more creative and think of original or unconventional solutions.

7. ( ) When I am attempting to decide what is the best solution to a problem, I usually try to weigh the consequences of each solution alternative and compare them against each other.

8. ( ) When I am attempting to find a solution to a problem, I try to keep in mind what my goal is at all times.

9. ( ) When I am attempting to find a solution to a problem, I try to approach the problem from as many different angles as possible. 
10. ( ) When I am attempting to decide what is the best solution to a problem, I try to predict the overall outcome of carrying each alternative course of action.

11. ( ) When making decisions, I usually consider not only the immediate consequences of each alternative course of action, but also the long-term consequences.

12. ( ) When I am attempting to decide what is the best solution to a problem, I usually consider the effect that each alternative course of action is likely to have on my personal feelings.

13. ( ) When making decisions, I generally use a systematic method for judging and comparing alternatives.

14. ( ) After carrying out a solution to a problem, I usually try to analyze what went right and what went wrong.

15. ( ) After carrying out a solution to a problem, I usually examine my feelings and evaluate how much they have changed for the better.

16. ( ) Before carrying out a solution to a problem in the actual problematic situation, I often rehearse the solution in order to increase my chances of success.

17. ( ) When the outcome of my solution to a problem is not satisfactory, I usually try to find out what went wrong and then I try again.

18. ( ) After carrying out a solution to a problem, I usually try to evaluate as carefully as possible how much the situation has changed for the better.

19. ( ) When I have a problem to solve, one of the first things I do is get as many facts about the problem as possible.

20. ( ) When I am attempting to solve a problem, I usually think of as many alternative solutions possible until I cannot come up with any more ideas. 
Evaluation of a Correctional Substance Abuse Program 247

Appendix G

Assertiveness Questionnaire 
This test presents descriptions of people who feel uncomfortable because others are drinking, taking drugs, or offering them alcohol or drugs. Each person wants to deal with the problem by using assertiveness techniques. People who use assertiveness techniques are aware of their rights and privileges and use time constructively. Read each item, then circle the letter of the action which describes an assertive response to the situation described. If there is no assertive response presented, circle choice D, "None of the above".

1. Carol is watching television with her husband, and he is drinking. Carol has been trying to reduce the amount she drinks, but the sight of her husband's drink is weakening her willpower. To act assertively, Carol should:

A Concentrate on the television show and try to ignore her urge to drink.

B Ask her husband if he would watch TV in another room when he drinks.

C Move to another room where she can't see his drink.

D None of the above.

2. George and Susan are spending an evening together. After dinner, Susan suggests that they relax in front of the TV with after-dinner drinks. To act assertively, George should:

A Tell Susan that he doesn't want to drink.

B Tell Susan that he has a better idea and ask her to go for a walk.

C Hold a drink without actually drinking it.

D None of the above.

3. When Alan decided to stop using drugs, he got rid of all the drugs in his apartment. His girlfriend Paula still uses, and she has drugs available at her apartment. To act assertively Alan should:
A Ask Paula if she would keep all of her drugs where he won't see it.
B Insist that Paula get rid of all the drugs in her apartment.
C Ask Paula to use only the types of drugs that he does not particularly enjoy.
D None of the above.

4. While at a party, Mark is talking with Rex, an old friend. Rex knows that Mark is trying to stop using drugs, so he decides to offer Mark a joint to test his willpower. To act assertively Mark should:
A Tell Rex that he has much less willpower than he does.
B Accept the joint, but fake inhaling it.
C Inform Rex that he no longer uses.
D None of the above. 
5. Jim has just moved into a new neighbourhood. He wants to be accepted by the other young people there. They tell Jim that he will have to drink a bottle of wine without stopping before he can be part of their crowd. To act assertively, Jim should:

A Keep the crowd entertained with stories about his last neighbourhood.

B Drink the bottle of wine so that he can make new friends.

C Tell the crowd that he does not drink because he doesn't like it.

D None of the above.

6. Dave and his brother are home alone on an evening when their parents are out. When some friends stop by, his brother suggests they drink some of his father's liquor and encourages Dave to join them. To act assertively, Dave should:

A Go to another room and hope that the friends leave before his parents come home.

B Tell his brother that he doesn't want to drink with them.

C Take a drink but make sure that it contains plenty of water.

D None of the above.

7. Mike, who doesn't drink alcohol, pretended to have a drink for his role in a school play. Afterwards, his girlfriend Jane tells him that drinking made him look more mature. She asks him to drink with her at a party. To act assertively Mike should:

A Tell Jane that he doesn't want to drink in order to create an image.

B Accept a drink at the party but not actually drink it.

C Pretend that he didn't hear her and ask Jane if she would like to dance.

D None of the above.

8. Michelle likes to go to baseball games with a group of people from her office. One of her coworkers, Charlie, drinks beer at the games and tries to get Michelle to join him even though he knows she quit drinking. To act assertively Michelle should:

A Make a point of announcing that she stopped drinking a long time ago.

B Ask Charlie not to encourage her to drink because she doesn't drink anymore.

C Start going to baseball games with another group of friends.

D None of the above.

9. Lisa does not drink liquor because she knows she has a difficult time controlling the amount she drinks. Her neighbour Marcy offers Lisa a drink whenever she visits. To act assertively, Lisa should:

A Tell Marcy that she doesn't drink alcohol.

B Tell Marcy that she's not thirsty.

C Say nothing in the interest of keeping their friendship.

D None of the above. 
Evaluation of a Correctional Substance Abuse Program 250

Appendix $\mathrm{H}$

Shame Video Questionnaire 


\section{Shame}

This questionnaire consists of 10 statements about shame and addiction. Some of the statements are true and others are false. If you think a statement is true, circle the word "True". If you think a statement is false, circle the word "False". If you do not know whether a statement is true or false, circle "Don't Know".

1. Guilt is something people feel when they have True False Don't made a mistake or they did something bad.

Know

2. Shame is something people feel when they make several mistakes or they do bad things

True False Don't often.

3. People who feel shame will say things like "I made a mistake" or "I am a bad person".

True False Don't

Know

4. Shame can be a healthy emotion.

True False Don't

Know

5. A person needs to deal with his or her shame to

True False

Don't be able to deal with his or her substance abuse problem.

Know

6. There is no connection between shame and substance-abusing behaviour.

True False Don't

Know

7. Addiction can be described as a dependence on experiences that both change your mood and negatively affect your life.

True False

Don't

Know

8. The term "acting shameless" is used when

True False

Don't referring to someone who does not feel shame.

Know

9. Self-disclosure and socialization can heal shame.

True False

Don't

Know

10. Shame can lead to distorted thinking.

True False Don't

Know 
Evaluation of a Correctional Substance Abuse Program 252

Appendix I

Anger Video Questionnaire 


\section{Anger}

This questionnaire consists of 10 statements about anger. Some of the statements are true and others are false. If you think a statement is true, circle the word "True". If you think a statement is false, circle the word "False". If you do not know whether a statement is true or false, circle "Don't Know".

1. Anger is important because if anger is not dealt True False Don't with it can lead to relapse.

Know

2. Unresolved anger can only come from experiences that occurred in your childhood.

True False Don't

Know

3. Anger can be dealt with by forgiving others. True False Don't

Know

4. Unresolved anger is an emotional response to

True False

Don't perceived injustice or what someone thinks is unfair.

Know

5. The cycle of anger consists of perceived injustice (what someone thinks is unfair), punishment and justifying inappropriate behaviour.

True False

Don't

Know

6. A part of the anger cycle involves a person justifying the inappropriate behaviour of other people.

7. A good way to deal with anger is to not think about it.

True False Don't

Know

8. Anger can be expressed through behaviours, such as screaming, depression and/or substance abuse.

True False

Don't

Know

9. Anger is only caused by other people's actions or behaviour.

True False

Don't

Know

10. A part of the anger cycle involves punishing

True False

Don't yourself by using substances.

Know 
Evaluation of a Correctional Substance Abuse Program 254

Appendix J

Denial Video Questionnaire 


\section{$\underline{\text { Denial }}$}

This questionnaire consists of 10 statements about denial. Some of the statements are true and others are false. If you think a statement is true, circle the word "True". If you think a statement is false, circle the word "False". If you do not know whether a statement is true or false, circle "Don't Know".

1. Someone who refuses to admit they have a substance abuse problem is in denial.

True False Don't

True False Don't

2. Denial is a defence mechanism.

3. Blaming is a form of denial.

True False Don't

4. Exploiting is a form of denial.

True False

Don't

Know

5. Intellectualizing is when someone can apply their knowledge of substance abuse to themselves.

6. Rationalizing is when someone blames another person for their substance abuse.

True False

Don't

Know

True False Don't

Know

True False Don't

Know substance use is not a problem and compares their use to someone who they think uses too much.

8. Reflection is a method for overcoming denial. True False Don't Know

9. Denial is progressive and gets stronger over time.

True False

Don't

Know

10. Diversion is a not form of denial.

True False

Don't

Know 
Evaluation of a Correctional Substance Abuse Program 256

Appendix K

My Father's Son Video Questionnaire 


\section{My Father's Son}

This questionnaire consists of 5 statements about the development of substance abuse problems. Some of the statements are true and others are false. If you think a statement is true, circle the word "True". If you think a statement is false, circle the word "False". If you do not know whether a statement is true or false, circle "Don't Know"

1. A person is more likely to develop a drinking problem if they drink hard alcohol as opposed True False Don't to beer.

2. Alcohol and drug abuse problems can be True False Don't passed on through families.

Know

3. Someone can be raised by an alcoholic parent and not become an alcoholic.

True False

Don't

4. In a family where a parent has a substance abuse problem, all of the children will True False Know eventually develop substance abuse problems.

5. When someone lives with a parent who has a True False Don't drug abuse problem, they will be able to recognize when their own use of drugs is a problem. 
Evaluation of a Correctional Substance Abuse Program 258

Appendix L

Preprogram Client Survey

Reproduced with permission of the copyright owner. Further reproduction prohibited without permission. 


\section{Preprogram Client Survey}

\section{Demographic Data (this page completed by counsellor)}

ID \#:

Date Completed Preprogram Client Survey:

Current Age: Learning Disabilities: Yes No

Education Level:

Less than grade 8

GED

Some high school

More than high school

Risk Level:

Need Level:

Type of Offence:

MDH Assessment of Substance Abuse Treatment Need:

Level on ADS:

Raw Score on ADS:

Level on DAST:

Raw Score on DAST:

Drug used most often:

Client participated in any previous substance abuse program(s)?: Yes

No

Participated in previous institutional treatment? Yes

No

If participated in previous substance abuse program(s) in an institution, what type of program(s)? [check off the program(s)]

$\begin{array}{llllll}\square & \text { AA } & & \text { OSAPP } & \square & \text { Drug \& Alcohol Education } \\ \square & \text { NA } & \square & \text { Counselling } & \square & \text { Therapeutic Community } \\ \square & \text { Self-help (other than AA/NA) } & \square & \text { Drug Free Unit } & & \text { (such as Riverhill, Echo, Stop) } \\ \square & \text { Other } & & & \end{array}$

Participated in previous community treatment? Yes

No

If participated in previous substance abuse program(s) in the community, what type of program(s)? [check off the program(s)]
( $\mathrm{AA}$
$\square$ NA
[ CHOICES
Self-help (other than AANA)
Counselling
$\square$ Other
Drug \& Alcohol Education
Therapeutic Community
Relapse Prevention
(other than CHOICES)

If participated in previous substance abuse program(s), how many programs have been completed in the institution? [total number completed]

If participated in previous substance abuse program(s), how many programs have been completed in the community? [total number completed] 
The first part of this survey contains several questions about your participation in the Maison Decision House (MDH) Substance Abuse Program. The second part of the survey asks about your physical and emotional health, family and friends as well as leisure activities.

To answer most of the questions on this survey you simply circle the numbered response on the corresponding scale that best describes you. For a couple of the questions you will need to write your response in the response area provided.

Completing this survey is very helpful to the MDH program because it allows us to make sure the program is meeting your needs and helping you to deal with your substance abuse. Your feedback is important and we would like to thank you for your time and cooperation.

\section{PART 1 - Participation in the MDH Substance Abuse Treatment Program}

1. How much do you personally want to take part in the MDH Substance Abuse Treatment Program?

Not at All

1
A little

2

3

4

A lot

2. How much do you personally want to stop abusing substances?

Not at All

1
A little

3
A lot

2
4 5

3. How much would you want to be involved in a substance abuse treatment program if the decision was completely your own?

Not at All

1
A little

3

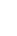

A lot

2
4 5

4. How much do you want to abstain from substances?

Not at All

1
A little

3 
5. How much knowledge do you have in each of the following areas:

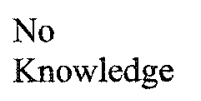

a) being assertive (speaking your mind)

b) dealing with your anger

c) dealing with stress in your life

d) solving problems in your life

e) developing your own relapse prevention plan

f) identifying your own highrisk situations

g) using relapse prevention techniques

\section{Some} Knowledge
A lot of Knowledge

1

2

3

4

5

5

5

5

5

1

2

3

4

5

1

2

3

4

5

1

2

3

4

5

6. How much ability do you have with each of the following areas:

\section{No}

Ability
Some

Ability

\section{A lot of}

Ability

a) being assertive (speaking your mind)

1

2

3

4

5

b) dealing with your anger

2

3

4

5

c) dealing with stress in your life

2

3

4

5

d) solving problems in your life

1

2

3

4

5

e) developing your own

2

3

4

5

relapse prevention plan

f) identifying your own high-

1

2

3

4

5 risk situations

g) using relapse prevention

1

2

4 5 
7. How confident are you that you would not use substances if you had the chance right now?

Not at all

Somewhat

Very

Confident

Confident

Confident

1

2

3

4

5

PART 2 - Your Physical and Emotional Health, Family and Friends, Leisure Activities

8. How often do you feel tense?

Never 1
Sometimes

3

All the Time

2

4

5

9. How often do you feel depressed?

Never 1
Sometimes

3

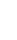

4

All the Time 2 4 5

10. How often do you feel tired?

Never 1
Sometimes

3
All the Time

4 5

11. How much sleep do you get a night? (hours)

12. How often do you feel low self-esteem?

Never 1
Sometimes

3
All the Time

4 5

13. How often do you want to use substance(s)?

Never

1
Sometimes

3
All the Time

4 
14. How often are you in contact with your family? (For example, your spouse or partner, children, parents and/or grandparents).

Never Sometimes Frequently

$\begin{array}{lllll}1 & 3 & 4 & 5\end{array}$

15. How satisfied are you with your level of contact with your family? (For example, your spouse or partner, children, parents and/or grandparents).

Not

Satisfied

Very

Satisfied

Satisfied

1

2

3

4

5

16. How often are you in contact with your friends?

Never

Sometimes

Frequently

1

2

3

4

5

17. How satisfied are you with your level of contact with your friends?

Not

Satisfied

Very

Satisfied

Satisfied

1

2

3

4

5

18. How often do you get involved with leisure activities? (For example, doing sports, going to the movies, reading, writing and/or surfing the web).

Never

Sometimes

Frequently

1

2

3

4

5

19. How satisfied are you with your level of involvement with leisure activities?

Not

Satisfied

Very

Satisfied

Satisfied 
Evaluation of a Correctional Substance Abuse Program 264

Appendix M

Postprogram Client Survey 
Evaluation of a Correctional Substance Abuse Program 265

\section{Postprogram Client Survey}

For office use

Name:

FPS \#:

Date Completed Postprogram Client Survey:

Date Started the MDH Program:

Completed MDH Program? Yes No 
The first part of this survey contains several questions about your participation in the Maison Decision House (MDH) Substance Abuse Treatment Program. The second part of the survey asks about your physical and emotional health, family and friends, leisure activities as well as employment. To answer most of the questions on this survey you simply circle the numbered response on the corresponding scale that best describes you. For a couple of the questions you will need to write your response in the response area provided.

Completing this survey is very helpful to the MDH program because it allows us to make sure the program is meeting your needs and helping you to deal with your substance abuse. Your feedback is important and we would like to thank you for your time and cooperation.

\section{PART 1 - Participation in the MDH Substance Abuse Treatment Program}

1. Overall, how satisfied are you with this substance abuse treatment program?

Not

Satisfied

Very

Satisfied

Satisfied

1

2

3

4

5

2. How satisfied are you with the four mandatory substance abuse videos you watched when you started the MDH program? (the videos were Denial, Shame and Addiction, My Father's Son and Unresolved Anger)

Not

Satisfied

Very

Satisfied

Satisfied

1

2

3

4

5

3. How satisfied are you with the material presented in the four mandatory videos you watched when you started the program? (the videos were Denial, Shame and Addiction, My Father's Son and Unresolved Anger)

Not

Satisfied

Satisfied

Very

Satisfied 
4. How much did your knowledge of substance abuse issues, such as denial, shame, addiction and anger increase after watching the substance abuse videos at the beginning of the program?

Did Not

Increased

Greatly

Increase

Somewhat

Increased
1
2
3
4
5

5. How satisfied are you with the number of individual counselling sessions you had?

Not

Satisfied

Very

Satisfied

Satisfied

1

2

3

4

5

6. How satisfied are you with the topics you and your counsellor talked about during your individual counselling sessions?

Not

Satisfied

Very

Satisfied

Satisfied

1

2

3

4

5

7. How satisfied are you with the ability of your counsellor to help you deal with the difficult areas in your life?

Not

Satisfied

Very

Satisfied

Satisfied

1

2

3

4

8. How much did your motivation to participate in the MDH Substance Abuse

Treatment program increase after your first counselling session?

Did Not

Increase

1
Increased

Somewhat

3
Greatly

Increased

4 5

9. How satisfied are you with the number of group sessions you attended?

Not

Satisfied
Satisfied

3
Very

Satisfied

4 
10. How satisfied are you with the material presented in the group sessions?

Not

Satisfied

Very

Satisfied

Satisfied
1
2
3
4
5

11. How satisfied are you with the way the instructors presented the material in the group sessions?

Not

Satisfied

Very

Satisfied

Satisfied

1

2

3

4

5

12. On each of the following issues, how much did your knowledge increase after completing the group sessions:

$\begin{array}{lcr}\text { Did not } & \text { Increased } & \begin{array}{r}\text { Greatly } \\ \text { Increased }\end{array}\end{array}$
a) being assertive (speaking your mind)
1
2
3
4
b) dealing with your anger
2
3
4
5
c) dealing with stress in your life

1
2
3
4
5
d) solving problems in your life
1
2
3
4
5
e) developing your own
relapse prevention plan

1
2
3
4
5
f) identifying your own high-
1
2
3
4
5 risk situations
g) using relapse prevention techniques


13. On each of the following issues, how much did your ability increase after completing the group sessions:

\section{Did not}

Increase

a) being assertive (speaking your mind)

b) dealing with your anger

c) dealing with stress in your life

d) solving problems in your life

e) developing your own relapse prevention plan

f) identifying your own highrisk situations

g) using relapse prevention techniques
Increased

Somewhat

2

2

2

2

1.2

2

2

2

3

3

3

3

3
4

5

5

14. How much did your motivation to take part in the MDH Substance Abuse Treatment Program increase while participating in the program?

Did Not

Increased

Increase

Somewhat
Greatly

Increased

1

2

3

4

5

15. How much has your motivation to stop abusing substances increased while participating in the program?

Did Not

Increase

1
Increased

Somewhat

3
Greatly

Increased

16. How much do you want to abstain from substances?

Not at All

1
A little

3
A lot 
17. How much knowledge do you have in each of the following areas:

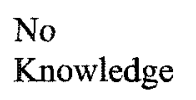

a) being assertive (speaking your mind)

b) dealing with your anger

c) dealing with stress in your life

d) solving problems in your life

e) developing your own

f) identifying your own highrisk situations

g) using relapse prevention techniques relapse prevention plan

\section{Some Knowledge}

3

3

3

3

3

4

4

18. How much ability do you have with each of the following areas:
5

A lot of Knowledge

5

5

5

5

5

5

\section{Some}

Ability
No
Ability

a) being assertive (speaking your mind)

b) dealing with your anger

c) dealing with stress in your life

d) solving problems in your life

e) developing your own relapse prevention plan

f) identifying your own highrisk situations

g) using relapse prevention techniques

$\begin{array}{lllll}1 & 2 & 3 & 4 & 5 \\ 1 & 2 & 3 & 4 & 5 \\ 1 & 2 & 3 & 4 & 5 \\ 1 & 2 & 3 & 4 & 5 \\ 1 & 2 & 3 & 4 & 5 \\ 1 & 2 & 3 & 4 & 5 \\ 1 & 2 & 3 & 4 & 5\end{array}$

A lot of

Ability

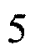


19. How confident are you that you would not use substances if you had the chance right now?

Not at all

Somewhat

Very

Confident

Confident

Confident

1

2

3

4

5

PART 2 - Your Physical and Emotional Health, Family and Friends, Leisure Activities

20. How often do you feel tense?

Never

1
Sometimes

3

2

21. How often do you feel depressed?

Never

Sometimes

2

3

4

1
22. How often do you feel tired?

Never

Sometimes

3

4 (hours)

23. How much sleep do you get a night?

1

2

All the Time

4

5

All the Time

24. How often do you feel low self-esteem?

Never 1

Sometimes

All the Time

3

4 5

25. How often do you want to use substance(s)?

Never

1
Sometimes

3
All the Time

4 
26. How often are you in contact with your family? (For example, your spouse or partner, children, parents and/or grandparents).

Never

Sometimes

Frequently

$\begin{array}{lllll}1 & 3 & 4 & 5\end{array}$

27. How satisfied are you with your level of contact with your family? (For example, your spouse or partner, children, parents and/or grandparents).

Not

Satisfied

Very

Satisfied

Satisfied

1

2

3

4

5

28. How often are you in contact with your friends?

Never

Sometimes

Frequently

1

2

3

4

5

29. How satisfied are you with your level of contact with your friends?

Not

Satisfied

Satisfied

Very

Satisfied

1

2

3

4

5

30. How often do you get involved with leisure activities? (For example, doing sports, going to the movies, reading, writing and/or surfing the web).

Never

Sometimes

Frequently

1

2

3

4

5

31. How satisfied are you with your level of involvement with leisure activities?

Not

Satisfied

Very

Satisfied

Satisfied 
Evaluation of a Correctional Substance Abuse Program 273

Appendix N

Balanced Inventory of Desirable Responding 


\section{$\underline{\text { BDR }}$}

Name: FPS\#:

Date Completed:

Using the scale below as a guide, write a number in the space on the left side of the statement to indicate how much you agree with it.

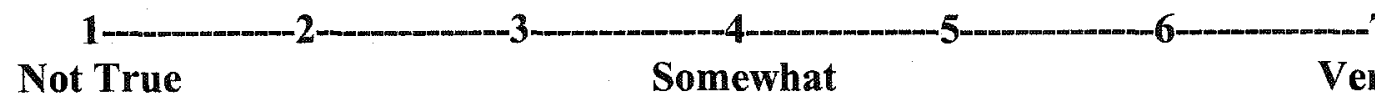

(For office use)

1. My first impressions of people usually turn out to be right.

2. It would be hard for me to break any of my bad habits.

3. I don't care to know what other people really think of me.

4. I have not always been honest with myself.

5. I always know why I like things.

6. When my emotions are aroused, it biases my thinking.

7. Once I've made up my mind, other people can seldom change my opinion.

8. I am not a safe driver when I exceed the speed limit.

9. I am fully in control of my own fate.

10. It's hard for me to shut off a disturbing thought.

11. I never regret my decisions.

12. I sometimes lose out on things because I can't make up my mind

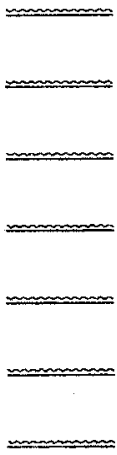
soon enough.

13. The reason I vote is because my vote can make a difference.

14. My parents were not always fair when they punished me.

15. I am a completely rational person.

16. I rarely appreciate criticism.

17. I am very confident in my judgements.

18. I have sometimes doubted my ability as a lover.

19. It's all right with me if some people happen to dislike me.

20. I don't always know the reason why I do the things I do.

TOTAL: 


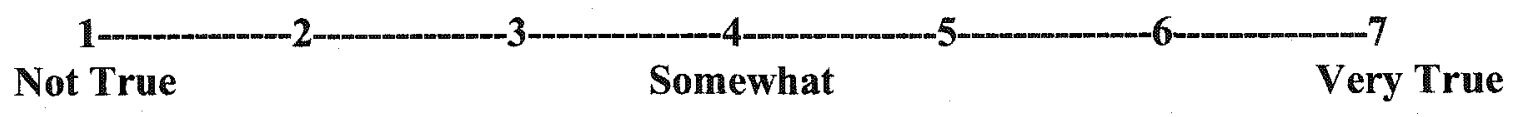

(For office use)

21. I sometimes tell lies if I have to.

22. I never cover up my mistakes.

23. There have been occasions when I have taken advantage of someone.

24. I never swear.

25. I sometimes try to get even rather than forgive and forget.

26. I always obey laws, even if I'm unlikely to ever get caught.

27. I have said something bad about a friend behind his or her back.

28. When I hear people talking privately, I avoid listening.

29. I have received too much change from a salesperson without telling him or her.

30. I always declare everything at customs.

31. When I was young sometimes I stole things.

32. I have never dropped litter on the street.

33. I sometimes drive faster than the speed limit.

34. I never read sexy books or magazines.

35. I have done things that I don't tell other people about.

36. I never take things that don't belong to me.

37. I have taken sick-leave from work or school even though I wasn't really sick.

38. I have never damaged a library book or store merchandise without reporting it.

39. I have some pretty awful habits.

40. I don't gossip about other people's business. 
Evaluation of a Correctional Substance Abuse Program 276

Appendix $\mathrm{O}$

Pre- and Postprogram Staff Survey 


\section{Pre- and Postprogram Staff Survey}

Name:

FPS\#:

\section{Preprogram Data:}

Date Completed Preprogram Survey:

1. In your professional opinion, how motivated is the offender to participate in the MDH substance abuse treatment program (at program intake)?

Not at all

Motivated
Somewhat

Motivated

2. In your professional opinion, what stage of change is the offender in (at program intake)? Please put a check mark in the corresponding box.

Precontemplation

Contemplation

Action

3. In your professional opinion, how confident is the client that he will not use substances if he had the chance right now (at program intake)?

Not at all

Confident

1
Somewhat

Confident

3
Very

Confident

4

5

\section{Postprogram Data:}

Date Completed Postprogram Survey:

4. In your professional opinion, how motivated is the offender to participate in the MDH substance abuse treatment program (at program departure)?

Not at all

Motivated

1
Somewhat

Motivated
Very

Motivated 
5. In your professional opinion, what stage of change is the offender in (at program departure)? Please put a check mark in the corresponding box.

Precontemplation

Contemplation

Action

6. In your professional opinion, how confident is the client that he will not use substances if he had the chance right now (at program intake)?

Not at all

Confident

1
Somewhat

Confident

3
Very

Confident

4

7. In your professional opinion, how much knowledge does the offender have in each of the following areas (at program departure):

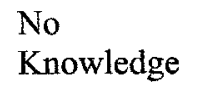

a) being assertive (speaking your mind)

b) dealing with your anger

c) dealing with stress in your life

d) solving problems in your life

e) developing your own relapse prevention plan

f) identifying your own highrisk situations

g) using relapse prevention techniques
Some

Knowledge

2

2

2

2

2

1

1

2

3

4

5

1

2

3

4

5 
8. In your professional opinion, how much ability does the offender have in each of the following areas (at program departure):

\section{No}

Ability

a) being assertive (speaking your mind)

b) dealing with your anger

c) dealing with stress in your life

d) solving problems in your life

e) developing your own relapse prevention plan

f) identifying your own highrisk situations

g) using relapse prevention techniques

\begin{abstract}
Some
\end{abstract}
Ability

1

1

1

1

1

1

1
2

2

2

2

2

2

2
3

3

3

3

3

3

3

4
5

A lot of

Ability

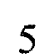

5

5

5

5

5

5


Evaluation of a Correctional Substance Abuse Program 280

Appendix $\mathbf{P}$

Key Informant Interview for Executive Director 


\section{Principles of Effective Correctional Treatment - Key Informant Interview}

\section{Risk Principle}

Treatment matched to offender's risk level (high need offender should receive intensive treatment)

- Does the program accept only those offender's with moderate to high risk, given that the MDH program is considered to be an intensive substance abuse treatment program?

\section{Need Principle}

Criminogenic need factors addressed through treatment

- Does the program address criminogenic need factors?

\section{Responsivity Principle}

General responsivity = program uses delivery methods consistent with offender learning style (i.e., cognitive-behavioural and social learning approaches)

- Is the program delivered using cognitive-behavioural and/or social learning approaches?

Specific responsivity $=$ offender characteristics matched to case worker/therapist characteristics

- Are the personal characteristics of an offender (such as, interpersonal sensitivity, interpersonal anxiety, verbal intelligence and cognitive maturity) matched to the program counsellor's characteristics?

\section{Multimodal Programming}

Program should incorporate a broad range of therapeutic modalities

- Does the program incorporate a range of therapeutic modalities?

\section{Community Programming}

Programs located in the community yield more positive effects than institutional programs

- Is the program based in the community? 


\section{Continuity of Care}

Aftercare should be provided to offenders once they have completed the formal treatment component

- Is aftercare provided once offenders graduate from the program?

\section{Length of Treatment}

It is recommended that correctional programs be about 100 hours in length and be delivered over a 3 to 6 month period

- Does the program consist of 100 hours?

- Are the program hours delivered over 3 to 6 months?

\section{Program Integrity}

Staff should have certain characteristics and qualifications

- Are program counsellors assessed on their interpersonal skills?

- Do program counsellors have undergraduate degrees or equivalent training on theories of criminal behaviour and prediction and treatment of criminal behaviour?

- Do staff receive 3 to 6 months of formal and on-the-job training?

Quality assurance - staff should be assessed periodically on quality of service delivery

- Are staff periodically assessed on the quality of service delivery?

Program monitoring - there should be continual assessment of adequate resources for the program and integration of ongoing program evaluation to facilitate the fulfilment of program goals

- Is the adequacy of the program's resources periodically assessed?

- Is program evaluation incorporated into the program? 
Evaluation of a Correctional Substance Abuse Program 283

Appendix Q

Key Informant Interview for Counsellors 


\section{Interview with Counsellors on Reading and Comprehension of Questionnaires}

1. In general, what do you think of the questionnaires?

2. Are there any questionnaires that cause clients more difficulty than others?

3. In your opinion, do clients read all of the questions on the questionnaires? If not, how often does this occur?

4. In your opinion, do clients understand the questions in questionnaires? If not, how often does this occur?

5. If a client cannot read, what is done to assist him? How often does this happen?

6. If a client does not understand a question, what is done to assist him? How often does this happen? 
Evaluation of a Correctional Substance Abuse Program 285

Appendix R

Consent Form 


\section{Informed Consent Form}

The purpose of an informed consent is to make sure you understand the purpose of the evaluation and how you will be involved. The informed consent provides enough information so you can decide if you want to participate in the evaluation.

Research title: Second-generation evaluation of the Maison Decision House correctional community substance abuse treatment program.

Research Personnel: The following people are involved in this evaluation and may be contacted at any time: Chantal Langevin (Main Researcher, Dept. of Psychology, Carleton University), Dr. Shelley Borys (Faculty Sponsor, 613-235-7215) and Dr. Robert Hoge (Faculty Sponsor, 613-5205773). If you have any ethical concerns about this evaluation, please contact Dr. M. Gick (Chair, Carleton University Research Ethics Committee for Psychological Research, 520-2600, ext. 2664) or Dr. K. Matheson (Chair, Dept. of Psychology, 520-2600, ext. 2648).

Purpose and requirements: The purpose of this evaluation is to assess the success of the Maison Decision House (MDH) substance abuse treatment program. Information collected for this evaluation will assist in the determination of whether this program is meeting the needs of program participants and how the program could be improved. If you participate, you will complete several questionnaires over the course of your participation in the program, in addition to questionnaires already administered by MDH. The questionnaires completed for this evaluation examine your knowledge and ability with various prosocial and relapse prevention skills, confidence and motivation. In addition, these questionnaires provide information on how you feel about your own knowledge and ability as well as your satisfaction with the MDH program. Some of the questions on the questionnaires ask about sensitive topics, such as your feelings about your substance abuse. Completing these questionnaires will be done at various times while you reside at $\mathrm{MDH}$, with completion times ranging between 10 and 45 minutes. Your total time commitment will be approximately 2 hours and 30 minutes. There are no known risks to program participants who participate in the data collection process of this program evaluation.

Right to withdraw: There is no reward for participating, and no penalty for not participating in the evaluation. You may withdraw at any time, which means you can refuse to participate or answer any of the questions on the additional questionnaires.

Anonymity/Confidentiality: Information gathered will be kept confidential. All information will be coded so that your name is not associated with the data. The coded data will be made available only to the researchers involved in this project. Individual program participants will not be identified in any reports on this evaluation.

Signatures: I have read the above description of the evaluation and I give my permission to the researcher to make use of the information gathered in the evaluation.

Participant Full Name (Print):

Participant Signature:

Date:

Researcher Name:

Chantal Langevin

Researcher Signature: 
Evaluation of a Correctional Substance Abuse Program 287

Appendix S

Debriefing Form 


\section{Debriefing Form}

Thank you for your participation in this evaluation. The purpose of this evaluation is to assess the success of the Maison Decision House (MDH) substance abuse treatment program. Information collected for this evaluation will assist in the determination of whether this program is meeting the needs of program participants and how the program could be improved.

The pre-program client survey you completed at program intake along with other MDH assessment instruments are being used to examine your knowledge and ability in areas, such as prosocial and relapse prevention skills as well as your interest in participating in the MDH program. The pre- and post-video questionnaires you completed before and after watching the mandatory educational videos allow for the examination of any changes in your knowledge of specific issues related to substance abuse. The purpose for re-administering the Readiness to Change Questionnaire after your first counselling session is to find out whether involvement in this activity increased your motivation to change your substance-abusing behaviour. Finally, you were asked to complete the post-program client survey at the end of your participation in the $\mathrm{MDH}$ program as well as other post-program assessment instruments, so we could examine any changes in knowledge and skill development over the course of the program as well as provide us with your personal thoughts on various components of the $\mathrm{MDH}$ program.

This evaluation is being carried out by Chantal Langevin, as part of a requirement for her Doctoral thesis at Carleton University.

If you have any questions or comments about this evaluation, you can contact Chantal Langevin (Main Researcher, Dept. of Psychology, Carleton University), Dr. Shelley Borys (Faculty Advisor, Carleton University) at (613) 235-7215 or Dr. Robert Hoge (Faculty Advisor, Carleton University) at (613) 520-5773. If you have any ethical concerns about this evaluation, please contact Dr. M. Gick (Chair, Carleton University Research Ethics Committee for Psychological Research, 520-2600, ext. 2664) or Dr. K. Matheson (Chair, Dept. of Psychology, Carleton University, 520-2600, ext. 2648).

If you have any concerns or distress after participating in this evaluation, you can either make an appointment to speak to your Maison Decision House counsellor or contact your counsellor by phone at (613-728-5013).

The time and effort you gave to this evaluation is much appreciated. 
Evaluation of a Correctional Substance Abuse Program 289

Appendix T

Data to Collect for the Methodologies 


\begin{tabular}{|c|c|c|c|}
\hline Impact Assessment & Client Survey & Staff Survey & $\begin{array}{l}\text { Key Informant } \\
\text { Interview }\end{array}$ \\
\hline $\begin{array}{l}\text { Increased } \\
\text { motivation (RCQ) } \\
\text { Increased SA } \\
\text { knowledge (video } \\
\text { questionnaires) } \\
\text { Increased } \\
\text { knowledge with } \\
\text { prosocial skills } \\
\text { (PSQ and ASQ) } \\
\text { Increased } \\
\text { confidence } \\
\text { (DTCQ) }\end{array}$ & $\begin{array}{l}\text { Increased } \\
\text { knowledge of } \\
\text { prosocial skills } \\
\text { (pre/post) } \\
\square \text { Increased } \\
\text { knowledge of RP } \\
\text { skills (pre/post) } \\
\text { Ability with } \\
\text { prosocial skills } \\
\text { (pre/post) } \\
\text { Ability with RP } \\
\text { skills (pre/post) } \\
\text { Increased } \\
\text { confidence } \\
\text { (pre/post) } \\
\square \text { Increased } \\
\text { motivation } \\
\text { following } \\
\text { counselling (post) } \\
\square \text { Increased } \\
\text { knowledge of SA } \\
\text { following } \\
\text { drug/alcohol } \\
\text { education (post) } \\
\text { Increased } \\
\text { knowledge of } \\
\text { prosocial and RP } \\
\text { skills following } \\
\text { CBA (post) } \\
\square \text { Increase ability } \\
\text { with prosocial and } \\
\text { RP skills following } \\
\text { CBA (post) }\end{array}$ & $\begin{array}{l}\text { Increased } \\
\text { motivation } \\
\text { (pre/post) } \\
\text { Increased } \\
\text { knowledge of } \\
\text { prosocial skills } \\
\text { (post) } \\
\text { Increased } \\
\text { knowledge of RP } \\
\text { skills (post) } \\
\text { Ability with } \\
\text { prosocial skills } \\
\text { (post) } \\
\text { Ability with RP } \\
\text { skills (post) }\end{array}$ & $\begin{array}{l}\text { Risk } \\
\square \text { Need } \\
\square \text { Responsivity } \\
\square \text { Multimodal } \\
\text { programming } \\
\square \text { Community } \\
\text { programming } \\
\square \text { Continuity of care } \\
\square \text { Treatment length } \\
\square \text { Treatment integrity }\end{array}$ \\
\hline
\end{tabular}

Note. $\mathrm{RP}=$ relapse prevention; $\mathrm{DTCQ}=$ Drug-Taking Confidence Questionnaire; $\mathrm{RCQ}-\mathrm{TV}=$ Readiness to Change Questionnaire - Treatment Version; PSQ = Problem-Solving Questionnaire; $\mathrm{ASQ}=$ Assertiveness Questionnaire; $\mathrm{SA}=$ substance abuse; $\mathrm{CBA}=$ cognitive-behavioural activity. 
Evaluation of a Correctional Substance Abuse Program 291

Appendix U

Additional Tables for Results Section 
Table U1

Internal Consistency and Split-Half Estimates for the Client and Program Staff Surveys and the Pre- and Postvideo Questionnaires

\begin{tabular}{lccc}
\hline & & Cronbach's & $\begin{array}{c}\text { Guttman } \\
\text { split-half }\end{array}$ \\
Measure & 69 & $\alpha 2$ & $r$ \\
\hline & 22 & .92 & .88 \\
Preprogram client survey & 79 & .98 & .93 \\
Postprogram client survey & 77 & .57 & .96 \\
Preprogram staff survey & 34 & .96 & .71 \\
Postprogram staff survey & 27 & .67 & .87 \\
Prevideo total score & .85 & \\
Postvideo total score & & & \\
&
\end{tabular}


Table U2

Factor Loadings, Communalities, and Percentage of Variance for Principal Components

Analysis Using Varimax Rotation on the Pre-and Postprogram Client Survey and the

Postprogram Staff Survey for Knowledge of Relapse Prevention Skills

Item

Preprogram client survey ${ }^{\mathrm{a}}$

Knowledge of developing a relapse prevention plan

Knowledge of identifying high-risk situations

Knowledge of relapse prevention techniques

Postprogram client survey ${ }^{\mathrm{b}}$

Knowledge of developing a relapse prevention plan

Knowledge of identifying high-risk situations

Knowledge of relapse prevention techniques

Postprogram staff survey ${ }^{\mathrm{c}}$

Knowledge of developing a relapse prevention plan

Knowledge of identifying high-risk situations

Knowledge of relapse prevention techniques

$F_{1}$

$F_{1}$

.89 .79

.92

.84

.90

.81

.94

.87

.95

.91

.97

.94

.95

.90

.93

.87

.93

${ }^{a}$ Percentage of variance $=81.2 .{ }^{b}$ Percentage of variance $=90.6 .{ }^{c}$ Percentage of variance $=$ 87.7. 
Table U3

Factor Loadings, Communalities, and Percentage of Variance for Principal Components

Analysis Using Varimax Rotation on the Pre-and Postprogram Client Survey and the Postprogram Staff Survey for Ability of Relapse Prevention Skills

\begin{tabular}{llll}
\hline Item & $F_{1}$ & $h^{2}$ \\
\hline
\end{tabular}

Preprogram client survey ${ }^{a}$

Ability with developing a relapse prevention plan

.93 .86

Ability with identifying high-risk situations

.92

.85

Ability with relapse prevention techniques

.88

.78

Postprogram client survey ${ }^{b}$

Ability with developing a relapse prevention plan

.94

.92

.91

.94

.88

Ability with developing a relapse prevention plan

.91

.83

Ability with relapse prevention techniques

${ }^{\mathrm{a}}$ Percentage of variance $=83.0 .{ }^{\mathrm{b}}$ Percentage of variance $=85.0 .{ }^{\mathrm{c}}$ Percentage of variance $=$ 82.8 . 
Table U4

Correlation Matrix for the Posttest Measures of the DTCQ $(n=61)$

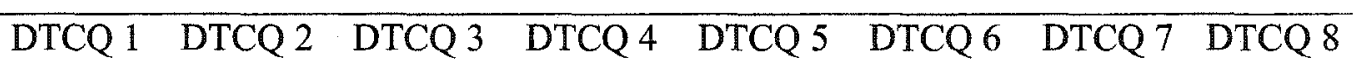

\begin{tabular}{|c|c|c|c|c|c|c|c|c|}
\hline DTCQ 1 & -- & & & & & & & \\
\hline DTCQ 2 & .76 & - & & & & & & \\
\hline DTCQ 3 & .94 & .78 & -- & & & & & \\
\hline DTCQ 4 & .80 & .84 & .71 & -- & & & & \\
\hline DTCQ 5 & .77 & .94 & .74 & .93 & -- & & & \\
\hline DTCQ 6 & .91 & .66 & .86 & .71 & .63 & -- & & \\
\hline DTCQ 7 & .90 & .67 & .80 & .80 & .69 & .91 & -- & \\
\hline DTCQ 8 & .83 & .54 & .72 & .64 & .54 & .88 & .83 & - \\
\hline
\end{tabular}

Note. All correlations were significant at $\mathrm{p}<.001$. 
Evaluation of a Correctional Substance Abuse Program 296

Table U5

Factor Loadings, Communalities, and Percentage of Variance for Principal Components

Analysis Using Varimax Rotation on the Postprogram Client Survey Items Measuring

Knowledge and Ability Following the Cognitive-Behavioural Activity

Item $\quad F_{1} \quad F_{2} \quad h^{2}$

Postprogram client survey

Ability with developing a relapse prevention plan

.88

Knowledge of identifying high-risk situations

.87

Knowledge of relapse prevention techniques

.87

.93

Ability with identifying high-risk situations

.86

Ability with relapse prevention techniques

.84

Knowledge of developing a relapse prevention plan

.82

Knowledge of assertiveness $.90 \quad .89$

Ability with assertiveness $.88 \quad .86$

Ability with problem-solving $.77 \quad .82$

Knowledge of problem-solving $.75 \quad .85$

Knowledge of problem-solving $.75 \quad .85$

Knowledge of anger $.71 \quad .69$

Ability with anger

Note. Percentage of variance for factor $1=46.4$; Percentage of variance for factor $2=$ 37.6 . 
Table U6

Checklist Matrix Analysis of the Principles of Effective Correctional Treatment

\begin{tabular}{|c|c|c|c|c|}
\hline \multirow[t]{2}{*}{ Principle } & \multicolumn{3}{|c|}{ Principle Incorporated } & \multirow[t]{2}{*}{ Notes } \\
\hline & Fully & Partially & $\begin{array}{c}\text { Not } \\
\text { Incorporated }\end{array}$ & \\
\hline 1. Risk & & $\checkmark$ & & $\begin{array}{l}\text { Sometimes accept } \\
\text { low risk b/c offender } \\
\text { assessed by MDH as } \\
\text { having high need for } \\
\text { substance abuse } \\
\text { treatment }\end{array}$ \\
\hline 2. Need & $\sqrt{ }$ & & & \\
\hline \multicolumn{5}{|l|}{ 3. Responsivity } \\
\hline \multirow{2}{*}{$\begin{array}{l}\text { a. General } \\
\text { b. Specific }\end{array}$} & $\checkmark$ & & & \\
\hline & & $\checkmark$ & & $\begin{array}{l}\text { Need to take into } \\
\text { account security and } \\
\text { caseloads }\end{array}$ \\
\hline 4. Multimodal Programming & $\checkmark$ & & & \\
\hline 5. Community Programming & $\checkmark$ & & & \\
\hline 6. Continuity of Care & $\checkmark$ & & & \\
\hline \multicolumn{5}{|l|}{ 7. Length of Program } \\
\hline \multirow{2}{*}{$\begin{array}{l}\text { a. } 100 \text { hours } \\
\text { b. Delivered over 3-9 months }\end{array}$} & $\sqrt{ }$ & & & \\
\hline & & & $\checkmark$ & $\begin{array}{l}\text { Program only funded } \\
\text { for } 8 \text {-week program }\end{array}$ \\
\hline \multicolumn{5}{|l|}{ 8. Program Integrity } \\
\hline \multicolumn{5}{|l|}{ a. Staff Characteristics \& Qualifications } \\
\hline \multirow{3}{*}{$\begin{array}{l}\text { i. Interpersonal skills assessed } \\
\text { ii. Undergraduate degree or } \\
\text { equivalent training } \\
\text { iii. 3-6 months of formal and on-job } \\
\text { training }\end{array}$} & $\checkmark$ & & & \\
\hline & $\checkmark$ & & & \\
\hline & & $\checkmark$ & & $\begin{array}{l}\text { Program staff gets } \\
\text { about } 1 \text { month of } \\
\text { formal and on-job } \\
\text { training }\end{array}$ \\
\hline \multicolumn{5}{|l|}{ b. Quality Assurance } \\
\hline $\begin{array}{l}\text { i. Periodic assessment of staff of } \\
\text { quality of program delivery }\end{array}$ & $\checkmark$ & & & \\
\hline \multicolumn{5}{|l|}{ c. Program Monitoring } \\
\hline \multirow{2}{*}{$\begin{array}{l}\text { i. Periodic assessment of financial } \\
\text { resources } \\
\text { ii. Evaluation incorporated into } \\
\text { program }\end{array}$} & $\checkmark$ & & & \\
\hline & $\checkmark$ & & & \\
\hline
\end{tabular}


Evaluation of a Correctional Substance Abuse Program 298

Appendix V

Profile Plots for Results Section 


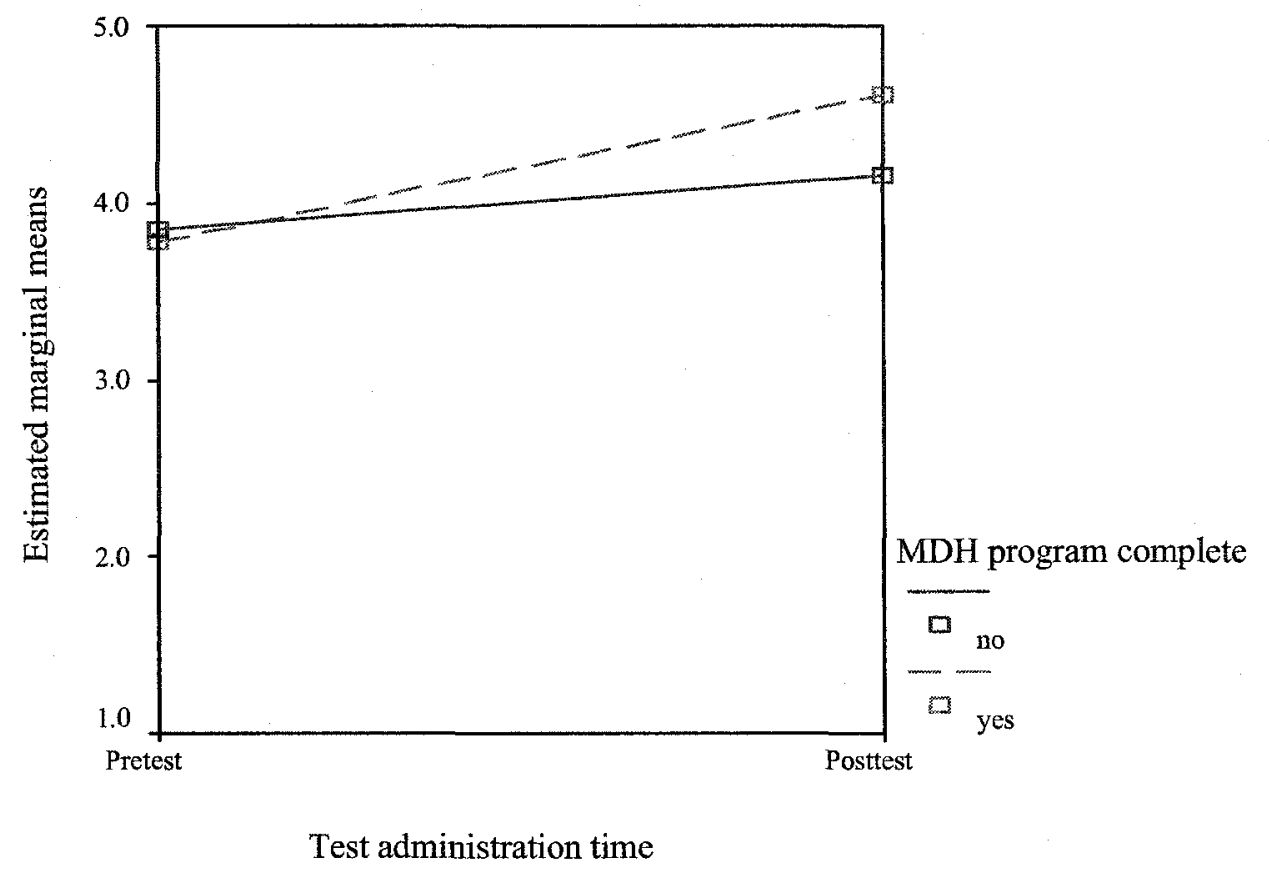

Figure V1. Mean scores on knowledge of assertiveness measured by the client survey across time for program completers and noncompleters.

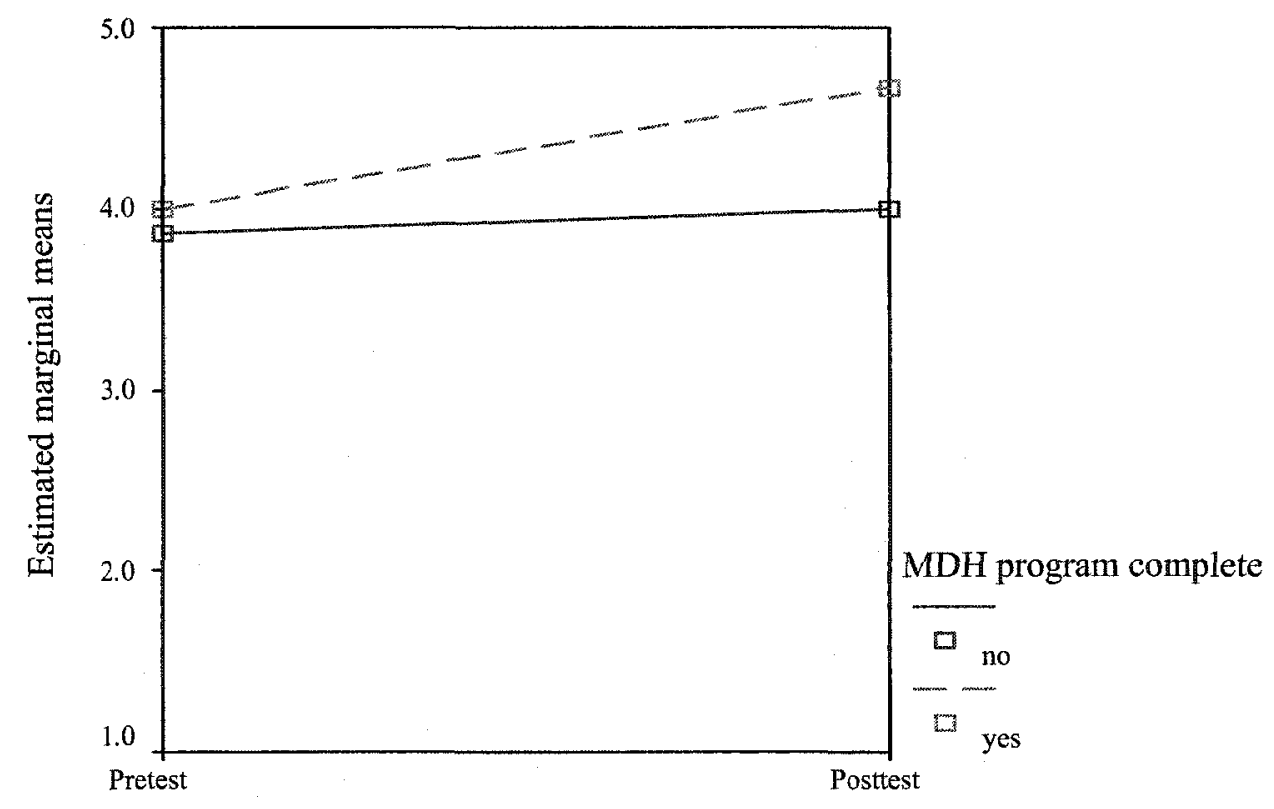

Test administration time

Figure V2. Mean scores on knowledge of anger measured by the client survey across time for program completers and noncompleters. 


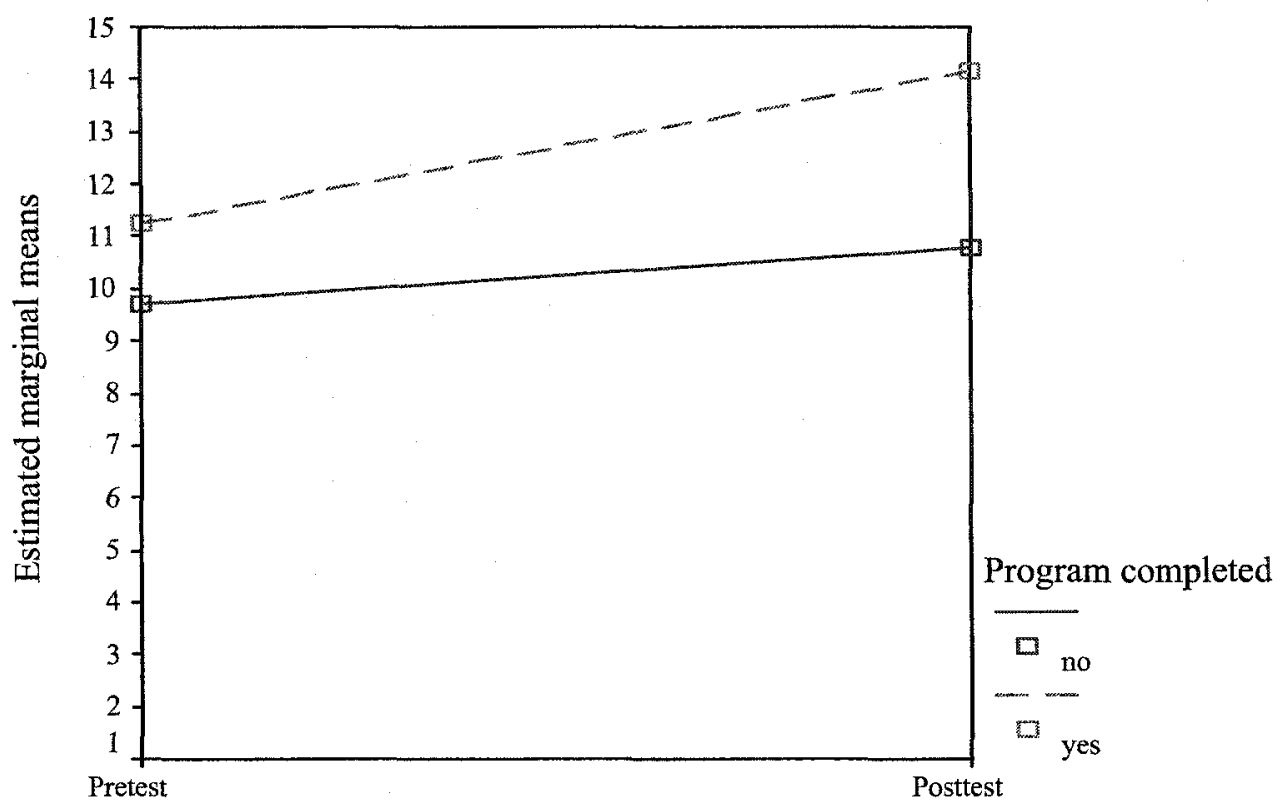

Test administration time

Figure V3. Mean scores on knowledge of relapse prevention skills measured by the client survey across time for program completers and noncompleters.

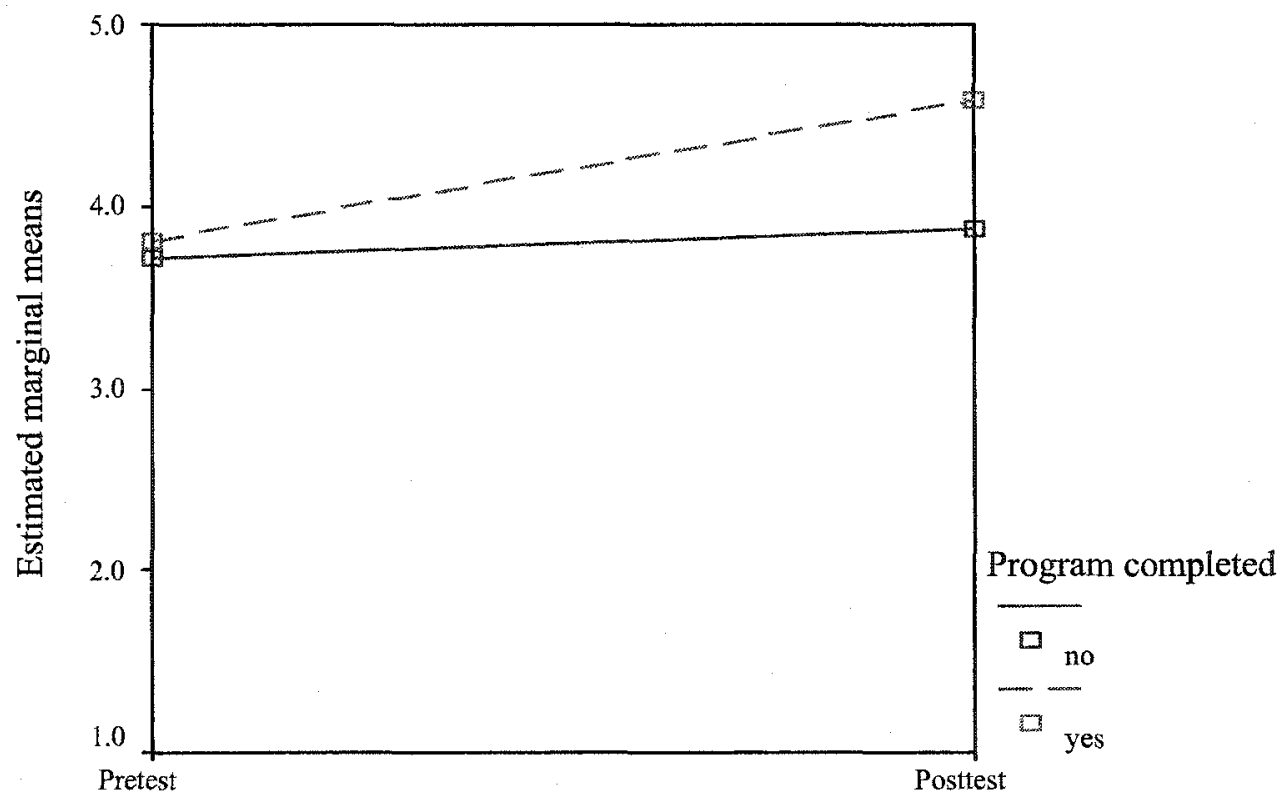

Test administration time

Figure V4. Mean scores on ability with assertiveness measured by the client survey across time for program completers and noncompleters. 


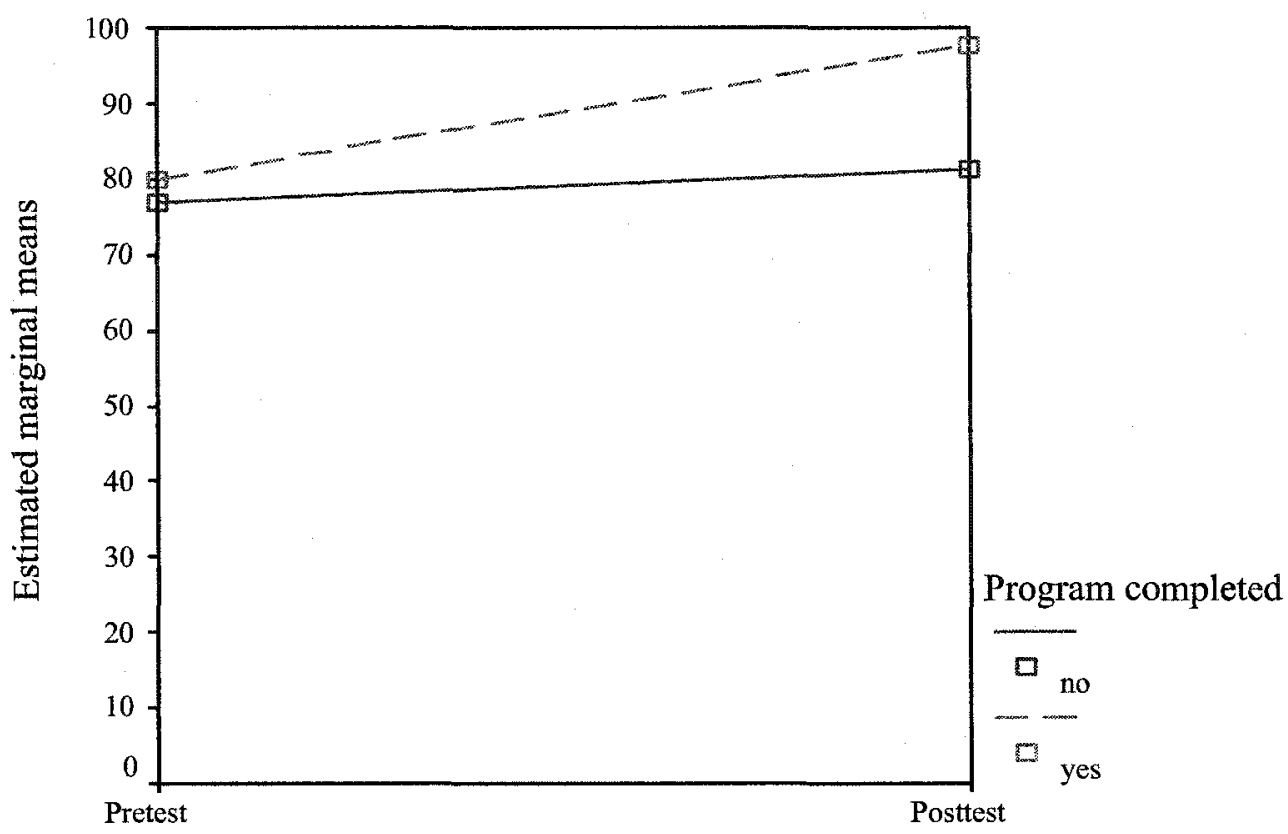

Test administration time

Figure V5. Mean scores on confidence to not use substances in positive situations measured by the DTCQ across time for program completers and noncompleters.

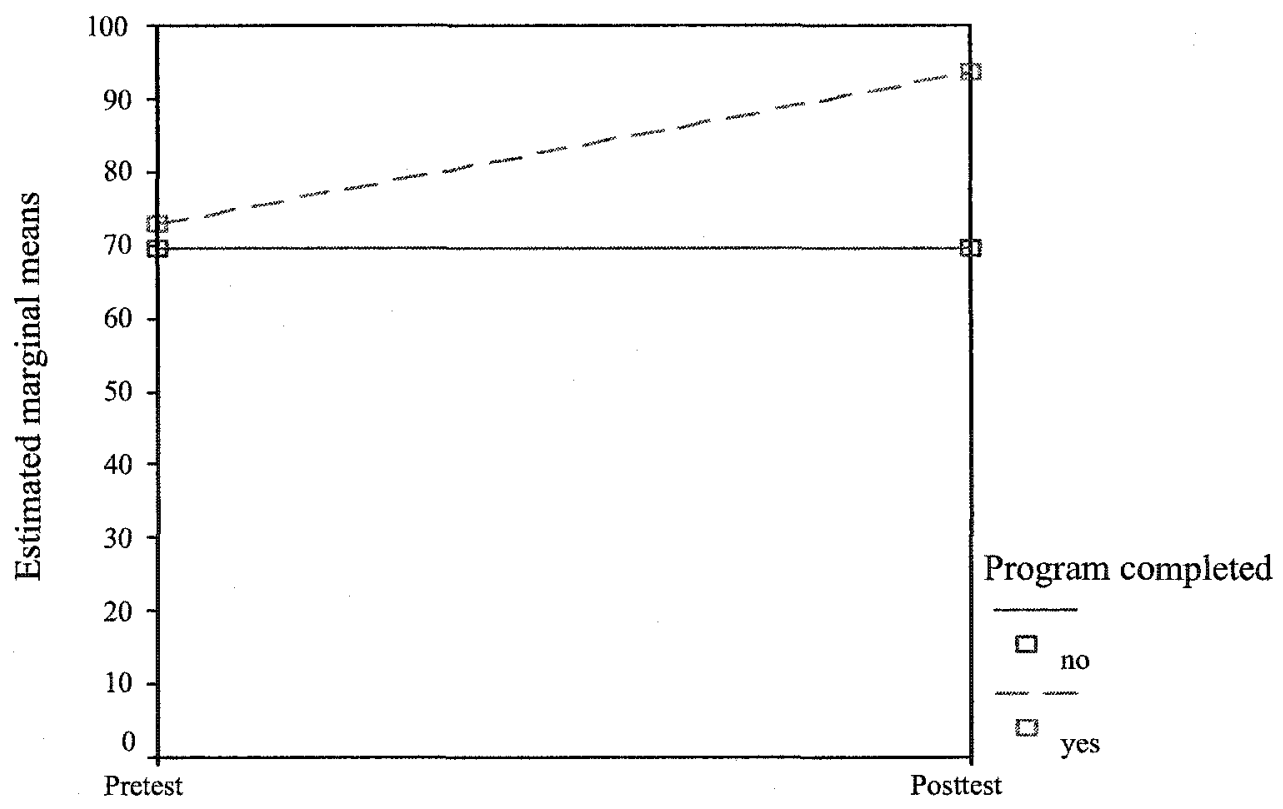

Test administration time

Figure V6. Mean scores on confidence to not use substances in temptation situations measured by the DTCQ across time for program completers and noncompleters. 


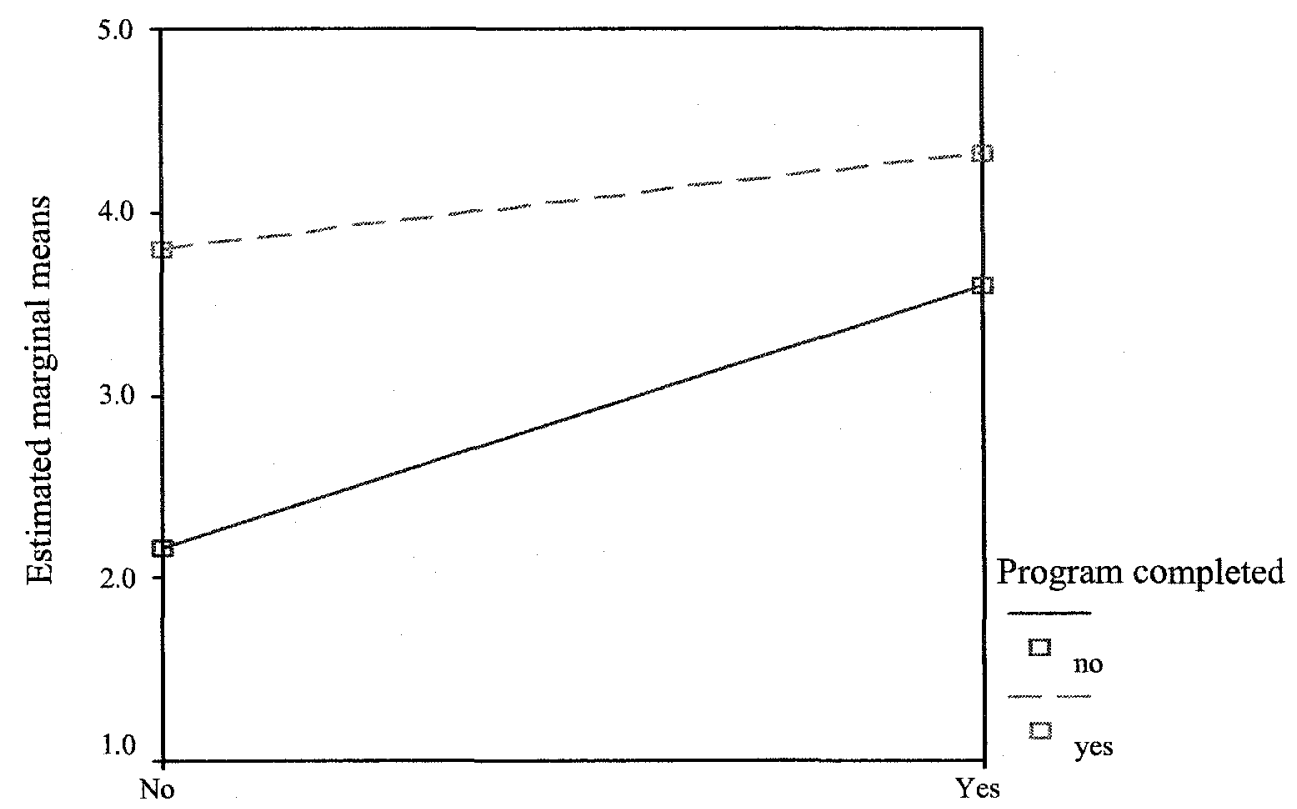

Revised initial counselling session

Figure V7. Mean scores on increased motivation following the counselling activity measure by the client survey for program completers and noncompleters. 